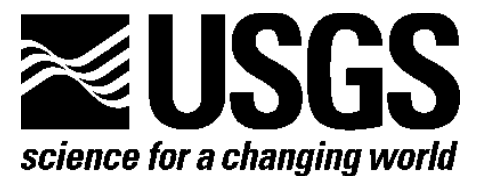

Prepared in cooperation with the U.S. Fish and Wildlife Service

\title{
Native Prairie Adaptive Management: A Multi Region Adaptive Approach to Invasive Plant Management on Fish and Wildlife Service Owned Native Prairies
}

By Jill J. Gannon, Terry L. Shaffer, and Clinton T. Moore

Open-File Report 2013-1279

U.S. Department of the Interior

U.S. Geological Survey 


\section{U.S. Department of the Interior \\ SALLY JEWELL, Secretary}

\section{U.S. Geological Survey \\ Suzette M. Kimball, Acting Director}

U.S. Geological Survey, Reston, Virginia: 2013

For more information on the USGS-the Federal source for science about the Earth,

its natural and living resources, natural hazards, and the environment-visit

http://www.usgs.gov or call 1-888-ASK-USGS

For an overview of USGS information products, including maps, imagery, and publications, visit $h$ ttp://www.usgs.gov/pubprod

To order this and other USGS information products, visit http://store.usgs.gov

Suggested citation:

Gannon, J.J., Shaffer, T.L., Moore, C.T., 2013, Native Prairie Adaptive Management: A Multi Region Adaptive Approach to Invasive Plant Management on Fish and Wildlife Service Owned Native Prairies: U.S. Geological Survey Open File Report 2013-1279, 184 p. with appendixes, http://dx.doi.org/10.3133/ofr20131279.

Any use of trade, firm, or product names is for descriptive purposes only and does not imply endorsement by the U.S. Government.

Although this information product, for the most part, is in the public domain, it also may contain copyrighted materials as noted in the text. Permission to reproduce copyrighted items must be secured from the copyright owner. 


\section{Contents}

Executive Summary

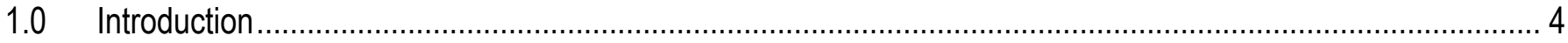

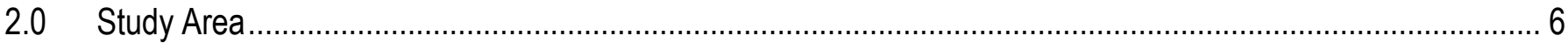

3.0 Adaptive Management Decision Support Framework ……..................................................................... 9

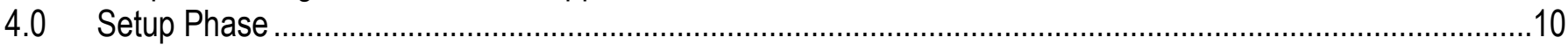

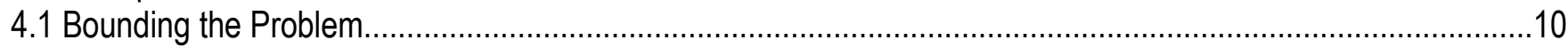

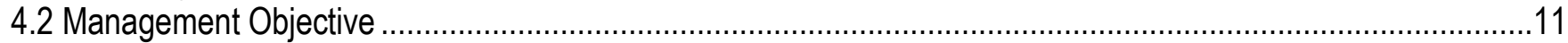

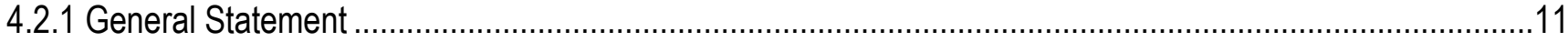

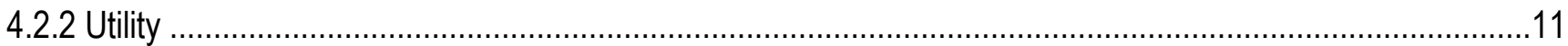

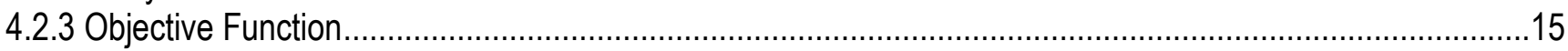

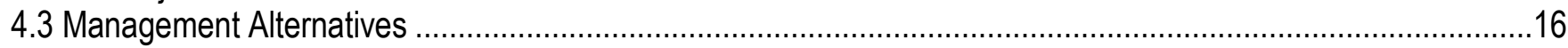

4.3.1 Provisional Prototype: Mixed-Grass and Tallgrass Alternative Management Actions.................................16

4.3.2 Final Prototype: Alternative Management Actions by Grass Type .........................................................16

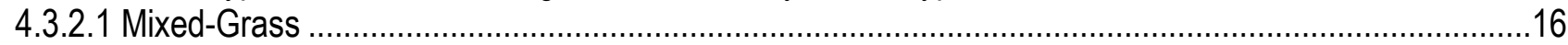

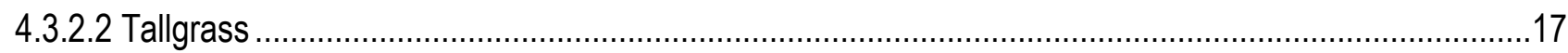

4.3.2.2.1 Sampling and Modeling Approaches for Making Post Hoc Determination of Tallgrass Management

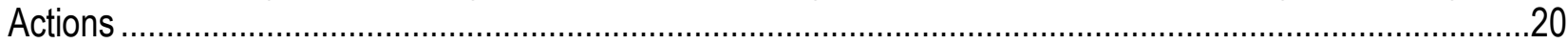

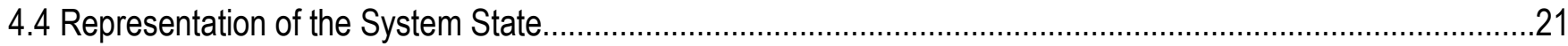

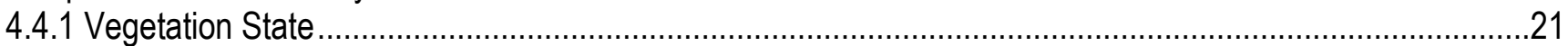

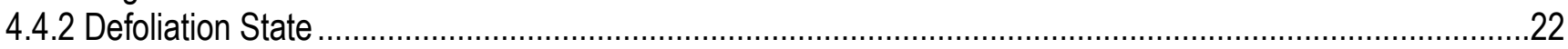

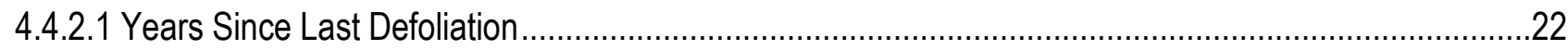

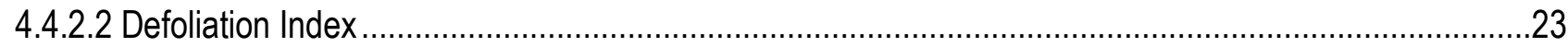

4.4.2.3 Combination of Classified Years Since Defoliation and Defoliation Index Level ..................................29

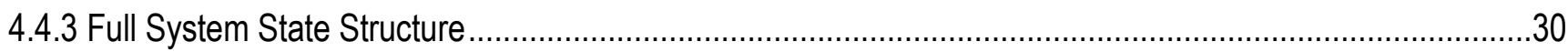

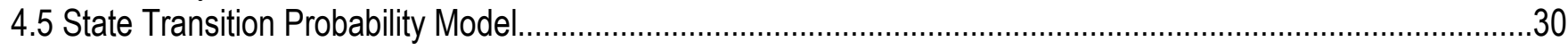

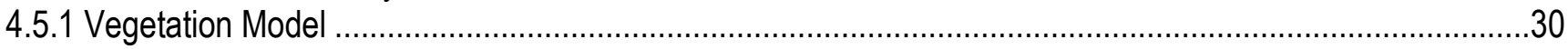

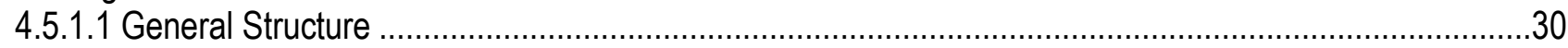

4.5.1.2 Model Parameterization .....................................................................................................3

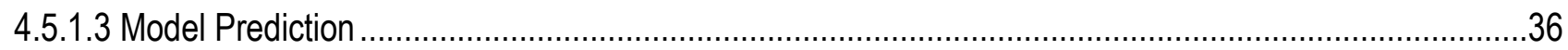

4.5.2 Defoliation Model................................................................................................................

4.5.3 Synthesis of the Vegetation and Defoliation Models.......................................................................38

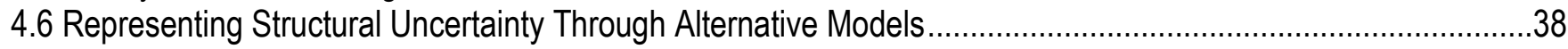

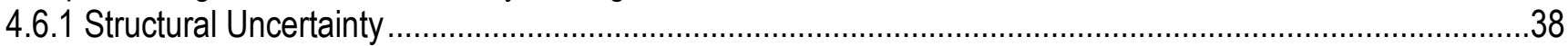

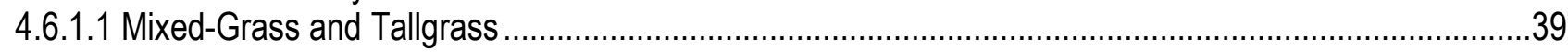

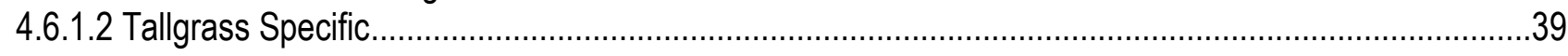

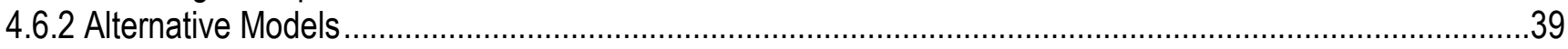

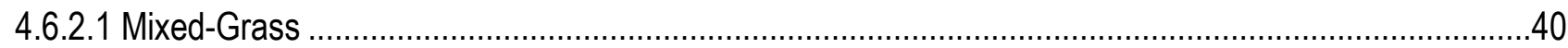

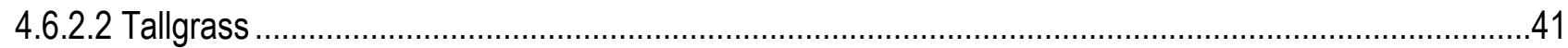

4.6.3 Parameterization of Alternative Models .......................................................................................4

4.6.4 Model Predictions_Implications of Alternative Models .....................................................................4

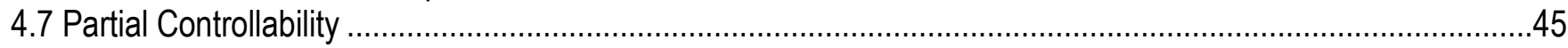

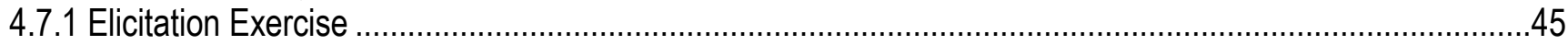

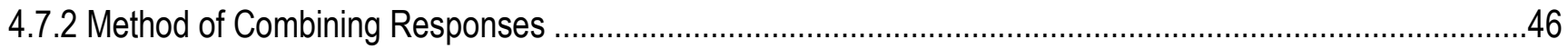

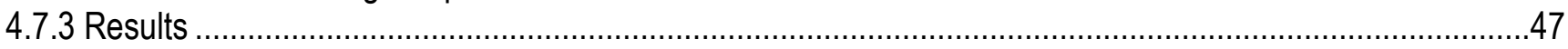

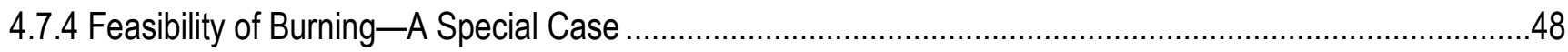

4.7.5 Management Restrictions.....................................................................................................

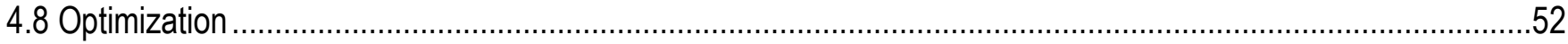




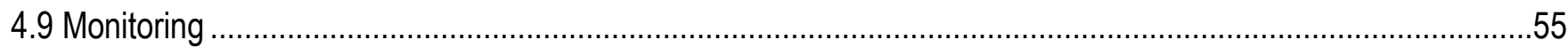

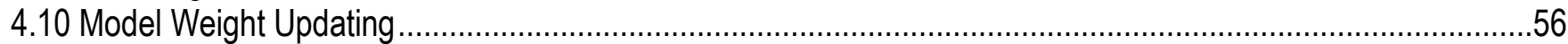

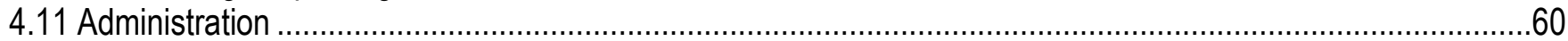

4.12 Database

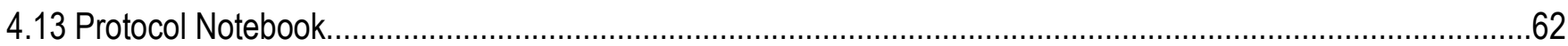

5.0 Iterative Phase

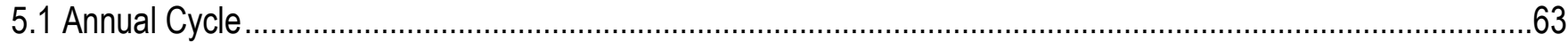

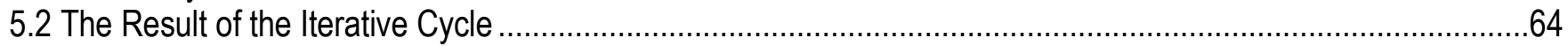

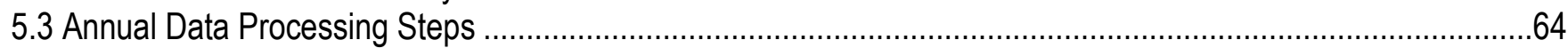

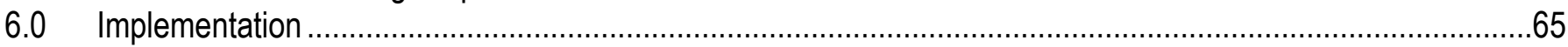

6.1 Summary of 2008-09

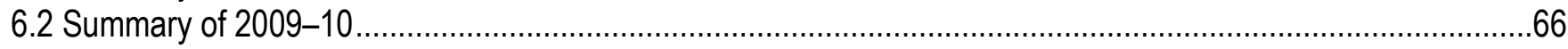

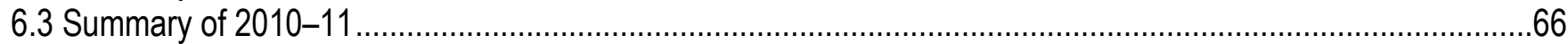

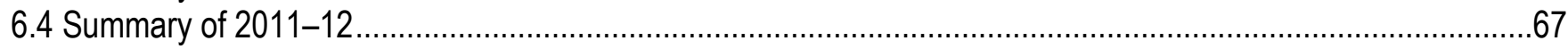

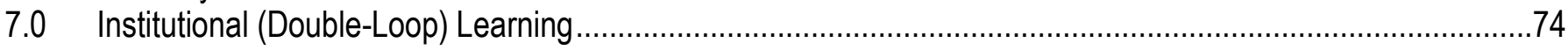

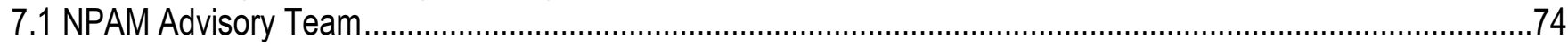

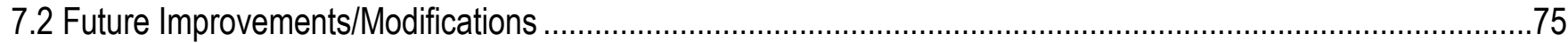

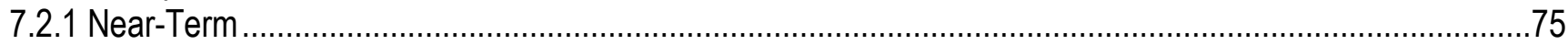

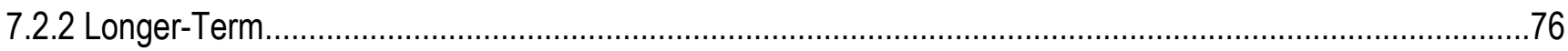

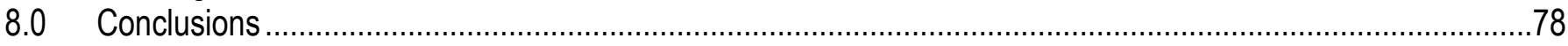

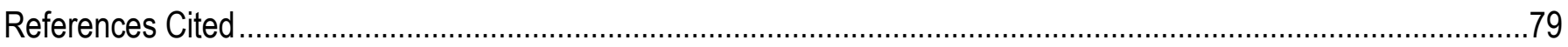

Appendixes

\section{Figures}

Figure 1. The Native Prairie Adaptive Management area of focus is native sod, on U.S. Fish and Wildlife Service (FWS)-owned lands, across the Prairie Pothole Region (PPR), within FWS regions 3 and $6 \ldots \ldots \ldots \ldots \ldots \ldots . . .7$

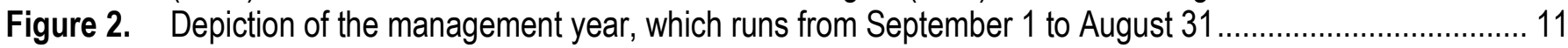

Figure 3. Location of the station-level sites monitored for phenology during 2011 and the tallgrass management units enrolled in the Native Prairie Adaptive Management initiative in 2011 ....................................... 21

Figure 4. Declining S-shaped logistic curve used to determine the weight for calculating the defoliation index...... 24

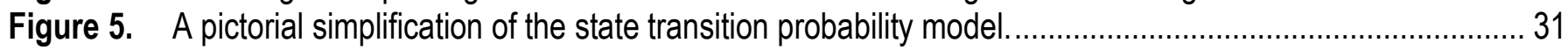

Figure 6. A single $16 \times 16$ matrix of the state transition probability model ....................................................... 32

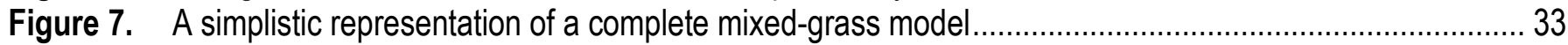

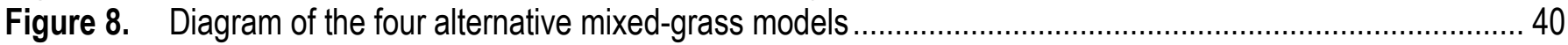

Figure 9. Diagram of the six alternative tallgrass models ............................................................................. 42

Figure 10. Hierarchical Dirichlet-multinomial model for computing posterior distribution .........................................58

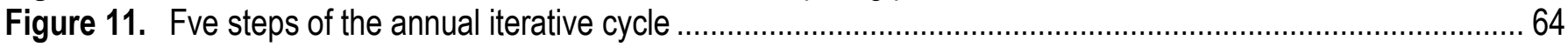

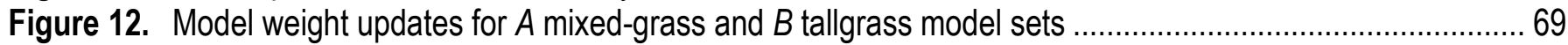

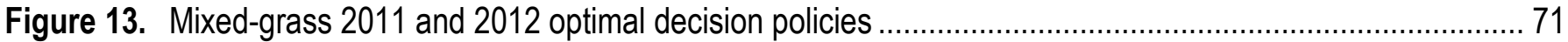

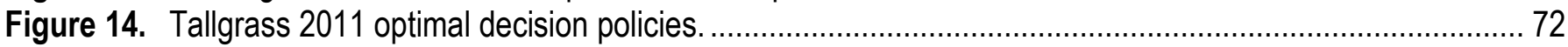

Figure 15. Tallgrass 2012 optimal decision policies...................................................................................... 73 


\section{Tables}

Table 1. The 19 U.S. Fish and Wildlife Service (FWS) Native Prairie Adaptive Management (NPAM) cooperators, number of management units each cooperator contributed to NPAM, and the grass type, state, and FWS administrative region of the unit.

Table 2. Utility for $A$ mixed-grass and $B$ tallgrass prairie management units ............................................ 12

Table 3. Vegetation states recognized in Native Prairie Adaptive Management............................................. 22

Table 4. Years since last defoliation over a seven-year window of past management history.......................... 23

Table 5. The weights used in calculating the defoliation index .................................................................. 24

Table 6. Defoliation indices for the 128 potential management histories over the seven-year window. …...... 25

Table 7. Distribution of the 128 past management history scenarios among the possible combinations of 'years since last defoliation' by 'number of defoliations' ............................................................... 28

Table 8. Probabilities of transitioning among the three defoliation levels in a one-year time step, given the choice of either rest or some form of defoliation as the management action within that time step..... 29

Table 9. A combination of the years since last defoliation and the defoliation index level results in seven discrete defoliation states.

Table 10. Skeleton of the format used to elicit the information necessary to parameterize the mixed-grass

vegetation model. ..................................................................................................... 34

Table 11. Probabilities of transitioning among the seven defoliation states in a one-year time step, given the choice of either $A$ rest or $B$ defoliation as the management action within that time step.................... 37

Table 12. Blank $4 \times 4$ partial controllability matrix used by cooperators with mixed-grass units........................ 46

Table 13. Partial controllability matrices for $A$ mixed-grass and $B$ tallgrass units. ......................................... 47

Table 14. Partial controllability matrices for $A$ mixed-grass and $B$ tallgrass units when the unit has been defoliated in the previous management year........................................................................ 49

Table 15. Partial controllability matrices for $A$ mixed-grass and $B$ tallgrass units with 'No Graze' restrictions... 50 Table 16. Partial controllability matrices for $A$ mixed-grass and $B$ tallgrass units with 'No Burn' restrictions ..... 51 Table 17. Partial controllability matrices for $A$ mixed-grass and $B$ tallgrass units with 'No Graze' restrictions when the unit has been defoliated in the previous management year.......................................... 51

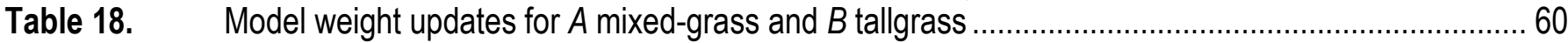

Table 19. Results of model weight updating cycles for the $A$ mixed-grass and $B$ tallgrass model set.............. 70

\section{Appendixes}

Appendix 1a. Description of exercise designed to elicit from Fish and Wildlife Service members of the Science Team information necessary to determine the vegetation state structure and the utility function...... 82

Appendix 1b. Responses of the Fish and Wild life Service members of the Science Team for the curve exercise designed to elicit information necessary to determine the vegetation state structure and part of the utility function.

Appendix 1c. Description of the method used to determine the vegetation state structure based on the elicited responses to the curve exercise.

Appendix 1d. Description of the rating exercise designed to elicit information necessary to determine the utility function from the Fish and Wildlife Service members of the Science Team.

Appendix 1e. Responses of the Fish and Wildlife Service members of the Science Team for the rating exercise designed to elicit information necessary to determine the utility function.

Appendix 1f. Description of the method used to determine the utility based on the elicited responses to the curve exercise and the rating exercise. 
Appendix 2. Outline of the provisional Native Prairie Adaptive Management (NPAM) decision framework; the first complete, functional, but provisionary, version of the NPAM decision framework used to complete the first adaptive management cycle during management year 2010|2011..................................... 105

Appendix 3a. Model elicitation spreadsheet approach and rationale for mixed-grass baseline model. ................. 111

Appendix 3b. Model elicitation spreadsheet approach and rationale for tallgrass baseline model. ....................... 119

Appendix 4a1. Elicitation response for mixed-grass Model 3 ................................................................ 127

Appendix 4a2. Modification of the mixed-grass Model 3 elicitation response to provide a response for mixed-grass Model 1 ............................................................................................................. 129

Appendix 4a3. Modification of the mixed-grass Model 3 elicitation response to provide a response for mixed-grass Model 2 ............................................................................................................... 130

Appendix 4a4. Modification of the mixed-grass Model 3 elicitation response to provide a response for mixed-grass Model 4.

Appendix 4b1. Elicitation response for tallgrass Model 3.

Appendix 4b2. Modification of the tallgrass Model 3 elicitation response to provide a response for tallgrass Model 1.

Appendix 4b3. Modification of the tallgrass Model 3 elicitation response to provide a response for tallgrass Model 2.

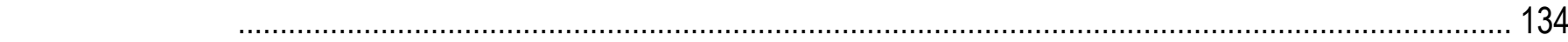

Appendix 4b4. Modification of the tallgrass Model 3 elicitation response to provide a response for tallgrass Model 4.

Appendix 4b5. Modification of the tallgrass Model 3 elicitation response to provide a response for tallgrass Model 5.

Appendix 4b6. Modification of the tallgrass Model 3 elicitation response to provide a response for tallgrass Model 6 .

Appendix 5a. Modifications to the mixed-grass baseline model elicitation spreadsheet to derive values for the alternative models. ................................................................................................... 138

Appendix 5b. Modifications to the tallgrass baseline model elicitation spreadsheet to derive values for the

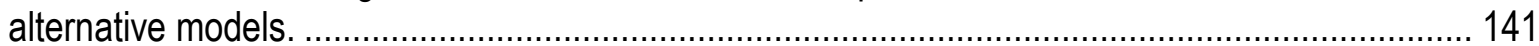

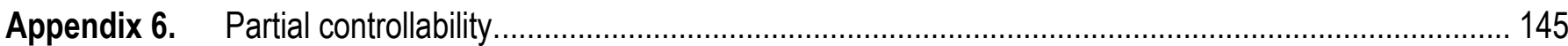

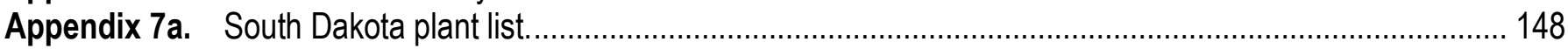

Appendix 7b. North Dakota plant list. ............................................................................................. 149

Appendix 8a. Summary of system state and management of mixed-grass units from cooperating stations from project inception (2009) through 2012 ....................................................................... 150

Appendix 8b. Summary of system state and management of tallgrass units from cooperating stations from project

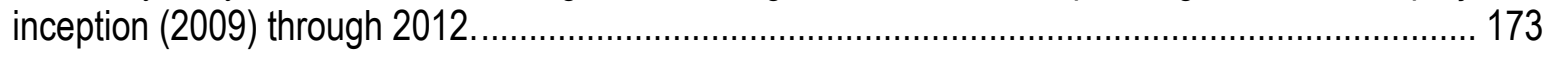




\section{Acknowledgments}

Funding for this study was furnished by the Refuge Cooperative Research Program, Northern Prairie Wildlife Research Center, Patuxent Wildlife Research Center, and Georgia Cooperative Fish and Wildlife Research Unit of the U.S. Geological Survey (USGS).

Other funding for this study was furnished by regions 3 and 6 , and the Inventory and Monitoring Program of the U.S. Fish and Wildlife Service (FWS); and the Plains and Prairie Potholes Landscape Conservation Cooperative.

We thank several USGS personnel. Lianne Ball provided her support of the Native Prairie Adaptive Management (NPAM) initiative. William Link furnished technical input into statistical models. Thomas Buhl coordinated and handled logistics for numerous cooperator meetings. James Peterson and Amy Symstad provided helpful comments on earlier versions of the manuscript.

We also thank several non-USGS personnel. We thank Patricia Heglund, Wayne King, Melinda Knutson, and Socheata Lor from FWS for their unwavering support of NPAM. Bob Patton (North Dakota State University), James Stubbendieck (University of Nebraska), and Gene Towne (Kansas State University) provided access to legacy data. Justin Dupey (FWS), Victoria Hunt (Chicago Botanic Garden; CBG), Sarah Jacobi (CBG), Eric Lonsdorf (CBG), Kevin McAbee (FWS), Todd Sutherland (FWS), and Jennifer Zorn (FWS) contributed to development and maintenance of the database management system. We especially thank Kim Bousquet, Cami Dixon, Pauline Drobney, Vanessa Fields, Bridgette Flanders-Wanner, Todd Grant, and Sara Vacek for their invaluable contributions to the project as FWS members of the NPAM Science Team. Special thanks to Cami Dixon and Bridgette Flanders-Wanner for serving as the NPAM Project Coordinator. Finally, we thank the NPAM FWS cooperators for their invaluable input and patience at cooperator meetings and for managing prairies and monitoring the outcome of that management. 


\title{
Native Prairie Adaptive Management: A Multi Region Adaptive Approach to Invasive Plant Management on Fish and Wildlife Service Owned Native Prairies
}

\author{
By Jill J. Gannon, Terry L. Shaffer, and Clinton T. Moore
}

\section{Executive Summary}

Much of the native prairie managed by the U.S. Fish and Wildlife Service (FWS) in the Prairie Pothole Region (PPR) of the northern Great Plains is extensively invaded by the introduced cool-season grasses, smooth brome (Bromus inermis) and Kentucky bluegrass (Poa pratensis). Management to suppress these invasive plants has had poor to inconsistent success. The central challenge to managers is selecting appropriate management actions in the face of biological and environmental uncertainties. In partnership with the FWS, the U.S. Geological Survey (USGS) developed an adaptive decision support framework to assist managers in selecting management actions under uncertainty and maximizing learning from management outcomes. This joint partnership is known as the Native Prairie Adaptive Management (NPAM) initiative. The NPAM decision framework is built around practical constraints faced by FWS refuge managers and includes identification of the management objective and strategies, analysis of uncertainty and construction of competing decision models, monitoring, and mechanisms for model feedback and decision selection. Nineteen FWS field stations, spanning four states of the PPR, have participated in the initiative. These FWS cooperators share a common management objective, available management strategies, and biological uncertainties. Though the scope is broad, the initiative interfaces with individual land managers who provide site-specific information and receive updated decision guidance that incorporates understanding gained from the collective experience of all cooperators. We describe the technical components of this approach, how the components integrate and inform each other, how data feedback from individual cooperators serves to reduce uncertainty across the whole region, and how a successful adaptive management project is coordinated and maintained on a large scale.

During an initial scoping workshop, FWS cooperators developed a consensus management objective: increase the composition of native grasses and forbs on native sod while minimizing cost. Cooperators agreed that decision guidance should be provided annually and should account for local, real-time vegetation conditions observed on the ground. Over the course of development, two prototypes of the decision framework were considered. The final framework recognized four alternative actions that managers could take in any given year: (1) Graze - targeted use of grazing ungulates to achieve defoliation, (2) Burn - application of prescribed fire as the single form of defoliation, (3) Burn/Graze - a combination treatment, and (4) Rest — no action. The study area included northern mixed-grass and tallgrass prairie. Native vegetation in mixed-grass prairie has a strong cool-season component and thus the dominant native species have a phenology similar to that of smooth brome and Kentucky bluegrass, making management of those species challenging. In contrast, tallgrass prairie has a strong warm-season native component, leading to an existence of cool-season windows, periods of time in the fall and spring when cool-season invasive grass species are actively growing and vulnerable to damage via select management actions, but warm-season grass species are not active and are thus less 
susceptible to damage via the same actions. This dichotomy between prairie types necessitated the development of separate but parallel decision support systems for mixed-grass and tallgrass biomes.

Management units are parcels of native prairie that receive a single management treatment at any one time over their entire extent. At any particular time, the vegetation state of each management unit is characterized by the amount of cover of native grasses and forbs and the type of invasive grass that is dominant. In addition, each unit has a defoliation state which reflects the number of years since the last defoliation event and an index to how intensively the unit was managed during the previous 7 years. State-transition models are used to predict the state of a management unit in year $t+1$ from its state in year $t$ and a prescribed management action that was applied between the two monitoring events. Alternative models are built around key uncertainties that make choice of a management action difficult. Three uncertainties revolve around whether the effect of management actions depends on (1) type of dominant invader, (2) past defoliation history, and (3) level of invasion. Two additional uncertainties are considered when choosing a management action for tallgrass units: (4) the effectiveness of grazing within the cool-season window as a surrogate for burning when smooth brome is the dominant invader, and (5) the differential effect of active management outside the window as compared to rest.

Because data on the probability of transitioning from one state to another under the various models were lacking, expert opinion and elicitation were used to parameterize the models. In addition, cooperators participated in elicitation exercises to extract their beliefs regarding the value of having native prairie compared to the cost of achieving it. Quantifying the subjective expression of utility in this way allowed for mathematical representation of the management objective into an objective function. By maximizing the objective function, cumulative utility is maximized, leading to the identification of a sequence of decisions that will achieve the management objective.

The NPAM system adopted a vegetation monitoring protocol that was rapid, inexpensive, and familiar to many of the cooperators. The monitoring protocol served three purposes: (1) determining current vegetation and defoliation states of each unit, (2) evaluating progress toward the management objective, and (3) assessing predictive performance of the alternative models. The management year runs from September 1 to August 31. Management can be applied anytime during that period and monitoring takes places from late June to mid-August. Cooperators enter vegetation data and management information into a centralized database by August 25 of each year. Given the current state of the system (vegetation and defoliation states) and the current understanding of the system (or the belief state), identifying the current best management decision is a matter of looking up the combination (that is, system state and belief state) in the appropriate (mixed-grass or tallgrass) optimal decision table. Given complete uncertainty at the outset of decision-making, initial assignment of equal belief weights to each model was believed reasonable. The decisions in the optimal decision table that correspond to the current belief state constitute the current optimal decision policy. By August 31 of each year, individual cooperators are provided with a recommended management action for each of their management units for the upcoming management year. Upon receiving the management recommendations for their units, managers consider the recommendation, along with other relevant information, and at some point during the year one of the management alternatives is carried out. This iterative cycle of making and implementing a management decision, predicting the response, monitoring the outcome, comparing predicted and observed outcomes, updating model weights, and recommending a management action for the next cycle is expected to result in an accumulation of weight on a representative model of system dynamics, thereby increasing understanding needed to effectively manage native prairies.

The NPAM system is now entering its second full year of complete operation, and represents one of only a few fully implemented applications of adaptive management within the U.S. Fish and 
Wildlife Service. NPAM is truly unique in that it originated from the ground up as a result of the leadership and steadfastness of several refuge biologists and managers confronted with a common problem. These biologists recognized that working together across a large landscape presented perhaps the best opportunity for halting and reversing the invasion of native grasslands by non-native coolseason grasses. Importantly, the NPAM system encapsulates the collective thinking and experience of tens if not hundreds of individuals who have battled this vexing problem for much of their careers.

The NPAM initiative is rooted in principles of adaptive management, thereby affording the opportunity for grassland managers to pursue management objectives while acquiring information to reduce uncertainty and improve future management. The project introduced a number of technical innovations that will serve as templates for conservation efforts throughout and beyond the U.S. Fish and Wildlife Service. First, NPAM is an on-the-ground implementation of active adaptive management - possibly the first of its kind in conservation management - in which recommended management actions result from a prospective analysis of future learning (Williams, 1996). Second, by the use of dynamic optimization, NPAM demonstrates how decisions can be made that take into account possible future transitions of the system. Third, NPAM demonstrates how models of partial controllability are an effective means of accommodating unpredictable circumstances that cause a manager to follow a different course than was intended. Finally, the database developed for NPAM is an unparalleled system that enables the rapid integration of data from the field for the generation of 'just-in-time' management recommendations. In all, NPAM provides an example of how a sciencemanagement partnership can be forged to achieve large-scale conservation objectives.

\subsection{Introduction}

Grassland biomes in North America, exemplified mainly by the vast Great Plains, are arguably the most endangered major ecosystem on the continent (Samson and Knopf, 1994; Samson and others, 2004). The decline in extent and quality of North American prairies coincides with decreasing populations of many animal species that depend on them, and is among the most challenging conservation issues of this century (Samson and others, 2004; Brennan and Kuvlesky, 2005). In states and provinces of the northern prairie region, for example, native mixed-grass prairie has declined from 30 to 99 percent and native tallgrass prairie has declined more than 95 percent, mainly because of conversion of prairie to agriculture (Samson and others, 2004). This trend continues unabated for prairies in North Dakota and South Dakota (Higgins and others, 2002).

More than 100,000 hectares (ha) of native prairie lands are found in the Prairie Pothole Region (PPR) within the collection of National Wildlife Refuge System (NWRS) lands of the U.S. Fish and Wildlife Service (FWS), which is charged with managing this large public land base (Grant and others, 2009). Given the decline of this ecosystem throughout the PPR, these refuge lands have become increasingly important conservation reservoirs for native prairie. In 2005, region 6 of the FWS established the Dakota Grassland Monitoring Team (DGMT). The DGMT was formed to address biological support for grassland management on NWRS lands in the Dakotas. The primary charge of the DGMT was to develop methods that facilitate inventory, monitoring, and applied research for addressing management issues in grasslands. The DGMT queried managers and biologists regarding management issues and threats to grasslands on National Wildlife Refuges (NWR) in North Dakota and South Dakota. Invasion of native prairies by smooth brome (Bromus inermis) was the most common threat identified for the region. This rhizomatous, introduced grass rapidly out-competes native grasses and forbs, especially under extended periods of rest, and forms monotypic stands that lack structural variety and species diversity (Oftinowski and others, 2007). Invasions of FWS-owned prairies by 
smooth brome and other cool-season exotic species, especially Kentucky bluegrass (Poa pratensis) are believed to stem, in part, from a common management history (circa 1935-85) of long-term rest and little or no defoliation by natural processes [that is, year-round grazing by native ungulates and an approximate 5-year fire return interval (Murphy and Grant, 2005)] that historically shaped native vegetation communities (Grant and others, 2009).

Historic floras (that is, pre-EuroAmerican settlement) of the northern prairie region have been described broadly (Stubbendieck, 1988; Coupland, 1992). However, comprehensive assessments of the current flora of northern prairie refuges have not been undertaken until recently; an extensive inventory of prairie on five refuge complexes in the Drift Plain physiographic subregion of the northern mixedgrass prairie was completed by Grant and others (2009). Prairies were badly invaded by introduced cool-season grasses, especially smooth brome, Kentucky bluegrass, and native species of shrub [e.g., western snowberry (Symphoricarpos occidentalis) and silverberry (Elaeagnus commutate)]. Although many factors probably contributed to the deterioration of the prairie, similarity among complexes in conditions of the vegetation seemed to parallel their common management histories of mainly long-term rest (that is, no disturbance) or light, season-long grazing (Murphy and Grant, 2005; Grant and others, 2009).

Management attempts to suppress smooth brome, Kentucky bluegrass, and other cool-season invasive species [e.g., Canada thistle (Cirsium arvense)] have met with poor to inconsistent success, particularly in prairies naturally dominated by cool-season native plants (e.g., North Dakota) and where extended rest characterizes the management history. Strategies for shifting plant competition to favor native over introduced species are poorly understood in northern mixed-grass prairies. Initial efforts by NWR staff to learn more were sporadic and lacked scientific rigor, with each field station informally exploring the issue independent of their counterparts. Recognition of the seriousness of these issues prompted the DGWT to hold a meeting (referred to as the Brome Summit) in March 2006 (U.S. Fish and Wildlife Service, 2006). The two-day technical meeting was attended by 30 individuals with expertise on smooth brome from federal and state agencies, universities, and the private sector. Attendees exchanged and synthesized information on smooth brome ecology and control strategies. The summit revealed major knowledge gaps and uncertainties about effective strategies for reducing the spread of smooth brome.

The Brome Summit further revealed that a solution to the invasive-species problem would likely have to come from within the FWS. That realization spurred the DGMT to recommend, and NWRs in the Dakotas to later embrace, a collaborative approach to the restoration of FWS-owned native prairies in the Dakotas based on the application of adaptive management as a means for reducing management uncertainty. By developing a structured process for guiding and evaluating management of native prairies across North and South Dakota, the DGMT believed that results would have broader applicability and greater reliability than any one field station could independently achieve. The DGMT further believed that this type of adaptive, collaborative process could be used to address other significant habitat management issues common to NWRs and other public or private grasslands in the region. The DGMT agreed, however, that initial development of an adaptive management system should focus on strategies to restore native prairies on NWRs and specifically on methods to reduce and reverse the spread of smooth brome.

At about the same time the DGMT was considering how to approach restoration of cool-season native prairies in the Dakotas, the Minnesota Grassland Monitoring Team (MGMT) was being founded in FWS region 3. The original purpose of the MGMT was to organize and develop protocols for collecting baseline data on the status of native tallgrass prairies in states that comprise the FWS region 3. The MGMT included natural resource managers and biologists from several agencies working in 
western Minnesota. The group formed with the goal of developing a standardized, hierarchical monitoring protocol that would enable individual grassland monitoring programs to pool monitoring resources and data toward a common purpose.

When, in 2007, MGMT partnered with the NWRS Biological Monitoring Team to hold an adaptive management consultancy aimed at developing a decision support system for managing native tallgrass prairies in region 3, the missions of the DGWT and the MGMT aligned. Thus, the potential to work together and capitalize on the collective resources and expertise in both regions was soon recognized. Out of that recognition emerged the multi region Native Prairie Adaptive Management (NPAM) initiative described here. Primary funding for development of the NPAM system was secured in early 2008 from the U.S. Geological Survey (USGS) Refuge Cooperative Research Program (RCRP). RCRP is a partnership involving the FWS NWRS and USGS and champions the development and application of multi refuge, multi region, learning-based decision support tools, and thus was a natural fit for the NPAM project.

\subsection{Study Area}

The NPAM initiative focuses on FWS NWRS lands within the PPR of the northern Great Plains (fig. 1; table 1). Within the PPR, 19 refuge complexes and wetland management districts (hereafter referred to as refuges, stations, or cooperators) contributed 120 management units to the initiative. Management units are parcels that receive a single management treatment at any one time over their entire extent; average unit size was 35 ha (range 3.5-241 ha). These units span the boundaries of two FWS regions (3 and 6), four states (North Dakota, South Dakota, Minnesota, and Montana), and two major grassland types known as mixed-grass and tallgrass prairie (Samson and others, 1998).

Tallgrass prairie is the wettest of northern Great Plains grassland types and is predominantly composed of sod-forming grasses (Samson and others, 1998). Precipitation [64-102 centimeters $(\mathrm{cm})$ annually] falls mostly during spring and summer (Bailey, 1978). Tallgrass prairie of western Minnesota and eastern North Dakota has been characterized as bluestem prairie (Samson and others, 1988). Native vegetation of the tallgrass prairie has a strong warm-season component; therefore phenology of cool-season smooth brome and Kentucky bluegrass generally does not overlap that of dominant native grasses. Common warm-season species include big bluestem (Andropogon gerardii), Indiangrass (Sorghastrum nutans), and switchgrass (Panicum virgatum) (Bailey, 1978; Risser and others, 1981). To the west, the drier northern mixed-grass prairie of western and central North Dakota and South Dakota primarily consists of cool-season grasses with a variable mix of warm-season species. Native grasses include several cool-season grasses, primarily needlegrasses (Stipa spp.) and wheatgrasses (Agropyron spp.), and several warm-season grasses, mainly blue grama (Bouteloua gracilis), sideoats grama ( $B$. curtipendula) and bluestem (A. gerardii and Schizachyrium scopairum) species (Grant and others, 2009). Annual precipitation of 40-50 cm falls primarily during the growing season, and droughts are common. Because of precipitation differences, it is common for grasses of tallgrass prairie to reach heights of 100-300 cm, whereas grasses of the mixed-grass prairie tend to be shorter $(60-122 \mathrm{~cm})$ (Risser and others, 1981). 


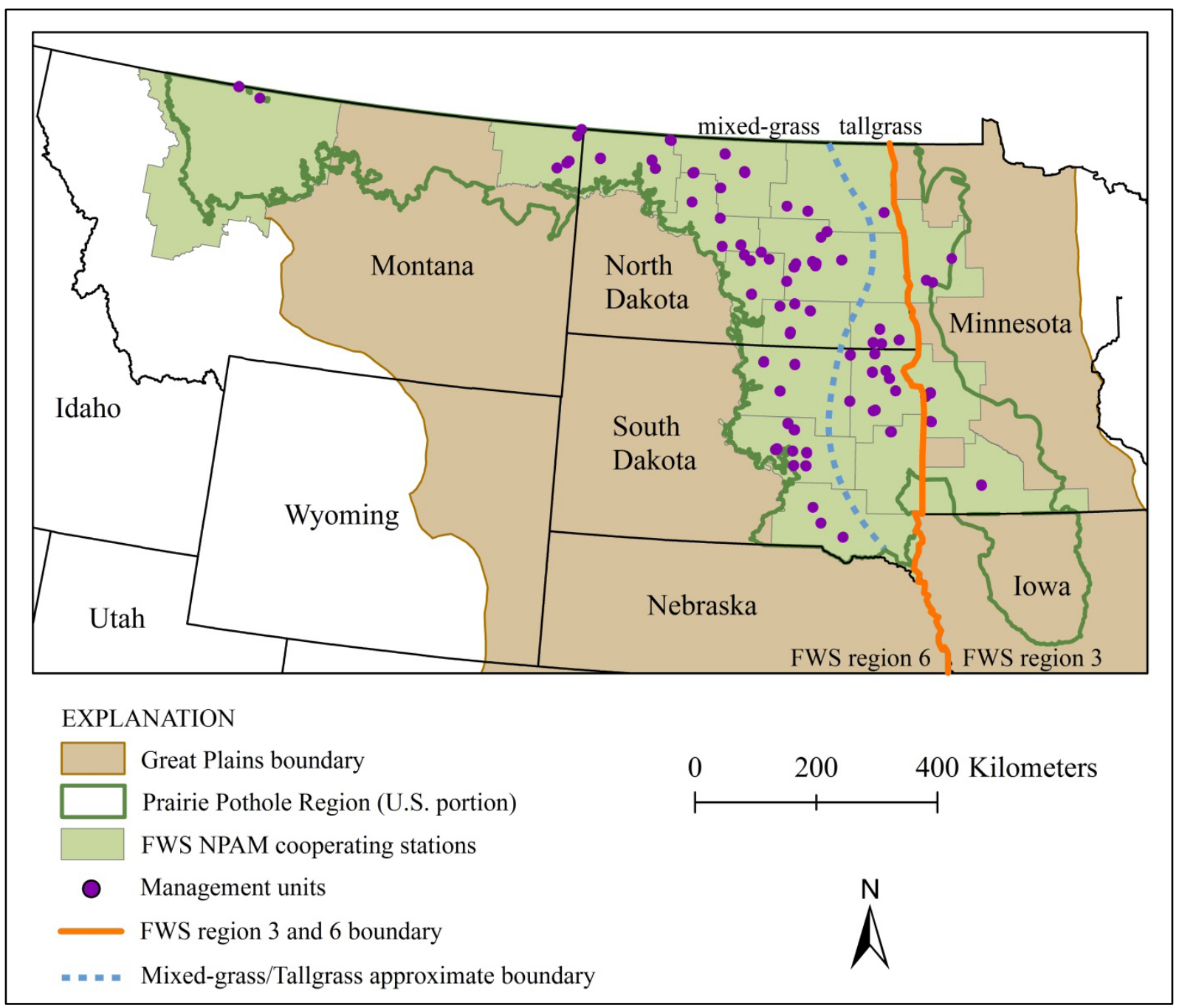

Figure 1. The Native Prairie Adaptive Management area of focus is native sod, on U.S. Fish and Wildlife Service (FWS)-owned lands, across the Prairie Pothole Region (PPR), within FWS regions 3 and 6. 
Table 1. The 19 U.S. Fish and Wildlife Service (FWS) Native Prairie Adaptive Management (NPAM) cooperators, number of management units each cooperator contributed to NPAM, and the grass type, state, and FWS administrative region of the unit.

[NWR=National Wildlife Refuge. WMD=Wetland Management District. ND=North Dakota. MT=Montana. $\mathrm{MN}=$ Minnesota. $\mathrm{SD}=$ South Dakota]

\begin{tabular}{|c|c|c|c|c|}
\hline Cooperating station & $\begin{array}{l}\text { Number } \\
\text { of units }\end{array}$ & $\begin{array}{l}\text { Grass } \\
\text { type }\end{array}$ & State & $\begin{array}{c}\text { FWS } \\
\text { region }\end{array}$ \\
\hline Arrowwood NWR Complex & 16 & Mixed & ND & 6 \\
\hline Audubon NWR Complex & 11 & Mixed & ND & 6 \\
\hline Benton Lake WMD & 2 & Mixed & MT & 6 \\
\hline Big Stone NWR & 1 & Tall & MN & 3 \\
\hline Detroit Lakes WMD & 3 & Tall & MN & 3 \\
\hline Devils Lake WMD & $\begin{array}{l}2 \\
1\end{array}$ & $\begin{array}{l}\text { Mixed } \\
\text { Tall }\end{array}$ & ND & 6 \\
\hline Huron WMD & 10 & Mixed & SD & 6 \\
\hline Kulm WMD & 10 & Mixed & ND & 6 \\
\hline Lake Andes NWR & 3 & Mixed & SD & 6 \\
\hline Long Lake WMD & 5 & Mixed & ND & 6 \\
\hline Lostwood NWR Complex & 3 & Mixed & ND & 6 \\
\hline Madison WMD & 3 & Tall & SD & 6 \\
\hline Medicine Lake NWR & 6 & Mixed & MT & 6 \\
\hline Morris WMD & 9 & Tall & MN & 3 \\
\hline Sand Lake NWR Complex & $\begin{array}{l}3 \\
2 \\
\end{array}$ & $\begin{array}{l}\text { Mixed } \\
\text { Tall }\end{array}$ & SD & 6 \\
\hline $\begin{array}{l}\text { Souris River Basin NWR } \\
\text { Complex }\end{array}$ & 10 & Mixed & ND & 6 \\
\hline Tewaukon WMD & 7 & Tall & ND & 6 \\
\hline Waubay NWR Complex & 11 & Tall & SD & 6 \\
\hline Windom WMD & 2 & Tall & $\mathrm{MN}$ & 3 \\
\hline
\end{tabular}




\subsection{Adaptive Management Decision Support Framework}

Given the focus of the RCRP (multi refuge, learning-based decision support), we established the general design of the decision framework around recognition that decision support and system learning should be treated hierarchically. That is, we envisioned a framework that would regularly distribute decision support outward to a collection of cooperators (managers at participating FWS stations) across the landscape, where decision guidance would be tailored to current local conditions. At the same time, the framework would centrally collect observed responses to management and synthesize the information into updated knowledge about general function of the system.

Our work began with the hiring of a USGS postdoctoral research associate and the establishment of a USGS-FWS Science Team. The Science Team comprised the USGS principal investigators, the research associate, and six FWS refuge biologists, three from each region. The Science Team played several roles; the main role was to make strategic decisions about how the project should proceed and how the decision framework should be structured. In a number of areas where the framework lacked quantification, the FWS members of the Science Team were called upon to participate in exercises to elicit values-based (e.g., section 4.2.2 Utility) or scientific judgments (e.g., section 4.5.1.2 Model Parameterization) that were inserted into the framework. The FWS Science Team members also served as representatives of their peers at cooperating stations. Thus, the abilities, constraints, needs, and expectations of managers were communicated to the USGS Science Team members. Finally, one of the FWS Science Team members served as project coordinator, who communicated schedules, protocols, updates, and requests for information to station cooperators.

During an initial meeting with interested cooperators in 2008, and in meetings held throughout the following months, the Science Team came to agreement on several strategies and principles that would define our approach for developing the decision framework. First, we affirmed that any framework must be built around a hierarchical arrangement of distributed decision guidance and centralized learning. Cooperators were made aware that their participation paid not only immediate dividends to themselves (in the form of timely decision guidance) but also future dividends to themselves and to their peers (in the form of increased understanding about prairie systems and improved decision making).

Second, we agreed that decision guidance should be provided to cooperators annually and should account for local, real-time vegetation conditions observed on the ground. That is, the decision guidance provided to managers each year should be state specific. In early discussions, we considered a biennial time step for decision making, in which a two-year prescription for action would be provided every other year, coinciding with a biennial program of monitoring. However, managers expressed strong interest in receiving decision guidance responsive to annual dynamic conditions, and they had the capability to provide the requisite annual monitoring support.

Third, recognizing that biological systems and approaches to prairie management differed substantially across the study region, we believed it important to develop parallel but distinct decision support systems for cooperators within the tallgrass and mixed-grass biomes. We acknowledged that prairie system dynamics are differentially responsive across other gradients (principally soils and climate), but those differences are unknown and difficult to quantify. Instead, we believed that stratification of the study region into the two grassland biomes and developing decision frameworks for each would control for much of this across-region variation and would be feasible to accomplish.

Fourth, we recognized how uncertainty often has impeded past approaches to prairie management. Structural uncertainty, or uncertainty about the mean response of a system to management action, is a form of uncertainty that potentially is reducible through the use of predictive modeling and data feedback (that is, adaptive management) (Walters, 1986; McCarthy and Possingham, 
2007; Williams and others, 2007; Runge, 2011). Environmental stochasticity, partial controllability, and partial observability are three other forms of uncertainty that are largely irreducible but that nevertheless can detrimentally affect management outcomes if not taken into account (Nichols and others, 1995; Williams, 2001). We intended the framework to address uncertainty through three strategies: (1) accounting for structural uncertainties and attempting to reduce them over time through application of adaptive management principles; (2) accounting for the effects of irreducible uncertainties on management decisions by modeling these phenomena within optimization and updating routines; and (3) systematically collecting management treatment data and vegetation response data, making feasible post hoc analyses to refine the decision framework over time.

Finally, we agreed that emphasis should be placed on the construction of a coherent and sustainable decision framework that could be parameterized with existing data or expert judgment. To us, this meant that identifying the framework elements at the outset would indicate what types of information would be needed to parameterize the system. The hope was that quantitative information could come from meta-analytic investigation of related datasets or from carefully elicited expert judgments; in no case would we use field experimentation to parameterize the decision models. Even in cases where some values are merely best guesses serving only as placeholders, the expectation is that empirical data will accrue through time as part of the monitoring effort and eventually displace the guessed values.

Two distinct phases are usually identified in adaptive management efforts: a finite setup or deliberative phase, and an indefinite iterative or implementation phase (Williams and others, 2007). The setup phase pertains to all the work in constructing the decision-making framework, including the set of protocols and tools that are needed to sustain the iterative phase of the effort. The iterative phase pertains to the routine cycle of monitoring, applying a decision action, and updating knowledge about the system. Under the RCRP agreement between the USGS and FWS, the USGS-FWS partnership works together through the setup phase to build the decision framework. The FWS then takes responsibility for carrying out the iterative phase. This report provides information on tasks accomplished during the setup phase within the partnership. We also provide summary information on the first experimental cycles of the iterative phase that were carried out during the setup work.

\subsection{Setup Phase}

\subsection{Bounding the Problem}

The resource problem on which the NPAM framework is focused is the loss of native prairie to two cool-season invasive grass species, smooth brome and Kentucky bluegrass. The area in which the NPAM framework is focused is native sod, on FWS-owned refuge lands, across the Prairie Pothole Region, within FWS regions 3 and 6 where smooth brome and Kentucky bluegrass are the main invasive species of concern (fig. 1; table 1). The spatial unit of focus is the management unit, which is defined as a parcel of land that receives a single management action on its entire extent. The management objective of NPAM is to increase the cover of native grasses and forbs at the least cost; the objective is detailed in section 4.2 Management Objective. The set of alternative management actions that can be used to pursue the objective includes rest, graze, burn, and burn/graze for mixed-grass and rest, graze within window, burn within window, and defoliate for tallgrass; these actions are described in detail in section 4.3 Management Alternatives. The decision cycle for deciding on and implementing an action from the set of alternative actions and conducting follow-up monitoring is the management year, which is defined as September 1 to August 31 (fig. 2). The management problem, then, can be 
recast as the following decision question: Which is the best management action to implement each year to decrease cool-season invasive grass species and increase the cover of native grasses and forbs on each unit, while minimizing cost?

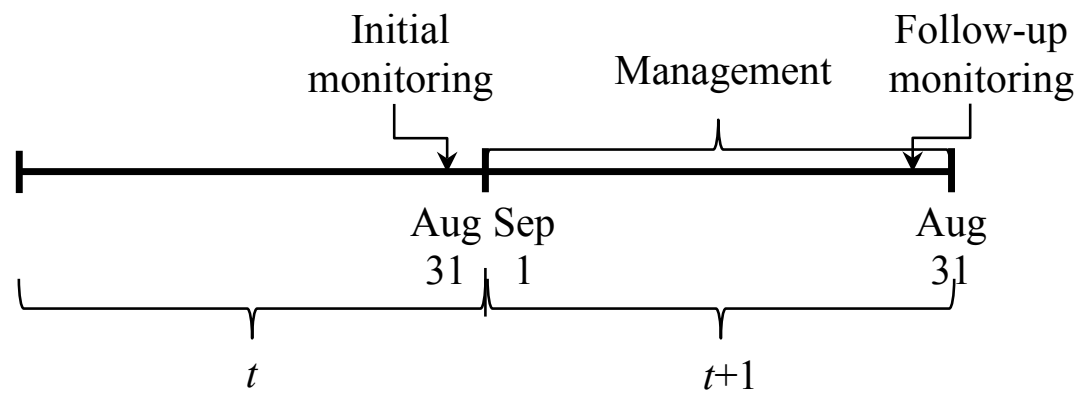

Figure 2. Depiction of the management year, which runs from September 1 to August 31. Vegetation is initially monitored on a unit when it is first enrolled in Native Prairie Adaptive Management; this initial monitoring is denoted as taking place in year $t$. The following management year is denoted as $t+1$. The management year includes the implementation of the selected management action on the unit, which typically occurs in the fall or spring (or both), as well as the follow-up vegetation monitoring, which is completed during the growing season (June-August) and measures the system response to the implemented management action.

\subsection{Management Objective}

\subsubsection{General Statement}

Under adaptive management, the selection of decisions is driven by an explicit, measurable management objective (Williams and others, 2002). The objective statement must (1) be quantifiable and measureable in the field, (2) define a quantity that can be generated as output from a decision model, and (3) balance tradeoffs among multiple objectives. One of our first tasks was to hold an initial, facilitated problem-scoping workshop to define the management objective. The workshop was held in July 2008 and was attended by 25 FWS personnel (managers, biologists, project leaders) representing 19 different refuges from across the PPR of FWS regions 3 and 6. During the workshop, participants considered various management goals such as increasing and maintaining native grass and forb species diversity, structural diversity, ecosystem function, and focal trust species (e.g., ducks). After thorough discussion, the group decided that the principal concern is to restore and maintain native cover and they developed the consensus management-objective statement to increase the composition of native grasses and forbs on native sod while minimizing cost (digital appendix 1).

\subsubsection{Utility}

Utility describes what managers want out of the system through management. What managers want, the management objective, is to increase the cover of native grasses and forbs while minimizing cost. The utility combines both aspects of the management objective by expressing the balance between the value of having native prairie with the cost of achieving it. That is, utility takes into account the importance of having native grass and forb cover (the desired natural resource), along with the willingness to direct monetary resources to obtain the desired natural resource. 
We treated utility as a constructed attribute (Keeney, 1992) to express the desirability of, or the level of satisfaction with, an outcome, where the outcome is the resulting native cover after implementing a given management action. A utility of ' 0 ' is the least satisfied one can be, whereas a utility of ' 1 ' is the most satisfied one can be with the outcome. Using utility values allows us to assess the value of two different resources, natural and monetary, that have two different natural measurable attributes, percent of native cover present and dollars spent, on the same constructed scale of measurement, desirability. In doing so, we are able to simultaneously assess the two aspects by combining them into a single utility value.

Utility is a subjective expression of how managers feel about the response of the system (in terms of the amount of native cover) to the management actions they implement. As such, parameterizing the utility is a process that stands completely apart from the construction of the model set, which makes predictions about the behavior of the system and how it responds to management. We determined the utility values through an elicitation exercise with the FWS members of the Science Team. The utility-parameterization exercise followed a preliminary exercise to establish the vegetation state structure that framed the utility values (section 4.4.1 Vegetation State; appendixes 1a, 1b, 1c). Details of the exercise, responses, and method used to arrive at the final utility values are provided in appendixes 1d, 1e, and $1 \mathrm{f}$. The complete utility (table 2) consists of a single utility matrix for each alternative management action.

Table 2. A, Complete utility for mixed-grass prairie management units, including a single utility matrix for each alternative management action: rest, graze, burn, and burn/graze. $B$, Complete utility for tallgrass prairie management units, including a single utility matrix for each alternative management action: rest, graze within window, burn within window, and defoliate.

[The unit of native cover is percent. Time $t$ refers to the current year, whereas time $t+1$ denotes the subsequent year; that is, the matrices represent a single annual time step. The shading within the matrices serves to divide them into three partitions (along the diagonal, below the diagonal, and above the diagonal), the relevance of which is described in the text. When comparing between the mixed-grass and tallgrass utilities, the rest matrices are the same, the graze and graze within window matrices are the same, and the burn and burn within window matrices are the same. The difference between the utilities of the two grass types is the presence of the burn/graze matrix in mixed-grass and the defoliate matrix in tallgrass]

$A$, Mixed-grass

\section{$\underline{\text { Rest }}$}

Native cover at time $t+1$

\begin{tabular}{cr|c|c|c|c|} 
& & $60-100$ & $45-60$ & $30-45$ & $0-30$ \\
\cline { 3 - 6 } Native & $60-100$ & 0.92 & 0.61 & 0.35 & 0.18 \\
\cline { 3 - 6 } cover at & $45-60$ & 0.95 & 0.75 & 0.51 & 0.29 \\
\cline { 3 - 6 } time $t$ & $30-45$ & 0.96 & 0.79 & 0.59 & 0.37 \\
\cline { 3 - 6 } & $0-30$ & 0.97 & 0.82 & 0.64 & 0.44 \\
\cline { 3 - 6 } & & & & &
\end{tabular}

Graze

Native cover at time $t+1$

\begin{tabular}{cr|c|c|c|c|} 
& & $60-100$ & $45-60$ & $30-45$ & $0-30$ \\
\cline { 3 - 6 } Native & $60-100$ & 0.87 & 0.55 & 0.29 & 0.13 \\
\cline { 3 - 6 } cover at & $45-60$ & 0.90 & 0.69 & 0.43 & 0.21 \\
\cline { 3 - 6 } time $t$ & $30-45$ & 0.91 & 0.72 & 0.52 & 0.28 \\
\cline { 3 - 6 } & $0-30$ & 0.92 & 0.75 & 0.56 & 0.36 \\
\cline { 3 - 6 } & & & &
\end{tabular}


$\underline{\text { Burn }}$

Native cover at time $t+1$

\begin{tabular}{cr|c|c|c|c|} 
& & $60-100$ & $45-60$ & $30-45$ & $0-30$ \\
\cline { 3 - 6 } Native & $60-100$ & 0.83 & 0.50 & 0.25 & 0.10 \\
\cline { 3 - 6 } cover at & $45-60$ & 0.86 & 0.64 & 0.37 & 0.16 \\
\cline { 3 - 6 } time $t$ & $30-45$ & 0.88 & 0.67 & 0.46 & 0.22 \\
\cline { 3 - 6 } & $0-30$ & 0.89 & 0.70 & 0.50 & 0.29 \\
\cline { 3 - 6 } & & & & &
\end{tabular}

$B$, Tallgrass

$\underline{\text { Rest }}$

Native cover at time $t+1$

\begin{tabular}{cr|c|c|c|c|} 
& & $60-100$ & $45-60$ & $30-45$ & $0-30$ \\
\cline { 3 - 6 } Native & $60-100$ & 0.92 & 0.61 & 0.35 & 0.18 \\
\cline { 3 - 6 } cover at & $45-60$ & 0.95 & 0.75 & 0.51 & 0.29 \\
\cline { 3 - 6 } time $t$ & $30-45$ & 0.96 & 0.79 & 0.59 & 0.37 \\
\cline { 3 - 6 } & $0-30$ & 0.97 & 0.82 & 0.64 & 0.44 \\
\cline { 3 - 6 } & & & &
\end{tabular}

Graze within window

Native cover at time $t+1$

\begin{tabular}{cr|c|c|c|c|} 
& & $60-100$ & $45-60$ & $30-45$ & $0-30$ \\
\cline { 3 - 6 } Native & $60-100$ & 0.87 & 0.55 & 0.29 & 0.13 \\
\cline { 3 - 6 } cover at & $45-60$ & 0.90 & 0.69 & 0.43 & 0.21 \\
\cline { 3 - 6 } time $t$ & $30-45$ & 0.91 & 0.72 & 0.52 & 0.28 \\
\cline { 3 - 6 } & $0-30$ & 0.92 & 0.75 & 0.56 & 0.36 \\
\cline { 3 - 6 } & & & & &
\end{tabular}

Burn/Graze

Native cover at time $t+1$

\begin{tabular}{cr|c|c|c|c|} 
& & $60-100$ & \multicolumn{1}{c}{$45-60$} & $30-45$ & $0-30$ \\
\cline { 3 - 6 } Native & $60-100$ & 0.81 & 0.47 & 0.22 & 0.07 \\
\cline { 3 - 6 } cover at & $45-60$ & 0.83 & 0.61 & 0.33 & 0.12 \\
\cline { 3 - 6 } time $t$ & $30-45$ & 0.85 & 0.64 & 0.42 & 0.17 \\
\cline { 3 - 6 } & $0-30$ & 0.87 & 0.67 & 0.46 & 0.25 \\
\cline { 3 - 6 } & & & & &
\end{tabular}

$\underline{\text { Defoliate }}$

Native cover at time $t+1$

\begin{tabular}{cr|c|c|c|c|} 
& & $60-100$ & $45-60$ & $30-45$ & $0-30$ \\
\cline { 3 - 6 } Native & $60-100$ & 0.88 & 0.57 & 0.31 & 0.14 \\
\cline { 3 - 6 } cover at & $45-60$ & 0.91 & 0.70 & 0.45 & 0.23 \\
\cline { 3 - 6 } time $t$ & $30-45$ & 0.92 & 0.74 & 0.54 & 0.30 \\
\cline { 3 - 6 } & $0-30$ & 0.93 & 0.77 & 0.58 & 0.38 \\
\cline { 3 - 6 } & & & & &
\end{tabular}

Burn within window

Native cover at time $t+1$

\begin{tabular}{cr|c|c|c|c|} 
& & \multicolumn{1}{c}{$60-100$} & $45-60$ & $30-45$ & $0-30$ \\
\cline { 3 - 6 } Native & $60-100$ & 0.83 & 0.50 & 0.25 & 0.10 \\
\cline { 3 - 6 } cover at & $45-60$ & 0.86 & 0.64 & 0.37 & 0.16 \\
\cline { 3 - 6 } time $t$ & $30-45$ & 0.88 & 0.67 & 0.46 & 0.22 \\
\cline { 3 - 6 } & $0-30$ & 0.89 & 0.70 & 0.50 & 0.29 \\
\cline { 3 - 6 } & & & & &
\end{tabular}

Here we describe the utility by its constituent parts to demonstrate how it encompasses different aspects of the managers' value system and provides the information necessary to fully express the management objective. We describe the anatomy of a single utility matrix (table $2 A$, Rest) and explain the values of the managers that it captures.

A single matrix is two-dimensional and indexed by the native cover level at time $t$, before a management action has been applied, and the native cover at time $t+1$, after a management action has been applied. Thus, the utility matrix captures a single time step between two consecutive management years.

The utility focuses on native cover of the management unit, and does not take into account the full vegetation state structure of the management unit, which also includes type of dominant invader. The management objective is to increase native cover, regardless of what comprises the non-native vegetation; it is for this reason that the utility focuses solely on amount of native cover. The full vegetation structure, including dominant invader type, is used in the modeling component of the decision framework because invader type affects how the objective can be achieved through management.

The values within the matrix quantify the managers' subjective feelings about each of the possible outcomes or transitions between the beginning native cover level at time $t$ and the resulting 
native cover level at time $t+1$, after the management action has been implemented. The values represent the level of satisfaction with each of the possible outcomes, where 0 is the least satisfied one can be and 1 is the most satisfied one can be.

There are three partitions of the matrix that reflect three distinct aspects of the managers' value system; these partitions include (1) along the diagonal, (2) below the diagonal, and (3) above the diagonal. Below we describe how the utility values contained within each of these three partitions capture three important aspects of managers' value system.

Along the Diagonal. - Values along the diagonal represent the level of satisfaction felt with maintaining each of the four levels of native cover; that is, starting in a given native cover level and time $t$, applying a management action, and remaining in the same native cover level at time $t+1$. Satisfaction is greatest when maintaining the highest level of native cover and decreases with maintaining lower levels of native cover; this is demonstrated by the decline in utility as one moves from the upper left to the lower right, along the diagonal (table $2 A$, Rest). The pattern of values along the diagonal captures the aspect that managers value higher native cover.

Below the Diagonal.-Values below the diagonal represent the level of satisfaction with gaining native cover and making improvements from lower to higher native cover levels; that is, starting in a given native cover level at time $t$, applying a management action, and gaining native cover such that the management unit transitions to a higher level of native cover at time $t+1$. When looking at these values per row (that is, from the perspective of the starting level of native cover at time $t$ ), the values are higher than the maintenance values on the diagonal because managers are more satisfied when there is a gain in native cover than when the existing native cover is maintained (table $2 A$, Rest). When looking at these below-diagonal values per column (that is, from the perspective of the resulting level of native cover at time $t+1$ ), they are higher than the maintenance values on the diagonal, revealing that even though the resulting native cover is the same, managers are more satisfied with the level of native cover if it resulted from a gain in native cover since time $t$ rather than simply a maintenance of existing native cover. The pattern of values below the diagonal captures the aspect that managers value gaining native cover (that is, improvement of system state).

Above the Diagonal. - Values above the diagonal represent the level of satisfaction with losing native cover and degrading from higher to lower native cover level; that is, starting in a given native cover level at time $t$, applying a management action, and losing native cover such that the management unit transitions to a lower level of native cover at time $t+1$. When looking at these values per row (that is, from the perspective of the starting level of native cover at time $t$ ), the values are lower than the maintenance values on the diagonal because managers are less satisfied when there is a loss in native cover than when the existing native cover is maintained (table $2 A$, Rest). When looking at these abovediagonal values per column (that is, from the perspective of the resulting level of native cover at time $t+1$ ), they are lower than the maintenance values on the diagonal, revealing that even though the resulting native cover is the same, managers are less satisfied with the level of native cover if it resulted from a loss of native cover since time $t$ rather than simply a maintenance of existing native cover. The pattern of values above the diagonal captures the aspect that managers devalue losing native cover (that is, degradation of system state).

The three aspects of managers' values outlined above all express different facets of the first part of the management objective, to increase the cover of native grasses and forbs. These three aspects are all captured in the pattern of the utility values within a single utility matrix. The second part of the management objective, to minimize cost, is expressed by having a separate utility matrix for each alternative management action (table 2); this construction of the utility allows us to recognize cost by accounting for the management action that was taken to prompt the transition between the starting 
native cover level and the future native cover level. The values within each matrix of the alternative management actions follow the same pattern and have the same general internal relationships among values as described for the rest matrix ${ }^{1}$ (table $2 A$, Rest); however, the values are smaller. This overall reduction in the matrix values per alternative management action is relative to the cost of the management action, where rest is the least expensive action one can implement, burn/graze is the most expensive action one can implement, and defoliate, graze, and burn fall in between the two extremes. The specific details of how relative cost was incorporated into the utility values are described in appendix $1 \mathrm{f}$. The reduction in utility value per alternative management action captures how managers' level of satisfaction with having a given level of native cover is reduced or discounted by the cost incurred to achieve it; this is true whether the existing level of native cover was the result of maintenance, improvement, or degradation (that is, the cost aspect is layered on top of the other three aspects). Overall, the full utility captures the essence of the managers' value system - that managers value having higher levels of native cover for the least investment.

\subsubsection{Objective Function}

The utility, which describes the managers' value system, is the annual measure of what the manager receives from the system in return for what he/she invests. Quantifying the utility in this way allows us to state the management objective in a mathematical form, known as the objective function (equation 1). Where $u$ is the utility for transition between system states $S_{t}$ and $S_{t+1}$ (levels of native cover) achieved under management action $a, \lambda$ is a discount factor, and $t$ is time, the objective function maximizes the cumulative expected utility through time, given the system state and the implemented management action at each point in time.

$$
\max _{a_{t+1} \in A} \sum_{t=t_{0}}^{T} \lambda^{t-t_{0}} E\left\{u\left(S_{t}, S_{t+1}, a_{t+1}\right)\right\}
$$

We used an endpoint, $T$, of 1,000 years; this is a sufficiently large value to represent an indefinite time horizon. We seek a recurrent reward (utility) each year over an indefinite time horizon; thus, the objective function implies that we seek long-term conservation of the resource, native cover. The 1,000-year time horizon resulted in a decision policy that was approximately stationary (see section 4.8 Optimization), but could be run within a reasonable amount time (e.g., no longer than 2 days ) given our computing power.

We used a discounting factor, $\lambda$, of 0.993 . The effect of the discount factor is to reduce the value of the utility received in the future relative to the utility received today for the same return. The discount factor can range between 0 and 1, where a discount factor of 1 reflects no discounting. The larger the discount factor (that is, the closer it is to 1), the less the future is discounted. Put into context, our choice of a discount factor of 0.993 translates into the utility gained 100 years in the future discounted by one-half of what would be received for the same event had it taken place today. Specifically, if today $t=t_{0}$ and 100 years from today $t=t_{0}+100$, then $\lambda^{t-t_{0}}$ for today is $0.993^{0}=1$ and $\lambda^{t-t_{0}}$ for 100 years in the future is $0.993^{100}=0.5$. Because we multiply the utility gained by $\lambda^{t-t_{0}}$, the utility gained today is not discounted $\left(\lambda^{t-t_{0}}=1\right)$, whereas the utility gained 100 years in the future is discounted by onehalf $\left(\lambda^{t-t_{0}}=0.5\right)$. We chose to discount future utilities for practical reasons of computational stability and so the optimization procedure (see section 4.8 Optimization) would converge on a stationary policy.

\footnotetext{
${ }^{1}$ But see appendix $1 \mathrm{f}$ for a description of how the relative effect of improving and degrading differs with the cost of alternative management actions.
} 
We believe that our choice of discount factor is sufficiently large such that we do not undervalue the future, which is important when our aim is long-term conservation of native prairie.

We use the objective function, the quantitative description of the management objective as a long-term sum of discounted utilities, in the optimization step (see section 4.8 Optimization) to determine the sequence of management decisions through time that will maximize expected cumulative utility. Because the utility directly reflects the management objective, maximizing expected cumulative utility through time identifies the sequence of decisions through time that is expected to achieve the management objective.

\subsection{Management Alternatives}

We next defined the menu of admissible decision alternatives that managers can use to pursue the management objective. Management decisions are supplied as input to decision models, and different decisions should yield different expected outcomes under the models. Management of grasslands is characterized by considerable uncontrolled natural variability; thus, it is important that differences in outcome among management alternatives be large and distinct if management is to be informative. A decision set containing a few, coarse-grained alternatives is more likely to provide rapid gains in learning than one containing a large number of subtly distinguished options. Therefore, construction of the set of decision alternatives was guided by actions likely to generate the greatest diversity in outcomes, as well as by logistical and political feasibility. Over the course of development of NPAM, we considered two successive prototypes of the decision framework; the second prototype was ultimately adopted as the decision framework for the implementation phase of NPAM.

\subsubsection{Provisional Prototype: Mixed-Grass and Tallgrass Alternative Management Actions}

During the initial workshop, we elicited ideas from the participants about treatment options, focusing on management actions that are traditionally available to cooperators, identified constraints in their use, and narrowed the management alternatives to a manageable number to facilitate learning. The cooperators outlined five alternative management actions: rest, hay, graze, burn, and burn/graze combination. Each of the five management alternatives was generally defined with broad sideboards for timing, repetition, and intensity of application; within these sideboards, specific implementation of the action was left to the discretion of the manager. In each management year (defined as September 1August 31), for each management unit, a manager selects one management action from this menu to apply to the unit (see appendix 2 for an overview of the first provisional prototype).

During the 2010 and 2011 management years (September 2009-August 2010 and September 2010-August 2011, respectively) the five alternative management actions were used for mixed-grass and tallgrass prairie units. After completing the first adaptive management cycle (2011 management year), we modified the menu of alternative management actions and made them grass-type specific (mixed-grass and tallgrass); this modification was based on feedback from the FWS members of the Science Team and cooperators. The modifications per grass type are described next.

\subsubsection{Final Prototype: Alternative Management Actions by Grass Type}

\subsubsection{Mixed-Grass}

We dropped hay as an alternative management action on mixed-grass units and retained the other four alternative actions: rest, graze, burn, and burn/graze. Haying was removed as an alternative management action because there was not a biological argument for using it as a form of defoliation to manage for native grasses and forbs or against the cool-season invasive species, smooth brome and 
Kentucky bluegrass. Lacking a biological basis for its use, we also lacked information for predicting a response to its use with respect to changes in cover of native grasses and forbs and cool-season invasive species. Additionally, cooperators of mixed-grass prairies typically did not use haying as a form of management either for native species or against cool-season invasive species, and they did not express an interest in maintaining haying as an alternative management action. More details of each of the four alternative management actions are below.

Rest-Rest designates the lack of any defoliation treatment (graze or burn) applied to the management unit during the management year. Special, targeted treatments (e.g., spot herbicide treatments or clipping) within the management unit may be implemented and the unit is still considered to have received a rest treatment.

Graze - The graze treatment is defined as the targeted use of grazing ungulates as the single form of defoliation that is implemented during a management year. In general, any timing, duration, intensity (stocking rate), or grazing species that targets the rapid growth phase of cool-season invasive plant species and is considered consistent with best management practices for native prairie ecosystems is permitted under NPAM.

Burn-The burn treatment is defined as the application of fire as the single form of defoliation that is carried out during a management year. In general, any timing, intensity, or method of application that is considered consistent with best management practices for native prairie ecosystems is permitted under NPAM.

Burn/Graze - The burn/graze treatment is defined as the use of the graze and the burn treatments, as defined above, in the course of a single management year. The burn and graze actions may be both applied in the fall, both applied in the spring, or applied in different seasons. Typically, the burn action precedes the graze action, but reversal of these actions can take place and is acceptable. Implementation of the burn and graze actions must be carried out as described above.

Valid combinations of treatments - Within a management year, more than one management action may be carried out on a unit. Multiple grazes, multiple burns, and multiple grazes and burns are all valid NPAM treatments. The type of actions, and not the frequency with which they occurred, is the defining factor. The rules used to classify the action of a given unit within a management year are detailed in the Coordinator Database User Guide (see appendix D of digital appendix 2). The management action classifications per unit apply to all aspects of the decision framework, including the utility (section 4.2.2) and the vegetation models (section 4.5.1).

\subsubsection{Tallgrass}

We modified the alternative management actions for tallgrass prairie units to account for the timing of management relative to the cool-season window (defined below), a phenomenon that is present in tallgrass, but not mixed-grass, prairies. Through discussion with the Science Team members, we arrived at four alternative management actions that are relevant to the way in which tallgrass prairie units are managed to benefit native species of grasses and forbs and to hinder cool-season invasive species; these actions include rest, graze within window, burn within window, and defoliate. The 'within window' descriptor refers to carrying out said action within the fall or spring cool-season window, which is identified per locale each season. 'Defoliate' refers to any type of defoliation action (graze or burn) that is applied outside of the cool-season window or to a haying action that is carried out at any time during the management year (that is, within or outside of the cool-season window). Below are more detailed descriptions of the cool-season window and the four alternative management actions.

Cool-Season Window-The cool-season window is a phenological attribute that is considered only for tallgrass prairie units under NPAM [see the provisional model described by Willson and 
Stubbendieck (2000) for relevance and motivation]. It is the period when cool-season invasive grass species are actively growing and vulnerable to damage via select management actions, but warm-season grass species are not active and are thus less susceptible to damage via the same actions. Two such windows of opportunity come to pass each management year: one in the fall and the other in the spring. Because of the large spatial coverage of the NPAM project, phenological cues, rather than calendar dates, are used to define when the windows begin and end. The dates of occurrence will vary by location and year and so must be determined each fall and spring of each management year at each station.

Fall window ${ }^{2}$ - The phenological cue for the beginning of the fall window is 're-green up' when warm-season grass species have gone to seed, but cool-season invasive grass species have resumed tiller growth after the summer dormant period. The fall window will end after the first killing frost (most of the plants are turning yellow). Typically this window lasts 4-6 weeks and takes place within a period from mid-September to the end of October, depending on the year, weather, and location.

Spring window - The phenological cue for the beginning of the spring window is when smooth brome reaches the five-leaf stage. The spring window closes when smooth brome inflorescences are visible. The spring window captures the rapid growth phase of cool-season invasive plant species while excluding the growth and reproductive stages of native warmseason species that come about later in the season. Typically this window lasts about 30 days and occurs within a period from early May to mid-June, depending on the year, weather, and location.

NPAM definitions of 'within the window' and 'outside of the window' by phenological cues are as follows:

- Fall within window: greater than 50 percent of warm-season native grasses have gone to seed and greater than 25 percent of cool-season invasive grasses are fall green-up plants

- Spring within window: greater than 50 percent of smooth brome has at least five leaves and greater than 50 percent of smooth brome has visible inflorescences

- Fall outside of window: greater than 50 percent of warm-season native grasses are still active (before window) or greater than 75 percent of cool-season invasive grasses have senesced for the season (after window)

- Spring outside of window: greater than 50 percent of smooth brome has fewer than five leaves (before window) or greater than 50 percent of smooth brome inflorescences are visible or have already passed (after window)

Rest - Rest designates the lack of any defoliation treatment (that is, graze within window, burn within window, or defoliate) applied to the management unit during the management year. Special, targeted treatments (e.g., spot herbicide treatments or manual pulling) within the management unit may be implemented and the unit is still considered to have received a rest treatment.

\footnotetext{
${ }^{2}$ In late 2012, members of the NPAM Advisory Team (see section 7.1 NPAM Advisory Team) expressed concern about the phenological cues they had chosen for defining the fall window. Unlike the phenological cues for the spring window, which were based on existing research (Willson and Stubbendieck, 2000), there is no known similar research on which to base the phenological cues of the fall window. The Advisory Team did not feel that the necessary information was available at this time to propose alternative cues to define the window or to determine an alternative method for defining the fall window. For this reason, during the annual processing of the tallgrass data, actions that were applied in the fall (that is, between September 1 and December 31) were considered unclassifiable; that is, we were not able to classify them as taking place 'within window' or 'outside window'. More work is need to determine reliable phenological cues that can be observed by cooperators and used to identify the timing of the fall window (see section 7.2.1 Near-Term).
} 
Graze within window - The graze within window treatment is defined as the use of grazing ungulates within the cool-season window as the single form of defoliation that is applied during a management year. The 'within cool-season window' condition is satisfied if the seasonal specific conditions are met as described below.

\section{Fall window}

- Turning animals out on the unit-Animals may be turned out on the unit within 2 weeks before the start of the window, or at any time after the start of the window.

- Length of the graze-Assure that the graze period within the window is at least 2 weeks long or covers the entire length of the window.

- Removing animals from the unit-Animals may be removed at any time within 2 weeks before the close of the window, or at any time up to 4 weeks after the close of the window.

Spring window

- Turning animals out on the unit-Animals may be turned out on the unit at any time before or after the start of the window.

- Length of the graze-Assure that the graze period within the window is at least 2 weeks long or covers the entire length of the window.

- Removing animals from the unit-Animals may be removed at any time within 1 week before the close of the window, or at any time up to 2 weeks after the close of the window.

Within these side-boards, any timing, duration, intensity (stocking rate), or grazing species that is considered consistent with best management practices for native prairie ecosystems is permitted under NPAM.

Burn within window - The burn within window treatment is defined as the application of fire within the cool-season window as the single form of defoliation that is carried out during a management year. Within this window, any timing, intensity, or method of application that is considered consistent with best management practices for native prairie ecosystems is permitted under NPAM.

Defoliate - The defoliate treatment is any graze or burn action that does not meet the "within cool-season window' criteria outlined above. The defoliate classification also includes haying actions that are applied at any time of the year. For all these actions, any timing, intensity, or method of application that is considered consistent with best management practices for native prairie ecosystems is permitted under NPAM.

Valid combinations of treatments - Within a management year, more than one management action may be carried out on a unit; however, only some combinations of treatments are valid in NPAM. Valid multiple treatments include: multiple grazes within the window, multiple burns within the window, and multiple defoliations (that is, grazes and burns outside of the window, or haying events). Other combinations of treatments (e.g., graze within window and burn within window; graze within window and defoliate; or burn within window and defoliate) are not valid. Data from units with invalid combinations of treatments are not included in model-updating for that year. The type of actions, and not the frequency with which they occurred, is the defining factor. The rules used to classify the action of a given unit within a management year are detailed in the Coordinator Database User Guide (see appendix D of digital appendix 2). The management action classifications per unit apply to all aspects of the decision framework, including the utility (section 4.2.2 Utility) and the vegetation models (section 4.5.1 Vegetation Model). 


\subsection{Sampling and Modeling Approaches for Making Post Hoc Determination of Tallgrass Management Actions}

The NPAM decision framework for tallgrass units is built around the concept that cool-season windows (fall and spring) exist during which grassland management can impact cool-season invasive grasses without negatively impacting native warm season grasses (e.g., Willson and Stubbendieck, 2000). Timing and duration of the management event relative to the cool-season window is required to classify the action as taking place within the window or outside of the window. Neither the fall nor spring cool-season window is static; start and end periods for fall and spring windows likely differ among years and locations. For this reason, phenological data must be collected annually.

Phenology data are collected at two spatial scales: (1) the management-unit scale and (2) the station-level scale. These are two related but different data collection efforts. Management unit-level phenological data are collected at the specific locations of each tallgrass unit at specific points in timethe start and end dates of a management action. Station-level phenological data are collected on a repeated three-day cycle during the fall and spring seasons at select, representative locations that span the tallgrass region. Locations are not fixed and can vary from year to year, depending on logistical and environmental constraints, but it is important that data are acquired at widely spaced locations across the region; figure 3 shows the location of the sites monitored during 2011. Station-level data are used to track the progression of phenology, and thus identify the start and end of the fall and spring cool-season windows, across the tallgrass region. We use these station-level phenological data as input data points to an isopleth model that outputs a smoothed, interpolated surface of fall and spring cool-season window start and end dates across the tallgrass region of the sampled area (see digital appendix 3 for details). From the interpolated surface, approximate window start and end dates can be extracted for the specific location of individual tallgrass units. We use the management-unit level data and isopleth model predictions individually or jointly to classify management actions as taking place within or outside of the cool-season windows [see appendixes B and C within the Coordinator Database User Guide (digital appendix 2) for the specific rules of classification and level of phenological data used]. Because of the need to collect consistent and accurate data on phenology, a Phenology User Guide was created as a stand-alone reference for NPAM cooperators who manage tallgrass units (digital appendix 4); details of the specific protocols for collecting phenological data are found in this user guide. 


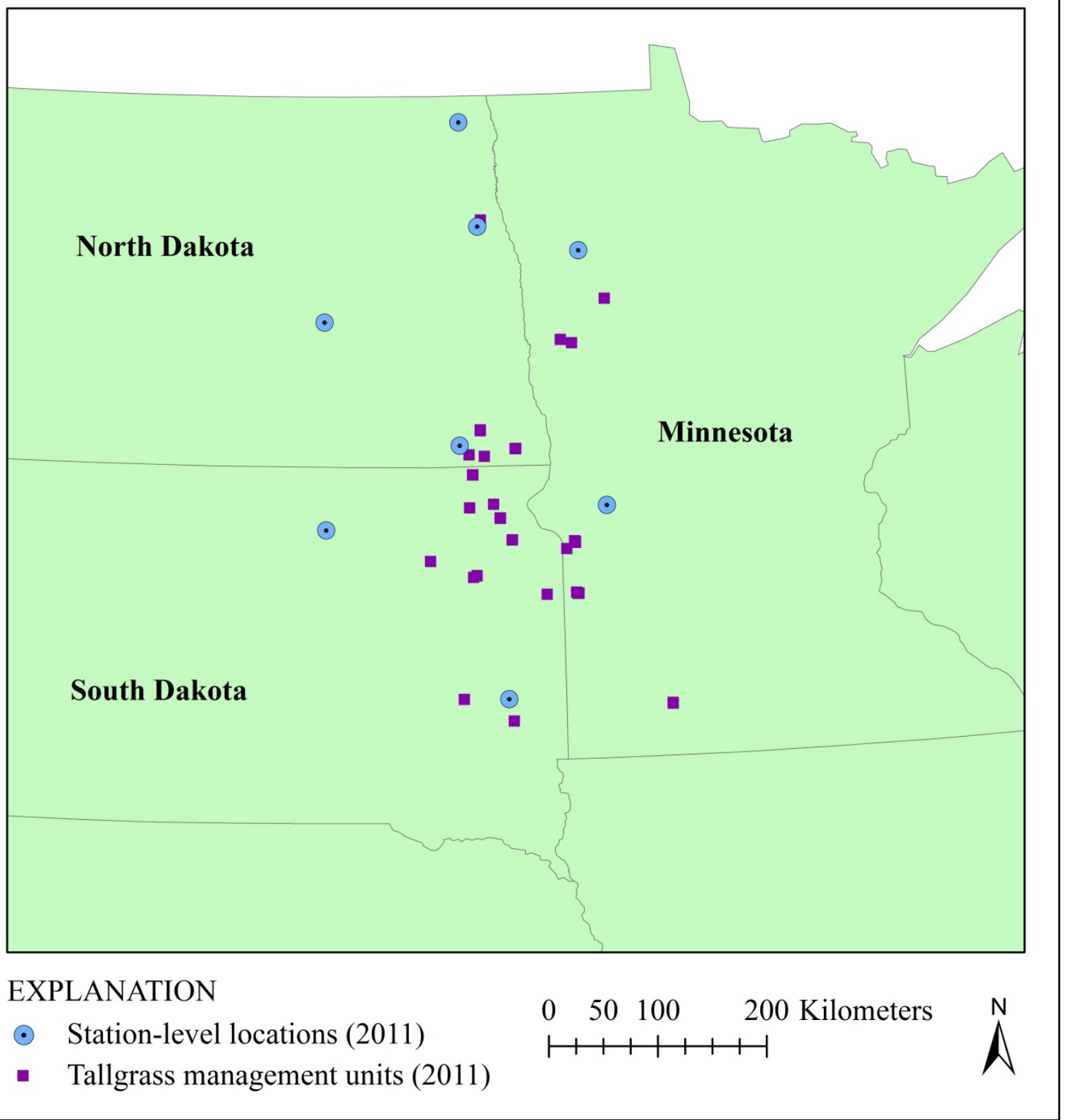

Figure 3. Location of the station-level sites monitored for phenology during 2011 and the tallgrass management units enrolled in the Native Prairie Adaptive Management initiative in 2011.

\subsection{Representation of the System State}

\subsubsection{Vegetation State}

We define the state of the vegetation on each management unit at a particular time by two characteristics: the amount of cover of native grasses and forbs and the type of invasive grass that is dominant. We recognize four discrete states of native prairie cover: 0-30 percent, 30-45 percent, 45-60 
percent, and 60-100 percent $^{3}$. Within each of the four states of native prairie cover, we recognize the dominant invasive as smooth brome, Kentucky bluegrass, co-dominant smooth brome and Kentucky bluegrass, or some other non-native species. The four states of native prairie cover in combination with the four states of dominant invasive, result in 16 discrete possible vegetation states (table 3 ).

Table 3. Vegetation states recognized in Native Prairie Adaptive Management

[The vegetation of each management unit is categorized into one of 16 discrete states, depending on its percent cover of native grasses and forbs (0-30, 30-45, 45-60, and 60-100) and its dominant invasive [smooth brome (SB), smooth brome/Kentucky bluegrass co-dominant (CO), Kentucky bluegrass (KB), and remainder (RM)]. We define dominance as functions of cover amounts of $\mathrm{SB}, \mathrm{KB}$, and $\mathrm{RM}$ as follows: remainder dominant if $\mathrm{RM} /(\mathrm{SB}+\mathrm{KB}+\mathrm{RM})>=0.67$; else smooth brome dominant if $\mathrm{SB} /(\mathrm{SB}+\mathrm{KB})>=0.67$; Kentucky bluegrass dominant if $\mathrm{KB} /(\mathrm{SB}+\mathrm{KB})>=0.67$; or co-dominant if none of the aforementioned conditions are met]

Dominant invasive

\begin{tabular}{cc|c|c|c|c|} 
& & \multicolumn{2}{c}{ SB } & \multicolumn{1}{c}{ CO } & \multicolumn{2}{c}{ KB } & \multicolumn{1}{c}{ RM } \\
\cline { 3 - 6 } & $60-100 \%$ & 1 & 2 & 3 & 4 \\
\cline { 3 - 6 } Native & $45-60 \%$ & 5 & 6 & 7 & 8 \\
\cline { 2 - 6 } cover & $30-45 \%$ & 9 & 10 & 11 & 12 \\
\cline { 2 - 6 } & $0-30 \%$ & 13 & 14 & 15 & 16 \\
\cline { 3 - 6 } & & & & &
\end{tabular}

\subsubsection{Defoliation State}

In addition to the vegetation state of a management unit, we defined the defoliation history of a unit over a seven-year window of previous management. The defoliation state is a combination of two characteristics of the management unit: the number of years since the last defoliation event (any NPAM management action other than rest) took place and the defoliation index. Descriptions of these two characteristics, and how we combine them to arrive at the defoliation state, are covered below.

\subsubsection{Years Since Last Defoliation}

The years since defoliation captures the length of time (in years) since the unit received a NPAM management action other than rest. Years since defoliation is determined from the perspective of the beginning (September 1) of the present management year, $t$. A unit that received one or more active forms of management (that is, an action other than rest) in the previous management year, $t$ - 1 , is considered to have been defoliated 1 year ago, whereas a unit that was rested during all 7 years is considered to have been defoliated more than 7 years ago (table 4 ). We classified the years since defoliation into three categories: 1 year ago, 2-4 years ago, and 5 or more years ago.

\footnotetext{
${ }^{3}$ The division of the native prairie cover into the four levels was determined by an elicitation exercise with the FWS members of the Science Team. A complete overview of the exercise, results, and final determination of the native cover levels can be found in appendixes $1 \mathrm{a}, 1 \mathrm{~b}$, and $1 \mathrm{c}$, respectively.
} 
Table 4. Years since last defoliation over a seven-year window of past management history.

[There are eight cases; each row represents a single case. Where $t$ is the current year and $t-1$ is the most recent year in which management was applied, $t-1$ to $t-7$ represent the seven-year window of past management history. Years that the management unit was rested are indicated by a ' 0 '. Years that the management unit was treated with at least one non-rest management action are indicted by a ' 1 ' and denote a defoliation event. Shaded cells may be a ' 0 ' or a ' 1 ', as their status does not change the years since defoliation classification of the unit. The actual number of years since last defoliation is classified into one of three categories: 1 year ago, 2-4 years ago, and 5 or more years ago]

\begin{tabular}{|c|c|c|c|c|c|c|c|c|}
\hline \multicolumn{7}{|c|}{ Seven-year window } & \multirow{2}{*}{$\begin{array}{c}\text { Years } \\
\text { since } \\
\text { (actual) }\end{array}$} & \multirow{2}{*}{$\begin{array}{c}\text { Years } \\
\text { since } \\
\text { (classified) }\end{array}$} \\
\hline$t-1$ & $t-2$ & $t-3$ & $t-4$ & $t-5$ & $t-6$ & $t-7$ & & \\
\hline 1 & & & & & & & 1 & 1 \\
\hline 0 & 1 & & & & & & 2 & $2-4$ \\
\hline 0 & 0 & 1 & & & & & 3 & $2-4$ \\
\hline 0 & 0 & 0 & 1 & & & & 4 & $2-4$ \\
\hline 0 & 0 & 0 & 0 & 1 & & & 5 & $5+$ \\
\hline 0 & 0 & 0 & 0 & 0 & 1 & & 6 & $5+$ \\
\hline 0 & 0 & 0 & 0 & 0 & 0 & 1 & 7 & $5+$ \\
\hline 0 & 0 & 0 & 0 & 0 & 0 & 0 & $>7$ & $5+$ \\
\hline
\end{tabular}

\subsubsection{Defoliation Index}

The defoliation index (equation 2) takes into account the frequency of defoliation events during the seven-year window (that is, the number of management years within the seven-year window in which at least one defoliation event occurred) and the number of years since the last defoliation. Where $D=$ defoliation index, $w=$ weight (fig.4; table 5), $k=$ seven-year window, $i=$ window year, and $d=$ defoliation event ( 0 or 1$)$, the equation for calculating the defoliation index is as follows

$D=w \sum_{i}^{k} d_{i}$

The index is simply a means of assigning an 'importance of recentness' weight to the number of defoliation actions executed during the seven-year window. We used a declining S-shaped curve to determine the weighting scheme over the seven-year window, where the rate of decline is slow at first, and falls off more rapidly with passing time (fig. 4; table 5). 


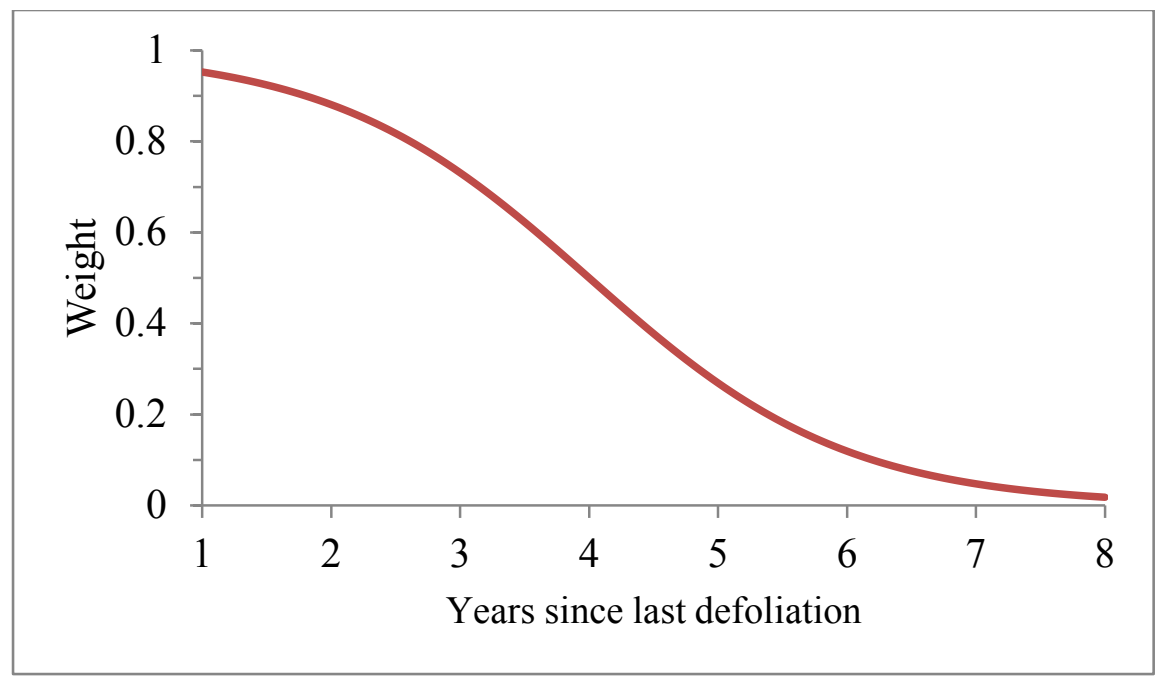

Figure 4. Declining S-shaped logistic curve used to determine the weight for calculating the defoliation index.

Table 5. The weights used in calculating the defoliation index, derived from the declining S-shaped curve (fig. 4).

\begin{tabular}{lcccccccc}
\hline Years since last defoliation & 1 & 2 & 3 & 4 & 5 & 6 & 7 & $>7$ \\
\hline Weight & 0.953 & 0.881 & 0.731 & 0.500 & 0.269 & 0.119 & 0.047 & 0.018 \\
\hline
\end{tabular}

All combinations of defoliation/non-defoliation actions within the seven-year window yield 128 different defoliation scenarios that can potentially result (table 6 ). We divided the full range of defoliation indices into three defoliation levels: low $(D<2)$, medium $(2 \leq D<4)$, and high $(D>4)$. We examined how the selection of these boundary points divided the 128 possible past management history scenarios among the possible combinations of 'years since last defoliation' and 'total number of defoliations' (table 7) and confirmed with our FWS Science Team partners that the divisions were consistent with their thinking regarding the biological meaning of the defoliation levels in the context of these two characteristics.

As an additional check of the appropriateness of the defoliation level boundary points we chose, we determined the transition probabilities among the three defoliation levels in a one-year time step, given the implementation of rest or some form of defoliation management action within that time step (table 8). For each of the 128 management history scenarios, we determined the resulting management history, defoliation index, and defoliation level at time $t+1$ for the case of (1) implementing rest in the current year or (2) implementing an action other than rest (that is, some form of defoliation). We crosstabulated the results to compute frequencies of defoliation level at time $t$ and defoliation level at time $t+1$ for each of the two forms of treatment. From these frequencies, we calculated the probabilities of transitioning from each of the three defoliation levels at time $t$ to each of the three defoliation levels at time $t^{+1}$ (that is, a one-year time step), given the unit received a rest (no action) and given the unit received some type of defoliation action during that time step (table 8). The transition probabilities exhibit reasonable behavior; extreme jumps between high and low levels do not occur and transitions among adjacent levels are consistent with the relative number of scenarios per defoliation level. Given this behavior, we feel confident that our choice of defoliation level boundary points are suitable. 
Table 6. Defoliation indices for the 128 potential management histories over the seven-year window.

[Year since last defoliation is the number of years since the management unit was treated with at least one non-rest management action (see table 4). Number of defoliations refers to the number of management years in which at least one non-rest management action was applied. Defoliation index is calculated as shown in equation 2. Years ago indicates, for each year of the seven-year window, whether the unit was rested (' 0 ') or received at least one non-rest management action (' 1 '). The table is sorted from high to low defoliation index. The break-points between the three defoliation levels (high, medium, low) are denoted by a dashed line]

\begin{tabular}{cccccccccc}
\hline $\begin{array}{c}\text { Year since } \\
\text { last } \\
\text { defoliation }\end{array}$ & \multirow{2}{*}{ Number of } & defoliations & Defoliation & \multicolumn{7}{c}{ Years ago } \\
\cline { 6 - 10 } & index & 1 & 2 & 3 & 4 & 5 & 6 & 7 \\
\hline 1 & 7 & 6.67 & 1 & 1 & 1 & 1 & 1 & 1 & 1 \\
1 & 6 & 5.72 & 1 & 1 & 1 & 1 & 1 & 1 & 0 \\
1 & 6 & 5.72 & 1 & 1 & 1 & 1 & 1 & 0 & 1 \\
1 & 6 & 5.72 & 1 & 1 & 1 & 1 & 0 & 1 & 1 \\
1 & 6 & 5.72 & 1 & 1 & 1 & 0 & 1 & 1 & 1 \\
1 & 6 & 5.72 & 1 & 1 & 0 & 1 & 1 & 1 & 1 \\
1 & 6 & 5.72 & 1 & 0 & 1 & 1 & 1 & 1 & 1 \\
2 & 6 & 5.28 & 0 & 1 & 1 & 1 & 1 & 1 & 1 \\
1 & 5 & 4.76 & 1 & 1 & 1 & 1 & 1 & 0 & 0 \\
1 & 5 & 4.76 & 1 & 1 & 1 & 1 & 0 & 1 & 0 \\
1 & 5 & 4.76 & 1 & 1 & 1 & 1 & 0 & 0 & 1 \\
1 & 5 & 4.76 & 1 & 1 & 1 & 0 & 1 & 1 & 0 \\
1 & 5 & 4.76 & 1 & 1 & 1 & 0 & 1 & 0 & 1 \\
1 & 5 & 4.76 & 1 & 1 & 0 & 1 & 1 & 1 & 0 \\
1 & 5 & 4.76 & 1 & 1 & 1 & 0 & 0 & 1 & 1 \\
1 & 5 & 4.76 & 1 & 1 & 0 & 1 & 1 & 0 & 1 \\
1 & 5 & 4.76 & 1 & 0 & 1 & 1 & 1 & 1 & 0 \\
1 & 5 & 4.76 & 1 & 0 & 1 & 1 & 1 & 0 & 1 \\
1 & 5 & 4.76 & 1 & 1 & 0 & 1 & 0 & 1 & 1 \\
1 & 5 & 4.76 & 1 & 0 & 1 & 1 & 0 & 1 & 1 \\
1 & 5 & 4.76 & 1 & 1 & 0 & 0 & 1 & 1 & 1 \\
1 & 5 & 4.76 & 1 & 0 & 1 & 0 & 1 & 1 & 1 \\
1 & 5 & 4.76 & 1 & 0 & 0 & 1 & 1 & 1 & 1 \\
2 & 5 & 4.4 & 0 & 1 & 1 & 1 & 1 & 1 & 0 \\
2 & 5 & 4.4 & 0 & 1 & 1 & 1 & 1 & 0 & 1 \\
2 & 5 & 4.4 & 0 & 1 & 1 & 1 & 0 & 1 & 1 \\
2 & 5 & 4.4 & 0 & 1 & 1 & 0 & 1 & 1 & 1 \\
2 & 5 & 4.4 & 0 & 1 & 0 & 1 & 1 & 1 & 1 \\
\hline 1 & 4 & 3.81 & 1 & 1 & 1 & 1 & 0 & 0 & 0 \\
1 & 4 & 3.81 & 1 & 1 & 1 & 0 & 1 & 0 & 0 \\
1 & 4 & 3.81 & 1 & 1 & 1 & 0 & 0 & 1 & 0 \\
1 & 4 & 3.81 & 1 & 1 & 0 & 1 & 1 & 0 & 0
\end{tabular}




\begin{tabular}{|c|c|c|c|c|c|c|c|c|c|}
\hline \multirow{2}{*}{$\begin{array}{c}\text { Year since } \\
\text { last } \\
\text { defoliation }\end{array}$} & \multirow{2}{*}{$\begin{array}{l}\text { Number of } \\
\text { defoliations }\end{array}$} & \multirow{2}{*}{$\begin{array}{c}\text { Defoliation } \\
\text { index } \\
\end{array}$} & \multicolumn{7}{|c|}{ Years ago } \\
\hline & & & 1 & 2 & 3 & 4 & 5 & 6 & 7 \\
\hline 1 & 4 & 3.81 & 1 & 1 & 1 & 0 & 0 & 0 & 1 \\
\hline 1 & 4 & 3.81 & 1 & 0 & 1 & 1 & 1 & 0 & 0 \\
\hline 1 & 4 & 3.81 & 1 & 1 & 0 & 1 & 0 & 1 & 0 \\
\hline 1 & 4 & 3.81 & 1 & 1 & 0 & 1 & 0 & 0 & 1 \\
\hline 1 & 4 & 3.81 & 1 & 0 & 1 & 1 & 0 & 1 & 0 \\
\hline 1 & 4 & 3.81 & 1 & 0 & 1 & 1 & 0 & 0 & 1 \\
\hline 1 & 4 & 3.81 & 1 & 1 & 0 & 0 & 1 & 1 & 0 \\
\hline 1 & 4 & 3.81 & 1 & 1 & 0 & 0 & 1 & 0 & 1 \\
\hline 1 & 4 & 3.81 & 1 & 0 & 1 & 0 & 1 & 1 & 0 \\
\hline 1 & 4 & 3.81 & 1 & 0 & 1 & 0 & 1 & 0 & 1 \\
\hline 1 & 4 & 3.81 & 1 & 1 & 0 & 0 & 0 & 1 & 1 \\
\hline 1 & 4 & 3.81 & 1 & 0 & 0 & 1 & 1 & 1 & 0 \\
\hline 1 & 4 & 3.81 & 1 & 0 & 1 & 0 & 0 & 1 & 1 \\
\hline 1 & 4 & 3.81 & 1 & 0 & 0 & 1 & 1 & 0 & 1 \\
\hline 1 & 4 & 3.81 & 1 & 0 & 0 & 1 & 0 & 1 & 1 \\
\hline 1 & 4 & 3.81 & 1 & 0 & 0 & 0 & 1 & 1 & 1 \\
\hline 3 & 5 & 3.66 & 0 & 0 & 1 & 1 & 1 & 1 & 1 \\
\hline 2 & 4 & 3.52 & 0 & 1 & 1 & 1 & 1 & 0 & 0 \\
\hline 2 & 4 & 3.52 & 0 & 1 & 1 & 1 & 0 & 1 & 0 \\
\hline 2 & 4 & 3.52 & 0 & 1 & 1 & 1 & 0 & 0 & 1 \\
\hline 2 & 4 & 3.52 & 0 & 1 & 1 & 0 & 1 & 1 & 0 \\
\hline 2 & 4 & 3.52 & 0 & 1 & 1 & 0 & 1 & 0 & 1 \\
\hline 2 & 4 & 3.52 & 0 & 1 & 0 & 1 & 1 & 1 & 0 \\
\hline 2 & 4 & 3.52 & 0 & 1 & 1 & 0 & 0 & 1 & 1 \\
\hline 2 & 4 & 3.52 & 0 & 1 & 0 & 1 & 1 & 0 & 1 \\
\hline 2 & 4 & 3.52 & 0 & 1 & 0 & 1 & 0 & 1 & 1 \\
\hline 2 & 4 & 3.52 & 0 & 1 & 0 & 0 & 1 & 1 & 1 \\
\hline 3 & 4 & 2.92 & 0 & 0 & 1 & 1 & 1 & 1 & 0 \\
\hline 3 & 4 & 2.92 & 0 & 0 & 1 & 1 & 1 & 0 & 1 \\
\hline 3 & 4 & 2.92 & 0 & 0 & 1 & 1 & 0 & 1 & 1 \\
\hline 3 & 4 & 2.92 & 0 & 0 & 1 & 0 & 1 & 1 & 1 \\
\hline 1 & 3 & 2.86 & 1 & 1 & 1 & 0 & 0 & 0 & 0 \\
\hline 1 & 3 & 2.86 & 1 & 1 & 0 & 1 & 0 & 0 & 0 \\
\hline 1 & 3 & 2.86 & 1 & 0 & 1 & 1 & 0 & 0 & 0 \\
\hline 1 & 3 & 2.86 & 1 & 1 & 0 & 0 & 1 & 0 & 0 \\
\hline 1 & 3 & 2.86 & 1 & 0 & 1 & 0 & 1 & 0 & 0 \\
\hline 1 & 3 & 2.86 & 1 & 1 & 0 & 0 & 0 & 1 & 0 \\
\hline 1 & 3 & 2.86 & 1 & 1 & 0 & 0 & 0 & 0 & 1 \\
\hline 1 & 3 & 2.86 & 1 & 0 & 1 & 0 & 0 & 1 & 0 \\
\hline 1 & 3 & 2.86 & 1 & 0 & 0 & 1 & 1 & 0 & 0 \\
\hline
\end{tabular}




\begin{tabular}{|c|c|c|c|c|c|c|c|c|c|}
\hline \multirow{2}{*}{$\begin{array}{l}\text { Year since } \\
\text { last } \\
\text { defoliation }\end{array}$} & \multirow{2}{*}{$\begin{array}{l}\text { Number of } \\
\text { defoliations }\end{array}$} & \multirow{2}{*}{$\begin{array}{c}\text { Defoliation } \\
\text { index }\end{array}$} & \multicolumn{7}{|c|}{ Years ago } \\
\hline & & & 1 & 2 & 3 & 4 & 5 & 6 & 7 \\
\hline 1 & 3 & 2.86 & 1 & 0 & 1 & 0 & 0 & 0 & 1 \\
\hline 1 & 3 & 2.86 & 1 & 0 & 0 & 1 & 0 & 1 & 0 \\
\hline 1 & 3 & 2.86 & 1 & 0 & 0 & 1 & 0 & 0 & 1 \\
\hline 1 & 3 & 2.86 & 1 & 0 & 0 & 0 & 1 & 1 & 0 \\
\hline 1 & 3 & 2.86 & 1 & 0 & 0 & 0 & 1 & 0 & 1 \\
\hline 1 & 3 & 2.86 & 1 & 0 & 0 & 0 & 0 & 1 & 1 \\
\hline 2 & 3 & 2.64 & 0 & 1 & 1 & 1 & 0 & 0 & 0 \\
\hline 2 & 3 & 2.64 & 0 & 1 & 1 & 0 & 1 & 0 & 0 \\
\hline 2 & 3 & 2.64 & 0 & 1 & 1 & 0 & 0 & 1 & 0 \\
\hline 2 & 3 & 2.64 & 0 & 1 & 0 & 1 & 1 & 0 & 0 \\
\hline 2 & 3 & 2.64 & 0 & 1 & 1 & 0 & 0 & 0 & 1 \\
\hline 2 & 3 & 2.64 & 0 & 1 & 0 & 1 & 0 & 1 & 0 \\
\hline 2 & 3 & 2.64 & 0 & 1 & 0 & 1 & 0 & 0 & 1 \\
\hline 2 & 3 & 2.64 & 0 & 1 & 0 & 0 & 1 & 1 & 0 \\
\hline 2 & 3 & 2.64 & 0 & 1 & 0 & 0 & 1 & 0 & 1 \\
\hline 2 & 3 & 2.64 & 0 & 1 & 0 & 0 & 0 & 1 & 1 \\
\hline 3 & 3 & 2.19 & 0 & 0 & 1 & 1 & 1 & 0 & 0 \\
\hline 3 & 3 & 2.19 & 0 & 0 & 1 & 1 & 0 & 1 & 0 \\
\hline 3 & 3 & 2.19 & 0 & 0 & 1 & 1 & 0 & 0 & 1 \\
\hline 3 & 3 & 2.19 & 0 & 0 & 1 & 0 & 1 & 1 & 0 \\
\hline 3 & 3 & 2.19 & 0 & 0 & 1 & 0 & 1 & 0 & 1 \\
\hline 3 & 3 & 2.19 & 0 & 0 & 1 & 0 & 0 & 1 & 1 \\
\hline 4 & 4 & 2 & 0 & 0 & 0 & 1 & 1 & 1 & 1 \\
\hline 1 & 2 & 1.91 & 1 & 1 & 0 & 0 & 0 & 0 & 0 \\
\hline 1 & 2 & 1.91 & 1 & 0 & 1 & 0 & 0 & 0 & 0 \\
\hline 1 & 2 & 1.91 & 1 & 0 & 0 & 1 & 0 & 0 & 0 \\
\hline 1 & 2 & 1.91 & 1 & 0 & 0 & 0 & 1 & 0 & 0 \\
\hline 1 & 2 & 1.91 & 1 & 0 & 0 & 0 & 0 & 1 & 0 \\
\hline 1 & 2 & 1.91 & 1 & 0 & 0 & 0 & 0 & 0 & 1 \\
\hline 2 & 2 & 1.76 & 0 & 1 & 1 & 0 & 0 & 0 & 0 \\
\hline 2 & 2 & 1.76 & 0 & 1 & 0 & 1 & 0 & 0 & 0 \\
\hline 2 & 2 & 1.76 & 0 & 1 & 0 & 0 & 1 & 0 & 0 \\
\hline 2 & 2 & 1.76 & 0 & 1 & 0 & 0 & 0 & 1 & 0 \\
\hline 2 & 2 & 1.76 & 0 & 1 & 0 & 0 & 0 & 0 & 1 \\
\hline 4 & 3 & 1.5 & 0 & 0 & 0 & 1 & 1 & 1 & 0 \\
\hline 4 & 3 & 1.5 & 0 & 0 & 0 & 1 & 1 & 0 & 1 \\
\hline 4 & 3 & 1.5 & 0 & 0 & 0 & 1 & 0 & 1 & 1 \\
\hline 3 & 2 & 1.46 & 0 & 0 & 1 & 1 & 0 & 0 & 0 \\
\hline 3 & 2 & 1.46 & 0 & 0 & 1 & 0 & 1 & 0 & 0 \\
\hline 3 & 2 & 1.46 & 0 & 0 & 1 & 0 & 0 & 1 & 0 \\
\hline
\end{tabular}




\begin{tabular}{|c|c|c|c|c|c|c|c|c|c|}
\hline \multirow{2}{*}{$\begin{array}{c}\text { Year since } \\
\text { last } \\
\text { defoliation }\end{array}$} & \multirow{2}{*}{$\begin{array}{l}\text { Number of } \\
\text { defoliations }\end{array}$} & \multirow{2}{*}{$\begin{array}{c}\text { Defoliation } \\
\text { index } \\
\end{array}$} & \multicolumn{7}{|c|}{ Years ago } \\
\hline & & & 1 & 2 & 3 & 4 & 5 & 6 & 7 \\
\hline 3 & 2 & 1.46 & 0 & 0 & 1 & 0 & 0 & 0 & 1 \\
\hline 4 & 2 & 1 & 0 & 0 & 0 & 1 & 1 & 0 & 0 \\
\hline 4 & 2 & 1 & 0 & 0 & 0 & 1 & 0 & 1 & 0 \\
\hline 4 & 2 & 1 & 0 & 0 & 0 & 1 & 0 & 0 & 1 \\
\hline 1 & 1 & 0.95 & 1 & 0 & 0 & 0 & 0 & 0 & 0 \\
\hline 2 & 1 & 0.88 & 0 & 1 & 0 & 0 & 0 & 0 & 0 \\
\hline 5 & 3 & 0.81 & 0 & 0 & 0 & 0 & 1 & 1 & 1 \\
\hline 3 & 1 & 0.73 & 0 & 0 & 1 & 0 & 0 & 0 & 0 \\
\hline 5 & 2 & 0.54 & 0 & 0 & 0 & 0 & 1 & 1 & 0 \\
\hline 5 & 2 & 0.54 & 0 & 0 & 0 & 0 & 1 & 0 & 1 \\
\hline 4 & 1 & 0.5 & 0 & 0 & 0 & 1 & 0 & 0 & 0 \\
\hline 5 & 1 & 0.27 & 0 & 0 & 0 & 0 & 1 & 0 & 0 \\
\hline 6 & 2 & 0.24 & 0 & 0 & 0 & 0 & 0 & 1 & 1 \\
\hline 6 & 1 & 0.12 & 0 & 0 & 0 & 0 & 0 & 1 & 0 \\
\hline 7 & 1 & 0.05 & 0 & 0 & 0 & 0 & 0 & 0 & 1 \\
\hline 8 & 0 & 0 & 0 & 0 & 0 & 0 & 0 & 0 & 0 \\
\hline
\end{tabular}

Table 7. Distribution of the 128 past management history scenarios (table 6) among the possible combinations of 'years since last defoliation' by 'number of defoliations'.

[Values in cells represent frequency of scenarios. Coloration represents the defoliation level classification as high, medium, or low]

\begin{tabular}{|c|c|c|c|c|c|c|c|c|}
\hline \multirow{2}{*}{$\begin{array}{c}\text { Years since last } \\
\text { defoliation }\end{array}$} & \multicolumn{8}{|c|}{ Number of defoliations in seven years } \\
\hline & 0 & 1 & 2 & 3 & 4 & 5 & 6 & 7 \\
\hline 1 & & 1 & 6 & 15 & 20 & 15 & 6 & 1 \\
\hline 2 & & 1 & 5 & 10 & 10 & 5 & 1 & \\
\hline 3 & & 1 & 4 & 6 & 4 & 1 & & \\
\hline 4 & & 1 & 3 & 3 & 1 & \multirow{2}{*}{\multicolumn{3}{|c|}{ Frequency by level }} \\
\hline 5 & & 1 & 2 & 1 & & & & \\
\hline 6 & & 1 & 1 & & & & High & 28 \\
\hline 7 & & 1 & & & & & Tedium & 67 \\
\hline$>7$ & 1 & & & & & & Low & 33 \\
\hline
\end{tabular}


Table 8. Probabilities of transitioning among the three defoliation levels in a one-year time step, given the choice of either rest or some form of defoliation as the management action within that time step.

\begin{tabular}{lrrr|rrr}
\hline & \multicolumn{3}{c|}{ Treatment: rest } & \multicolumn{2}{c}{ Treatment: Some form of defoliation } \\
\cline { 2 - 6 } $\begin{array}{l}\text { Starting } \\
\text { level }\end{array}$ & \multicolumn{2}{c|}{ Probability of transition into level } & \multicolumn{2}{c}{ Probability of transition into level } \\
\hline High & High & Medium & Low & High & Medium & Low \\
Medium & 0.43 & 0.57 & 0 & 1 & 0 & 0 \\
Low & 0 & 0.72 & 0.28 & 0.24 & 0.76 & 0 \\
\hline
\end{tabular}

\subsubsection{Combination of Classified Years Since Defoliation and Defoliation Index Level}

The defoliation state is a combination of the time since the last defoliation action occurred, which is broken into classes of 1 year ago, 2-4 years ago, and 5 or more years ago, and the level of defoliation, which is expressed by the defoliation index levels of low, medium, and high. The result, when we combine these two variables, is seven discrete possible defoliation states (table 9).

Table 9. A combination of the years since last defoliation and the defoliation index level results in seven discrete defoliation states.

[At any given time, each management unit is assigned into one of the seven defoliation states based on its defoliation history for the previous 7 years of management. Note that it is possible for units to have low, medium, or high defoliation index levels when they have received a defoliation action within the past 4 years; however, units that have not received a defoliation action in the past 5 or more years are restricted to low defoliation index levels (table 4)]

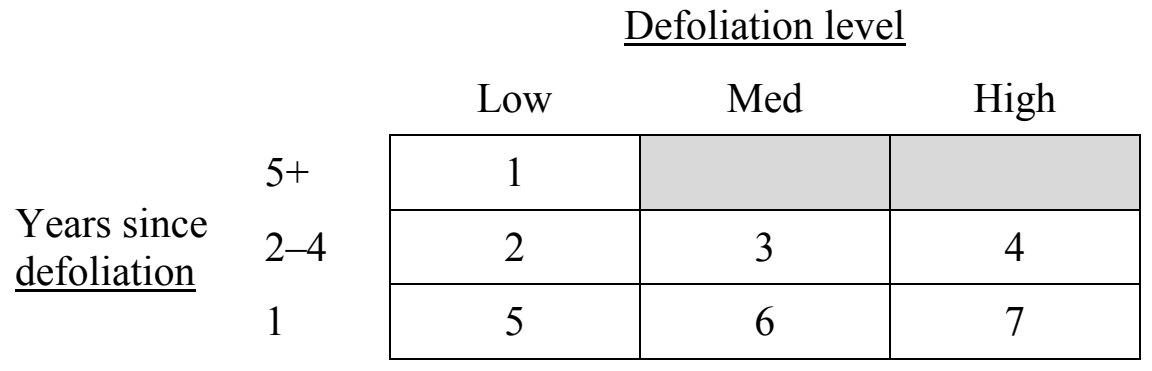

The three-classification level 'years since last defoliation' is a standalone characteristic of the defoliation state space, whereas the actual years since last defoliation is integrated in the defoliation level characteristic (that is, it is used in the calculation of the defoliation index; equation 2; fig. 4; table 5). The second appearance of the actual years since last defoliation in the classified 'years since last defoliation' was necessary for us to account for two additional aspects beyond the effect of defoliation level on vegetation response to management actions; these two aspects include: (1) an alternative hypothesis concerning the vegetation response to a five-year cycle of defoliation (see section 4.6.2 Alternative Models) and (2) the feasibility of carrying out certain management actions in consecutive years (see section 4.7 Partial Controllability). 


\subsubsection{Full System State Structure}

The full system state structure is represented by the combination of the 16 discrete vegetation states (table 3) with the seven discrete defoliation states (table 9), resulting in 112 possible states. At any point in time, a management unit is classified into one of the 112 system states. This classification is determined by four characteristics of the unit: (1) level of native cover, (2) type of dominant invasive, (3) classified number of years since the unit was last defoliated, and (4) defoliation index level.

\subsection{State Transition Probability Model}

\subsubsection{Vegetation Model}

\subsubsection{General Structure}

We use a Markov state transition probability model (Puterman, 1994; Williams and others, 2002) to describe how we think the system behaves in response to management (fig. 5). A $16 \times 16$ transition matrix describes the probability of transitioning from each of the 16 vegetation states at time $t$ to each of the 16 vegetation states at time $t+1$, given a particular management action is applied (fig. 6). Because management actions are likely to affect transition probabilities from one vegetation state to another, and because past management history may affect transition probability from one vegetation state to another, a complete model consists of twelve $16 \times 16$ matrices, one for each of the four alternative management actions (e.g., rest, graze, burn, burn/graze) at each of the three levels of past management defoliation (low, medium, high). Given the current vegetation state of the management unit, the current defoliation level of the management unit, and the management action applied, the model provides a probabilistic prediction of the vegetation state of the unit in the next time-step. A simplistic representation of a complete model is depicted in figure 7; actual models, populated with transition probabilities, are located in digital appendix 6a (mixed-grass) and digital appendix $6 \mathrm{~b}$ (tallgrass). 


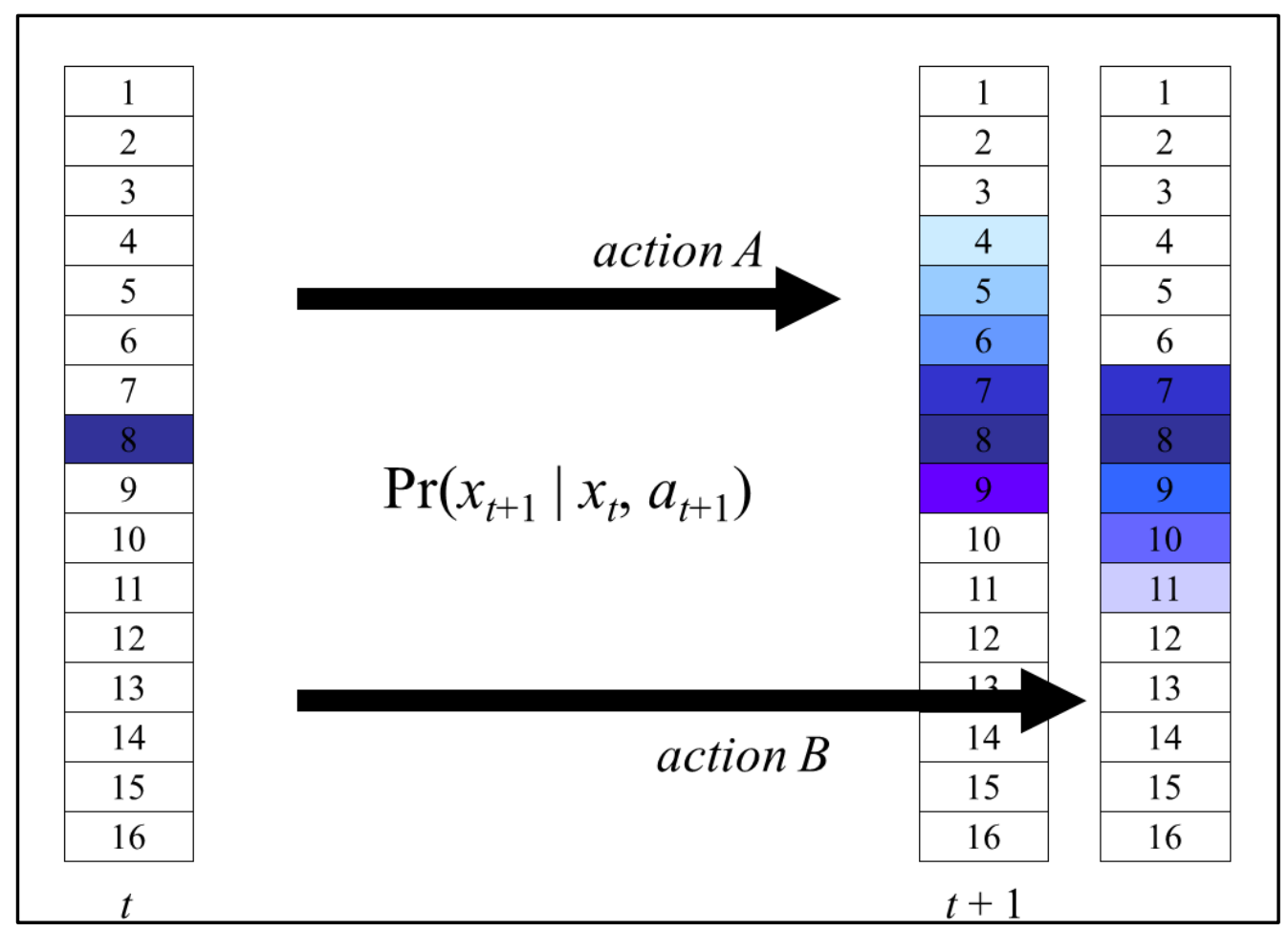

Figure 5. A pictorial simplification of the state transition probability model. On the left, the 16 vegetation states that a management unit can be in at any one time are represented by a vector at time $t$. On the right, the 16 vegetation states that a management unit can be in at time $t+1$ also are represented by vectors. The state transition probability model predicts the probability of being in each of the 16 different vegetation states at time $t+1$ given the starting vegetation state at time $t$ and the management action implemented between time $t$ and $t+1$ (we index this as time $t+1$ ); that is, $\operatorname{Pr}\left(x_{t+1} \mid x_{t}, a_{t+1}\right)$. In this example, assume the management unit is in vegetation state 8 at time $t$ (indicated by the shading). If we assume a management action, action $a_{t+1}=\mathrm{A}$, is taken just prior to time $t+1$, then we get a prediction of the probability of being in each of the 16 vegetation states at time $t+1$. We have used shading to indicate probability, where darker shading represents higher probability and lighter shading represents lower probability. Alternatively, if we assume that management action $a_{t+1}=B$ is carried out, then we make a different prediction about how the vegetation state of the unit will respond to that management action, and we have a different prediction of the probability of the resulting vegetation state at time $t+1$. 


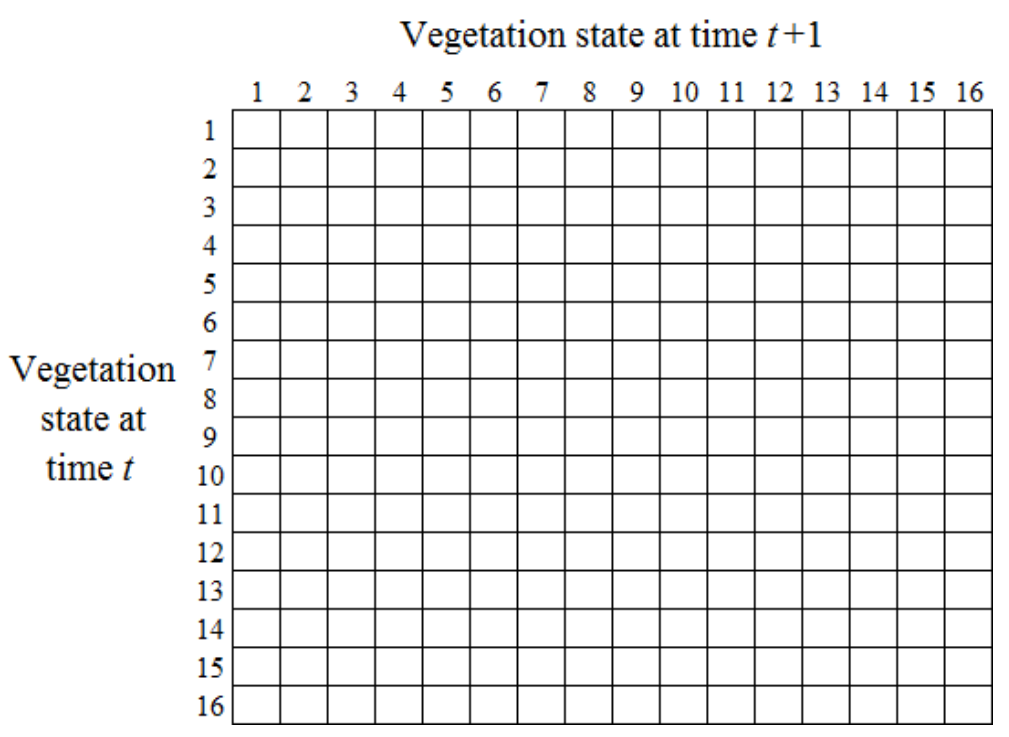

Figure 6. A single $16 \times 16$ matrix of the state transition probability model. The matrix contains all possible transitions from each of the 16 vegetation states at time $t$ to each of the 16 vegetation states at time $t+1$. The matrix, with each of the 256 cells containing a probability, describes the probability of transitioning from vegetation state $x$ at time $t$ to vegetation state $x$ at time $t+1$, given a particular management action that is applied. 


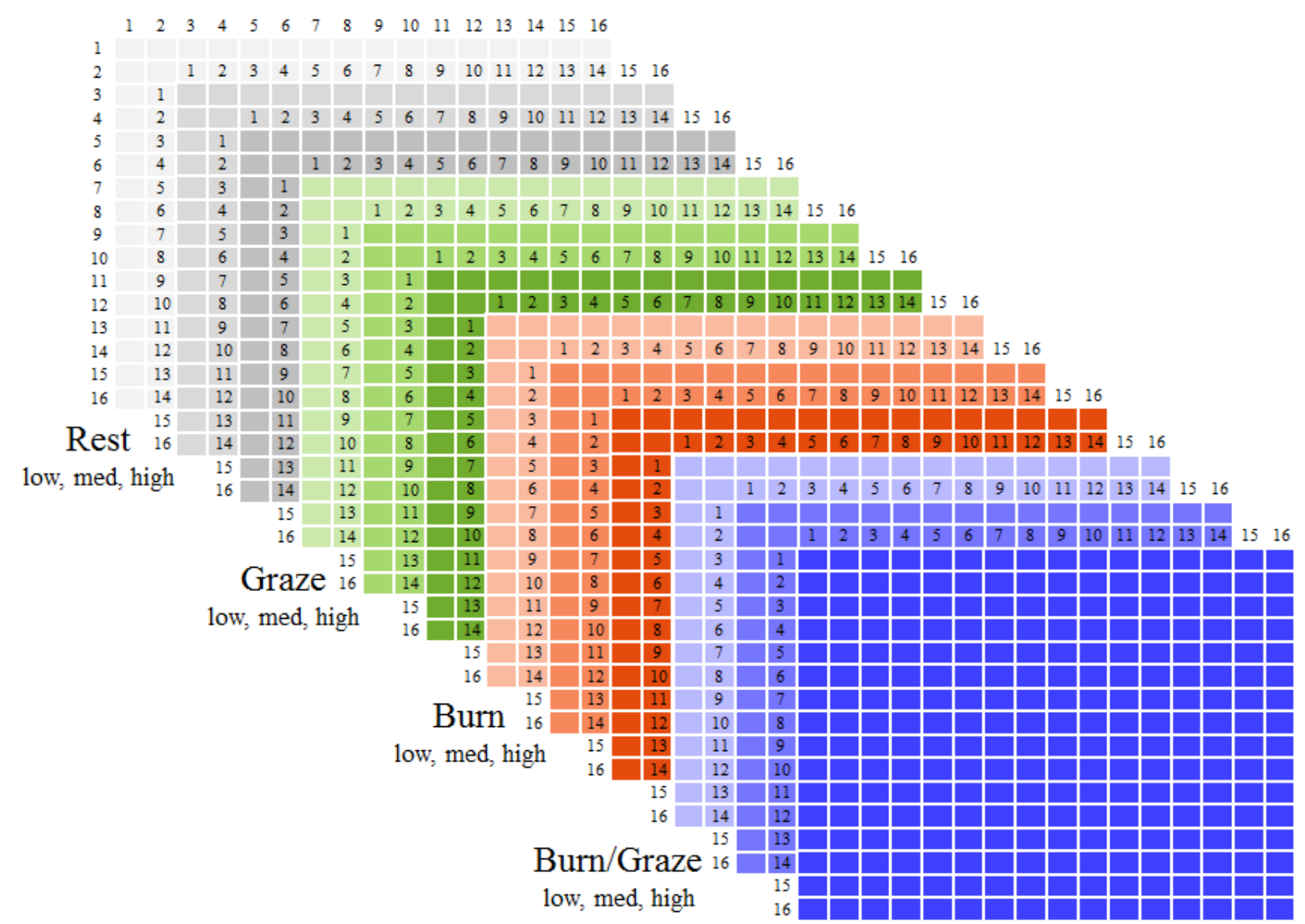

Figure 7. A simplistic representation of a complete mixed-grass model, consisting of twelve, $16 \times 16$ matrices: one for each of the four alternative management actions (rest, graze, burn, burn/graze) at each of the three defoliation levels (low, medium, high). The tallgrass model has the same structure, but the matrices are for the tallgrass alternative management actions (rest, graze within window, burn within window, defoliate).

\subsubsection{Model Parameterization}

We parameterized the models based on expert opinion. While using empirical knowledge to parameterize the models would be preferable to expert judgment, in the absence of data, models based on expert opinion provide a reasonable starting point and serve as a useful placeholder until informative data can be accumulated. For each grass type, we used expert elicitation to parameterize the 12 matrices of a foundational baseline model. The baseline model embodied a specific hypothesis of vegetation response to management actions. By manipulating parameters within the baseline model, we developed other models to represent alternative, competing hypotheses of response (see section 4.6 Representing Structural Uncertainty through Alternative Models). Details of the elicitation process for the baseline model, including the general approach and rationale for specific choices can be found in appendix $3 \mathrm{a}$ and digital appendix $7 \mathrm{a} 1$ for mixed-grass units and appendix $3 \mathrm{~b}$ and digital appendix $7 \mathrm{~b} 1$ for tallgrass units. We briefly describe the general approach here.

To more easily elicit information, we made the simplifying assumption that probability of transition among dominance classes was independent of level of native prairie cover. This assumption allowed us to break the elicitation exercise into two sections that were individually easier for our respondents to approach than an exercise designed around a fully dependent structure. In the first 
section, we elicited predictions about the change in percent native cover given scenarios of each of the four dominant invader cases for each of the four alternative management actions. We elicited this information with four different scenarios that capture the low and high ends of percent native cover and defoliation level history (table 10A). In the second section, we used the values elicited in the first section to derive predictions about the change in dominance of invasive species in scenarios of each of the four dominant invader cases for each of the four alternative management actions. We derived this information with two scenarios that capture the low and high end of defoliation level (table 10B).

Table 10. Skeleton of the format used to elicit the information necessary to parameterize the mixed-grass vegetation model.

[The format consisted of two sections. A, The first section was used to elicit predictions about the change in percent native cover under 64 different scenarios: 2 percent-native-cover starting states ( 80 percent and 20 percent), 2 defoliation level starting states (low and high), 4 dominant invader states ( $\mathrm{SB}=$ smooth brome, $\mathrm{CO}=$ co-dominant smooth brome and Kentucky bluegrass, $\mathrm{KB}=$ Kentucky bluegrass, $\mathrm{RM}=$ remainder), and 4 alternative management actions $(\mathrm{R}=$ rest, $\mathrm{G}=$ graze, $\mathrm{B}=$ burn, $\mathrm{BG}=$ burn/graze). $B$, The second section was used for predictions about the change in dominance of invasive species under 32 different scenarios: 2 defoliation level starting states (low and high), 4 dominant invader states (SB, CO, KB, RM), and 4 alternative management actions (rest, graze, burn, burn/graze). $\mathrm{NP}=$ native prairie]

$A$, First section used for eliciting change in percent native cover

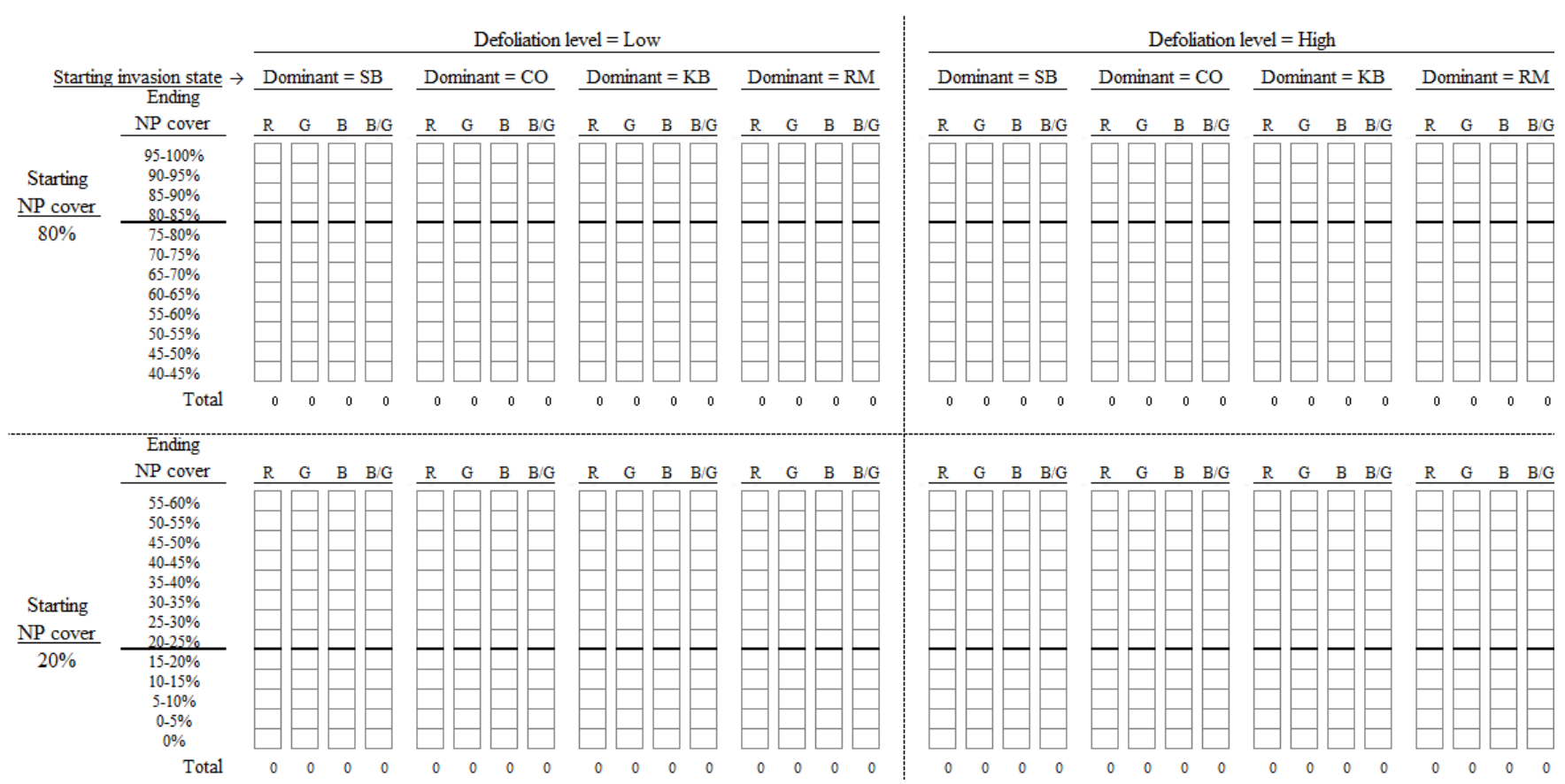

$B$, Second section used for predicting change in dominant invader state
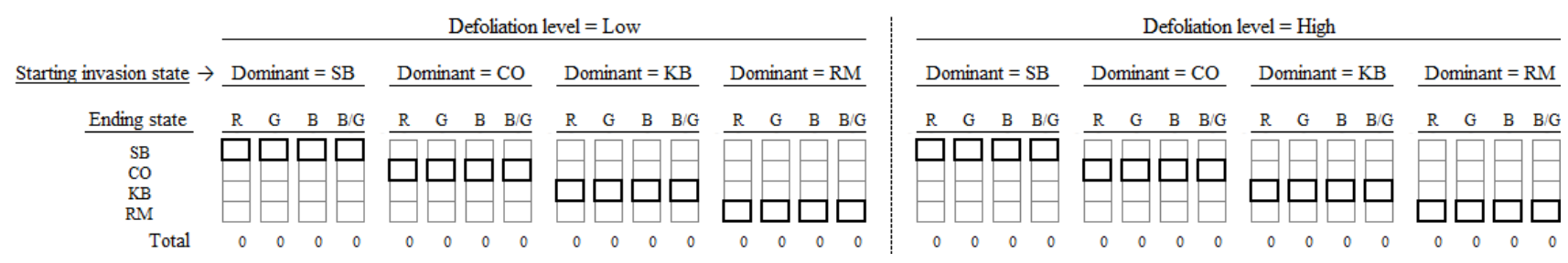
Despite the simplifications used (the independence assumption and the use of low/high state levels) the elicitation format was involved and took a considerable investment of time to complete; for this reason, one expert was selected to complete the elicitation for the mixed-grass model and one expert was selected to complete the elicitation for the tallgrass model. These experts were nominated from within the FWS part of the Science Team. For the change in native cover (table 10A), the expert was asked to distribute 100 points (in five-point increments) to the bins based on his prediction of the probability of the percent native cover in the next time step for each of the 64 different scenarios (that is, given the specific combination of starting percent native cover, starting defoliation level, starting dominance state, and management action applied). This process yielded a distribution of 20, five-point allotments among the ending native cover class bins for each of the 64 scenarios. Details of the approach are in appendix $3 \mathrm{a}$ for mixed-grass and appendix $3 \mathrm{~b}$ for tallgrass. Change in dominance (table $10 \mathrm{~B}$ ) proved difficult for the experts to provide useful predictions; rather, the 100 points were distributed to the bins for each of the 32 different scenarios (that is, given the specific combination of starting defoliation level, starting dominance state, and management action applied) based on rules and calculations we developed that directly link to the values elicited in the first section of the exercise (table 10A). As before, this process yielded a distribution of 20, five-point allotments among the four ending dominant invader classes for each of the 32 scenarios. The method, rules, and calculations are detailed in appendix $3 \mathrm{a}$ for mixed-grass and appendix $3 \mathrm{~b}$ for tallgrass, whereas the detailed calculations can be viewed in digital appendix $7 \mathrm{a} 1$ for mixed-grass and digital appendix $7 \mathrm{~b} 1$ for tallgrass. The completed elicitations for the mixed-grass baseline model and the tallgrass baseline model are in appendix 4a1 and appendix 4b1, respectively.

We fit linear models to the values that we elicited, and we simulated these models to derive the transition probabilities for the baseline state transition probability model (SAS code; digital appendix 8). The elicited native cover responses (20 predictions of native cover outcome per scenario) were converted to empirical logits, and they were regressed on a saturated linear model containing initial native cover state (continuous predictor), defoliation index (continuous), dominant invader (categorical predictor; four levels), management action (categorical; four levels), and all possible interactions among the predictors. For each combination of native cover system states (four; 0-30 percent, 30-45 percent, 45-60 percent, 60-100 percent), dominant invader states (four), defoliation levels (three), and management actions (four), we drew 100,000 random values of native cover percentage and defoliation index. For each simulated set of predictors, we generated a random prediction from the linear model, drawing the prediction (on the logit scale) from a normal distribution using the model's estimated standard error of the prediction. The simulated predicted value was then classified into the appropriate native cover state, and it served as one realization of transition from state $i$ at time $t$ to state $j$ at time $t+1$ under the given dominant invader state, defoliation level, and management action. Averaging across simulations provided estimated probabilities of transition between native cover states.

We used a similar approach for the dominant invader response. However, we treated the four dominant invader classes as a multinomial response in a linear logit model (Agresti, 2002), again using 20 predictions of dominant invader outcome per scenario. The model included defoliation index (continuous predictor), dominant invader (categorical predictor; four levels), and management action (categorical; four levels) as predictor variables, as well as all possible interactions among the terms (that is, a saturated model). For each combination of dominant invader states (four), defoliation levels (three), and management actions (four), we drew 100,000 random values of defoliation index. For each simulated set of predictors, we generated a prediction of class outcome probability (that is, probability of membership in each dominant invader class) from the logit model. To approximate prediction error in this vector of probabilities $\left(\hat{p_{i}}\right)$, we generated beta-distributed random variates $\left(\alpha=20^{*} \hat{p_{i}}, \beta=20^{*}(1-\right.$ 
$\left.\hat{p_{i}}\right)$ ) for each probability component and we normalized the outcomes to result in a probability distribution for the four components. This approach approximated draws made from a four-component Dirichlet distribution. The resulting vector thus served as one realization of transition from dominant invader class $i$ at time $t$ to each of the four classes at time $t+1$ under the given defoliation level and management action. Averaging across simulations provided estimated probabilities of transition between dominant invader states.

Because we made the simplifying assumption that probability of transition among dominance classes was independent of level of native prairie cover, and vice versa, we constituted the full vegetation dynamics model by cross-multiplying estimated transition probabilities for native cover state with estimated transition probabilities for dominant invader states. That is, for each native cover state (0-30 percent, 30-45 percent, 45-60 percent, 60-100 percent), dominant invader state, defoliation level, and management action, we multiplied each of the four native cover transition probabilities by each of the four dominant invader class transition probabilities. This operation yielded a vector of 16 transition probabilities, comprising a single row of the baseline matrix model (fig. 5).

The resulting populated transition matrices for the mixed-grass baseline model and tallgrass baseline model are located in digital appendix $6 \mathrm{a}$ and digital appendix $6 \mathrm{~b}$, respectively.

\subsubsection{Model Prediction}

A complete vegetation model predicts the consequences of the management action taken, with regard to the vegetation state, given the initial vegetation state and defoliation level. The model requires as input the vegetation state and defoliation level of the unit at time $t$, as well as the management action implemented on the unit at time $t+1$. The prediction the model provides is a probability distribution of outcome among the 16 vegetation states of the unit at time $t+1$, given the input.

$\operatorname{Pr}\left(x_{t+1} \mid x_{t}, l_{t}, a_{t+1}\right)$

where

$$
\begin{aligned}
& x=\text { vegetation state } \\
& l=\text { defoliation level } \\
& a=\text { management action } \\
& t=\text { time }
\end{aligned}
$$

\subsubsection{Defoliation Model}

Similar to the vegetation model, we use a state transition probability model to describe how a unit transitions between defoliation states in a single time step given the management action applied. The defoliation transition model consists of two $7 \times 7$ matrices, one for if the unit is rested and one for if the unit is defoliated (that is, treated by at least one nonrest action) during the time step (table 11). We populated the two transition matrices by calculating the probabilities of transitioning from each of the seven defoliation states at time $t$ to each of the seven defoliation states at time $t+1$ (that is, a one-year time step), given the unit received a rest (no action) and given the unit received at least one defoliation action during that time step (table 11). To calculate these probabilities, we followed 6 steps: (1) determined the 128 possible seven-year past management combinations of rest and defoliation actions at time $t$; (2) assumed 2 scenarios - the unit was rested or the unit was defoliated in the next time step $t+1$; (3) determined the seven-year past management combination for each of the 128 possible combinations at time $t+1$ under the 2 scenarios; (4) calculated the years since last defoliation and defoliation index for all 128 combinations at time $t$ and time $t+1$ under both scenarios; (5) classified years since last defoliation into 1 of the 3 categories $(5+, 2-4,1)$, classified the defoliation index into 1 of the 3 levels 
(low, med, high), and combined these to determine the defoliation state (1-7) for all 128 combinations at time $t$ and time $t+1$ under both scenarios; and (6) calculated the frequency of transitions between the defoliation states from time $t$ to time $t+1$ under both scenarios. While we keep track of each unit's sevenyear management history to accurately record its current defoliation state, the transition probabilities thus produced allowed probabilistic predictions to be made about a unit's future defoliation state without having to know its complete defoliation history. For the later step of dynamic optimization of the management models, the ability to disregard the detailed defoliation history was crucial, as the optimization methods are quickly overwhelmed by too much state detail.

Table 11. Probabilities of transitioning among the seven defoliation states in a one-year time step, given the choice of either $A$, rest or $B$, defoliation (that is, action other than rest) as the management action within that time step. [For ease of reading, defoliation states are indicated by the combination of the classified years since last defoliation $(5+, 2-4,1)$ and defoliation level (low, med, high), rather than simply numbered 1-7]

\section{$A$, Rest}

Defoliation state at time $t+1$

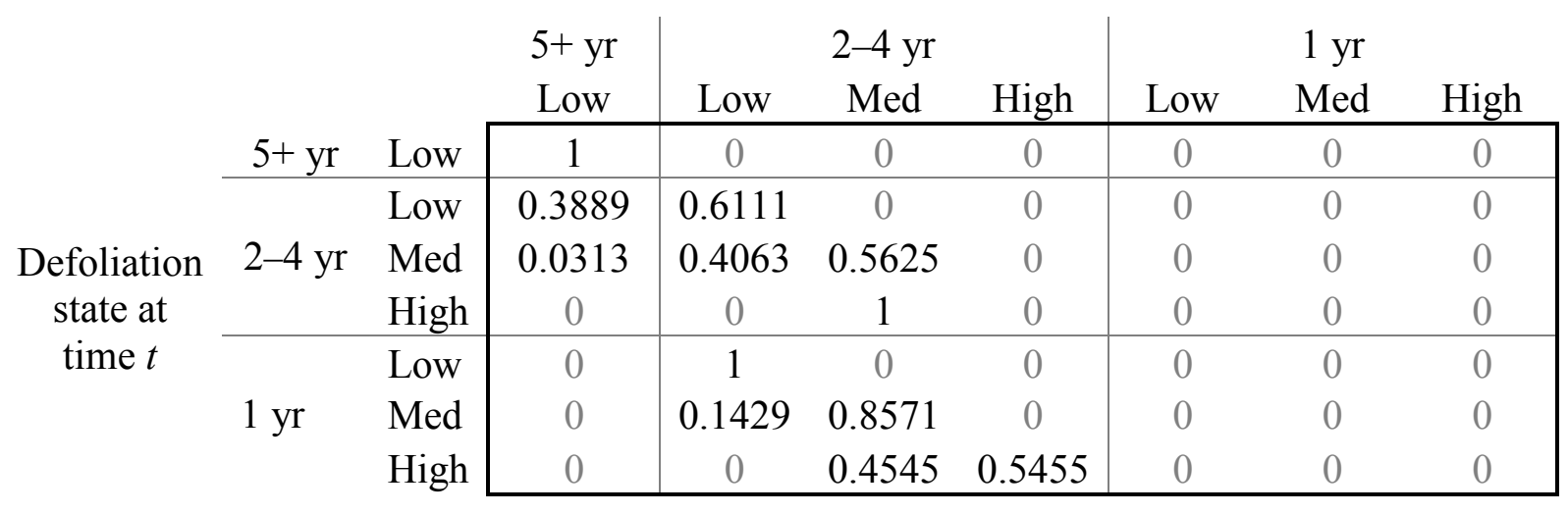

$B$, Defoliation

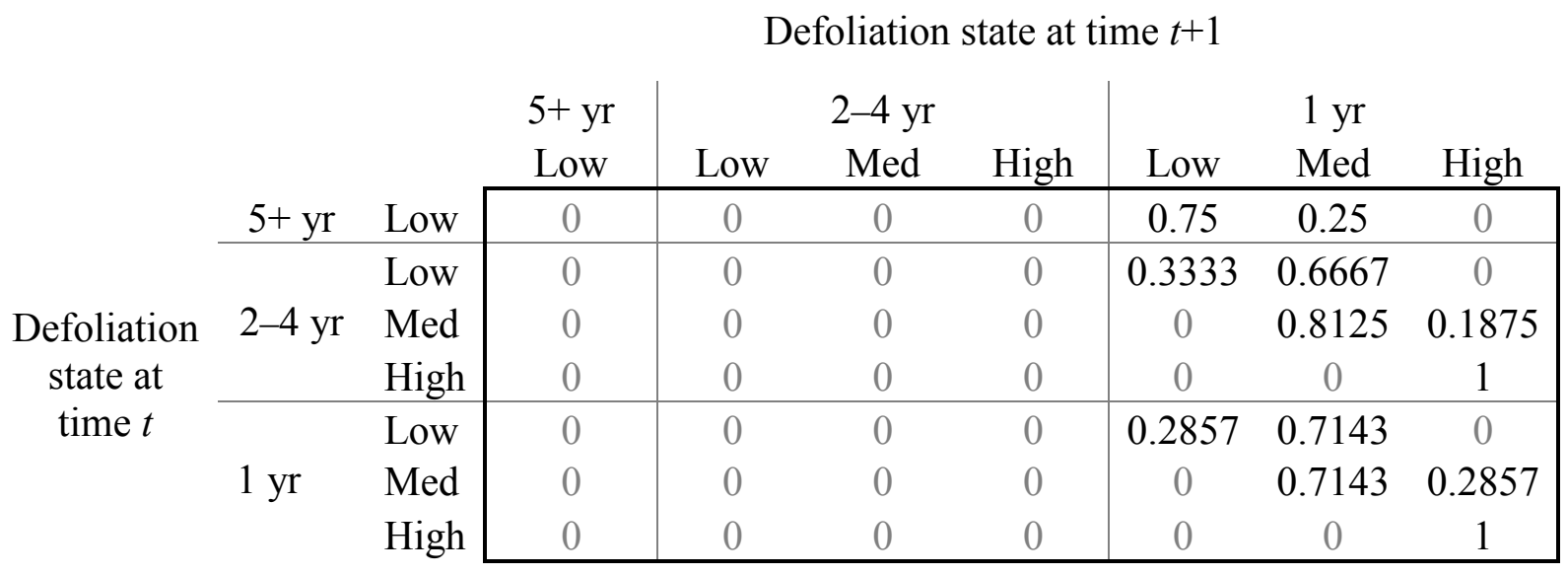




\subsubsection{Synthesis of the Vegetation and Defoliation Models}

The vegetation matrices and the defoliation matrices work together in a two-step process to predict the vegetation state and the defoliation level in the next time step. The predictions are used in optimization analysis, in which management actions are compared based on their consequences in the future; and they are used in model weight updating, in which predictions by different models are evaluated against one another based on observed outcomes. The vegetation transition probability matrices take as input the current vegetation state, defoliation level, and management action. Given these inputs, they provide as output the probability of being in the various vegetation states in the next time step (see notation below, vegetation model). The defoliation transition probability matrices take as input the current classified years since the unit was last defoliated, defoliation level, and management action. Given these inputs, they provide as output the probability of being in the various defoliation states in the next time step (see notation below, defoliation model).

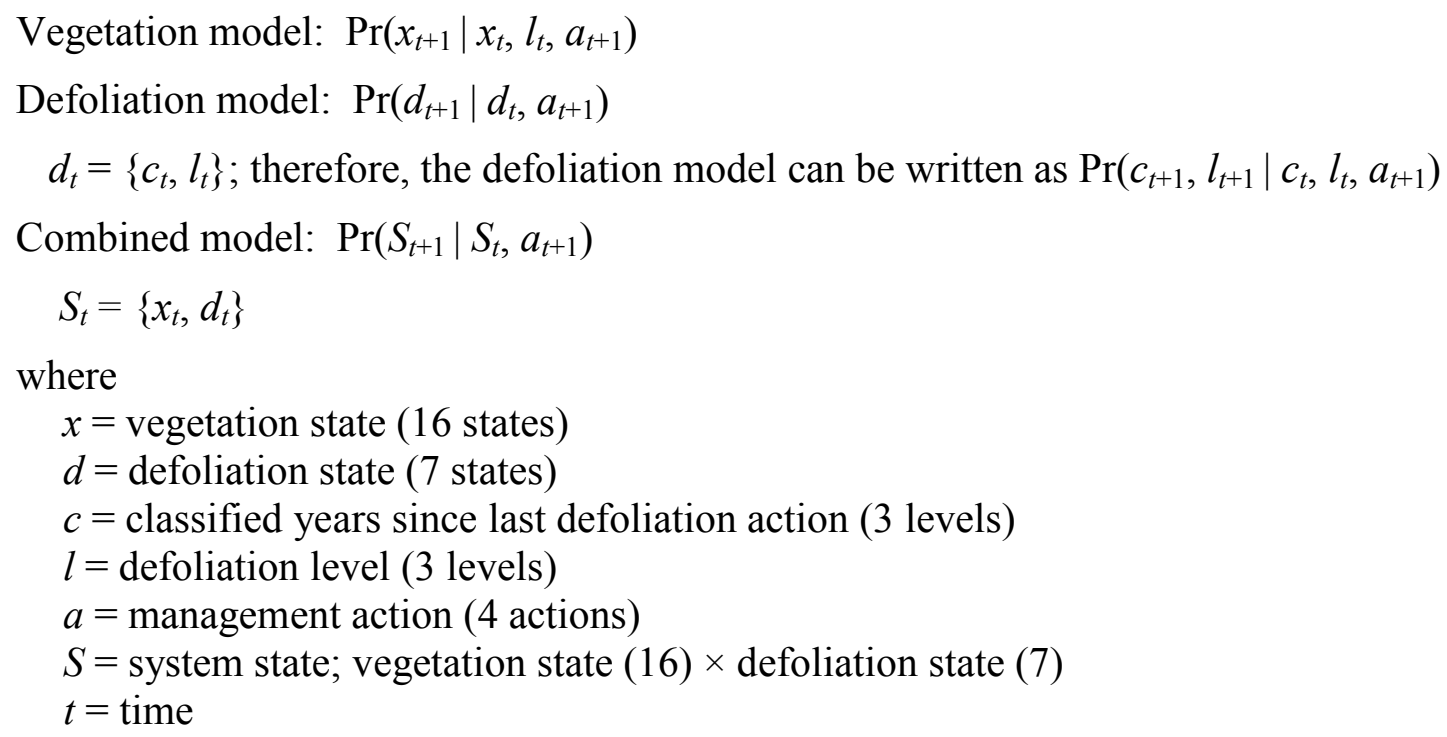

\subsection{Representing Structural Uncertainty Through Alternative Models}

\subsubsection{Structural Uncertainty}

There is uncertainty about the behavior of the system; that is, we are uncertain how the vegetation components of interest will respond to implemented management actions. This uncertainty makes decision making difficult because which management action is best to apply to a unit depends on how we expect the system to behave. We began to identify uncertainties about system behavior through discussion and elicitation with the group of cooperators at the NPAM kick-off meeting of July 2008. To initiate the group discussion, we asked the cooperators the following question: What makes it difficult to decide each year which management action to implement? We discussed types of information, when lacking, that hindered decision making. We also explored areas of disagreement about system behavior among cooperators. We compiled a list of uncertainties and through extensive follow-up discussion with the Science Team, we pinpointed the key uncertainties the team believed to be the major impediments to decision making and the biggest point of disagreement among cooperators. Some of these key uncertainties are specific to grass type and thus are outlined below as applying to both mixedgrass and tallgrass or applying to only tallgrass. 


\subsubsection{Mixed-Grass and Tallgrass}

Three key uncertainties revolved around whether the effect of management actions depends on the following:

(1) The type of dominant invader- Does the effectiveness of management, and thus best choice of management action, depend on whether the dominant invader is smooth brome, Kentucky bluegrass, co-dominant smooth brome and Kentucky bluegrass, or something else?

(2) The past defoliation history_-Does the effectiveness of management, and thus best choice of management action, depend on whether the unit has been aggressively managed or extensively rested in the recent past?

(3) The level of invasion-Does the effectiveness of management, and thus best choice of management action, depend on how invaded the unit is? Is there a threshold of invasion, above which active management does not effectively combat invasive species in favor of cover by native species, such that the unit has succumbed to invasive species and there is little hope of reestablishing native cover through application of the available alternative management actions?

\subsubsection{Tallgrass Specific}

In addition to the three uncertainties outlined above, cooperators identified two more key uncertainties that are specific to managing tallgrass prairie units. Burning during the spring when smooth brome is at a critical growth stage and native warm-season grasses are not yet active is believed effective for reducing smooth brome and invigorating native warm-season grasses (Willson and Stubbendieck, 2000). For purposes of NPAM, we refer to the critical time described by Willson and Stubbendieck (2000) as the cool-season window, and we assume said window exists not only in the spring, but also in the fall (see section 4.3.2.2 Tallgrass). The following uncertainties were of high interest to tallgrass cooperators because their resolution has implications for how cooperators might be able to take advantage of management opportunities, other than burning during the cool-season window, to combat smooth brome.

(4) Can grazing within the cool-season window be used as an effective surrogate for burning within the window when smooth brome is the dominant invader?

(5) If management cannot be carried out within the window, is it better to apply the management outside of the window or to rest and wait until the next window of opportunity to implement active management?

\subsubsection{Alternative Models}

The goal of managing under an adaptive management framework is to reduce uncertainty about response of the system to management actions, thereby improving understanding of how the system works and providing a basis for managers to make better management decisions. To do this, we must explicitly frame the uncertainties that we want to learn about through alternative models that represent our different notions of how we think the system responds to management. Alternative models make different predictions about the consequences of applying a given management action in terms of the resulting vegetation state, which is the measureable attribute we use to represent our system. The predictions are based on our different hypotheses of how we think the system responds to management, whereas the hypotheses revolve around the key areas of uncertainty outlined above that were identified as hindering managers' ability to make management decisions. 


\subsubsection{Mixed-Grass}

We developed four alternative models to capture the three key uncertainties about the behavior of the mixed-grass system. These four models propose different hypotheses of system response to applied management actions with respect to the three uncertainties: (1) type of dominant invasive, (2) past management defoliation level, and (3) level of invasion. The models build on each other such that comparison of sequential models isolates each of the three identified uncertainties (fig. 8).

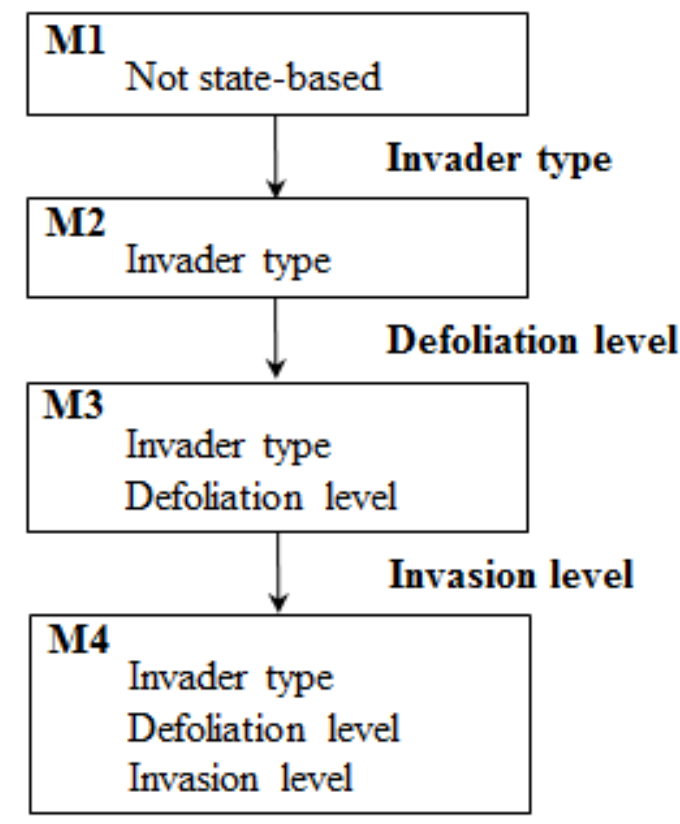

Figure 8. Diagram of the four alternative mixed-grass models. The models build on each other such that comparison between sequential models isolates each of the three identified uncertainties depicted here in bold.

Model 1 predicts that all active management is equally effective and better than rest regardless of system state; that is, regardless of the vegetation state (native cover level and dominant invader) and regardless of the defoliation state, this model predicts that all active management actions (that is, graze, burn, and burn/graze) are equally effective at combatting invasive cover in favor of native cover and are more effective than doing nothing (that is, rest). This model leads to a management strategy that carries out defoliation (in any form) on a fixed recurring cycle without respect to current vegetation status. Thus, although the biology expressed in this model is independent of vegetation and defoliation state, we make use of the 'years since last defoliation' part of the defoliation state variable as the temporal trigger for management action.

Model 2 is based on the supposition that the response of the system (that is, the vegetation state of the unit) to management actions depends on the type of invader that is dominant. The conceptual model postulates that management actions are differentially effective depending on the dominant invader, as follows:

- When SB is the dominant invader, we postulate that:

$\circ \quad($ Burn $/$ graze $=$ graze $)>$ burn $>$ rest

- When SB and KB are co-dominant (CO), we postulate that:

$\circ$ Burn $/$ graze $>($ burn $=$ graze $)>$ rest 
- When $\mathrm{KB}$ is the dominant invader, we postulate that:

○ $($ Burn $/$ graze $=$ burn $)>$ graze $>$ rest

- When some other type is the dominant invader (RM), we postulate that:

$\circ \quad($ Burn $/$ graze $=$ burn $=$ graze $)>$ rest

where ' $=$ ' indicates that either action is equally effective and ' $>$ ' indicates that the action(s) to the left of the sign is more effective than the action(s) to the right.

Model 3 builds on Model 2 and predicts that in addition to the relevance of the dominant invader, past management history of the unit plays a role in the response of the system to current management actions. With regard to the dominant invader, Model 3 proposes the same relationship as outlined in Model 2. With regard to past management history, Model 3 posits that a history of frequent defoliation creates momentum, such that rest is less detrimental and active management is more effective in comparison with these actions carried out on units having histories of infrequent defoliation. The premise is that rest is less detrimental (in terms of loss of native cover to non-native species) if carried out on a unit that has a high defoliation level during the previous seven-year window than if carried out on a unit that has a low defoliation level. Also, active management actions are hypothesized to be more effective (in terms of combating non-native species in favor of native cover) if carried out on a unit that has a high defoliation level, than if carried out on a unit that has a low defoliation level; this last concept, however, is assumed to behave in a density dependent manner such that it is more expressive at low native cover (e.g., 20 percent) than high native cover (e.g., 80 percent).

Model 4 builds on Model 3 and proposes that system response to management depends not only on invader type and defoliation history, but also on the level of invasion. Model 4 predicts that management effectiveness declines as the level of invasion increases, and that at high levels of invasion (e.g., only 20 percent native cover), active management is no more effective than rest.

\subsubsection{Tallgrass}

We developed six alternative models to capture the five key uncertainties about the behavior of the tallgrass system. These six models propose different hypotheses of system response to applied management actions with respect to the five uncertainties: (1) type of dominant invasive, (2) past management defoliation level, (3) level of invasion, (4) effectiveness of grazing within the window compared with burning within the window when smooth brome is the dominant invader, and (5) effectiveness of applying a management action outside of the window (that is, defoliate) compared with rest. The models build on each other such that comparison of sequential models isolates each of the three identified uncertainties (fig. 9). 


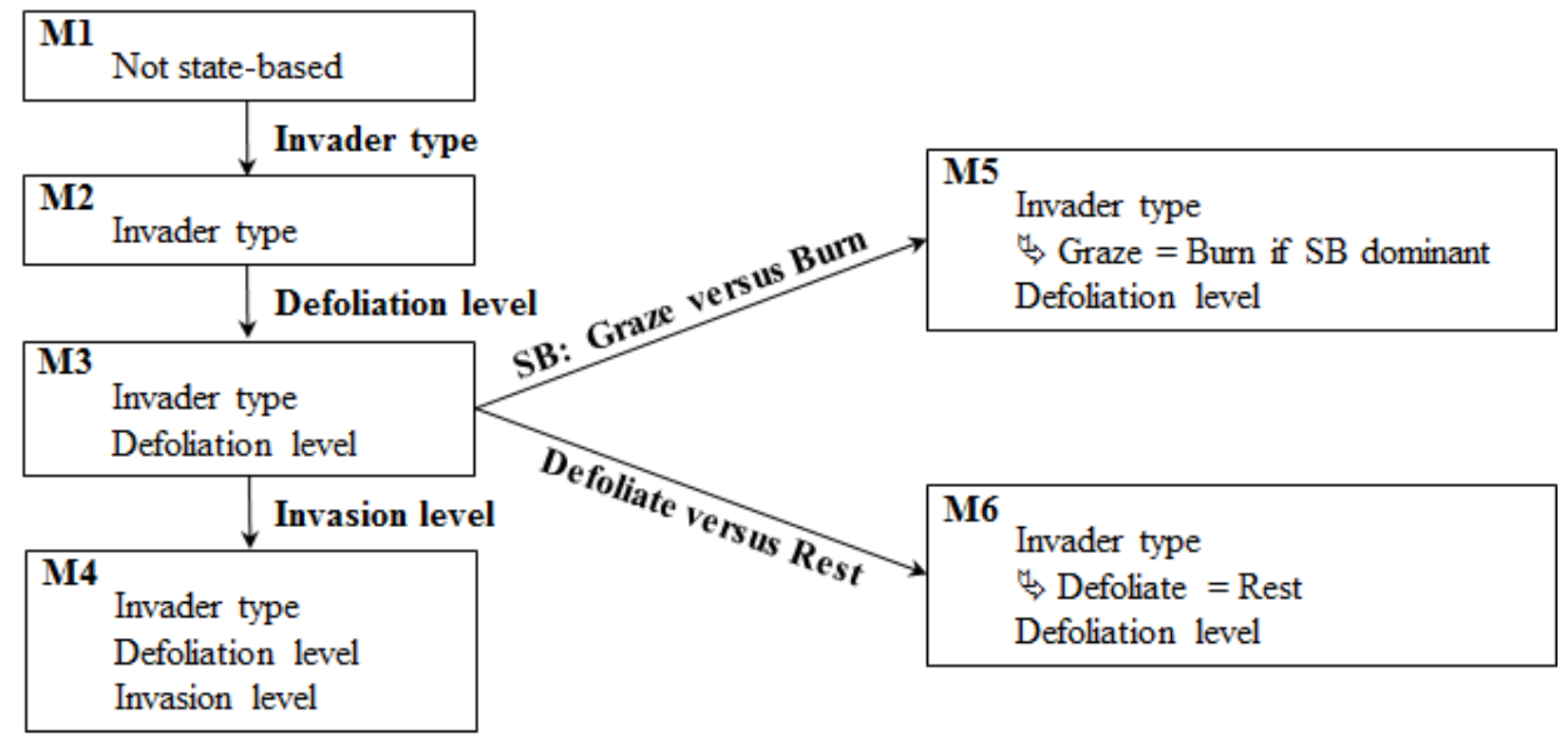

Figure 9. Diagram of the six alternative tallgrass models. The models build on each other such that comparison between paired models (M1|M2, M2|M3, M3|M4, M3|M5, M3|M6) isolates each of the five identified uncertainties depicted here in bold. Note that Model 5 and Model 6 branch off from, and are variants of, Model 3. $\mathrm{SB}=$ smooth brome. Graze=graze within window. Burn=burn within window.

Model 1 predicts that all active management is equally effective and better than rest regardless of system state; that is regardless of the vegetation state (native cover level and dominant invader) and regardless of the defoliation state, this model predicts that all active management actions (that is, graze within window, burn within window, and defoliate) are equally effective at combating invasive cover in favor of native cover and are more effective than doing nothing (that is, rest). This model leads to a management strategy that carries out defoliation (in any form) on a fixed recurring cycle without respect to current vegetation status. Thus, while the biology expressed in this model is independent of vegetation and defoliation state, we make use of the 'years since last defoliation' part of the defoliation state variable as the temporal trigger for management action.

Model 2 predicts that the response of the system (that is, the vegetation state of the unit) to management actions depends on the type of invader that is dominant. The conceptual model proposes that with the exception of dominance by invaders other than smooth brome and Kentucky bluegrass (that is, RM dominant invader), the relative order of management effectiveness is burn within window followed by graze within window, followed by defoliate, followed by rest (see below).

- When SB, SB and KB (CO), or KB are dominant, we postulate that:

○ Burn within window $>$ graze within window $>$ defoliate $>$ rest

- When RM is dominant, we postulate that:

$\circ \quad($ Burn within window $=$ graze within window $=$ defoliate $)>$ rest

Although the order of the management action effectiveness is proposed to be the same, the level of effectiveness of these management actions is predicted to vary with the type of dominant invader. In general, grazing within the window is hypothesized to be more effective against smooth brome than against Kentucky bluegrass. 
Model 3 builds on Model 2 and predicts that in addition to the relevance of the dominant invader, past management history of the unit plays a role in the response of the system to current management actions. With regard to the dominant invader, Model 3 proposes the same relationship as outlined in Model 2. With regard to past management history, Model 3 posits that a history of frequent defoliation creates momentum, such that rest is less detrimental and active management is more effective. The premise is that rest is less detrimental (in terms of loss of native cover to non-native species) if carried out on a unit that has a high defoliation level during the previous seven-year window than if carried out on a unit that has a low defoliation level. Also, active management actions are hypothesized to be more effective (in terms of combating non-native species in favor of native cover) if carried out on a unit that has a high defoliation level, than if carried out on a unit that has a low defoliation level; this last concept, however, is assumed to behave in a density dependent manner such that it is most expressive at low native cover (e.g., 20 percent) than high native cover (e.g., 80 percent).

Model 4 builds on Model 3 and proposes that system response to management depends not only on invader type and defoliation history, but also on the level of invasion. Model 4 predicts that management effectiveness declines as the level of invasion increases, and that at high levels of invasion (e.g., only 20 percent native cover), active management is no more effective than rest.

Model 5 is a variant of Model 3. Like Model 3, Model 5 is based on the supposition that the response of the system to management depends on the type of dominant invader and the past defoliation history of the unit. Model 5, however, proposes a different idea about the differential effectiveness of the management actions with dominant invader. Model 5 introduces the idea that under conditions of smooth brome dominance, grazing within the window is just as effective as burning within the window; thus, when smooth brome is dominant, grazing within the window is proposed to be more effective under Model 5 than it is under Model 3. Comparing Model 5 to Model 3 isolates the fourth uncertainty, which concerned whether grazing within the window could be used as a surrogate for burning within the window when smooth brome is dominant. This question is of practical importance to tallgrass cooperators who may not have access to prescribed fire on some management units or who are unable to carry out a burn during the phenological window (e.g., due to unfavorable weather conditions).

Model 6 also is a variant of Model 3 and also is based on the premise that the response of the system to management depends on the type of dominant invader and the past defoliation history of the unit. Unlike Model 3, however, Model 6 proposes a different relative order of management effectiveness. Specifically, where Model 3 posits that applying an active form of management outside of the window (that is, defoliate) is more effective than rest, Model 6 postulates that implementing management outside of the window is equivalent to rest (see below).

- When SB, SB and $\mathrm{KB}(\mathrm{CO})$, or $\mathrm{KB}$ are dominant, we postulate that:

$\circ$ Burn within window $>$ graze within window $>$ defoliate $=$ rest

- When RM is dominant, we postulate that:

$\circ \quad($ Burn within window $=$ graze within window $)>($ defoliate $=$ rest $)$

Comparing Model 6 to Model 3 puts focus on the fifth uncertainty about the effectiveness of management outside of the window. The assumption for all models is that management within the window is superior to management outside of the window; however, the question remains whether management outside of the window is superior to rest. This question is addressed by the comparison of Model 6 with Model 3. This question is of practical importance to tallgrass cooperators, who may have difficulty in meeting the temporal requirements for management within the phenological windows but who nevertheless desire for their efforts outside of these windows to have conservation significance. 


\subsubsection{Parameterization of Alternative Models}

We elicited values from FWS members of the Science Team to parameterize a baseline vegetation model for mixed-grass and tallgrass (see section 4.5.1.2 Model Parameterization); we used Model 3 as the baseline model for both grass types for purposes of the elicitation exercise. The predictions of the baseline models (mixed-grass Model 3 and tallgrass Model 3) are based on the conceptual models of each grass type described in the previous section (section 4.6.2 Alternative Models); these conceptual models reflect one of the alternative hypotheses that the Science Team proposed with regard to how they think the system responds to management actions. Eliciting the values for the baseline models of each grass type provided us with the necessary information on all of the relationships within the elicitation sheet including extremes of starting native cover (80 percent compared to 20 percent), low compared to high defoliation level, four different dominant invaders, and four alternative management actions. Given the elicited values for all of the relationships in the baseline model, and given the conceptual models for the baseline and the alternative models, it was a straightforward task to alter the elicitation values of the baseline model to be consistent with and faithfully represent the relationships expressed within each alternative model. We describe how we altered the values per alternative model in appendix $5 \mathrm{a}$ for mixed-grass and in appendix $5 \mathrm{~b}$ for tallgrass. The dominance section of the elicitation sheet (refer to table 10B) for each alternative model was completed using the same methods as used for the baseline model; that is, we used the modified elicited values for the section of the sheet regarding change in native cover to calculate the dominance section according the rules and calculations previously set forth (refer to appendixes $3 \mathrm{a}$ and $3 \mathrm{~b}$ ). The spreadsheet with the calculations themselves can be viewed in digital appendixes $7 \mathrm{a}(7 \mathrm{a} 2,7 \mathrm{a} 3,7 \mathrm{a} 4)$ for mixed-grass and digital appendixes $7 b(7 b 2,7 b 3,7 b 4,7 b 5,7 b 6)$ for tallgrass. The completed elicitation sheets for the mixed-grass alternative model set and the tallgrass alternative model set are in appendixes 4a1-4a4 and appendixes 4b1-4b6, respectively.

Just as for the baseline models, we used the raw values in the elicitation sheets as input for multinomial logit models, and we simulated these models to derive the transition probabilities for the state transition probability matrices for each of the alternative models. The resulting populated transition matrices for the mixed-grass alternative model set and tallgrass alternative model set are located in digital appendix $6 \mathrm{a}$ and digital appendix $6 \mathrm{~b}$, respectively.

\subsubsection{Model Predictions_-Implications of Alternative Models}

As described earlier, a vegetation model predicts the consequences of the management action taken, with regard to the vegetation state, given the initial vegetation state and defoliation level. The model requires as input the vegetation state and defoliation level of the unit at time $t$, as well as the management action implemented on the unit at time $t+1$. The prediction the model provides is a probability distribution of outcome among the 16 vegetation states of the unit at time $t+1$, given the input. Because alternative models are based on alternative hypotheses concerning how the system responds to management, it follows that alternative models make different predictions. Given the same input values for vegetation state and defoliation level at time $t$, and the same management action at time

$t+1$, the alternative models make different predictions about the outcome of management in terms of the resulting vegetation state. These different predictions stem from the competing hypotheses each model represents concerning how cooperators think the system responds to management. As such, the different predictions of the alternative models represent the range of structural uncertainty. 


\subsection{Partial Controllability}

A management action that is carried out is not always the targeted or intended management action. Circumstances such as unfavorable weather conditions, lack of resources, unpredictable access to burn crew or grazing contractors, to name a few, can affect the choice of management action and make it impossible to fully control what management action is implemented. This is a form of irreducible uncertainty that is known as partial controllability. It is not an uncertainty that can be reduced through the adaptive management framework; however, because it can affect the choice of optimal management action, we must explicitly recognize it in the decision framework. To do so, we needed to estimate the probability of each management action being implemented, given the management action that is recommended. We obtained these estimates by way of an elicitation exercise with the cooperators.

\subsubsection{Elicitation Exercise}

We sent an elicitation exercise to each of the 19 cooperating refuges/wetland management districts. The exercise consisted of an empty $4 \times 4$ matrix of the recommended management action (rows) by the implemented management action (columns; table 12). To elicit from cooperators the expected probabilities of executing each management action, given the management action that is recommended, they were asked to fill in the matrix with their best estimates. Because we expected that the frequency with which the recommended management action is carried out will vary by the management action itself, as well as by the particular unit, cooperators were asked to complete a single matrix separately for each of the management units they have enrolled in NPAM. We provided the following guidance for cooperators to observe when filling in the matrices per unit:

(1) Assume it has been a typical year and you have just received the recommended management action for each of your NPAM management units.

(2) Any given year, whether or not a particular management action can or will be carried out depends on many different factors; thus, take a long-term view of management and think in terms of average conditions when formulating probabilities for each unit.

(3) There is no right or wrong answer; we wish to capture your best estimate.

(4) Involve staff from your complex who are most familiar with the particular management units and with the management implementation on these units (e.g., biologists, managers, project leaders).

(5) Do not discuss the exercise with staff from other complexes, as we want responses to be independent among complexes.

(6) Probabilities per cell must be between 0 and 1 and must sum to 1 across the columns per row.

(7) If there is a management action that you cannot use on a particular unit, indicate this by putting a zero for the probability of that action being implemented when indicated as the recommended management action. 
Table 12. Blank $4 \times 4$ partial controllability matrix used by cooperators with mixed-grass units.

[The recommended management action is along the left (rows), whereas the implemented management action is across the top (columns). Per unit, cooperators provided their best estimate of the probability that each management action, if recommended, would be implemented, and if not implemented, the probability of each of the other three management actions being implemented in its place. Probabilities per cell must be between 0 and 1 , and probabilities across columns per row must sum to 1]

\section{Implemented action}

\begin{tabular}{|c|c|c|c|c|}
\hline \multirow[b]{2}{*}{$\begin{array}{l}\text { Recommended } \\
\text { action }\end{array}$} & \\
\hline & Rest & Graze & Burn & Burn/Graze \\
\hline \multicolumn{5}{|l|}{ Rest } \\
\hline \multicolumn{5}{|l|}{ Graze } \\
\hline \multicolumn{5}{|l|}{ Burn } \\
\hline Burn/Graze & & & & \\
\hline
\end{tabular}

We received cooperator responses for every NPAM management unit, which included 81 mixedgrass units and 38 tallgrass units (appendix 6). These responses indicated that three mixed-grass units were unsuitable for burn actions and six tallgrass units were unsuitable for graze actions.

\subsubsection{Method of Combining Responses}

For each grass type, we averaged the responses of cooperators to obtain conditional probabilities, $\operatorname{Pr}\left(a_{t+1}{ }^{\prime} \mid a_{t+1}\right)$, for use in optimization. To do this, we first excluded from analysis the data for three mixed-grass units where burning was not a feasible option and the data for six tallgrass units where grazing was not a feasible option. The data were organized in a nested structure in which management units were components of larger groupings (e.g., clusters of paddocks), which were themselves members of administrative units (e.g., refuges, WMDs), which themselves comprised NWR complexes. Responses within an organizational grouping tended to be more similar to each other than to responses outside of the grouping, partly reflecting effects that are dependent on scale (geographic and organizational) and partly reflecting the fact that a single cooperator typically provided data for all units in a complex. Furthermore, sample sizes were highly unbalanced among the various levels (e.g., 1-16 units per complex), suggesting that refuge complexes with many units would greatly influence an overall project-wide mean. For each recommended management action, we built a hierarchical Bayesian model to account for this structure and to minimize the effect of sample size imbalance on the overall mean. Each elicited unit-level probability for the $s^{\text {th }}$ component $\left(p_{i j k l}(s), s=\{1,2,3,4\}\right)$ was assumed to be drawn from a normal distribution with unobserved unit-specific mean $\alpha_{i j k l}^{(s)}$ and common variance $\tau_{1}^{(s)}$. The unit-level means, in turn, were assumed to arise from normal distributions with unobserved group-level means $\beta_{i j k}{ }^{(s)}$ and common variance $\tau_{2}{ }^{(s)}$. The hierarchy continued upward in this fashion, to the topmost level where complex-level means $\gamma_{i}^{(s)}$ were assumed to arise from normal distributions with overall grass type means $a^{(s)}$ and common variance $\tau_{5}^{(s)}$. 
Unit-level observation

$$
p_{i j k l}^{(s)} \sim \mathrm{N}\left(\alpha_{i j k l}^{(s)}, \tau_{1}^{(s)}\right)
$$

Unit-level mean

$\alpha_{i j k l}^{(s)} \sim \mathrm{N}\left(\beta_{i j k}^{(s)}, \tau_{2}^{(s)}\right)$

Group-level mean

$\beta_{i j k}^{(s)} \sim \mathrm{N}\left(\delta_{i j}^{(s)}, \tau_{3}^{(s)}\right)$

Org-level mean

$\delta_{i j}^{(s)} \sim \mathrm{N}\left(\gamma_{i}^{(s)}, \tau_{4}^{(s)}\right)$

Complex-level mean

$\gamma_{i}^{(s)} \sim \mathrm{N}\left(a^{(s)}, \tau_{5}^{(s)}\right)$

Region-level mean

$a^{(s)} \sim \mathrm{N}\left(\rho^{(s)}, \tau_{6}^{(s)}\right)$

Parent distribution

$\rho^{(s)} \sim \operatorname{uniform}(0,1)$

Variances

$\sqrt{\tau_{i}^{(s)}} \sim$ uniform $(0,1)$

We used Markov chain Monte Carlo sampling in WinBUGS (Lunn and others, 2000) to calculate posterior distributions for the $a^{(s)}$ parameters for each recommended management action. Thus, for a given recommended management action $a^{*}$, the means of the posterior distributions provided an estimate of $\operatorname{Pr}\left(a^{(1)} \mid a^{*}\right), \operatorname{Pr}\left(a^{(2)} \mid a^{*}\right), \operatorname{Pr}\left(a^{(3)} \mid a^{*}\right)$, and $\operatorname{Pr}\left(a^{(4)} \mid a^{*}\right)$, the probabilities of implementation of each of the management actions given $a^{*}$.

\subsubsection{Results}

After combining the elicited cooperator responses separately for the mixed-grass units and the tallgrass units, we obtained two matrices, one for each grass type (table 13).

Table 13. Partial controllability matrices for $A$, mixed-grass units and for $B$, tallgrass units.

[The values in the matrix for mixed-grass units includes 78 responses. The values in the matrix for the tallgrass units includes 32 responses. The responses were combined per grass type according to the methods described in section 4.7.2 Method of Combining Responses. Values along the highlighted diagonal are the probability that each management action, if recommended, would be implemented. The off-diagonal values per row represent the probabilities of each of the other alternative management actions being implemented instead of the recommended action. Probabilities across columns per row sum to 1, though this is not apparent in all cases shown due to rounding to two decimal places]

\section{A, Mixed-Grass}

\section{Implemented action}

\begin{tabular}{c|c|c|c|c|}
\cline { 2 - 5 } $\begin{array}{c}\text { Recommended } \\
\text { action }\end{array}$ & \multicolumn{1}{c}{ Rest } & \multicolumn{2}{c}{ Graze } & \multicolumn{2}{c}{ Burn } & Burn/Graze \\
\hline \multirow{2}{*}{$\begin{array}{c}\text { Rest } \\
\text { Graze }\end{array}$} & 0.9180 & 0.0613 & 0.0146 & 0.0061 \\
\cline { 2 - 5 } Burn & 0.0970 & 0.8107 & 0.0569 & 0.0354 \\
\cline { 2 - 5 } Burn/Graze & 0.1531 & 0.1771 & 0.6118 & 0.0580 \\
\cline { 2 - 5 } & 0.1218 & 0.2535 & 0.1348 & 0.4899 \\
\cline { 2 - 5 } & & & &
\end{tabular}


$B$, Tallgrass

\begin{tabular}{c|c|c|c|c|}
\multirow{2}{*}{$\begin{array}{c}\text { Recommended } \\
\text { action }\end{array}$} & Rest & \multicolumn{4}{c}{$\begin{array}{c}\text { Graze } \\
\text { within window }\end{array}$} & $\begin{array}{c}\text { Burn } \\
\text { within window }\end{array}$ & $\begin{array}{c}\text { Defoliate } \\
\text { outside window }\end{array}$ \\
\cline { 2 - 5 } Rest & 0.9622 & 0.0188 & 0.0001 & 0.0189 \\
\hline $\begin{array}{c}\text { Graze } \\
\text { within window }\end{array}$ & 0.1149 & 0.6049 & 0.0260 & 0.2542 \\
\hline $\begin{array}{c}\text { Burn } \\
\text { within window }\end{array}$ & 0.1122 & 0.1170 & 0.4805 & 0.2903 \\
\hline $\begin{array}{c}\text { Defoliate } \\
\text { outside window }\end{array}$ & 0.1468 & 0.1237 & 0.0807 & 0.6488 \\
\cline { 2 - 5 }
\end{tabular}

\subsubsection{Feasibility of Burning-A Special Case}

In addition to the reasons listed above (section 4.7 Partial Controllabilty) that can affect the ability to carry out an intended management action, such as unfavorable weather conditions and lack of resources, we also need to take into account the feasibility of carrying out management actions in consecutive management years. The Science Team acknowledged that the probability of carrying out a burn action on a unit depends on whether the unit was defoliated (e.g., grazed or burned) during the previous management year. This contingency revolves around whether or not the unit has sufficient fuel load to carry a fire. To account for the feasibility of burning, we developed a second set of matrices per grass type that reflects the partial controllability under the special circumstance of defoliation in the previous management year (that is, 'years since last defoliation' is equal to 1). We adjusted the mixedgrass matrix by decreasing the probability of implementing a burn action and a burn/graze action by 75 percent. We adjusted the tallgrass matrix by decreasing the probability of implementing a burn within window action by 25 percent. The specific choices for percent reductions were estimates supported by the Science Team. The probability that was taken from the burning actions was redistributed to the nonburning actions in proportion to their existing partition of the probability (table 14). The percent reductions for the two grass types differed because they took into account the different rates of vegetation growth after defoliation. 
Table 14. Partial controllability matrices for $A$, mixed-grass units and $B$, tallgrass units when the unit has been defoliated (that is, grazed or burned) in the previous management year.

A, Mixed-Grass

Implemented action

\begin{tabular}{|c|c|c|c|c|}
\hline $\begin{array}{c}\text { Recommended } \\
\text { action }\end{array}$ & Rest & Graze & Burn & Burn/Graze \\
\hline Rest & 0.9246 & 0.0702 & 0.0036 & 0.0015 \\
\hline Graze & 0.1254 & 0.8515 & 0.0142 & 0.0088 \\
\hline Burn & 0.3801 & 0.4525 & 0.1529 & 0.0145 \\
\hline Burn/Graze & 0.2880 & 0.5558 & 0.0337 & 0.1225 \\
\hline
\end{tabular}

$B$, Tallgrass

Implemented action

\begin{tabular}{c|c|c|c|c}
\cline { 2 - 5 } $\begin{array}{c}\text { Recommended } \\
\text { action }\end{array}$ & Rest & \multicolumn{1}{c}{$\begin{array}{c}\text { Graze } \\
\text { within window }\end{array}$} & \multicolumn{1}{c}{$\begin{array}{c}\text { Burn } \\
\text { within window }\end{array}$} & $\begin{array}{c}\text { Defoliate } \\
\text { outside window }\end{array}$ \\
\hline Rest & 0.9622 & 0.0188 & 0.0001 & 0.0189 \\
\hline $\begin{array}{c}\text { Graze } \\
\text { within window }\end{array}$ & 0.1157 & 0.6089 & 0.0195 & 0.2559 \\
\hline $\begin{array}{c}\text { Burn } \\
\text { within window }\end{array}$ & 0.1382 & 0.1440 & 0.3604 & 0.3574 \\
\hline $\begin{array}{c}\text { Defoliate } \\
\text { outside window }\end{array}$ & 0.1500 & 0.1265 & 0.0605 & 0.6630 \\
\cline { 2 - 5 }
\end{tabular}

The partial controllability matrices are used in the optimization procedure (see section 4.8 Optimization). Which set of matrices is called (that is, those in table 13 for no defoliation in the previous year or those in table 14 for defoliation in the previous year) depends on the current defoliation state of the unit. Recall that defoliation state includes the years since a unit was last defoliated (1, 2-4, $5+$ ) and the defoliation level (low, med, high) of the unit (table 9). When a unit is currently in defoliation state 1-4 (that is, defoliated 2 or more years ago), the first set of partial controllability matrices is used. The second set of partial controllability matrices is used when a unit is currently in defoliation state 5-7 (that is, defoliated 1 year ago).

\subsubsection{Management Restrictions}

Some units may have permanent or semi-permanent management restrictions. For example, lack of water availability may result in the inability to graze a particular unit, or proximity to a town may 
result in a burning ban on a particular unit. To accommodate such management restrictions, we created alternative versions of the partial controllability matrices presented in table 13 (tables 15 and 16). These alternative matrices were derived by simply removing the rows and columns containing the restricted action(s) from the original matrix and rescaling the values in the cells that remain to sum to 1 across the columns of each row. For either restriction (that is, 'No Graze' or 'No Burn'), the mixed-grass matrix is reduced to a $2 \times 2$ matrix, whereas the tallgrass matrix is reduced to a $3 \times 3$ matrix. We also created an alternative version of the partial controllability matrices presented in table 14 for units that were defoliated in the previous year (table 17); an alternative version is only shown for units with the 'No Graze' management restriction, because the matrices for units with the inability to burn remain unchanged by the management action in the previous year. We adjusted the matrices in table 15 to derive the table 17 matrices by decreasing the probability of implementing a burn action for mixed-grass and tallgrass units by 75 and 25 percent, respectively (see section 4.7.4 Feasibility of Burning for the motivation of these percent reductions).

Table 15. Partial controllability matrices for $A$, mixed-grass units and $B$, tallgrass units with 'No Graze' restrictions (that is, the inability to apply a graze to a unit).

A, Mixed-Grass

\section{Implemented action}

\begin{tabular}{c|c|c|}
\hline $\begin{array}{c}\text { Recommended } \\
\text { action }\end{array}$ & \multicolumn{1}{c}{ Rest } & Burn \\
\hline Rest & 0.9844 & 0.0156 \\
\cline { 2 - 3 } Burn & 0.2002 & 0.7998 \\
\cline { 3 - 3 }
\end{tabular}

$B$, Tallgrass

Implemented action

\begin{tabular}{c|c|c|c}
\hline $\begin{array}{c}\text { Recommended } \\
\text { action }\end{array}$ & Rest & \multicolumn{1}{c}{$\begin{array}{c}\text { Burn } \\
\text { within window }\end{array}$} & $\begin{array}{c}\text { Defoliate } \\
\text { outside window }\end{array}$ \\
\hline Rest & 0.9806 & 0.0001 & 0.0193 \\
\hline $\begin{array}{c}\text { Burn } \\
\text { within window }\end{array}$ & 0.1271 & 0.5442 & 0.3287 \\
\hline $\begin{array}{c}\text { Defoliate } \\
\text { outside window }\end{array}$ & 0.1676 & 0.0920 & 0.7404 \\
\cline { 3 - 4 }
\end{tabular}


Table 16. Partial controllability matrices for $A$, mixed-grass units and $B$, tallgrass units with 'No Burn' restrictions (that is, the inability to apply a burn to a unit).

A, Mixed-Grass

\section{Implemented action}

\begin{tabular}{c|c|c|}
\hline $\begin{array}{c}\text { Recommended } \\
\text { action }\end{array}$ & Rest & Graze \\
\hline \multirow{2}{*}{ Rest } & 0.9374 & 0.0626 \\
\cline { 2 - 3 } Graze & 0.1069 & 0.8931 \\
\cline { 2 - 3 } & &
\end{tabular}

$B$, Tallgrass

\section{Implemented action}

\begin{tabular}{c|c|c|c|}
\hline $\begin{array}{c}\text { Recommended } \\
\text { action }\end{array}$ & Rest & \multicolumn{1}{c}{$\begin{array}{c}\text { Graze } \\
\text { within window }\end{array}$} & \multicolumn{1}{c}{$\begin{array}{c}\text { Defoliate } \\
\text { outside window }\end{array}$} \\
\hline Rest & 0.9623 & 0.0188 & 0.0189 \\
\hline $\begin{array}{c}\text { Graze } \\
\text { within window }\end{array}$ & 0.1180 & 0.6210 & 0.2610 \\
\hline $\begin{array}{c}\text { Defoliate } \\
\text { outside window }\end{array}$ & 0.1597 & 0.1346 & 0.7057 \\
\cline { 2 - 4 } & & &
\end{tabular}

Table 17. Partial controllability matrices for $A$, mixed-grass units and $B$, tallgrass units with 'No Graze' restrictions when the unit has been defoliated (that is, burned) in the previous management year.

A, Mixed-Grass

\section{Implemented action}

\begin{tabular}{c|c|c|}
\hline $\begin{array}{c}\text { Recommended } \\
\text { action }\end{array}$ & Rest & Burn \\
\hline Rest & 0.9961 & 0.0039 \\
\cline { 2 - 3 } Burn & 0.8001 & 0.1999 \\
\cline { 2 - 3 }
\end{tabular}


$B$, Tallgrass

Implemented action

\begin{tabular}{c|c|c|c}
\hline $\begin{array}{c}\text { Recommended } \\
\text { action }\end{array}$ & Rest & \multicolumn{1}{c}{$\begin{array}{c}\text { Burn } \\
\text { within window }\end{array}$} & $\begin{array}{c}\text { Defoliate } \\
\text { outside window }\end{array}$ \\
\hline Rest & 0.9806 & 0.0001 & 0.0193 \\
\hline $\begin{array}{c}\text { Burn } \\
\text { within window }\end{array}$ & 0.1650 & 0.4081 & 0.4269 \\
\hline $\begin{array}{c}\text { Defoliate } \\
\text { outside window }\end{array}$ & 0.1718 & 0.0690 & 0.7592 \\
\cline { 2 - 4 }
\end{tabular}

\subsection{Optimization}

We used adaptive stochastic dynamic programming to compute optimal decisions that accounted for expected future behavior of the system from given current prairie conditions; that is, we computed state-dependent decision policies (Bellman, 1957; Anderson, 1975; Puterman, 1994; Lubow, 1995, 1997). The policies also accounted for structural uncertainty regarding the appropriate system model. We approached this accounting in two ways. For the mixed-grass type, we used an active adaptive optimization approach, in which the dynamics of future learning about those models was explicitly recognized; that is, we accounted for how learning was expected to take place in response to actions taken. For the tallgrass type, we used a passive adaptive optimization approach, in which structural uncertainty was recognized, but not the dynamics of future learning. We also took into account uncertainty with respect to randomness of native prairie composition outcome (environmental stochasticity) and uncertainty with respect to implementation of a recommended management action (partial controllability). We completed separate optimization analyses for mixed-grass and tallgrass types, using corresponding models and decision alternatives for each.

Here we describe the computational approach for active adaptive optimization (Williams, 1996; Moore and Conroy, 2006); a simplification yields the passive approach, which is described later in this section. Our goal was to discover the optimal sequence of actions $\boldsymbol{a}^{*}=\left\{a_{1}{ }^{*}, a_{2}{ }^{*}, \ldots, a_{T}^{*}\right\}$ over a long timeframe $t=1,2, \ldots, T$ that maximized the total expected utility returned from management, where the expectation is with respect to stochastic outcomes (due to the random environment and partial controllability) and to uncertainty about choice of model. For a sufficiently long timeframe, we hoped to find that the sequence of optimal actions became stationary; that is, the choice of the optimal action became dependent only on the current state and not time (Anderson, 1975). vector $\theta_{k}$ ):

We start by introducing notation for one of the predictive models (represented by parameter

$$
\operatorname{Pr}\left(S_{t+1}^{(j)} \mid S_{t}^{(i)}, a_{t+1}, \theta_{k}\right)=p_{k}\left(S_{t+1}^{(j)} \mid S_{t}^{(i)}, a_{t+1}\right) .
$$

In other words, $p_{k}$ represents the probability of transition from state $S^{(i)}$ at time $t$ to state $S^{(j)}$ at time $t+1$ under action $a_{t+1}$, as predicted by model $k$. Values of $p_{k}$ are found in the cells of the transition probability matrices for the different prediction models (digital appendixes $6 \mathrm{a}$ and $6 \mathrm{~b}$ ).

However, because of the imperfect connection between the intended action at time $t$ and the action that is ultimately actualized, we introduce another model component to express partial controllability as follows: 


$$
\operatorname{Pr}\left(a_{t+1}^{\prime} \mid a_{t+1}\right)=c\left(a_{t+1}^{\prime} \mid a_{t+1}\right)
$$

That is, some other action $a^{\prime}$ has some probability of being implemented when the intended action was $a$. These probability values are found in the cells of the partial controllability matrices described earlier (tables 13 and 14). We then combine these two probability models and describe the probability of transition in terms of the expectation over all possible actions given the intended action:

$$
\dot{p}_{k}\left(S_{t+1}^{(j)} \mid S_{t}^{(i)}, a_{t+1}\right)=\sum_{a^{\prime} \in A} p_{k}\left(S_{t+1}^{(j)} \mid S_{t}^{(i)}, a_{t+1}^{\prime}\right) c\left(a_{t+1}^{\prime} \mid a_{t+1}\right)
$$

Here, the expectation is taken over all possible values $a^{\prime}$ in the decision set $A$. In effect, corresponding values in the transition probability matrices are averaged over the different actions, where the averaging weights are the probabilities from the partial controllability model.

If the current allocation of belief weight among the $K$ models at time $t$ is $\pi_{t}=\left\{\pi_{t 1}, \pi_{t 2}, \ldots, \pi_{t K}\right\}$, then an expression of average transition probability with respect to model uncertainty and partial controllability is as follows:

$$
\bar{p}\left(S_{t+1}^{(j)} \mid S_{t}^{(i)}, a_{t+1}\right)=\sum_{k=1}^{K} \pi_{t k} \dot{p}_{k}\left(S_{t+1}^{(j)} \mid S_{t}^{(i)}, a_{t+1}\right)
$$

Next, we compute the expected single-step utility gained over all possible transitions out of state $S^{(i)}$ at time $t$ :

$$
\bar{v}\left(a_{t+1} \mid S_{t}^{(i)}, \pi_{t}\right)=\sum_{S_{t+1} \in S} \bar{p}\left(S_{t+1}^{(j)} \mid S_{t}^{(i)}, a_{t+1}\right) u\left(S_{t}^{(i)}, S_{t+1}^{(j)}, a_{t+1}\right)
$$

where $u\left(S_{t}^{(i)}, S_{t+1}^{(j)}, a_{t+1}\right)$ is the utility value corresponding to states $S_{t}^{(i)}$ and $S_{t+1}^{(j)}$ and action $a_{t+1}$. Quantity $\bar{v}\left(a_{t+1} \mid S_{t}^{(i)}, \pi_{t}\right)$ represents the average utility we should expect to realize (across models and across partially controlled actions) for action $a_{t+1}$ given that we currently observe state $S_{t}^{(i)}$ and currently allocate belief weight as $\pi_{t}$. Thus, $\bar{v}\left(a_{t+1} \mid S_{t}^{(i)}, \pi_{t}\right)$ is our expectation of utility after a single year of management.

Our aim is to find the sequence of actions that maximizes total expected utility over a specified timeframe. Therefore, we would like to find that action that maximizes the immediate return $\bar{v}\left(a_{t+1} \mid S_{t}^{(i)}, \pi_{t}\right)$ and those future actions that maximize total expected discounted utility over all future time periods $t+1, t+2$, and so forth. This goal can be expressed as a recurrence relationship (Bellman, 1957; Williams and others, 2002):

$$
\bar{V}^{*}\left(S_{t_{\mathrm{B}}}^{(i)}, \boldsymbol{\pi}_{t_{\mathrm{B}}}\right)=\operatorname{Max}_{a_{\mathrm{B}}+1}\left\{\bar{v}\left(a_{t_{\mathrm{B}}+1} \mid S_{t_{\mathrm{B}}}^{(i)}, \pi_{t_{\mathrm{B}}}\right)+\sum_{t=t_{\mathrm{B}}+1}^{T} \lambda^{t-1} \sum_{S_{t+1} \in S} \bar{p}\left(S_{t+1}^{(j)} \mid S_{t}^{(i)}, a_{t+1}\right) \bar{V}^{*}\left(S_{t+1}^{(i)}, \boldsymbol{\pi}_{t+1}\right)\right\},
$$

where $0 \leq \lambda \leq 1$ is an optional discounting factor that reflects the value at time $t$ of a return received at time $t+1$. A solution for the optimal sequence of actions $\boldsymbol{a}^{*}=\left\{a_{1}{ }^{*}, a_{2}{ }^{*}, \ldots, a_{T}^{*}\right\}$ over the timeframe is obtained by iterative application of this equation. The solution algorithm iterates backwards in time from an arbitrary endpoint, $T$. Starting at $T$, and then at each time point $t_{\mathrm{B}}$ preceding $T$, the solution algorithm finds a sequence of optimal actions $a\left(t_{\mathrm{B}}+1\right)^{*}, a\left(t_{\mathrm{B}}+2\right)^{*}, \ldots, a(T)^{*}$ for each possible combination of $S_{t}^{(i)}$ and $\pi_{t}$ that maximizes expected accumulated discounted utility over the interval, $\bar{V}^{*}\left(S_{t_{\mathrm{B}}}^{(i)}, \boldsymbol{\pi}_{t_{\mathrm{B}}}\right)$. The entire set of optimal actions over all native prairie states (defoliation states $\times$ vegetation states), all 
model belief states, and all time steps constitutes the full time-dependent decision policy. Iterations may continue in this manner over an ever-longer timeframe until the policy converges on a stationary solution; that is, until further steps backward in time do not elicit changes in the array of state-specific actions (Anderson, 1975). At this point, the decision policy requires no reference to time and can therefore be properly applied in the context of decision making over an indeterminate time horizon.

In the formulation above for active adaptive optimization (equations 13 and 14), the model belief weight vector $\pi_{t}$ is treated as a dynamic state that changes over time in response to management actions. Each action is evaluated on its expected contribution to long-term management performance by its influence on model belief weights (learning). An alternative treatment is to consider $\pi$ as a fixed (held constant) quantity through time; that is, we solve for the optimal policy under the assumption that model belief weights are insensitive to actions taken. Under this approach, model uncertainty is accounted for in the optimization, but not in a way that anticipates that learning can be acquired over time. In implementation of decision making, however, learning is in fact acquired as decisions are made and as monitoring outcomes are compared to model predictions. Each time learning is acquired and expressed in the form of new model belief weights, the decision policy is recomputed using the new vector of weights, which are again held fixed. The succession of decision policies produced under this approach are termed 'passive' (Johnson and others, 1997), because learning results as an unplanned byproduct of management, not as a prospective consideration of decision making. Although learning under a passive approach is slower than under an active approach, passive adaptive decision policies are far easier and faster to compute than active policies. Computation of the passive policy requires formulating the expected single-step utility as

$$
\bar{v}\left(a_{t+1} \mid S_{t}^{(i)}, \boldsymbol{\pi}\right)=\sum_{k=1}^{K} \pi_{k} \sum_{S_{t+1} \in S} \dot{p}_{k}\left(S_{t+1}^{(j)} \mid S_{t}^{(i)}, a_{t+1}\right) u\left(S_{t}^{(i)}, S_{t+1}^{(j)}, a_{t+1}\right)
$$

and the optimization equation as

$$
\bar{V}^{*}\left(S_{t_{\mathrm{B}}}^{(i)}, \boldsymbol{\pi}\right)=\operatorname{Max}_{a_{t_{\mathrm{B}}+1}}\left\{\bar{v}\left(a_{t_{\mathrm{B}}+1} \mid S_{t_{\mathrm{B}}}^{(i)}, \boldsymbol{\pi}\right)+\sum_{t=t_{\mathrm{B}}+1}^{T} \lambda^{t-1} \sum_{k=1}^{K} \pi_{k} \sum_{S_{t+1} \in S} \dot{p}_{k}\left(S_{t+1}^{(j)} \mid S_{t}^{(i)}, a_{t+1}\right) \bar{V}^{*}\left(S_{t+1}^{(i)}, \boldsymbol{\pi}\right)\right\} .
$$

We used Adpative Stochastic Dynamic Programming (ASDP; Lubow, 1995; 1997) to compute optimal stationary decision policies under uncertainty about vegetation dynamics (ASDP code in digital appendix 9a for mixed-grass and digital appendix $9 \mathrm{~b}$ for tallgrass). We used active adaptive optimization for mixed-grass prairie units (equations 13 and 14) and passive adaptive optimization for tallgrass prairie units (equations 15 and 16). We used the passive approach for tallgrass units because the number of models we considered under the tallgrass framework made active adaptive optimization impractical due to protracted computation time for the degree of solution precision we desired. In both the active and passive adaptive optimization, the ASDP program used transition models for defoliation state ( 7 state levels) and vegetation state (16 state levels) to account for state transitions in response to treatments. Each hypothesis (indexed by $k$ ) about vegetation dynamics was represented by a single predictive model [these models represented as $p_{k}\left(S_{t+1}^{(j)} \mid S_{t}^{(i)}, a_{t+1}\right)$ in equation 9]. For active optimization, the belief weight for each model at time $t$ was represented by $\pi_{t k}$ (equations 13 and 14), whereas for passive optimization (in which temporal dynamics of learning are ignored), the belief weight for each model was represented by $\pi_{k}$ (equations 15 and 16). The program also incorporated models of partial control over the management action [ $c\left(a_{t+1}^{\prime} \mid a_{t+1}\right)$ in equation 10]. With these components, the program integrated utilities that we assigned to transitions among native cover levels 
for different modes of treatment $\left[u\left(S_{t}^{(i)}, S_{t+1}{ }^{(j)}, a_{t+1}\right)\right.$ in equations 13 and 15]. Finally, we chose a discount value $\lambda$ of 0.993 , which translates into a reward received 100 years in the future as one-half as valuable as the same reward received today. We chose $\lambda<1$ for technical reasons to improve convergence to a stationary policy, but we chose a value large enough to maintain high value on expected future returns (see section 4.2.3 Objective Function).

We computed active adaptive decision policies for three scenarios under the mixed-grass framework: (1) no constraints regarding application of burning or grazing (tables 13A or 14A), (2) permanent inability to apply grazing in a unit (that is, rest or burn as the only possible decision options; tables $15 A$ or $17 A$ ), and (3) permanent inability to apply burning in a management unit (that is, rest or graze as the only possible decision options; table 16A). For each model, model belief weight was considered over the range $0-1$ in increments of 0.125 , which induced a model weight state space of 165 combinations of $\left\{\pi_{t 1}, \pi_{t 2}, \pi_{t 3}, \pi_{t 4}\right\}$. In combination with 7 levels of defoliation state and 16 levels of vegetation state, the decision policies under the mixed-grass framework provided an optimal action (rest, graze, burn, burn/graze) for each of 18,480 combinations of defoliation state, vegetation state, and model belief weight (digital appendix 10). For each scenario, we iterated the recursive optimization equation over a time horizon of $T=1,000$ years, and we considered the policy at the last iteration to be the stationary decision policy. The no-constraint scenario required 49 hours of computing time on a $3.73 \mathrm{GHz}$ Intel Xeon processor.

For the tallgrass framework, we computed optimal policies for the same three scenarios considered under the mixed-grass framework: (1) no constraints regarding the application of burning or grazing (tables $13 B$ or $14 B$ ), (2) permanent inability to apply grazing in a unit (that is, rest, burn within window, or defoliate as the only possible decision options; tables $15 B$ or $17 B$ ), and (3) permanent inability to apply burning in a unit (that is, rest, graze within window, or defoliate as the only possible decision options; table 16B). Lacking historical data to assess predictive ability of each model, we assigned equal belief weight (one-sixth) to each model in the optimization for the 2011 management year. Because model belief weights are not a part of the decision state space, the decision policies under the tallgrass framework provided an optimal action (rest, graze within window, burn within window, defoliate) for each of 112 combinations of defoliation state and vegetation state (see section 6.0 Implementation; fig. 12). For each scenario, we iterated policy computations over a 2,000-year time horizon, and we accepted the policy at the last iteration as the stationary (and nonadaptive) decision policy. Each scenario required $0.5-2$ hours of computing time.

\subsection{Monitoring}

A program of annual monitoring (June-August) on each management unit provides information about the unit's current vegetation state. This information is used to choose an appropriate action each year, to update belief weight assigned to each model, and to assess progress towards management goals. Indirectly, the information also becomes valuable over time for the construction or improvement of system models.

The monitoring design and protocols were developed by Grant and others (2004) (see digital appendix 11 for a copy of this paper). This approach, belt-transect monitoring, was designed to provide an unbiased and replicable assessment of vegetation composition in a manner that is efficient and robust to different observers. Belt-transect monitoring was implemented in a large-scale inventory of NWRS prairies throughout the Dakotas (Grant and others, 2009). Therefore, many cooperators in NPAM were familiar with this form of monitoring, and quickly agreed to adopt this monitoring design for recurrent use in NPAM. 
Each unit receives an allocation of permanent, 25-m linear transects at a target density of one transect per 3-5 acres (1.2-2.0 ha) depending on unit size, with a minimum of four transects. Transects, placed in random orientations, are located across the unit in a constrained random fashion, in which no transect is within $65 \mathrm{~m}$ of any other or within a 5-m spatial buffer of fences, structures, and permanent wet areas. Each transect is divided into 50 one-half-meter long segments. In each segment, the observer makes an ocular assessment of the dominant vegetation type in a $0.1-\mathrm{m}$ wide strip that is bisected by the transect line. The dominant vegetation type is chosen from a list of types developed for South Dakota units ('South Dakota plant list', appendix 7a) or for North Dakota, Montana, and Minnesota units ('North Dakota plant list', appendix 7b). Each monitored transect thus yields a list of no more than 50 encounters of one or more vegetation types (transects partially obscured by flooding may yield fewer than 50 encounters). A full description of the monitoring design and protocol is found in the NPAM Protocol Notebook (digital appendix 11).

For each transect, we classified each vegetation type into one of four vegetation classes: native prairie (NP), smooth brome (SB), Kentucky bluegrass (KB), and remainder (RM) (appendix 7a and 7b). We summed occurrences of each class into a vector of counts $y=\left\{n^{\mathrm{NP}}, n^{\mathrm{SB}}, n^{\mathrm{KB}}, n^{\mathrm{RM}}\right\}$ per transect.

\subsection{Model Weight Updating}

In the mixed-grass and tallgrass decision frameworks, each model is assigned a model belief weight that quantifies the influence of the model on the evaluation and selection of the current decision. The weights are treated as probabilities over a discrete set; therefore, their values are confined within the range $0-1$, and their sum across the model set is 1.0 . The belief weights are used in conjunction with vegetation and defoliation states to choose an optimal action (see section 4.8 Optimization). In an active adaptive decision policy, the belief weights are used as look-up values in the large decision table (digital appendix 10). In a passive adaptive decision policy, the belief weights are assumed to remain fixed through time, and the decision policy is re-computed each year on this basis.

The annual re-allocation of belief weight among models is the fundamental mechanism of adaptive management. To the extent that a model predicts an outcome well, the model inherits a greater share of belief weight for the next cycle of decision making. Conversely, a poorly predicting model loses belief weight and loses influence. Bayes' theorem is used to resolve a model's predictive performance with its prior belief to produce updated model belief weights (Williams 2001):

$$
w_{i, t+1}=\frac{f_{i}\left(s_{t+1} \mid s_{t}, a_{t+1}\right) w_{i, t}}{\sum f_{j}\left(s_{t+1} \mid s_{t}, a_{t+1}\right) w_{j, t}} .
$$

Here, $w_{i, t}$ and $w_{i, t+1}$ are the belief weights associated with model $i$ at times $t$ (before the action) and $t+1$ (following the action), respectively, and $f_{i}\left(s_{t+1} \mid s_{t}, a_{t+1}\right)$ is the likelihood of observing a transition from state $s_{t}$ to state $s_{t+1}$ in response to action $a_{t+1}$ under model $i$. For NPAM, $s_{t}=\left\{x_{t}, d_{t}\right\}$, that is, a specific combination of vegetation state $x_{t}=\{1,2, \ldots, 16\}$ and defoliation state $d_{t}=\{1,2, \ldots, 7\}$ for the management unit. The likelihood for model $i$ then can be written as follows:

$f_{i}\left(s_{t+1} \mid s_{t}, a_{t+1}\right)=f_{i}\left(\left\{x_{t+1}, d_{t+1}\right\} \mid\left\{x_{t}, d_{t}\right\}, a_{t+1}\right)$.

Because the transitions for vegetation and defoliation states are independent, the model likelihood can be expressed as follows:

$f_{i}\left(s_{t+1} \mid s_{t}, a_{t+1}\right)=T_{i}\left(x_{t+1} \mid\left\{x_{t}, d_{t}\right\}, a_{t+1}\right) q\left(d_{t+1} \mid d_{t}, a_{t+1}\right)$. 
The part of the model for the transition of the defoliation state, $q($.$) , is known (that is, a single$ model; see equation 5 and table 12); therefore, for purposes of model weight updating, $q($.$) , can be$ ignored. The vector of actions, $a_{t}=\{1,2,3,4\}$, represents rest, graze, burn, and burn/graze, respectively, for mixed-grass units, or rest, graze within window, burn within window, and defoliate, respectively, for tallgrass units.

If the true vegetation state of a site, $x_{t}$, could be observed, then the model-weight-updating calculations are straightforward. For $T_{i}\left(x_{t+1} \mid\left\{x_{t}, d_{t}\right\}, a_{t+1}\right)$, this requires extracting the appropriate element from the vegetation state transition probability matrix that corresponds to model $i$, action $a_{t+1}$, prior defoliation state $d_{t}$, prior vegetation state $x_{t}$, and current vegetation state $x_{t+1}$ (these matrices are found in digital appendix $6 \mathrm{a}$ and digital appendix $6 \mathrm{~b}$ ). Given values of the prior belief weight $w_{i, t}$, then values of $w_{i, t+1}$ are found easily through application of Bayes' theorem.

However, $x_{t}$ is observed indirectly from samples (transects) of the management unit (see section 4.9 Monitoring). For transects $j=1,2, \ldots, J$, a vector of observations is obtained, $\boldsymbol{y}_{j}=\left\{n_{j}^{\mathrm{NP}}, n_{j}^{\mathrm{SB}}, n_{j}^{\mathrm{KB}}\right.$, $\left.n_{j}{ }^{\mathrm{RM}}\right\}$, the counts of transect points dominated by native prairie, smooth brome, Kentucky bluegrass, and other vegetation, respectively $\left(0 \leq n_{j}^{(k)} \leq 50\right)$. An estimate of unit-level composition $\boldsymbol{p}=\left\{p^{\mathrm{NP}}, p^{\mathrm{SB}}, p^{\mathrm{KB}}\right.$, $\left.p^{\mathrm{RM}}\right\}$ can be obtained through the average of the counts for each vegetation type across all transects:

$$
\bar{n}^{(k)}=\sum_{j}^{J} n_{j}^{(k)} / J, \quad p^{(k)}=\bar{n}^{(k)} / \sum_{i}^{4} \bar{n}^{(i)} .
$$

If the $p^{(k)}$ are assumed fixed and measured without error, then they map into a single, fixed level of $x_{t}=$ $\{1, \ldots, 16\}$ through the following state assignment rules:

$$
\begin{aligned}
\text { np.state } & =\left\{\begin{array}{cc}
1: & 0.60 \leq p^{\mathrm{NP}} \\
2: & 0.45 \leq p^{\mathrm{NP}}<0.60 \\
3: & 0.30 \leq p^{\mathrm{NP}}<0.45 \\
4: & p^{\mathrm{NP}}<0.30
\end{array}\right. \\
\text { dom.state } & =\left\{\begin{array}{ccc}
1: & \frac{p^{\mathrm{RM}}}{1-p^{\mathrm{NP}}}<\frac{2}{3}, & \frac{p^{\mathrm{SB}}}{p^{\mathrm{SB}}+p^{\mathrm{KB}}} \geq \frac{2}{3} \\
2: & \frac{p^{\mathrm{RM}}}{1-p^{\mathrm{NP}}}<\frac{2}{3}, \frac{p^{\mathrm{SB}}}{p^{\mathrm{SB}}+p^{\mathrm{KB}}}<\frac{2}{3}, \quad \frac{p^{\mathrm{KB}}}{p^{\mathrm{SB}}+p^{\mathrm{KB}}}<\frac{2}{3} \\
3: & \frac{p^{\mathrm{RM}}}{1-p^{\mathrm{NP}}}<\frac{2}{3}, & \frac{p^{\mathrm{KB}}}{p^{\mathrm{SB}}+p^{\mathrm{KB}}} \geq \frac{2}{3} \\
4: & \frac{p^{\mathrm{RM}}}{1-p^{\mathrm{NP}}} \geq \frac{2}{3}
\end{array}\right.
\end{aligned}
$$

$x_{t}=4($ np.state -1$)+$ dom.state.

But because the $p^{(k)}$ are subject to sampling error, the assignment of vegetation state $x_{t}$ also is uncertain. We explicitly modeled the sampling process, allowing uncertainty to be incorporated in the estimation of unit-level composition $\boldsymbol{p}$, the resulting vegetation state $x_{t}$, and ultimately in the likelihood $T_{i}\left(x_{t+1} \mid\left\{x_{t}\right.\right.$, $\left.\left.d_{t}\right\}, a_{t+1}\right)$. In this approach, we assumed $\boldsymbol{p}$ to be a random quantity with density $\theta(\boldsymbol{\alpha})$; therefore, $x_{t}$ is also a random quantity with some categorical distribution. These processes can be represented in a hierarchical Bayesian model with three levels: unit level, transect level, and observation level (fig. 10). 


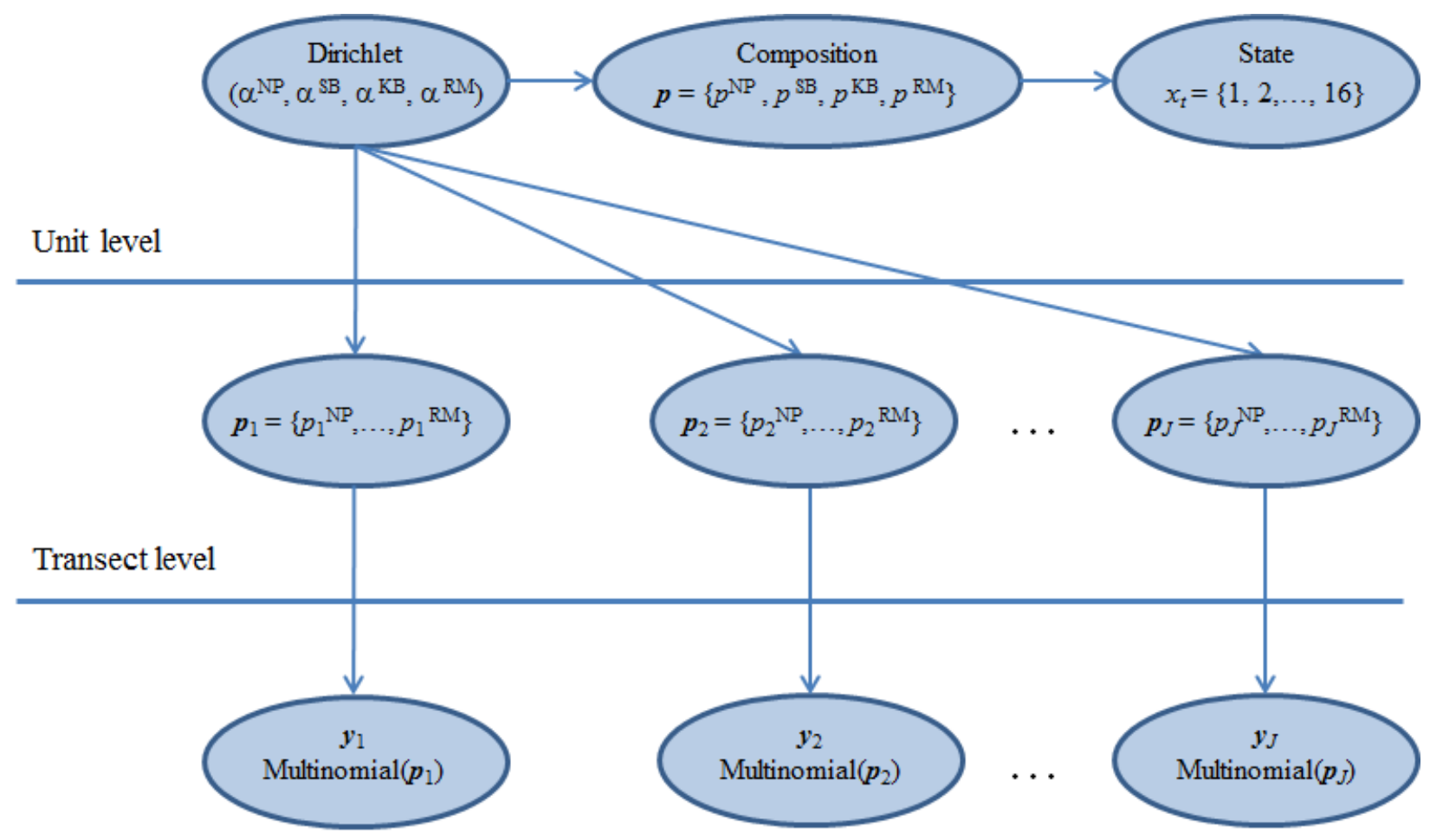

Observation level

Figure 10. Hierarchical Dirichlet-multinomial model for computing posterior distribution of vegetation state $x_{t}$ given a sample of $J$ transect-level observations $y_{j}=\left\{n_{j}^{\mathrm{NP}}, n_{j}^{\mathrm{SB}}, n_{j}^{\mathrm{KB}}, n_{j}^{\mathrm{RM}}\right\}$.

An appropriate model is the Dirichlet multinomial, the version of the beta-binomial for more than two categories (fig. 10). We assumed that the transect counts $\boldsymbol{y}_{j}=\left\{n_{j}^{\mathrm{NP}}, n_{j}^{\mathrm{SB}}, n_{j}^{\mathrm{KB}}, n_{j}^{\mathrm{RM}}\right\}$ comprised a multinomial draw of size $n_{j}=n_{j}^{\mathrm{NP}}+n_{j}^{\mathrm{SB}}+n_{j}^{\mathrm{KB}}+n_{j}^{\mathrm{RM}}$ for the success probabilities $\boldsymbol{p}_{j}=\left\{p_{j}^{\mathrm{NP}}, p_{j}^{\mathrm{SB}}\right.$, $\left.p_{j}^{\mathrm{KB}}, p_{j}^{\mathrm{RM}}\right\}$. We assumed that the vector $\boldsymbol{p}_{j}$ was itself random, arising as an outcome of a Dirichlet distribution with parameters $\alpha=\left\{\alpha^{\mathrm{NP}}, \alpha^{\mathrm{SB}}, \alpha^{\mathrm{KB}}, \alpha^{\mathrm{RM}}\right\}$. If $\alpha^{+}=\alpha^{\mathrm{NP}}+\alpha^{\mathrm{SB}}+\alpha^{\mathrm{KB}}+\alpha^{\mathrm{RM}}$, then the mean of this distribution is $\boldsymbol{p}=\left\{\alpha^{\mathrm{NP}} / \alpha^{+}, \alpha^{\mathrm{SB}} / \alpha^{+}, \alpha^{\mathrm{KB}} / \alpha^{+}, \alpha^{\mathrm{RM}} / \alpha^{+}\right\}$and represents the unit-level mean vegetation composition.

We used the Gibbs sampling algorithm (Link and Barker, 2010) to estimate the parameters of the model (W. A. Link, Patuxent Wildlife Research Center, written commun.). The conjugacy of the Dirichlet for the component proportion parameters allowed the transect-level $\boldsymbol{p}_{j}$ s to be updated in a straightforward manner. We updated the parameters of the Dirichlet distribution by sampling values of each parameter in turn from the likelihood, conditional on other parameters held at fixed values. For each unit and each year, we simulated 1,000,000 samples (after discarding 5,000 burn-in samples) to compute posterior distributions for the $\alpha$ parameters, for the unit-level component means $\boldsymbol{p}$, and for the unit-level vegetation state $x$ using the state assignment rule above.

By performing this analysis for a pair of successive years, we estimated the joint probability distribution for the state pair $\left(x_{t}, x_{t+1}\right)$ conditional on field observations in years $t$ and $t+1$. This distribution, $g\left(\left\{x_{t}, x_{t+1}\right\} \mid\left\{\boldsymbol{y}_{t}, \boldsymbol{y}_{t+1}\right\}\right)$, is defined over the $16 \times 16$ state space for $x_{t}$ and $x_{t+1}$, and it specifies the probability of each possible $\left(x_{t}, x_{t+1}\right)$ pair given that $\boldsymbol{y}_{t}$ and $\boldsymbol{y}_{t+1}$ were observed. From this 
distribution, we computed an expectation of likelihood for model $i$ with respect to the uncertain state pair $\left(x_{t}, x_{t+1}\right)$ :

$$
\bar{T}_{i}\left(\left\{x_{t}, x_{t+1}\right\} \mid d_{t}, a_{t+1}, \mathbf{y}_{t}, \mathbf{y}_{t+1}\right)=\sum_{x_{t}} \sum_{x_{t+1}} T_{i}\left(x_{t+1} \mid x_{t}, d_{t}, a_{t+1}\right) g\left(\left\{x_{t}, x_{t+1}\right\} \mid\left\{\mathbf{y}_{t}, \mathbf{y}_{t+1}\right\}\right)
$$

In other words, elements of the transition probability matrix for model $i$, defoliation state $d_{t}$, and action $a_{t+1}$ are averaged together in proportion to the probability of $\left(x_{t}, x_{t+1}\right)$.

Monitoring data were collected on mixed-grass and tallgrass sites in years 2009, 2010, 2011, and 2012. Thus, prior model weights established in 2009 potentially could be updated through three successive cycles of updating. For mixed-grass units, we computed model belief weights for 2011 based on two annual cycles of updating from a starting point of equal model weight $(w=1 / 4)$ assigned in 2009. Given the updated 2011 model belief weights, we carried out the third updating cycle to compute the 2012 model belief weights. For tallgrass units, the suite of management actions was restructured in 2011 and we lacked key data on how actions were carried out during the 2010 management year to characterize these actions in terms of the 2011 definitions. For this reason, model likelihoods could not be calculated for tallgrass data prior to 2010; therefore, we assigned equal model weights $(w=$ 1/6) to each of the tallgrass models in 2010 and, from this point, executed model weight updating for two successive cycles. We completed both updating cycles for tallgrass units in 2012.

We considered two approaches to conduct model weight updating for mixed-grass units. First, if management units are considered independent replicates of management action, then model weight updating can be accomplished by sequential updating over the collection of units within a single year. That is, if $\boldsymbol{w}_{\mathbf{0}}$ represents the prior set of model weights derived from year $t$, then a posterior set of weights $\boldsymbol{w}_{\mathbf{1}}$ may be obtained by application of Bayes' theorem to one management unit in year $t+1$. A second set of updated weights, $\boldsymbol{w}_{2}$, may be obtained in the same fashion by treating $\boldsymbol{w}_{\mathbf{1}}$ as prior weights for a second management unit. This process may be repeated until all $J$ management units are treated. The posterior weight thus produced, $\boldsymbol{w}_{\boldsymbol{J}}$, becomes the prior weight for application in the subsequent year, $t+1$.

However, given that the models were constructed largely on interpolations of expert opinion, and given our lack of experience with their predictions, we favored a more conservative updating approach that relied on an averaging of likelihoods across units. For each unit and model, we obtained the expected likelihood $\bar{T}_{i}\left(\left\{x_{t}, x_{t+1}\right\} \mid d_{t}, a_{t+1}, \mathbf{y}_{t}, \mathbf{y}_{t+1}\right)$ as described above, and we calculated the median value across units by model. We updated each year by applying the median likelihood values in Bayes' theorem (table 18). Compared to the sequential-unit approach, this approach had the benefit of tempering large shifts in model weight caused by chance observations that were not predicted well by any model. 
Table 18. A, Mixed-grass model weight updates for three cycles, 2009-10, 2010-11, and 2011-12, starting with equal model weights in 2009. B, Tallgrass model weight updates for two cycles, 2010-11 and 2011-12, starting with equal model weights in 2010.

[Annual model weights are shown in bold. Medians of the model-specific likelihoods are indicated for each annual update in regular font. The sample size of units (n) are indicated for each annual cycle]

$A$, Mixed-grass model weight updates

\begin{tabular}{llllll} 
Update cycle & & Model 1 & Model 2 & Model 3 & Model 4 \\
\hline & Prior weights 2009 & $\mathbf{0 . 2 5}$ & $\mathbf{0 . 2 5}$ & $\mathbf{0 . 2 5}$ & $\mathbf{0 . 2 5}$ \\
\hline $\begin{array}{l}2009-10 \\
(\mathrm{n}=66)\end{array}$ & Medians of model-specific likelihoods & 0.206067 & 0.169906 & 0.180916 & 0.18744 \\
\hline $\begin{array}{l}2010-11 \\
(\mathrm{n}=78)\end{array}$ & Updated weights 2010 & $\mathbf{0 . 2 7 6 8 4 9}$ & $\mathbf{0 . 2 2 8 2 6 8}$ & $\mathbf{0 . 2 4 3 0 5 9}$ & $\mathbf{0 . 2 5 1 8 2 4}$ \\
\hline $\begin{array}{l}\text { Up11-12 } \\
(\mathrm{n}=79)\end{array}$ & Medians of model-specific likelihoods & 0.171312 & 0.122149 & 0.132903 & 0.141037 \\
\hline
\end{tabular}

$B$, Tallgrass model weight updates

\begin{tabular}{llllllll} 
Update cycle & & Model 1 & Model 2 & Model 3 & Model 4 & Model 5 & Model 6 \\
\hline & Prior weights 2010 & $\mathbf{0 . 1 6 6 6 7}$ & $\mathbf{0 . 1 6 6 6 7}$ & $\mathbf{0 . 1 6 6 6 7}$ & $\mathbf{0 . 1 6 6 6 7}$ & $\mathbf{0 . 1 6 6 6 7}$ & $\mathbf{0 . 1 6 6 6 7}$ \\
\hline $\begin{array}{l}2010-11 \\
(\mathrm{n}=32)\end{array}$ & Medians of model-specific likelihoods & 0.21041 & 0.17415 & 0.19640 & 0.20364 & 0.19645 & 0.19533 \\
& Updated weights 2011 & $\mathbf{0 . 1 7 8 8 7}$ & $\mathbf{0 . 1 4 8 0 4}$ & $\mathbf{0 . 1 6 6 9 5}$ & $\mathbf{0 . 1 7 3 1 1}$ & $\mathbf{0 . 1 6 6 9 9}$ & $\mathbf{0 . 1 6 6 0 4}$ \\
\hline $\begin{array}{l}2011-12 \\
(\mathrm{n}=32)\end{array}$ & Medians of model-specific likelihoods & 0.24794 & 0.19628 & 0.23364 & 0.25906 & 0.23369 & 0.24451 \\
& Updated weights 2012 & $\mathbf{0 . 1 8 7 2 2}$ & $\mathbf{0 . 1 2 2 6 7}$ & $\mathbf{0 . 1 6 4 6 7}$ & $\mathbf{0 . 1 8 9 3 1}$ & $\mathbf{0 . 1 6 4 7 4}$ & $\mathbf{0 . 1 7 1 3 9}$ \\
\hline
\end{tabular}

The Gibbs sampler, the computation of $\bar{T}_{i}\left(\left\{x_{t}, x_{t+1}\right\} \mid d_{t}, a_{t+1}, \mathbf{y}_{t}, \mathbf{y}_{t+1}\right)$, and the model weight updating procedures are contained in a SAS program (digital appendix 12). At present (2013), prior model weights and monitoring data are supplied to the SAS program by way of queries run in an Access database (see section 4.12 Database), and the program returns model weight output in an Accessreadable data table. Ultimately, these procedures will be written in an executable language that can be called directly from Access, without user intervention and interaction with SAS (see section 7.2.1 NearTerm).

\subsection{Administration}

The Fish and Wildlife Service has in place two critical roles for the coordination and implementation of NPAM: the project coordinator and the database coordinator. The project coordinator oversees the operation of NPAM and orchestrates the annual sequence of tasks that 
comprise the framework. The project coordinator addresses the needs and concerns of the cooperators and assures that protocols are understood and followed. Major duties of the project coordinator include communication and outreach, coordination and oversight, training and support, and data management. The project coordinator works closely with and guides NPAM-related work of the database coordinator. The database coordinator provides effective oversight and maintenance of the highly interrelated data management system and geospatial tasks related to NPAM. Major duties of the database coordinator include data management, data aggregation and assembly, and technical support. Details of the roles of the project coordinator and database coordinator are found in the Protocol Notebook (digital appendix $11)$.

\subsection{Database}

The data collection, organization, and analytical needs of NPAM are supported by a localized Microsoft Access database (DB) available to the project coordinator and by a centralized Microsoft InfoPath application (IP) available to the project cooperators through the web-based Microsoft SharePoint collaboration platform. The cooperators enter information on management actions and monitoring data through the IP tool. The data are captured in the DB through a connection to SharePoint, and the project coordinator uses the DB to run consistency checks of the data, to summarize the data into useful tables, to perform model weight updating, and to generate recommended management actions for distribution to cooperators.

Prior to 2010, cooperators entered monitoring data into Excel spreadsheet templates. A general description of the management action was recorded (that is, 'burned', 'grazed', etc.), but details about the action were not recorded in any consistent fashion. Kevin McAbee (FWS) authored the first version of the DB, which contained screens to record attributes about the management action and to record vegetation monitoring data. This version was distributed to cooperators in 2010 for use as a stand-alone data entry platform. In 2011, Sarah Jacobi (Chicago Botanic Garden) made design and operational improvements to the DB. This improved version of the DB was again distributed to cooperators in 2011 for use in a stand-alone fashion. In 2012, Sarah Jacobi added functionality to the DB to perform required analyses (data consistency checks, action and state assignments, model weight updating, optimal decision lookup). These changes were designed to transition the DB from a data entry platform into a localized project management tool for use by the NPAM coordinator. A full description of the final DB is described in digital appendix 2. The final DB performs several primary functions, executed automatically and in sequence:

(1) Link with the SharePoint site to download and store cooperator-entered management and monitoring records (see subsequent paragraph);

(2) Use a set of rules to flag questionable management records for review by the coordinator;

(3) Assign each unit to an NPAM management action based on a rule set that interprets the management data;

(4) Assign each unit into a current defoliation and vegetation state based on rule sets applied to the management and vegetation monitoring data;

(5) Perform updating of model belief weights [currently (2013), prepares tables for input into SAS program];

(6) Select an optimal management action for each unit based on current states, updated model belief weights, and updated policies;

(7) Prepare reports for cooperators, including a unit-level summary of data and management recommendation. 
Stand-alone platforms for data entry were cumbersome to administer and introduced risks of data loss and contamination. In 2011, Vicky Hunt (Chicago Botanic Garden) began work on a SharePoint-hosted InfoPath interface as a replacement for stand-alone platforms. The data entry features of the DB (e.g., context-sensitive menus, pull-down options, auto-fill functions, validation and logical checks) were reproduced in the IP interface. Under this system, cooperators log in to SharePoint, and they are permitted read/write access to data for management units under their administration. As the cooperators' data are entered, they are instantly captured by IP, reducing risk of data loss. The system also has automated utilities that cooperators may run to produce unit-level data summaries. At the annual data entry deadline date (August 25), the project coordinator runs an automated process that establishes a link between the DB and SharePoint and downloads the IP records into the DB. In the 2012 field season, data entry by cooperators was completed in IP. A full description of the IP tool is described in digital appendix 13.

\subsection{Protocol Notebook}

We developed a comprehensive document, the Protocol Notebook, to describe to various audiences how the NPAM project is structured and how it is executed (digital appendix 11). The primary audience includes the project coordinator, the database coordinator, and the individual NPAM cooperators. For this audience, the Protocol Notebook is an operational reference manual, providing detailed guidance on how to establish management units, how to collect and enter data, how to characterize treatments, how to run the database tool, and many other required processes. Secondary audiences include the project's Advisory Team, FWS program managers, and other interested parties who desire an overall perspective of the project, including its general design, governance structure, and flow of operations.

The motivation for the Protocol Notebook stems from the recognition that cooperators differ in how they implement management, their degree of success in achieving a targeted action, and the range of uncontrollable environmental and logistic complications they face. To maximize the utility of the decision framework, these sources of variance should be minimized to the degree possible, and actions and outcomes should be recorded in sufficient detail to understand their consequences. The Protocol Notebook is intended to provide a set of clear, consistent guidelines for collecting data and applying management. It also will provide continuity for new staff when there is turnover.

The Protocol Notebook is laid out in three main sections. The first section is a general overview of the NPAM decision framework, including descriptions of the roles and responsibilities of the participants, definition of treatment alternatives, and guidance for participating and withdrawing from the NPAM program. The second section describes the field and GIS activities needed to prepare a management unit for participation in the program. The third section describes the recurrent activities related to applying management actions, collecting monitoring data, and computer entry of data for each unit. A set of appendixes provides a project record (a timeline of project development history), monitoring data sheets, user guides, plant lists, key literature, and other supporting documentation.

The Protocol Notebook is intended to be continually revised as the program evolves and modifications are implemented. Thus, the document captures program reforms that emerge from institutional learning exercises (see section 7.0 Institutional Learning). 


\subsection{Iterative Phase}

\subsection{Annual Cycle}

The iterative phase includes the recurrent steps that are repeated each year as part of the annual iterative adaptive management cycle (fig. 11). These recurrent steps include:

(1) The project coordinator provides the recommended management action per unit to refuge managers; the recommendation is based on the current vegetation state and defoliation state of each unit, the current knowledge state (that is, weight on each alternative model) of NPAM for each grass type, and the current optimal decision policy (the table of recommended optimal management actions, indexed by vegetation state and defoliation state-see section 4.8 Optimization) for each grass type.

(2) The managers make a management decision per unit, taking the recommendation into consideration, and implement the action during the management year. They record the action that was implemented, as well as the details of its implementation.

(3) The alternative models predict the consequences of each implemented action per unit.

(4) Refuge personnel monitor the resulting vegetation state on each unit.

(5) We assess the performance of the competing models by comparing the model-predicted outcomes per unit to the observed outcomes per unit and calculating model likelihoods. Weights on the alternative models are updated based on the unit-specific performance of each model.

(6) Repeat steps 1-5 the subsequent management year.

Steps 1 through 4 take place at the level of the individual management unit. Step 5 involves data from all units across a given grass-type of the whole NPAM region. Step 5 is the last step of the annual iterative cycle, and it is by this step that we obtain the new knowledge state and identify the optimal decision policy for the next management year. The current optimal decision policy, along with the current state of each management unit, is the basis for the recommended management action made in step 1 of the new iterative cycle. In short, data from individual management units are provided annually, these data are used to reduce uncertainty across the whole region, and updated annual decision guidance that incorporates understanding gained from the collective experience of all NPAM cooperators is delivered to individual managers. 


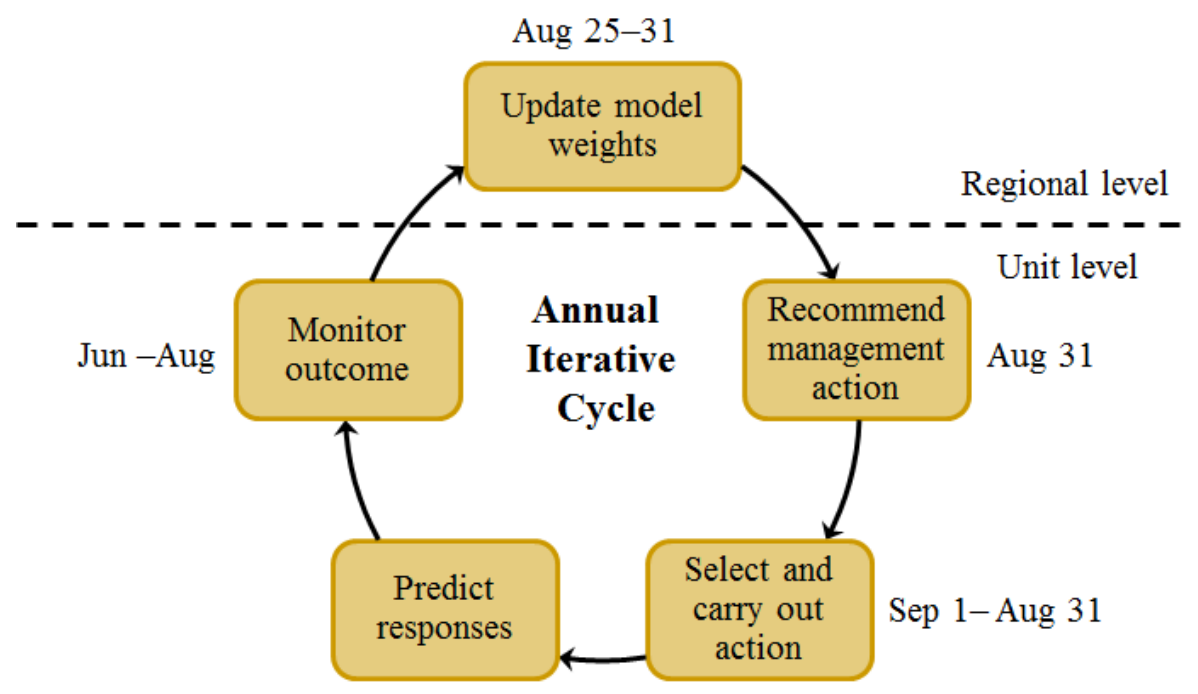

Figure 11. The annual iterative cycle includes five steps in sequence: (1) recommend a management action per unit; (2) select and carry out a management action per unit; (3) predict the vegetation response per unit under each alternative model; (4) monitor the vegetation response per unit; and (5) assess performance of alternative models and update their weight based on their performance. Steps 1 through 4 take place at the level of the individual management unit. Step 5 involves data from all units across a given grass-type of the whole Native Prairie Adaptive Management (NPAM) region.

\subsection{The Result of the Iterative Cycle}

It is through the steps of the annual iterative cycle that we reduce uncertainty about how the system responds to management; reduction of uncertainty is achieved by way of updating our confidence in each of the alternative models and distinguishing better models from poorer models. Models that perform better garner more weight at the expense of the models that perform less well. The shift in model weights embodies our learning and the reduction in our uncertainty about system behavior. Models with more weight exert greater influence on the next management decision through the updated optimal decision policy. The change in the decision policy exemplifies how management recommendations adapt based on our learning. Because future management decisions are based on recommendations that stem from an improved understanding of system behavior, the result is better management decisions than when we started. The starting model weights, updated model weights, and decision policies used each year in NPAM to date are documented in section 6.0 Implementation.

\subsection{Annual Data Processing Steps}

Cooperators enter all monitoring and management data in the web-based Microsoft SharePoint cooperator database by August 25 of each year. Between August 25 and August 31, the data are processed, the weights on the alternative models are updated, management recommendations for the next management year are determined, and summaries of data are completed. We have automated the annual data processing procedure within the Coordinator Database such that the project coordinator can complete it by performing the following automated steps:

(1) Based on rules we defined, the database classifies each individual management action (there may be more than one activity per unit per year) as one of the four recognized alternative actions 
(specific to grass type). It also flags actions that include cooperator comments or do not meet rules of consistency or minimum criteria.

(2) The coordinator must review all flagged actions and manually indicate that the review was completed. The coordinator also must review actions that were not classifiable as one of the four recognized alternative actions and has the option of manually reclassifying the action to one of the four alternative actions.

(3) The database next attempts to resolve multiple activities into a single management action per unit. Based on rules we defined, the database classifies the management action per unit as one of the four recognized alternative actions (specific to grass type). These classifications are based on rules of allowable combinations of multiple actions per unit within a single management year. Combinations that violate the rules are tagged as unclassifiable.

(4) The coordinator reviews units with unclassifiable management actions and has the option of manually reclassifying the unit to one of the four alternative actions. Units that remain unclassified are omitted from model weight updating.

(5) The database prepares the data required as input for the model weight updating program. These data include the following:

a. Vegetation monitoring data for all units for two consecutive years (years $t$ and $t+1$ )

b. Management action per unit for year $t+1$

c. Defoliation history per unit for seven-year window (that is, years $t-5$ to $t+1$ )

d. Current model weights

(6) The updating program, written in SAS, imports the data from step 5, processes the data, and exports the updated model weights back to the Access database. This step is external to the database (see section 7.2.1 Institutional Learning | Future Improvements/Modifications | NearTerm for plans to incorporate this step directly within the database).

(7) Given the updated model weights, the database selects the current decision policy from the optimization decision table and matches the recommended management action to each unit based on the unit's current vegetation and defoliation state.

(8) The database generates reports and data summaries to be provided to the cooperators. These reports include the vegetation and defoliation state, the recommended management action for the coming management year, as well as other summary information of interest to cooperators.

The Coordinator Database annual data processing steps are detailed in the Coordinator Database User Guide (digital appendix 2). As noted above, the updating procedure is currently (2013) run outside of the Access Coordinator Database. Planned future work includes writing the updating routine in an executable language $(\mathrm{C}++)$ that can be called internally by Access. In this manner, the entire data processing procedure will be fully automated within the Access Coordinator Database.

\subsection{Implementation}

From project inception (July 2008) through the 2012 decision cycle (August 2012), each annual cycle was an opportunity for prototyping; each year was in a sense a pilot year as we implemented, evaluated, and later modified framework components. Below we outline the implementation of the NPAM framework throughout the three annual cycles. 


\subsection{Summary of 2008-09}

- Recruitment of cooperators by means of a cooperator interest survey whereby cooperators nominated management units to enroll and participate in NPAM.

- Pilot season

○ Managers selected management actions per convention.

- Belt-transect vegetation monitoring was completed during the summer of 2009.

- Started development of the first prototype of the NPAM database

- Started development of the first prototype of the NPAM decision framework

\subsection{Summary of $2009-10$}

- Held $2^{\text {nd }}$ annual cooperator meeting in Jamestown, North Dakota (October 2009)

- Discussed the pilot season

- Demonstrated the first draft of the NPAM database

○ Demonstrated the first draft of the decision framework models

- Solicited cooperator feedback

- Cooperators enrolled 120 management units.

○ 81 mixed-grass units

- 13.6 acres $(\mathrm{ac})-594.8 \mathrm{ac} ;$ mean $=94 \mathrm{ac}$, standard deviation $(\mathrm{stdev})=82.2$

- 39 tallgrass units

- $7.7 \mathrm{ac}-197.1 \mathrm{ac} ;$ mean $=64.3 \mathrm{ac}, \mathrm{stdev}=43.8$

- Completed the first version of the NPAM database

- Pre-adaptive season

- Managers selected management actions per convention.

- Belt-transect vegetation monitoring was carried out during the summer of 2010.

- Completed the provisional adaptive management decision framework (appendix 2)

- Cooperators entered management and vegetation data into the new NPAM database for the 2009 and 2010 management years.

- First round of management guidance was provided; management actions for the 2011 management year were recommended to cooperators on August 31, 2010 (appendixes 8a and $8 b)$.

- Recommendations were based on the provisional decision framework (appendix 2) and the current state of each management unit (per 2010 monitoring).

- Alternative models were equally weighted.

\subsection{Summary of $2010-11$}

- Held 3rd annual cooperator meeting in Jamestown, North Dakota (February 2011)

- Reviewed the 2009 and 2010 vegetation and management data, cooperator feedback survey, three-year plan, accomplishments to date, what remains to be completed, components of the provisional decision framework, and existing and future modifications to framework elements

- Solicited cooperator feedback

- Cooperators enrolled 122 management units.

○ 84 mixed-grass units 
- $13.6 \mathrm{ac}-594.8 \mathrm{ac} ;$ mean $=94.3 \mathrm{ac}, \mathrm{stdev}=81.1$

○ 38 tallgrass units

- $7.7 \mathrm{ac}-197.1 \mathrm{ac} ;$ mean $=62.8 \mathrm{ac}$, stdev $=42.5$

- Cami Dixon, Region 6 Dakota Zone Biologist, joined the Science Team as the NPAM Project Coordinator.

- Jennifer Zorn, I\&M GIS Database Manager, joined the Science Team as the NPAM Database Coordinator.

- Reviewed and revised the elements of the provisional decision framework (first instance of 'double-loop learning')

- Contracted with Eric Lonsdorf, Sarah Jacobi, and Victoria Hunt to improve the existing NPAM database and to develop a centralized web-version of the database using the SharePoint platform

- First adaptive season

- Managers selected management actions after taking the 2011 recommended management actions into consideration.

- Belt-transect vegetation monitoring was carried out during the summer of 2011.

- Completed the updated adaptive management decision framework (see appendix 2 for details on the changes that were made to the provisional framework)

- Completed the updates to the existing NPAM database

- Cooperators entered management and vegetation data into the updated NPAM database for the 2011 management year.

- Second round of management guidance was provided; management actions for the 2012 management year were recommended to cooperators on August 31, 2011 (appendixes 8a and $8 b)$.

- Recommendations were based on the updated decision framework and the current state of each management unit (per 2011 monitoring).

- Mixed-Grass

- Completed two updating cycles (fig. $12 A$ ) and weighted alternative models according to relative credibility (table 19A)

- Optimal decision policy (fig. 13) was based on an active adaptive optimization procedure and the updated model weights (table 19A).

- Tallgrass

- Alternative models were weighted equally.

- Optimal decision policy (fig. 14) was based on a passive optimization procedure and equal model weights (table 19B).

\subsection{Summary of 2011-12}

- Held the hand-off meeting, 4th annual cooperator meeting in Jamestown, North Dakota (November 2011)

- Reviewed the 2011 vegetation and management data, three-year plan, accomplishments to date, ancillary studies, components of the updated decision framework, new centralized database, cooperator resources, tallgrass phenology, and implementation phase post hand-off

- Cooperators enrolled 118 management units.

- 82 mixed-grass units

- 13.6 ac -594.8 ac; mean $=93.8$ ac, stdev $=81.5$ 
○ 36 tallgrass units

- $7.7 \mathrm{ac}-143.6 \mathrm{ac}$; mean=59.2 ac, stdev=36.6

- Completed development of the centralized InfoPath application of the NPAM database on the web-based Microsoft SharePoint collaboration platform; the centralized database is for use by the cooperators to enter annual management and vegetation data

- Completed development of the localized Access database for use by the project coordinator to annually process cooperator data

- Second adaptive season

- Managers selected management actions after taking the 2012 recommended management actions into consideration.

- Belt-transect vegetation monitoring was completed during the summer of 2012.

- Cooperators entered management and vegetation data into the centralized database for the 2012 management year.

- The project coordinator used the localized database to process the newly entered 2012 data.

- Third round of management guidance was provided; management actions for the 2013 management year were recommended to cooperators on August 31, 2012 (appendixes 8a and $8 b)$.

- Recommendations were based on the decision framework and the current state of each management unit (per 2012 monitoring).

- Mixed-Grass

- Completed a third updating cycle (fig. 12A) and weighted alternative models according to relative credibility (table 19A)

- Optimal decision policy (fig. 13) was based on an active adaptive optimization procedure and the updated model weights (table 19A).

- Tallgrass

- Completed two updating cycles (fig. 12B) and weighted alternative models according to relative credibility (table 19B)

- Optimal decision policy (fig. 15) was based on a passive optimization procedure and the updated model weights (table 19B). 
$A$, Mixed-grass updating cycles

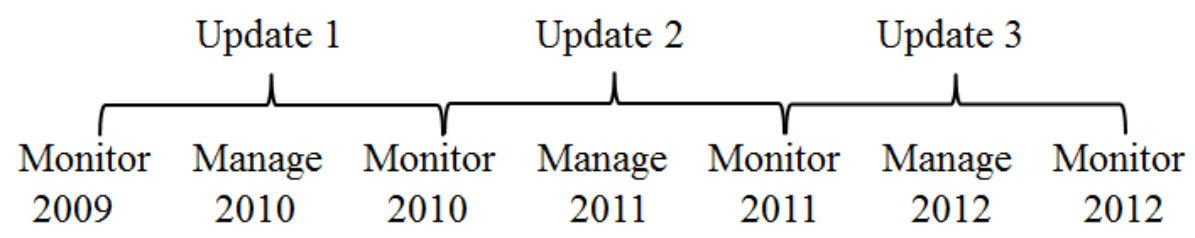

$B$, Tallgrass updating cycles

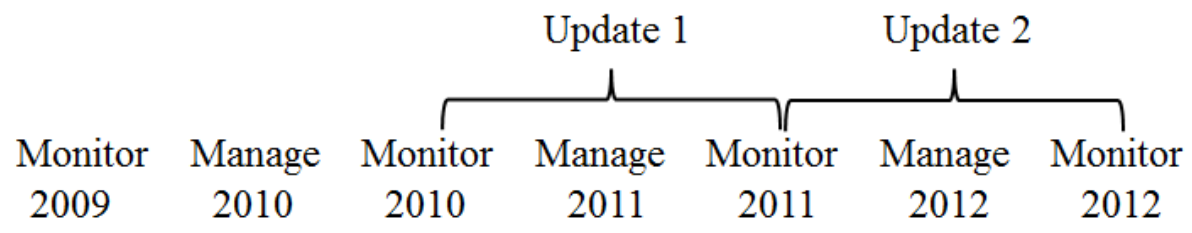

Figure 12. A, In August 2011, we completed two model weight updating cycles for the mixed-grass model set. The first update used paired monitoring data from 66 units observed in 2009 and 2010, along with the intervening management on the units. The second update used paired monitoring data from 78 units observed in 2010 and 2011, along with the intervening management. In August 2012, we completed a third updating cycle; the third update used paired monitoring data from 79 units observed in 2011 and 2012, along with the intervening management. Results of the mixed-grass updating cycles are shown in table 19A. B, In August 2012, we completed two model weight updating cycles for the tallgrass model set. The first update used paired monitoring data from 32 units observed in 2010 and 2011, along with the intervening management. The second update used paired monitoring data from 32 units observed in 2011 and 2012, along with the intervening management on the units. Results of the tallgrass updating cycles are shown in table 19B. We did not update with the paired 2009 and 2010 tallgrass data because the suite of management actions for tallgrass units was re-structured in 2011, and we lacked key data on how actions were carried out in 2010 to characterize them in terms of the 2011 definitions. 
Table 19. A, Results of the three model weight updating cycles for the mixed-grass model set.

[The first two updating cycles were completed in 2011, the first year that empirically derived model weights were used for Native Prairie Adaptive Management (NPAM) mixed-grass sites, through retrospective analysis of 2009 and 2010 data. Starting with equal weight on the four models in 2009, we sequentially updated the weights for the year 2010 and then the year 2011. Although we show the resulting updated model weights per updating cycle, empirically derived model weights were not used to identify a decision policy prior to 2011. The 2011 updated model weights represent the 2011 knowledge state, which we used to identify the 2011 optimal decision policy (fig. 13). The third updating cycle was completed in 2012. The 2012 updated model weights represent the 2012 knowledge state, which we used to identify the 2012 optimal decision policy (fig. 13)]

\begin{tabular}{l|c|c|c|c|}
\multicolumn{1}{c}{} & Model & Model & Model & Model \\
$\mathbf{1}$ & $\mathbf{2}$ & $\mathbf{3}$ & $\mathbf{4}$ \\
\cline { 2 - 5 } $\mathbf{2 0 0 9}$ & 0.25 & 0.25 & 0.25 & 0.25 \\
\cline { 2 - 5 } $\mathbf{2 0 1 0}$ & 0.277 & 0.228 & 0.243 & 0.252 \\
\cline { 2 - 5 } $\mathbf{2 0 1 1}$ & 0.331 & 0.195 & 0.226 & 0.248 \\
\cline { 2 - 5 } $\mathbf{2 0 1 2}$ & 0.375 & 0.164 & 0.208 & 0.253 \\
\cline { 2 - 5 }
\end{tabular}

$B$, Results of the two model weight updating cycles for the tallgrass model set.

[Both updating cycles were completed in 2012, the first year that empirically derived model weights were used for NPAM tallgrass sites, through retrospective analysis of 2010 and 2011 data. Starting with equal weight on the six models in the year 2010, we sequentially updated the model weights for the year 2011 and then the year 2012. Although we show the resulting updated model weights per updating cycle, empirically derived model weights were not used to identify a decision policy prior to 2012. In 2011, we assumed all models were equally credible, and we assigned equal model weights to identify the 2011 optimal decision policy (fig. 14). The 2012 updated model weights represent the 2012 knowledge state, which we used to identify the 2012 optimal decision policy (fig. 15). We did not update with the paired 2009 and 2010 tallgrass data because the suite of management actions for tallgrass units was re-structured in 2011, and we lacked key data on how actions were carried out in 2010 to characterize them in terms of the 2011 definitions]

\begin{tabular}{l|c|c|c|c|c|c|}
\multicolumn{2}{c}{$\begin{array}{c}\text { Model } \\
\mathbf{1}\end{array}$} & $\begin{array}{c}\text { Model } \\
\mathbf{2}\end{array}$ & $\begin{array}{c}\text { Model } \\
\mathbf{3}\end{array}$ & $\begin{array}{c}\text { Model } \\
\mathbf{4}\end{array}$ & $\begin{array}{c}\text { Model } \\
\mathbf{5}\end{array}$ & $\begin{array}{c}\text { Model } \\
\mathbf{6}\end{array}$ \\
\cline { 2 - 7 } $\mathbf{2 0 0 9}$ & - & - & - & - & - & - \\
\cline { 2 - 7 } $\mathbf{2 0 1 0}$ & 0.167 & 0.167 & 0.167 & 0.167 & 0.167 & 0.167 \\
\cline { 2 - 7 } $\mathbf{2 0 1 1}$ & 0.179 & 0.148 & 0.167 & 0.173 & 0.167 & 0.166 \\
\cline { 2 - 7 } $\mathbf{2 0 1 2}$ & 0.187 & 0.123 & 0.165 & 0.189 & 0.165 & 0.171 \\
\cline { 2 - 7 } & & & & & &
\end{tabular}


$A$, No management restrictions

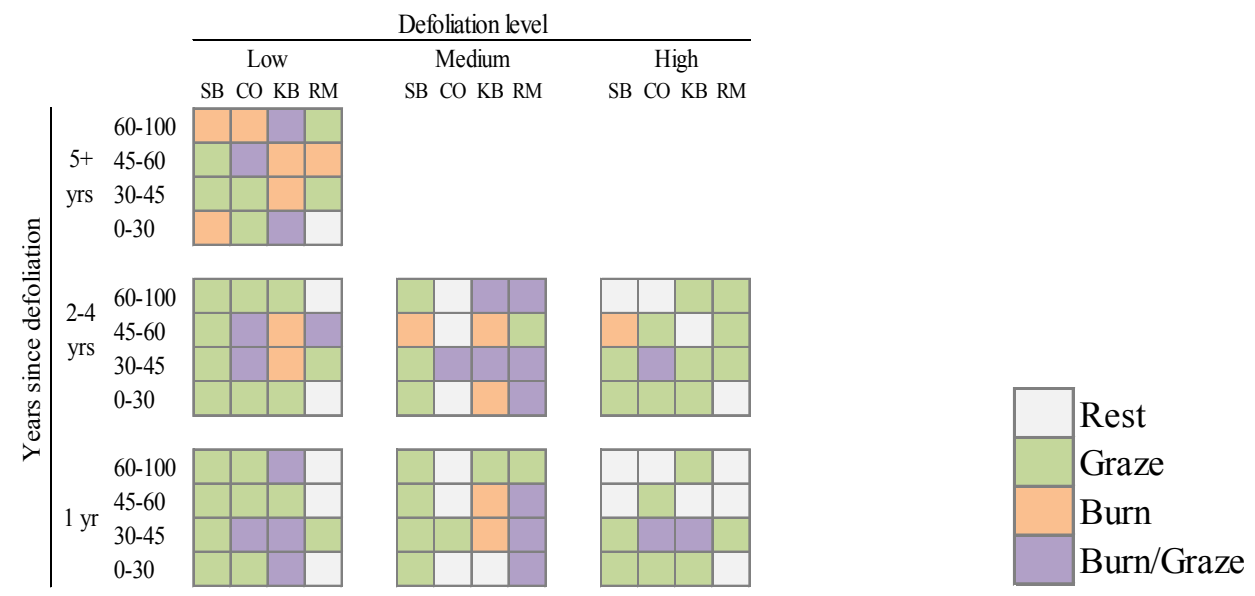

B, No graze, No burn/graze

C, No burn, No burn/graze
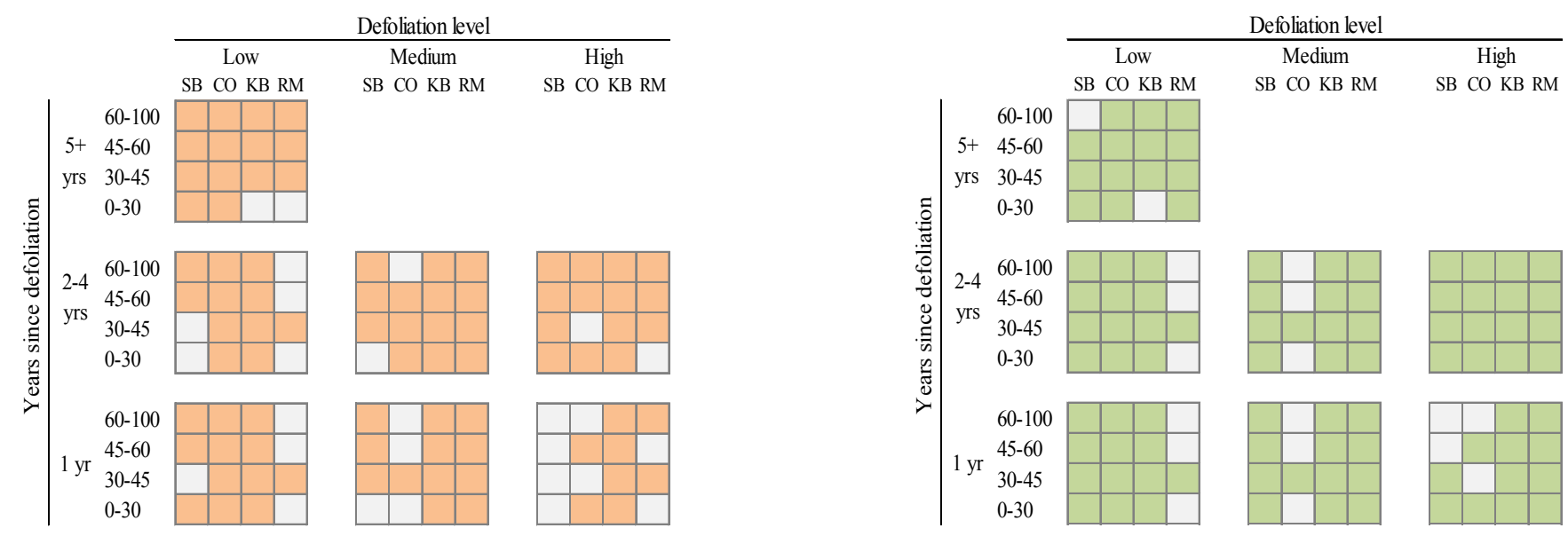

Figure 13. Mixed-grass 2011 and 2012 optimal decision policies for units with the following management action restrictions: $A$, no restrictions, $B$, cannot graze or burn/graze, and $C$, cannot burn or burn/graze. The 2011 and 2012 policies are the product of an active adaptive optimization procedure and reflect the knowledge state $\{0.375,0.125,0.25,0.25\}$, which is the discretized set of model weights in the optimal decision table (digital appendix 10) nearest to the 2011 and 2012 model weights (table 19A). Management recommendations for the 2012 and 2013 management years were derived from these policies (appendix 8a). Despite the different model weights in 2011 and 2012 (table 19A), when discretized to the nearest 0.125 , the weights for the two years reflect the same knowledge state; thus, the same decision policy was used in 2011 and 2012.

Yrs=years. SB=smooth brome. $\mathrm{CO}=$ co-dominant smooth brome and Kentucky bluegrass. KB=Kentucky bluegrass. RM=remainder. $0-30,30-45,45-60$, and $60-100$ are levels of percent native cover. 
$A$, No management restrictions

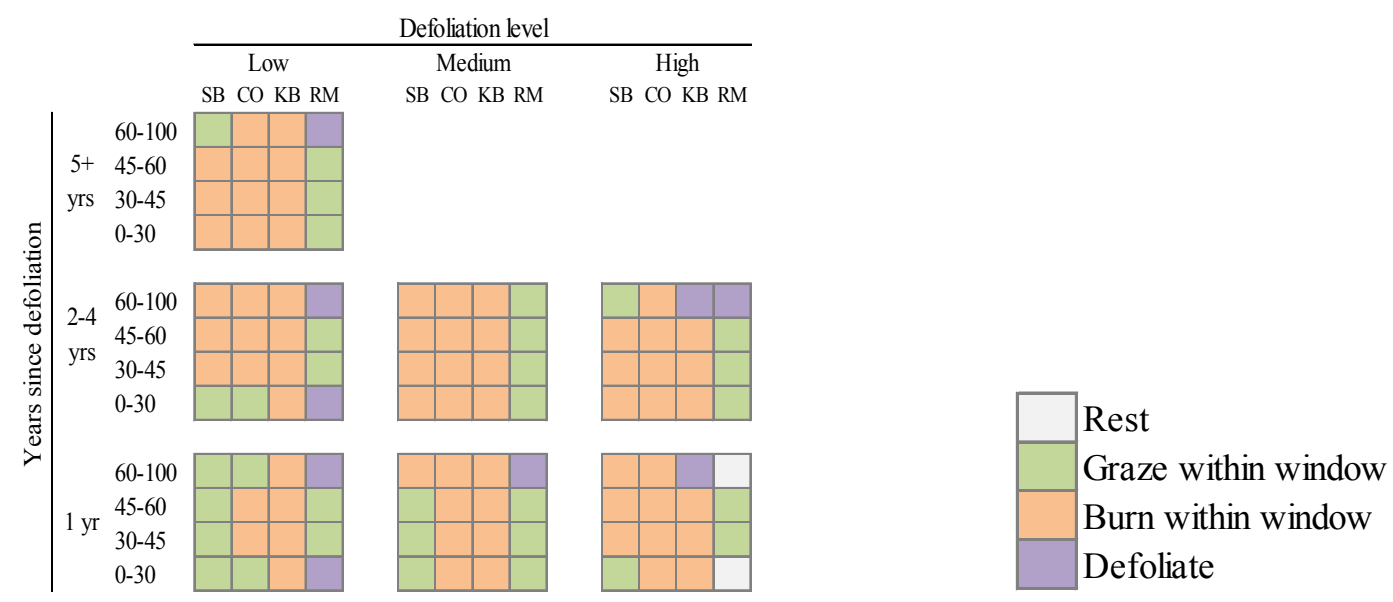

$B$, No graze

C, No burn
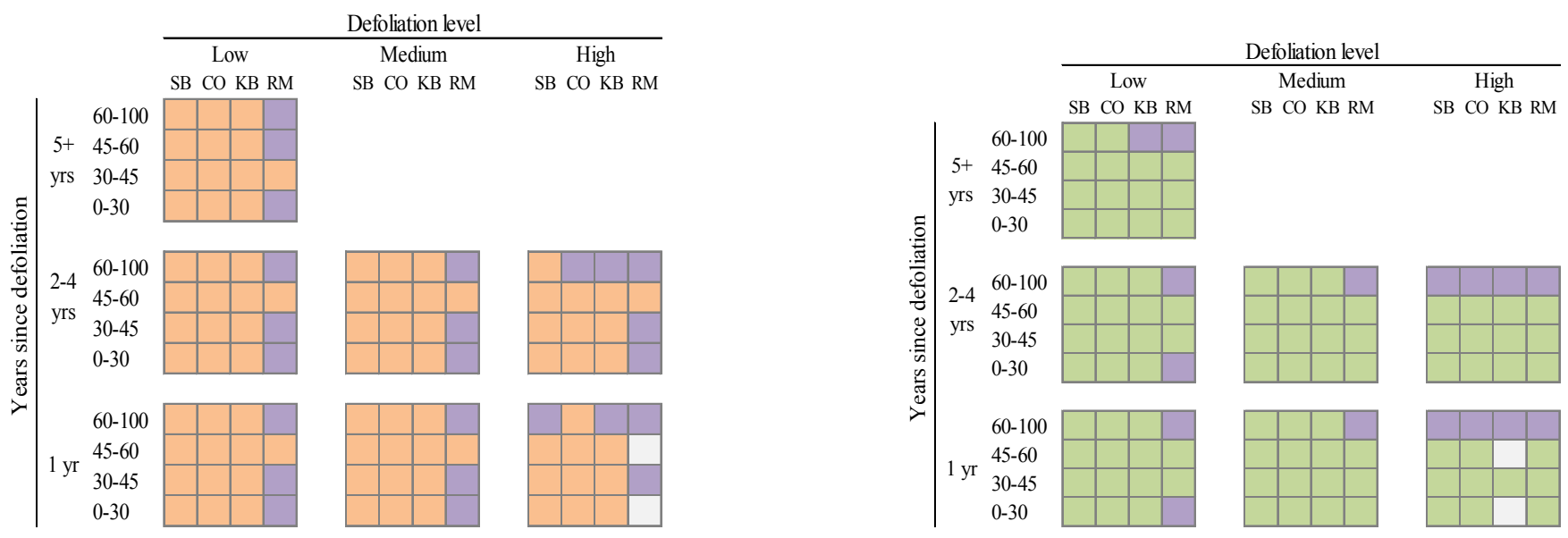

Figure 14. Tallgrass 2011 optimal decision policies for units with the following management action restrictions: $A$, no restrictions, $B$, cannot graze, and $C$, cannot burn. The 2011 policies are the product of a passive adaptive optimization procedure and reflect equal weight of 0.167 on the six alternative models in the model set. Management recommendations for the 2012 management year were derived from these policies (appendix $8 b)$. Yrs=years. $S B=$ smooth brome. $\mathrm{CO}=\mathrm{co}$-dominant smooth brome and Kentucky bluegrass. $\mathrm{KB}=$ Kentucky bluegrass. RM=remainder. 0-30, 30-45, 45-60, and 60-100 are levels of percent native cover. 
$A$, No management restrictions

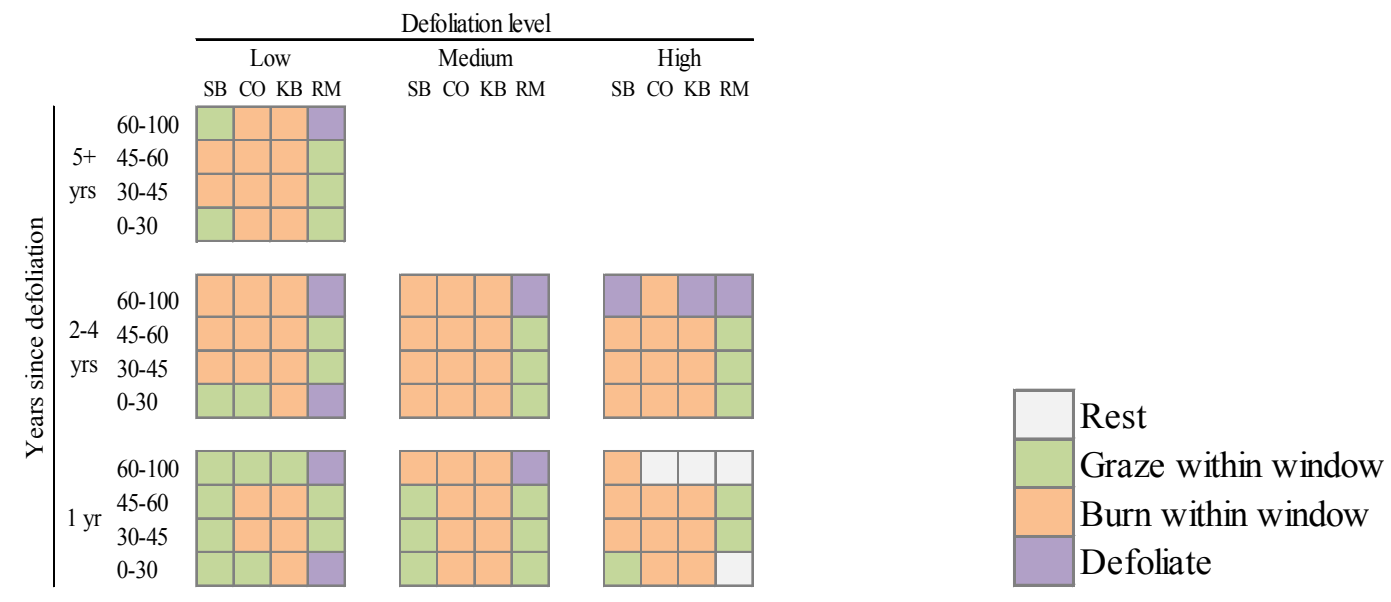

$B$, No graze

C, No burn
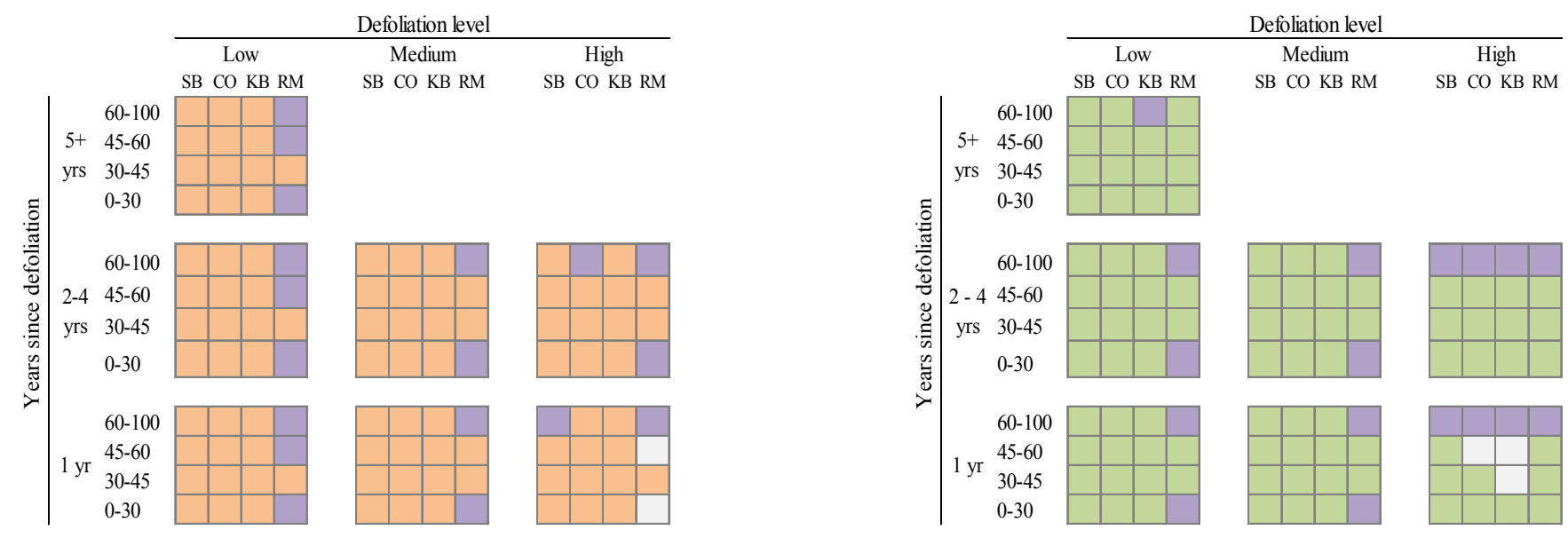

Figure 15. Tallgrass 2012 optimal decision policies for units with the following management action restrictions: $A$, no restrictions, $B$, cannot graze, and $C$, cannot burn. The 2012 policies are the product of a passive adaptive optimization procedure and reflect the knowledge state $\{0.187,0.123,0.165,0.189,0.165,0.171\}$, which is the weight on the six alternative models in the model set as determined by two updating cycles (table 19B). Management recommendations for the 2013 management year were derived from these policies (appendix 8b). Yrs=years. SB=smooth brome. $\mathrm{CO}=$ co-dominant smooth brome and Kentucky bluegrass. KB=Kentucky bluegrass. RM=remainder. $0-30,30-45,45-60$, and $60-100$ are levels of percent native cover. 


\subsection{Institutional (Double-Loop) Learning}

In addition to the technical learning that is obtained through the iterative sequence of decision making, monitoring, and assessment, adaptive management also may entail 'institutional' or 'doubleloop learning'. Such learning involves periodic reconsideration or adjustment of one or more set-up elements in the adaptive management framework (Williams and others, 2009). Although still in its infancy, NPAM has already benefitted informally from institutional learning acquired following the 2010 management cycle. The Science Team recognized a flaw in the initial set of hypotheses and models, necessitating development of a revised set of models and hypotheses along with changes to the management alternatives and inclusion of additional state variables to better reflect past management history. It was this first iteration of institutional learning that led to substantial differences in how mixed-grass and tallgrass vegetation dynamics are now considered. Recognition of the importance of double-loop learning partly motivated the formation of an advisory team.

\subsection{NPAM Advisory Team}

The NPAM system is a culmination of years of critical thinking and effort by refuge staffs, their colleagues, and their partners, and is based on substantial institutional knowledge. A great deal of thought and effort went into making the NPAM system as automated and self-sustaining as possible. Despite these considerations, the NPAM community recognized early on that the system would require periodic maintenance and re-evaluation to ensure that its original purpose was being addressed and that learning was, in fact, taking place. In developing the system, the NPAM Science Team was aware of many untested assumptions and numerous parameters that had to be estimated with minimal or no data. Although the system went through three decision cycles and three rounds of incremental improvement prior to being turned over to the FWS for full implementation, the Science Team believed that unanticipated complications were likely to arise and that capability for addressing them should be built into future work plans. The need for oversight by some form of technical committee became obvious, leading to formation of an advisory team. The following major duties of the Advisory Team were identified as follows:

(1) Periodically evaluate progress (that is, learning) under NPAM to ensure that the project does not waver from its vision of achieving improved understanding of process-oriented management of native prairies through the coordinated efforts of multiple refuges;

(2) Monitor the need for 'double-loop learning' and, should the need arise, be the driving force in determining how that need is addressed;

(3) Serve as a technical resource that the project coordinator can turn to for advice on dealing with nonroutine issues that arise;

(4) Work with the project coordinator to procure resources to solve issues deemed critical to the continued success of NPAM and identify internal or external parties equipped to address the issues;

(5) Review and respond to requests from internal (e.g., protocol modifications) and external (e.g., ancillary studies on NPAM units) parties that have the potential of affecting the integrity of NPAM or its conservation objectives;

(6) Promote NPAM to various audiences through a variety of means.

It was agreed that the project coordinator would chair the Advisory Team with assistance from a USGS representative. Membership of the Advisory Team would include users of NPAM as well as former Science Team members and developers. Initial membership of the Advisory Team would 
consist of the full Science Team plus additional members identified by the project coordinator in consultation with the Science Team. The need for developing a charter also was discussed. To date, the Advisory Team has functioned informally to address various needs, including resolving issues with the cooperator database, developing proposals for a study aimed at improving techniques for monitoring smooth brome phenology, and vetting partnership proposals from outside researchers.

\subsection{Future Improvements/Modifications}

Our project team is considering a number of modifications to be made to the NPAM program or framework elements, both in the near-term (within 2 years) and over the longer-term (longer than 2 years).

\subsubsection{Near-Term}

The near-term changes include the following:

(1) Develop a method to identify the start and end dates of the fall window in the tallgrass regionExisting phenological cues to identify the timing of the fall window are considered unsatisfactory (see section 4.3.2.2 Management Alternatives | Final Prototype | Tallgrass; footnote $^{2}$ ). Members of the Advisory Team will be pursuing further research to determine more appropriate and reliable phenological cues that can be accurately observed by cooperators to identify the timing of the fall window. Other methods besides phenological cues also may be considered (e.g., temperature, growing degree days, or simply calendar dates). It is possible that the Advisory Team will determine that a fall window does not reliably exist, in which case, actions that are implemented in the fall would be classified as 'outside the window' or 'defoliate'. Until the Advisory Team makes decisions about (1) the existence of the fall window and (2) a method for identifying the start and end dates of the fall window, tallgrass actions applied in the fall (that is, October 1 through December 31) will be considered unclassifiable and will be excluded from the annual model weight updating process.

(2) Replacing the expert judgment-based model set with a set based on empirically estimated transition probabilities - Expert judgment was used because we lacked field data to parameterize the models. With further cycles of actions and monitoring, sufficient data should soon be available to estimate the transition probabilities through empirical means, likely by formulating a set of generalized linear models that represent the competing biological hypotheses.

(3) Recomputing the optimal adaptive decision policies with replacement software for ASDP-The ASDP software has not been supported since 2002, requires an outdated $\mathrm{C}++$ compiler, and runs only on the Windows XP platform. Newer software that is available and is supported by a larger user community may provide computational efficiencies that would allow adaptive dynamic optimization of models with finer levels of discretization under reasonable time frames.

(4) Compute the active adaptive optimal decision policy for tallgrass prairie units - To date, we have used a passive adaptive optimization approach because computing the active adaptive policy with the ASDP software was impractical (see section 4.8 Optimization).

(5) Coding the model weight updating algorithm in an executable module-To compute the probability of transitions between native cover states conditional on observed cover types, we used an implementation of the Gibbs sampler in SAS. This requires that the analysis be completed outside of the Access database in an implementation of SAS available to the NPAM coordinator. This process disrupts the flow of work and requires the coordinator to acquire SAS or to seek help in running the program. A more streamlined solution is to write the updating 
algorithm in a compiled language, such as $\mathrm{C} / \mathrm{C}++$, which can be compiled as a stand-alone executable program and called directly from Access.

(6) Performing Bayesian updating on a unit-to-unit basis rather than median-of-units basisBecause current (2013) models are based on expert judgment rather than empirical analysis, we opted to use a model weight updating approach that relied on the averaging of individual unit likelihoods instead of a sequential updating approach (that is, directly substituting units for time). The averaging approach was less sensitive to large, influential likelihood outcomes, which could be an artifact of a poor model set. We expect that replacement of the current model set with one that is empirically estimated should make sequential unit-to-unit updating feasible.

(7) Complete two ancillary databases - management unit characteristics and annual phenological monitoring - and host them on the NPAM SharePoint site-The first ancillary database would store attributes of NPAM management units such as location, size, physiography, and management history. As new units are added to NPAM, the NPAM database coordinator would create new records in this database. The second ancillary database would be for input and storage of phenological observations of cool-season invasive species (smooth brome and Kentucky bluegrass) and native vegetation, which are collected at tallgrass phenology reference sites. Different observations are collected in the fall and spring and would be entered by the cooperators who collected the data. Both ancillary databases will be linked to the main NPAM Access database and will provide information necessary for the annual processing steps. The management unit ancillary database will provide information on the defoliation history of new units. The phenology ancillary database will provide information on the timing of the fall and spring tallgrass windows (that is, the dates that the windows begin and end) per location of each tallgrass management unit.

\subsubsection{Longer-Term}

Long-term modifications include the following:

(1) Account for treatment actions that are implemented during or after monitoring-In each decision cycle of adaptive management, monitoring is performed after implementation of the action. This sequence of events allows knowledge about the effects of the action to be incorporated in the updating of model weights, and it allows selection of the next action to be based on an accurate assessment of current conditions. Under NPAM, monitoring may start in June, and it may extend as late as early to mid-August. Fixing the management year at the September 1 anniversary date allows a brief window of time for all data to be entered and model weight updating to be completed. However, the logistical challenges in scheduling grazing and burning activities on refuges often result in treatment actions that partially or completely overlap the monitoring period, transpire in late August after monitoring, or extend beyond September 1 into the next management year. In all of these cases, monitoring does not measure the outcome of the implemented action, and it provides an inaccurate reading of the condition of the site for the subsequent action. The ability to manage these scenarios through model-based or analytical means is limited. The postponement of action until after monitoring could be treated as a partially controllable process, in which postponement results in a realized rest action during the inter-monitoring period, whereas the intended active treatment is carried out during the next period. With the collection of more treatment data through time, the partial controllability model can be updated with these frequencies. However, the issue of monitoring as an inaccurate indicator of condition for the next decision cycle is an example of partial observability, which is 
far more problematic for decision analysis and may not be readily solvable. After some experience with NPAM, the Advisory Team should retrospectively evaluate the timing between treatment and monitoring and consider operational options (e.g., adjustment of task deadline dates, etc.) to reduce problems caused by asynchronicity.

(2) Explore replacement of phenomenological models with process-oriented models -Existing statetransition models allow future vegetation state to be predicted from current state and a proposed management action. Those models were derived from expert opinion and lacked a mechanistic underpinning. The Science Team identified a future need to replace those first-generation models with mechanistic models that characterize underlying processes driving vegetation dynamics. Tilman (1994) presented a model of vegetation dynamics in which colonization and mortality rates of a species are mitigated by competition from co-occurring species. With this type of formulation, it becomes theoretically possible to model direct effects of a management action on colonization and mortality rates of constituent species and on the ability of species to co-occur. Replacing phenomenological models currently (2013) in use in the NPAM system with such process-oriented models would lead to improved understanding of how native and invasive species interact with one another and how each responds to a given management action. During the early stages of NPAM development, we made initial attempts to estimate colonization, mortality, and competition parameters from existing data and by expert opinion. Those efforts did not lead to a satisfactory outcome. Now that additional data on vegetation dynamics have been generated by NPAM, we believe it may be possible to achieve sufficient results.

(3) Evaluate phenological data and models, and their interaction with tallgrass treatments-A central premise of managing tallgrass native prairies in the presence of smooth brome is that phenology of smooth brome is critically important (Willson and Stubbendieck, 2000). As indicated above (section 7.2.1 Near-Term), the Advisory Team is (1) exploring opportunities to improve monitoring of smooth brome phenology and (2) questioning the existence of a fallwindow for effective management of smooth brome and Kentucky bluegrass. Answering questions concerning if and when a fall-window exists-or even to confirm existence of a spring window-may require focused research that is beyond the capability of NPAM but is clearly in NPAM's best interest. However, insights into these questions is believed possible by examining existing and future NPAM monitoring data relative to the timing of management actions.

(4) Post hoc analyses to assess utility of additional state variables, to refine estimates of partial controllability, to assess variability in how treatments are implemented, to confirm adequacy of monitoring program, and other purposes - Identifying the most critical uncertainties facing land managers was a daunting challenge for NPAM stakeholders and developers. In the end, only a few major uncertainties could be directly expressed through NPAM hypotheses and models. Many additional uncertainties were identified during meetings of NPAM cooperators and the Science Team. Although most of these potentially important uncertainties could not be built directly into NPAM, we recognized that many of them could be investigated through post hoc analyses of monitoring data. For example, insights into specific times of year at which burning is most or least effective should be possible by examining vegetation response relative to timing of treatment application. We also recognized that post hoc analyses of actual compared to recommended management actions would provide an important opportunity to refine and assess variability in estimates of partial controllability. It was further recognized that post hoc analyses of monitoring and management-action data could be helpful in (1) determining whether existing state variables were accomplishing their intended purpose and (2) identifying additional state 
variables that should be considered. Yet another benefit of post hoc analyses would be to assess the adequacy of the monitoring program itself. For example, questions related to sample size (number of transects) as it relates to precision in estimates of vegetation composition could be addressed with post hoc analyses of monitoring data.

(5) Extending NPAM to include non-FWS lands and partners-NPAM is now functioning operationally and providing optimal decision guidance for management of 120 FWS native prairie units across four states within FWS regions 3 and 6. The system is believed transportable to other publicly or privately owned prairies throughout the region. That portability has yet to be demonstrated but by replicating the NPAM decision-making process on other conservation partner lands (e.g., The Nature Conservancy, North Dakota Game and Fish) and amassing a more diverse body of data, we believe that learning can be broadened and accelerated, which would benefit both the FWS and its partners. We are currently beginning a pilot study to investigate the efficacy of porting NPAM to lands managed by other conservation partners.

\subsection{Conclusions}

We have presented the framework and details of the Native Prairie Adaptive Management system as it existed in December 2012. The system is now entering its second full year of complete operation. NPAM represents one of only a few fully implemented applications of adaptive management within the U.S. Fish and Wildlife Service. NPAM is truly unique in that it originated from the ground up as a result of the leadership and steadfastness of several refuge biologists and managers confronted with a common problem. These biologists recognized that working together across a large landscape represented perhaps the best chance for halting and reversing the invasion of native grasslands by nonnative cool-season grasses. Importantly, the NPAM system encapsulates the collective thinking and experience of tens if not hundreds of individuals who have battled this vexing problem for much of their careers.

Invasion of native prairies by introduced plants is an incremental process, whereby small changes in composition over 1-5 years go unnoticed, in part because plant populations fluctuate with environmental variation characteristic of grassland ecosystems. Restoration and maintenance of prairies will require an improved understanding of factors contributing to current ecosystem dysfunction and conversely, those necessary for restoring ecosystem health (Grant and others, 2009). The NPAM initiative is rooted in principles of adaptive management, thereby affording the opportunity for grassland managers to pursue management objectives, while acquiring information to improve future management. The invaded condition of native prairies today materialized over several decades and restoration may require as long, or longer, depending on how quickly information can be gained to better inform decision making. The NPAM system provides a foundation that integrates information collected by a diverse group of individuals across the northern Great Plains and furnishes a means to transform that information into reliable knowledge. For the first time in the history of prairie management in the northern Great Plains, detailed records of management actions are being consistently recorded as part of a large-scale coordinated effort. In addition, the response of vegetation to that management is being systematically monitored with standardized techniques.

The NPAM initiative introduced a number of technical innovations that will serve as templates for conservation efforts throughout and beyond the U.S. Fish and Wildlife Service. First, NPAM is an on-the-ground implementation of active adaptive management - possibly the first of its kind in conservation management - in which recommended management actions result from a prospective analysis of future learning (Williams, 1996). In other words, actions are chosen not only for how they 
are expected to enhance conservation outcomes in the short term but also based on information that could be returned for the attainment of long-term objectives. Second, by the use of dynamic optimization, NPAM demonstrates how decisions can be made that take into account possible future transitions of the system. Third, NPAM demonstrates how models of partial controllability are an effective means of accommodating unpredictable circumstances that cause a manager to follow a different course than was intended. Finally, the database developed for NPAM is an unparalleled system that enables the rapid integration of data from the field for the generation of 'just-in-time' management recommendations. In all, NPAM provides an example of how a science-management partnership can be forged to address large-scale conservation objectives.

\section{References Cited}

Agresti, A., 2002, Categorical data analysis (2nd ed.): Hoboken, NJ., Wiley, 734 p.

Anderson, D.R., 1975, Optimal exploitation strategies for an animal population in a Markovian environment-A theory and an example: Ecology, v. 56, p. 1281-1297.

Bailey, R.G., 1978, Ecoregions of the United States: Ogden, Utah, U.S. Forest Service, Intermountain Region, $77 \mathrm{p}$.

Bellman, R., 1957, Dynamic programming: Princeton, N.J., Princeton University Press, 366 p.

Brennan, L.A. and Kuvlesky Jr., W.P., 2005, North American grassland birds-An unfolding conservation crisis?: Journal of Wildlife Management, v. 69, p. 1-13.

Coupland, R.T., 1992, Mixed prairie, pages 151-182 in Coupland, R.T., ed., Ecosystems of the World: Natural Grasslands: New York, Elsevier, 470 p.

Grant, T.A., Flanders-Wanner, B., Shaffer, T.L., Murphy, R.K., and Knutsen, G.A., 2009, An emerging crisis across northern prairie refuges - Prevalence of invasive plants and a plan for adaptive management: Ecological Restoration, v. 27, p. 58-65.

Grant, T.A., Madden, E.M., Murphy, R.K., Smith, K.A., and Nenneman, M.P., 2004, Monitoring native prairie vegetation-The belt transect method: Ecological Restoration, v. 22, p. 106-112.

Higgins, K.F., Naugle, D.E., and Forman, K.J., 2002, A case study of changing land use practices in the Northern Great Plains, USA - An uncertain future for waterbird conservation: Waterbirds, v. 25 (Spec Pub 2), p. 42-50.

Johnson, F.A., Moore, C.T., Kendall, W.L., Dubovsky, J.A., Caithamer, D.F., Kelley, Jr., J.R., and Williams, B.K., 1997, Uncertainty and the management of mallard harvests: Journal of Wildlife Management, v. 61, p. 202-216.

Keeney, R.L., 1992, Value-focused thinking: Cambridge, Mass., Harvard University Press, 416 p.

Link, W.A., and Barker, R.J., 2010, Bayesian inference: London, Academic Press, 400 p.

Lubow, B.C., 1995, SDP-Generalized software for solving stochastic dynamic optimization problems: Wildlife Society Bulletin, v. 23, p. 738-742.

Lubow, B.C., 1997, Adaptive stochastic dynamic programming (ASDP) — Supplement to SDP user's guide, version 2.0., Fort Collins, Colo., Colorado Cooperative Fish and Wildlife Research Unit, Colorado State University, $31 \mathrm{p}$.

Lunn, D.J., Thomas, A., Best, N., and Spiegelhalter, D., 2000, WinBUGS-A Bayesian modelling framework - Concepts, structure, and extensibility: Statistics and Computing, v. 10, p. 325-337.

McCarthy, M.A., and Possingham, H.P., 2007, Active adaptive management for conservation: Conservation Biology, v. 21, p. 956-963. 
Moore, C.T. and Conroy, M.J., 2006, Optimal regeneration planning for old-growth forestAddressing scientific uncertainty in endangered species recovery through adaptive management: Forest Science, v. 52, p. 155-172.

Murphy, R.K. and Grant, T.A., 2005, Land management history and floristics in mixed-grass prairie, North Dakota, USA: Natural Areas Journal, v. 25, p. 351-358.

Nichols, J.D., Johnson, F.A., and Williams, B.K., 1995, Managing North American waterfowl in the face of uncertainty: Annual Review of Ecology and Systematics, v. 26, p. 177-199.

Otfinowski, R., Kenkel, N.C., and Catling, P.M., 2007, The biology of Canadian weeds. 134. Bromus inermis Leyess: Canadian Journal of Plant Science, v. 87, p. 183-198.

Puterman, M.L., 1994, Markov decision processes: New York, Wiley, 649 p.

Risser, P.G., Birney, E.C., Blocker, H.D., May, S.W., Parton, W.J., and Wiens, J.A., 1981, The true prairie ecosystem: Stroudsburg, Pa., Hutchinson Ross Publishing Company, 557 p.

Runge, M.C., 2011, An introduction to adaptive management for threatened and endangered species: Journal of Fish and Wildlife Management, v. 2, p. 220-233.

Samson, F.B. and Knopf, F.L., 1994, Prairie conservation in North America: Bioscience, v. 44, p. 418421.

Samson, F.B., Knopf, F.L., and Ostlie, W.R., 1998, Grasslands, pages 437-472 in Mac, M.J., Opler, P.A., Puckett Haecker, C.E., and Doran, P.D., eds., Status and trends of the nation's biological resources, Vol. 2: U.S. Department of the Interior, U.S. Geological Survey.

Samson, F., Knopf, F.L., and Ostlie, W.R., 2004, Great Plains ecosystems-Past, present, and future: Wildlife Society Bulletin, v. 32, p. 6-15.

Stubbendieck, J., 1988, Historical development of native vegetation on the Great Plains, pages 21-28 in Mitchell, J.E., ed., Impacts of the conservation reserve program in the great plains: U.S. Forest Service General Technical Report RM-158.

Tilman, D., 1994, Competition and biodiversity in spatially structured habitats: Ecology, v. 75, p. 2-16.

United States Fish and Wildlife Service, 2006, Renovation of native prairie on National Wildlife Refuge System lands in the northern Great Plains with an emphasis on reducing competition by smooth brome in Proceedings of the brome summit, Jamestown, North Dakota, 14-15 March 2006, compiler, T.A. Grant: Northern Prairie Wildlife Research Center. (Also available at http:// www.npwrc.usgs.gov/pdf/npwrc1629.pdf.)

Walters, C.J., 1986, Adaptive management of renewable resources: New York, MacMillan, 374 p.

Williams, B.K., 1996, Adaptive optimization and the harvest of biological populations: Mathematical Biosciences, v. 136, p. 1-20.

Williams, B.K., 2001, Uncertainty, learning, and the optimal management of wildlife: Environmental and Ecological Statistics, v. 8, p. 269-288.

Williams, B.K., Nichols, J.D., and Conroy, M.J., 2002, Analysis and management of animal populations: San Diego, Ca., Academic Press, 817 p.

Williams, B.K., Szaro, R.C., and Shapiro, C.D., 2007, Adaptive management-The U.S. Department of the Interior technical guide: Washington, D.C., U.S. Department of the Interior, Adaptive Management Working Group. (Also available at http://www.doi.gov/initiatives/AdaptiveManagement/TechGuide.pdf.)

Willson, G.D., and Stubbendieck, J., 2000, A provisional model for smooth brome management in degraded tallgrass prairie: Ecological Restoration, v. 18, p. 34-38. 


\section{Appendixes}


Appendix 1a. Description of exercise designed to elicit from Fish and Wildlife Service members of the Science Team information necessary to determine the vegetation state structure and the utility function.

[The elicitation was executed in two parts: (1) a curve exercise and (2) a rating exercise. The first exercise is described here, whereas the second is described in appendix 1d. The original exercise description and the accompanying Excel file that were used for the curve elicitation exercise can be found in the digital appendix 5a and digital appendix $5 \mathrm{~b}$, respectively]

\section{Curve Exercise}

The curve exercise was designed to elicit information necessary to determine two things: (1) the vegetation state structure and (2) the slope of the utility values along the diagonal of the utility matrices table 1).

Table 1: Utility matrix for the rest management action, highlighting the diagonal.

[The purpose of the exercise is to elicit information necessary to determine the appropriate division of the native cover state space into discrete groupings, and to determine the values along the diagonal of the utility matrix. $\mathrm{NP}=$ native prairie]

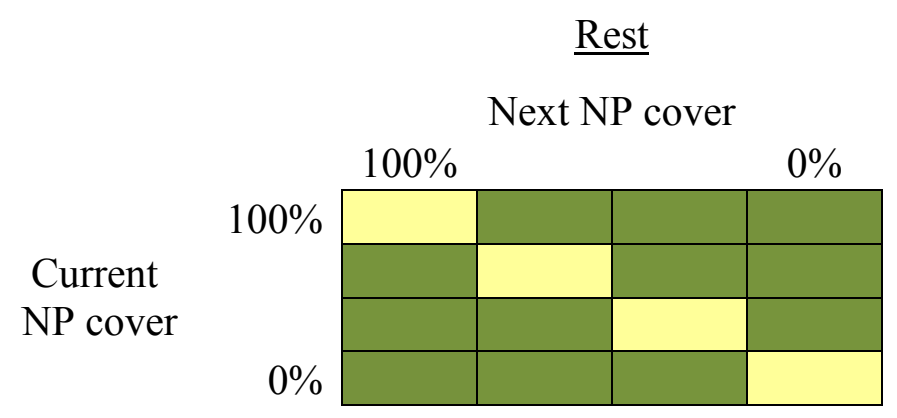

For vegetation state structure, we needed to divide up the continuous state space of percent cover of native grasses and forbs, which spans from 0 percent to 100 percent, into a manageable state space of four discrete groupings of native cover. The utility function describes how managers value different amounts of native cover, as represented by the discrete vegetation states, and is based on the vegetation state structure. For this reason, it was not appropriate to simply divide the continuous state space into four equally spaced discrete groupings (that is, $0-25$ percent, $25-50$ percent, $50-75$ percent, $75-100$ percent); rather, we wanted the structure of the four discrete groupings of native cover to be determined by how managers' value the different levels of native cover.

Although the percent of native prairie cover is quantifiable, we needed to measure a manager's relative strength of preference for management units with different percent covers of native prairie. We assumed that the higher the percent cover of native prairie, the more attractive it is; thus, we gave a management unit with 100 percent native prairie cover a value of 100 . Similarly, we assumed that the lower the percent cover of native prairie, the less attractive it is; thus, we assigned a management unit with 0 percent native prairie cover a value of 0 . But we needed to find the values of the native prairie covers that fall between the most-preferred and the least-preferred percent cover. We elicited these values through the curve exercise.

We used two different approaches for the curve exercise. The first approach was an elicitation method known as bisecting. The second approach allowed the respondents more freedom in providing their values than did the bisecting method. Respondents were allowed to use either approach, but we 
only accepted one response per respondent. The responses enabled us to create a value function and estimate the values associated with any percent native prairie cover between the most and least preferred percent cover.

Bisecting Method - For the bisecting method, respondents were asked to complete three steps, in sequence.

(1) Midpoint value: Identify the percent native prairie cover that has a value to the respondent that is halfway between the least-preferred cover ( 0 percent) and the most-preferred cover (100 percent). The respondent was provided with examples, a graph so he/she could see shape of the curve his/her response created, and an explanation of what different graph shapes mean in terms of values on native cover.

(2) 1st quarter value: Identify the percent native prairie cover that the respondent values halfway between the least-preferred cover ( 0 percent) and the midpoint-preferred cover that was identified in step 1 .

(3) 3rd quarter value: Identify the percent native prairie cover that the respondent values halfway between the midpoint-preferred cover (as identified in step 1) and the most-preferred cover (100 percent).

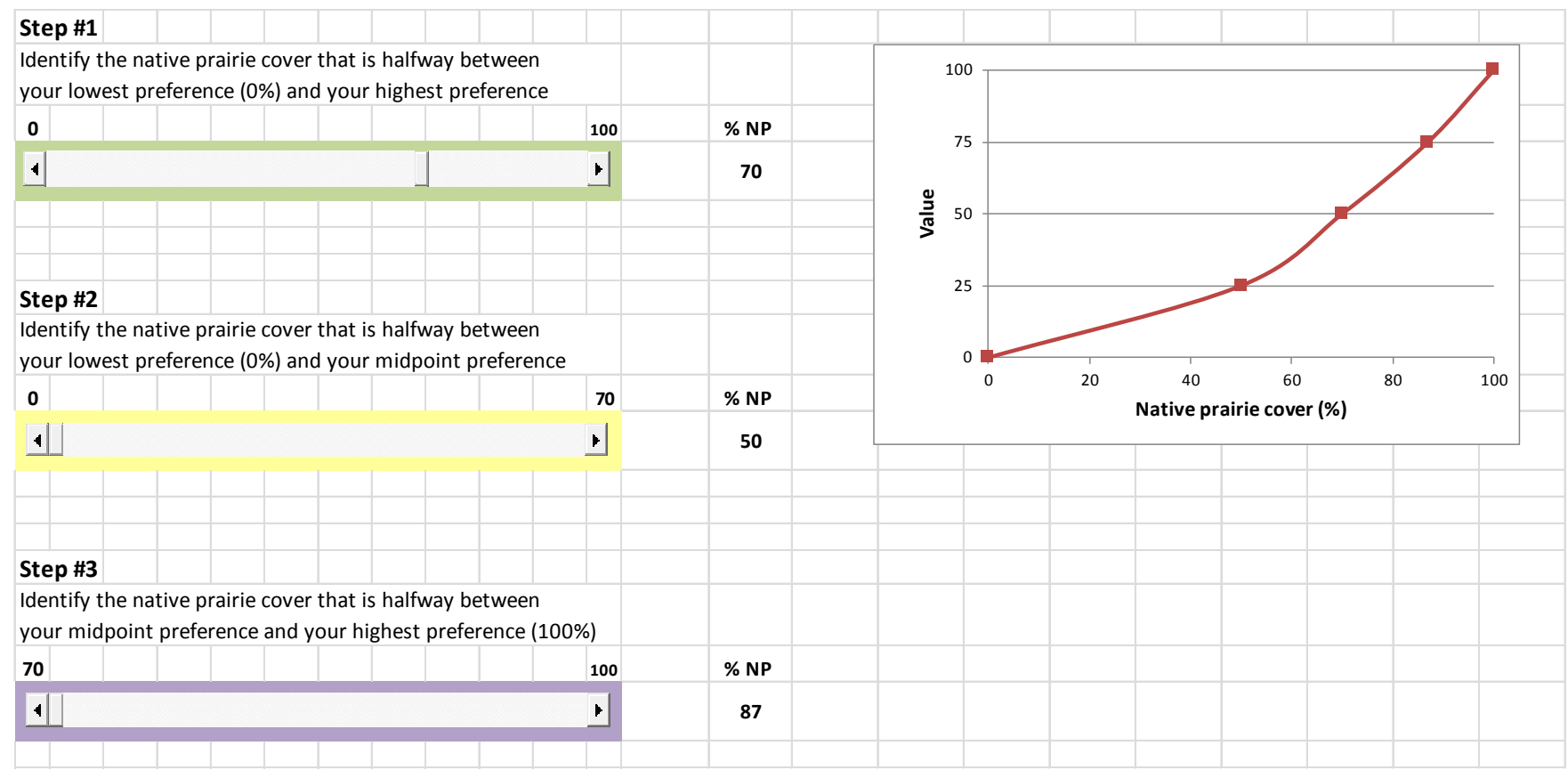

Figure 1: An example of a completed exercise using the bisecting method. NP=native prairie.

Free-form Method-If respondents were not satisfied with the curve that resulted from the responses they gave to the bisecting method, they had the option to complete a second worksheet that allowed them the freedom to directly provide values to native prairie cover without the limitation of only assigning first quarter, midpoint, and third quarter values. For this exercise, the worksheet had two columns. The first column contained percent native prairie cover that ranged from 0 percent to 100 percent and was discretized by 10 percentage points. The second column was for the respondent to fill in their values associated with each percent native cover in the first column, where we already filled in a 
value of 0 for 0 percent native prairie cover (the least-preferred cover) and a value of 100 for 100 perent native prairie cover (the most-preferred cover). The remaining cells in the second column were blank. The respondent simply provided his/her values between 0 and 100 to the native prairie cover amounts in such a way that the resulting curve matched how he/she values native prairie at these different cover amounts.

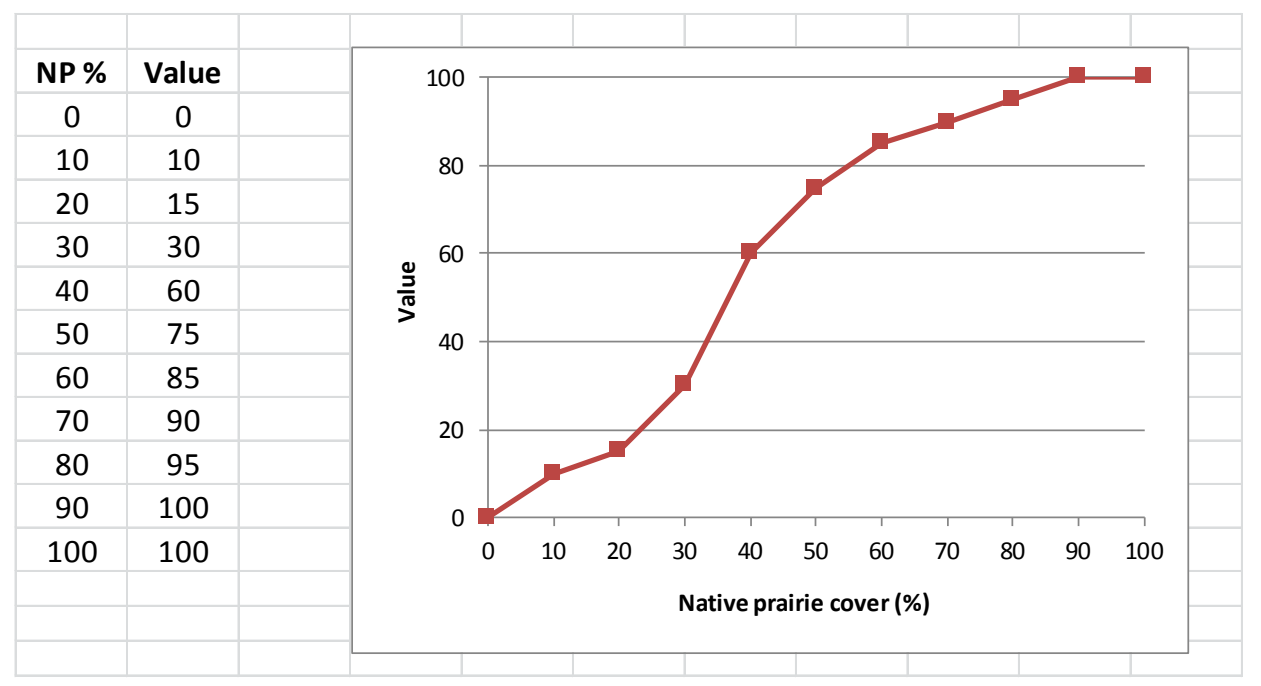

Figure 2: An example of a completed exercise using the free-form method. NP=native prairie. 
Appendix 1b. Responses of the Fish and Wildlife Service members of the Science Team for the curve exercise designed to elicit information necessary to determine the vegetation state structure and part of the utility function. [The raw responses for the curve exercise are shown here; the names of the respondents were removed and they are referred by letter]

\section{Curve Exercise}

Below is a table of values that respondents provided for the curve exercise. Respondent names are withheld and are labeled by the letters A-E. The subscript for each respondent indicates the method that the respondent used to provide a response for the exercise: $f f=$ free-form method and $b=$ bisecting method. A dash (-) in a specific column and row indicates that a response was not provided by the respondent for the native prairie cover percentage.

\begin{tabular}{c|ccccc} 
& \multicolumn{5}{c}{ Respondent values } \\
\cline { 2 - 6 } $\begin{array}{c}\text { Native } \\
\text { prairie } \\
\text { cover }\end{array}$ & $\mathrm{A}_{f f}$ & $\mathrm{~B}_{f f}$ & $\mathrm{C}_{f f}$ & $\mathrm{D}_{\mathrm{ff}}$ & $\mathrm{E}_{b}$ \\
\hline $0 \%$ & 0 & 0 & 0 & 0 & 0 \\
$10 \%$ & 1 & 3 & 5 & 10 & - \\
$20 \%$ & 20 & 8 & 10 & 15 & - \\
$30 \%$ & - & 20 & 20 & 30 & - \\
$40 \%$ & 40 & 40 & 50 & 60 & - \\
$50 \%$ & 70 & 60 & 60 & 75 & 25 \\
$60 \%$ & - & 75 & 75 & 85 & - \\
$70 \%$ & - & 90 & 95 & 90 & 50 \\
$80 \%$ & - & 95 & 98 & 95 & - \\
$90 \%$ & - & 97 & 99 & 100 & - \\
$100 \%$ & 100 & 100 & 100 & 100 & 100 \\
\cline { 3 - 6 } & & & & &
\end{tabular}


Appendix 1c. Description of the method used to determine the vegetation state structure based on the elicited responses to the curve exercise.

[The method is outlined below in numerated steps]

$\underline{\text { Step } 1}$

For each response we received, we filled in missing values by way of interpolation (table 1) and graphed the responses (fig. 1).

Table 1: Respondent values for the curve exercise.

[Values provided by the respondents are in bold, whereas interpolated values are in non-bold, italicized font. Respondents are labeled by the letters A-E. The subscript for each respondent indicates the method that the respondent used to provide a response for the exercise: $f f=$ free-form method and $b=$ bisecting method]

\begin{tabular}{c|ccccc} 
& \multicolumn{5}{c}{ Respondent values } \\
\cline { 2 - 6 } $\begin{array}{c}\text { Native } \\
\text { prairie } \\
\text { cover }\end{array}$ & $\mathrm{A}_{f f}$ & $\mathrm{~B}_{f f}$ & $\mathrm{C}_{f f}$ & $\mathrm{D}_{f f}$ & $\mathrm{E}_{b}$ \\
\hline $0 \%$ & $\mathbf{0}$ & $\mathbf{0}$ & $\mathbf{0}$ & $\mathbf{0}$ & $\mathbf{0}$ \\
$10 \%$ & $\mathbf{1}$ & $\mathbf{3}$ & $\mathbf{5}$ & $\mathbf{1 0}$ & 5 \\
$20 \%$ & $\mathbf{2 0}$ & $\mathbf{8}$ & $\mathbf{1 0}$ & $\mathbf{1 5}$ & 10 \\
$30 \%$ & 30 & $\mathbf{2 0}$ & $\mathbf{2 0}$ & $\mathbf{3 0}$ & 15 \\
$40 \%$ & $\mathbf{4 0}$ & $\mathbf{4 0}$ & $\mathbf{5 0}$ & $\mathbf{6 0}$ & 20 \\
$50 \%$ & $\mathbf{7 0}$ & $\mathbf{6 0}$ & $\mathbf{6 0}$ & $\mathbf{7 5}$ & $\mathbf{2 5}$ \\
$60 \%$ & 78.3 & $\mathbf{7 5}$ & $\mathbf{7 5}$ & $\mathbf{8 5}$ & 37.5 \\
$70 \%$ & 86.7 & $\mathbf{9 0}$ & $\mathbf{9 5}$ & $\mathbf{9 0}$ & $\mathbf{5 0}$ \\
$80 \%$ & $\mathbf{9 5}$ & $\mathbf{9 5}$ & $\mathbf{9 8}$ & $\mathbf{9 5}$ & 65.4 \\
$90 \%$ & 97.5 & $\mathbf{9 7}$ & $\mathbf{9 9}$ & $\mathbf{1 0 0}$ & 80.8 \\
$100 \%$ & $\mathbf{1 0 0}$ & $\mathbf{1 0 0}$ & $\mathbf{1 0 0}$ & $\mathbf{1 0 0}$ & $\mathbf{1 0 0}$ \\
\cline { 2 - 6 } & & & & &
\end{tabular}




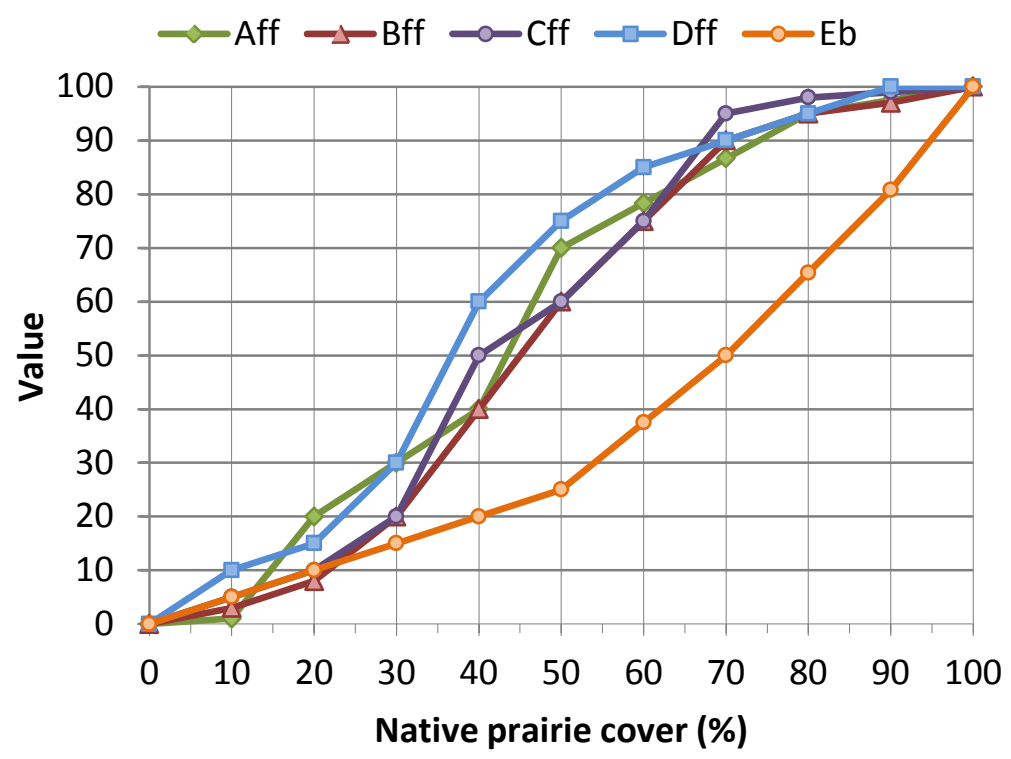

Figure 1: Values assigned to percent native prairie cover for each of the five respondents (A-E). Curves include interpolated values (table 1). $f f=$ free-form method and $b=$ bisecting method.

Four of the five responses are similar and make an $s$-shaped curve (fig. 1; respondents A-D), demonstrating that these respondents value a gain in $x$ percent native prairie less when at lower and higher amounts of total native prairie cover and more when at moderate amounts of total native prairie cover. One of the five responses is a concave-shaped curve (fig. 1; respondent E), suggesting that this respondent values a gain in $x$ percent native prairie less when at lower amounts of total native prairie cover and more when at moderate and high amounts of total native prairie cover.

\section{$\underline{\text { Step } 2}$}

We calculated the median of the five responses to arrive at a single curve (table 2; fig. 2); using the median of the five responses, as opposed to the mean, minimized effects of outlying values. 
Table 2: Median of the five responses.

[Interpolated responses were used for native prairie cover where a value was not directly provided for the discretized level of percent cover]

\begin{tabular}{c|c}
$\begin{array}{c}\text { Native } \\
\text { prairie } \\
\text { cover }\end{array}$ & $\begin{array}{c}\text { Median } \\
\text { response } \\
\text { value }\end{array}$ \\
\hline $0 \%$ & 0 \\
$10 \%$ & 5 \\
$20 \%$ & 10 \\
$30 \%$ & 20 \\
$40 \%$ & 40 \\
$50 \%$ & 60 \\
$60 \%$ & 75 \\
$70 \%$ & 90 \\
$80 \%$ & 95 \\
$90 \%$ & 97.5 \\
$100 \%$ & 100
\end{tabular}

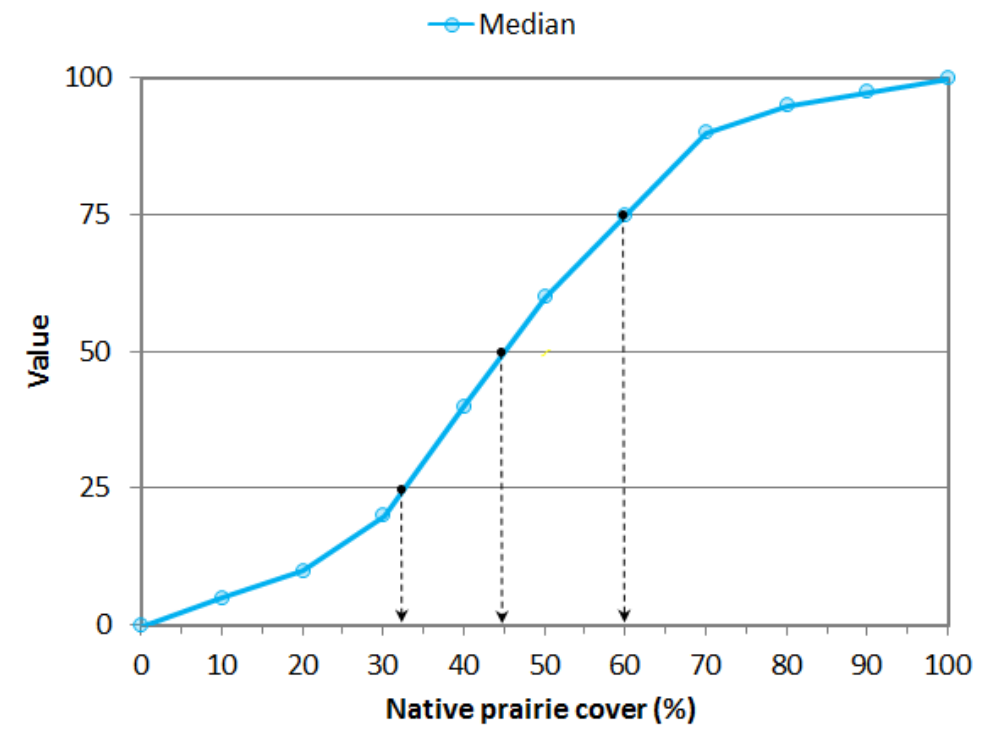

Figure 2: Median of the five responses; interpolated responses were used for native prairie cover where a value was not directly provided for the discretized level of percent cover. The dashed vertical arrows indicate the native prairie cover percentages that correspond to the quarter-value points along the $y$-axis.

We then divided the 100-point value distribution into quarters ${ }^{1}$, which provided four equal blocks of 25 points each (y-axis; fig. 2). Next, we determined the percent native prairie cover (x-axis)

\footnotetext{
${ }^{1}$ The division choice of quarters was a compromise between fine-grained representation of native cover and the need to keep the state space manageable for analysis/optimization.
} 
that corresponded to each of the 25-value break points along the y-axis (fig. 2, dashed lines with arrows; table 3).

Table 3: Percent native prairie cover corresponding to the 25-point value breaks.

\begin{tabular}{|c|c|}
\hline Value & $\begin{array}{c}\text { Native prairie } \\
\text { cover }\end{array}$ \\
\hline 25 & $32.5 \%$ \\
\hline 50 & $45 \%$ \\
\hline 75 & $60 \%$ \\
\hline
\end{tabular}

Based on the median curve, the equal division of the value space, and the corresponding native prairie cover amounts, we arrived at the following structure for dividing the native prairie state space: 0-30 percent, 30-45 percent, 45-60 percent, 60-100 percent. This division of the state space into four equally valued distributions of native prairie cover, as opposed to simply four equal distributions of native prairie cover, is desirable for defining the utility (see appendix 1d). 
Appendix 1d. Description of the rating exercise designed to elicit information necessary to determine the utility function from the Fish and Wildlife Service members of the Science Team.

[The original exercise description and the accompanying Excel file that was used for the elicitation exercise can be found in digital appendix $5 \mathrm{c}$ and digital appendix $5 \mathrm{~d}$, respectively]

\section{Rating Exercise}

After determining the state structure (appendix 1c), based on responses from the curve exercise, we asked respondents to complete the rating exercise. The rating exercise was designed to elicit information necessary to ascertain the values of the four corners of the rest matrix and the four corners of the burn/graze matrix (table 1). These eight corner values, along with the diagonal that was elicited in the curve exercise, provide the information needed to populate all of the utility matrix values.

Table 1: Utility matrices for rest and burn/ graze combination, highlighting the four corners of each matrix. [Eliciting values for these eight corners is the target of the rating exercise. $\mathrm{NP}=$ native prairie. The unit of native prairie cover is percent]
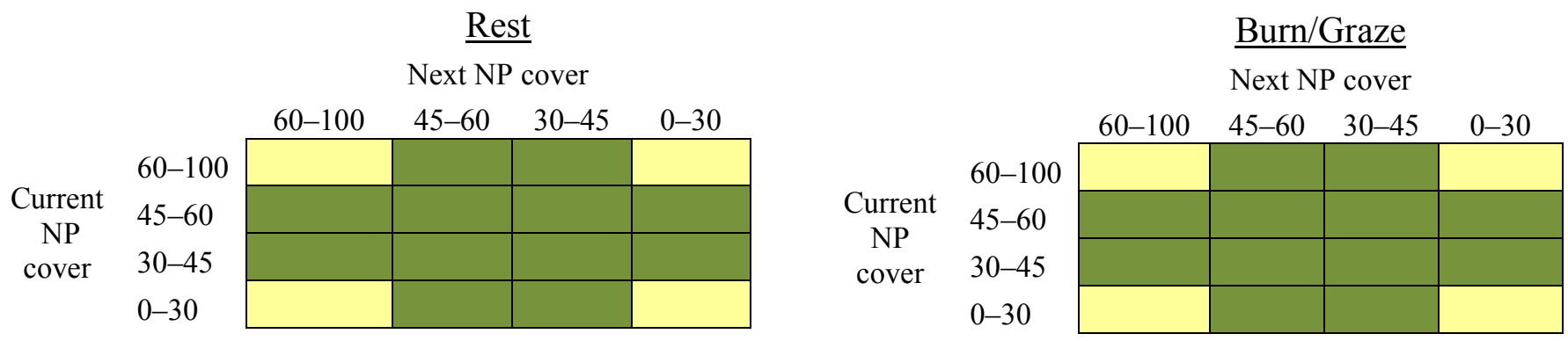

We provided an Excel workbook that contained two worksheets; the first worksheet contained the exercise, whereas the second worksheet contained an example exercise for an unrelated non-prairie problem. We held a webinar conference call with the Science Team members before sending the exercise to them, during which we reviewed the example exercise in detail and prepared them for the coming elicitation exercise.

The exercise included two steps, ranking and assigning difference values, which needed to be completed in sequence.

Ranking - The worksheet contained a table (table 2) with four outcomes that correspond to the four corners of a single utility matrix: Maintain native prairie (NP) at $60-100$ percent, Maintain NP at 0-30 percent, Increase NP from 0-30 percent to 60-100 percent, and Decrease NP from 60-100 percent to $0-30$ percent (see table 1; upper left, lower right, lower left, and upper right corners, respectively). It contained two columns, one for the rest treatment and one for the burn/graze combination treatment; these two treatments represent the two extremes in cost from least cost to greatest cost. Combining the four outcomes with the two treatments, there are eight possible outcomes; these eight outcomes represent the four corners of the rest utility matrix and the four corners of the burn/graze utility matrix (table 1). 
Table 2: Table of eight possible outcomes corresponding to the four corners of the rest and burn/graze utility matrices.

[Numbers designate the most (1) and least (8) desired of the eight possible outcomes. $\mathrm{NP}=$ native prairie]

\begin{tabular}{|c|c|c|c|}
\hline & & $\underline{\text { Tre }}$ & ents \\
\hline & & Rest & $\begin{array}{l}\text { Burn/ } \\
\text { Graze }\end{array}$ \\
\hline & Maintain NP at $60-100 \%$ & & \\
\hline & Maintain NP at $0-30 \%$ & & \\
\hline vutcomes & Increase NP from $0-30 \%$ to $60-100 \%$ & 1 & \\
\hline & Decrease NP from $60-100 \%$ to $0-30 \%$ & & 8 \\
\hline
\end{tabular}

Based on a previous discussion with the Science Team members, we had ranked the most desired outcome (1) as increasing NP from 0-30 percent to 60-100 percent without having to do any management (that is, rest), and the least desired outcome (8) as decreasing NP from 60-100 percent to 0-30 percent after applying the most expensive burn/graze treatment. We then asked each respondent to rank his/her preferences (2-7) among the remaining six outcomes according to how they lie between the most desired (rank 1) and the least desired (rank 8) outcome.

Difference Rating-After completing the ranking step described above, respondents were asked to complete the difference rating step. The worksheet contained seven two-way comparisons between sequentially ranked outcomes, which were automatically determined based on the respondent's ranking in the previous step: (1) compared ranked-outcome 1 to ranked-outcome 2; (2) compared rankedoutcome 2 to 3 ; (3) compared ranked-outcome 3 to 4 ; (4) compared ranked-outcome 4 to 5; (5) compared ranked-outcome 5 to 6; (6) compared ranked-outcome 6 to 7; and (7) compared rankedoutcome 7 to 8 . Respondents were asked to examine each of the seven two-way comparisons and identify the comparison between two sequentially ranked outcomes that was the most difficult for $\mathrm{him} /$ her to discriminate between in terms of how he/she valued them. Once identified, the respondent was instructed to assign that comparison a value of ' 1 '. Then the respondent was directed to look at the six remaining two-way comparisons and provide a value to each by comparing how different the two being compared were relative to the first two-way comparison to which he/she gave a difference value of ' 1 '. To do this, the respondent was advised to ask himself/herself how many times more different each two-way comparison was compared with the first two-way comparison that he/she set; no limit was placed on this value.

Once the respondent had assigned a difference value to each comparison, the rank, description, and scaled value (between 0 and 100) of the eight possible outcomes was shown. The scaled values were a direct result of the differences the respondent entered in the rating exercise. The scaling method was as follows: (1) the first-ranked outcome received a scaled value of 100, whereas the eighth-ranked outcome received a scaled value of 0 ; (2) 100 was divided by the sum of the difference values to provide a scaling value; (3) where $r=\operatorname{rank}(1,2, \ldots, 8), d=$ difference value, and $x=$ scaled value, starting with the second-ranked outcome, the scaled value for the second through seventh-ranked outcomes were calculated as follows:

$x_{r}=x_{r-1}-\left(d_{r-1, r} \times \frac{100}{\sum d}\right)$ 
An example of a completed elicitation worksheet, ranking and rating, is shown below for reference (fig. 1).

\begin{tabular}{|c|c|c|c|c|c|c|c|}
\hline \multicolumn{8}{|c|}{ Step \#1: Rank your preferences from the most desired (1) to the least desired (8) outcome. } \\
\hline & & & $\underline{\text { Tre }}$ & tments & & & \\
\hline & & & Rest & Burn/Graze & & & \\
\hline & \multirow{4}{*}{ Outcomes } & Maintain NP at $60-100 \%$ & 3 & 4 & & & \\
\hline & & Maintain NP at $0-30 \%$ & 5 & 6 & & & \\
\hline & & Increase NP from $0-30 \%$ to $60-100 \%$ & 1 & 2 & & & \\
\hline & & Decrease NP from $60-100 \%$ to $0-30 \%$ & 7 & 8 & & & \\
\hline & & & & & & & \\
\hline \multicolumn{8}{|c|}{ Step \#2: Two-Way Comparisons of Incrementally Ranked Outcomes } \\
\hline Comparison & \begin{tabular}{|c|c|}
$\begin{array}{c}\text { Outcome } \\
\text { Rank }\end{array}$ \\
\end{tabular} & Outcome Description & & Difference & $\begin{array}{c}\begin{array}{c}\text { Outcome } \\
\text { Rank }\end{array} \\
\end{array}$ & Outcome Description & $\begin{array}{c}\text { Scaled } \\
\text { Value }\end{array}$ \\
\hline \multirow{3}{*}{1} & 1 & Rest| Increase NP from $0-30 \%$ to $60-10$ & & \multirow{2}{*}{1} & 1 & Rest| Increase NP from $0-30 \%$ to $60-100 \%$ & 100 \\
\hline & 2 & BG |Increase NP from $0-30 \%$ to $60-10$ & & & 2 & BG | Increase NP from $0-30 \%$ to $60-100 \%$ & 98 \\
\hline & & & & & 3 & Rest | Maintain NP at $60-100 \%$ & 90 \\
\hline \multirow{3}{*}{2} & 2 & BG | Increase NP from $0-30 \%$ to $60-10$ & & \multirow{2}{*}{5} & 4 & BG |Maintain NP at $60-100 \%$ & 86 \\
\hline & 3 & Rest|Maintain NP at $60-100 \%$ & & & 5 & Rest|Maintain NP at 0-30\% & 69 \\
\hline & & & & & 6 & BG | Maintain NP at $0-30 \%$ & 60 \\
\hline \multirow{2}{*}{3} & 3 & Rest|Maintain NP at $60-100 \%$ & & \multirow{2}{*}{2} & 7 & Rest|Decrease NP from $60-100 \%$ to $0-30 \%$ & 26 \\
\hline & 4 & BG |Maintain NP at $60-100 \%$ & & & 8 & BG |Decrease NP from $60-100 \%$ to $0-30 \%$ & 0 \\
\hline \multirow{2}{*}{4} & 4 & BG |Maintain NP at $60-100 \%$ & & \multirow{2}{*}{10} & & & \\
\hline & 5 & Rest | Maintain NP at $0-30 \%$ & & & & & \\
\hline & & & & & & & \\
\hline \multirow{2}{*}{5} & 5 & Rest|Maintain NP at 0-30\% & & \multirow{2}{*}{5} & & & \\
\hline & 6 & BG | Maintain NP at $0-30 \%$ & & & & & \\
\hline \multirow{2}{*}{6} & 6 & BG | Maintain NP at $0-30 \%$ & & \multirow{2}{*}{20} & & & \\
\hline & 7 & Rest|Decrease NP from $60-100 \%$ to $0-3$ & & & & & \\
\hline \multirow{2}{*}{7} & 7 & Rest|Decrease NP from $60-100 \%$ to $0-3$ & & \multirow{2}{*}{15} & & & \\
\hline & 8 & BG | Decrease NP from $60-100 \%$ to 0 & & & & & \\
\hline
\end{tabular}

Figure 1: Example of a completed rating elicitation exercise, including the initial ranking step and the final rating

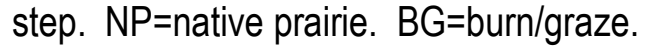


Appendix 1e. Responses of the Fish and Wild life Service members of the Science Team for the rating exercise designed to elicit information necessary to determine the utility function (appendix 1d).

[The raw responses for the exercise are shown here; the names of the respondents were removed and they are referred by letter]

$\underline{\text { Rating Exercise }}$

Below are two tables: the first for the ranks and the second for the scaled values that respondents provided for the rating exercise. Respondent names are withheld and are labeled by the letters A-G.

\begin{tabular}{|c|c|c|c|c|c|c|c|}
\hline \multirow[b]{2}{*}{ Treatment | Outcome } & \multicolumn{7}{|c|}{ Respondent Rank } \\
\hline & A & B & C & D & E & $\mathbf{F}$ & G \\
\hline Rest | Increase NP from 0-30\% to $60-100 \%$ & 1 & 1 & 1 & 1 & 1 & 1 & 1 \\
\hline BG | Increase NP from $0-30 \%$ to $60-100 \%$ & 2 & 3 & 3 & 2 & 3 & 3 & 2 \\
\hline Rest | Maintain NP at $60-100 \%$ & 3 & 2 & 2 & 3 & 2 & 2 & 3 \\
\hline BG | Maintain NP at $60-100 \%$ & 4 & 4 & 4 & 4 & 4 & 4 & 4 \\
\hline Rest | Maintain NP at 0-30\% & 5 & 5 & 5 & 5 & 5 & 5 & 5 \\
\hline BG | Maintain NP at 0-30\% & 6 & 7 & 6 & 6 & 6 & 6 & 6 \\
\hline Rest | Decrease NP from $60-100 \%$ to $0-30 \%$ & 7 & 6 & 7 & 7 & 7 & 7 & 7 \\
\hline BG | Decrease NP from $60-100 \%$ to $0-30 \%$ & 8 & 8 & 8 & 8 & 8 & 8 & 8 \\
\hline
\end{tabular}

\begin{tabular}{|c|c|c|c|c|c|c|c|}
\hline \multirow[b]{2}{*}{ Treatment | Outcome } & \multicolumn{7}{|c|}{ Respondent Scaled Values } \\
\hline & A & B & C & D & E & $\mathbf{F}$ & G \\
\hline Rest | Increase NP from 0-30\% to $60-100 \%$ & 100 & 100 & 100 & 100 & 100 & 100 & 100 \\
\hline BG | Increase NP from 0-30\% to $60-100 \%$ & 99 & 85 & 85 & 95 & 91 & 88 & 98 \\
\hline Rest | Maintain NP at $60-100 \%$ & 75 & 95 & 99 & 90 & 99 & 95 & 90 \\
\hline BG | Maintain NP at $60-100 \%$ & 55 & 80 & 84 & 85 & 90 & 78 & 86 \\
\hline Rest | Maintain NP at 0-30\% & 40 & 20 & 50 & 40 & 31 & 59 & 69 \\
\hline BG | Maintain NP at 0-30\% & 15 & 5 & 35 & 20 & 16 & 32 & 60 \\
\hline Rest | Decrease NP from $60-100 \%$ to $0-30 \%$ & 1 & 15 & 15 & 10 & 1 & 0 & 26 \\
\hline BG | Decrease NP from $60-100 \%$ to $0-30 \%$ & 0 & 0 & 0 & 0 & 0 & 0 & 0 \\
\hline
\end{tabular}


Appendix 1f. Description of the method used to determine the utility based on the elicited responses to the curve exercise and the rating exercise.

[The method is outlined below in numerated steps]

\section{Utility Matrix-Structure}

Given the native prairie state structure that was defined by the curve exercise (appendix 1c), the skeleton for a single utility matrix is shown in table 1 .

Table 1: Skeleton for a single utility matrix.

[The unit of native cover is percent. Time is referenced by $t$; where $t$ refers to current time and $t+1$ refers to the next annual time step]

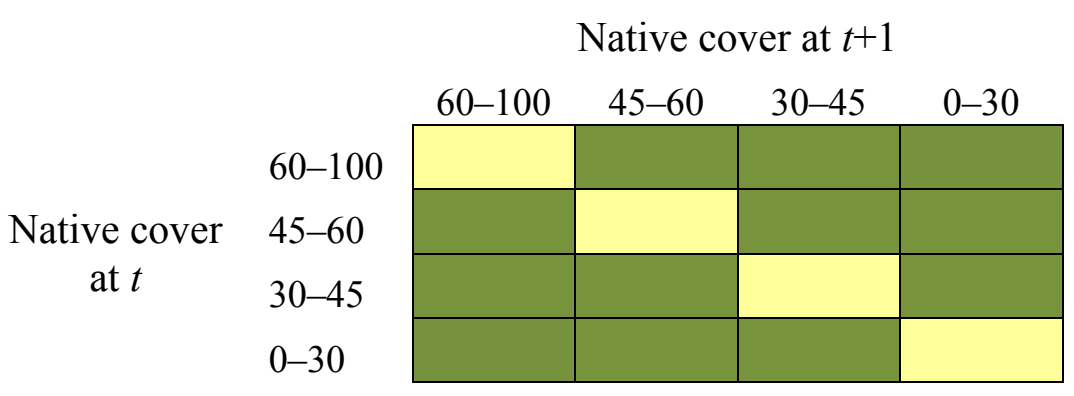

The matrix is two-dimensional, depicting the four discrete states of native cover before applying a management action at time $t$ and the native cover after applying a management action at time $t+1$. The cells along the diagonal represent maintaining a given level of native cover from time $t$ to time $t+1$. The cells below the diagonal represent moving from a lower level of native cover at time $t$ to a higher level at time $t+1$, whereas the cells above the diagonal represent moving from a higher level at time $t$ to a lower lever at time $t+1$.

\section{Utility Matrix-Slope of the Diagonal}

From the curve exercise, we calculated the value that is associated with each level of native prairie cover as the median of the values provided by the five respondents (appendix 1c). We determined the values along the diagonal of the matrix by assigning the value that corresponds to the midpoint of each discrete level of native cover (tables 2 and 3 ).

Table 2: Four levels of percent native cover, the midpoint, and the median value provided by the five respondents (appendix 1c).

\begin{tabular}{|c|c|}
\hline $\begin{array}{c}\text { Native cover } \\
\text { (midpoint) }\end{array}$ & $\begin{array}{c}\text { Median } \\
\text { value }\end{array}$ \\
\hline $\begin{array}{c}60-100 \\
(80)\end{array}$ & 95 \\
\hline $\begin{array}{c}45-60 \\
(52.5)\end{array}$ & 63.75 \\
\hline $\begin{array}{c}30-45 \\
(37.5)\end{array}$ & 35 \\
\hline $\begin{array}{c}0-30 \\
(15)\end{array}$ & 7.5 \\
\hline
\end{tabular}


Table 3: Single utility matrix with the median value of the five respondents (table 2) filled-in along the diagonal. [The unit of native cover is percent. Time is referenced by $t$; where $t$ refers to current time and $t+1$ refers to the next annual time step]

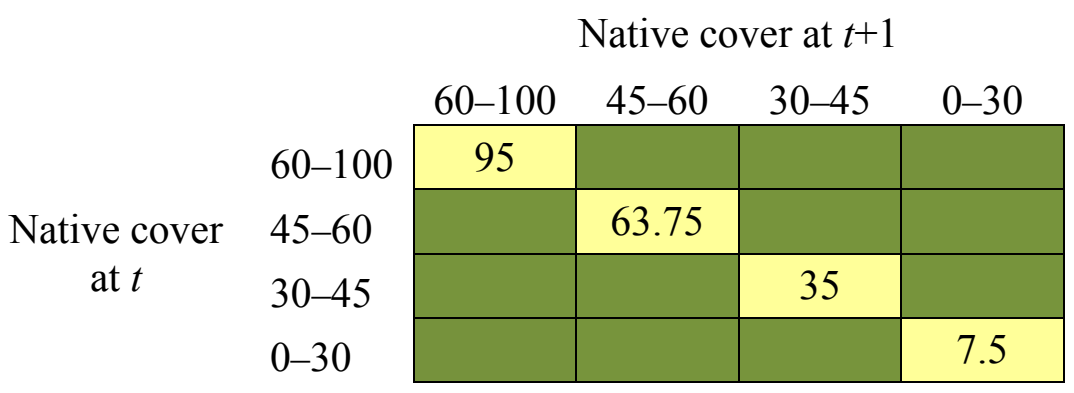

\section{Utility Matrices-The Corners}

We calculated the median of the seven responses from the rating exercise (appendixes $1 \mathrm{~d}$ and 1e) to arrive at the values of the four corners of the utility matrices for the cheapest management action, rest, and the most expensive management action, burn/graze (tables 4 and 5). Using the median of the seven responses, as opposed to the mean, minimized effects of outlying values.

Table 4: Median responses of respondents from the rating exercise (appendixes $1 \mathrm{~d}$ and $1 \mathrm{e}$ ) to populate the four corners of the rest and burn/graze utility matrices.

$[\mathrm{NP}=$ native prairie $]$

\begin{tabular}{|l|l|c|c|}
\hline Treatment & Outcome & Median & Matrix corner \\
\hline Rest & Increase NP from $0-30 \%$ to $60-100 \%$ & 100 & \multirow{2}{*}{ Lower Left } \\
Burn/Graze & Increase NP from 0-30\% to 60-100\% & 91 & \\
\hline Rest & Maintain NP at $60-100 \%$ & 95 & Upper Left \\
Burn/Graze & Maintain NP at $60-100 \%$ & 84 & \multirow{2}{*}{ Lower Right } \\
\hline Rest & Maintain NP at $0-30 \%$ & 40 & \\
Burn/Graze & Maintain NP at $0-30 \%$ & 20 & Upper Right \\
\hline Rest & Decrease NP from $60-100 \%$ to $0-30 \%$ & 10 & \\
Burn/Graze & Decrease NP from $60-100 \%$ to $0-30 \%$ & 0 & \\
\hline
\end{tabular}

Table 5: Utility matrices for rest and burn/graze with the four corners populated with the values obtained through the rating exercise (appendixes $1 \mathrm{~d}$ and $1 \mathrm{e}$ ).

$\underline{\text { Rest }}$

\begin{tabular}{|c|c|c|c|c|}
\hline & $60-100$ & $45-60$ & $30-45$ & $0-30$ \\
\hline $60-100$ & 95 & & & 10 \\
\hline $45-60$ & & & & \\
\hline $30-45$ & & & & \\
\hline $0-30$ & 100 & & & 40 \\
\hline
\end{tabular}

Burn/Graze

\begin{tabular}{l|c|c|c|c|}
\multicolumn{1}{c}{} & \multicolumn{6}{c}{$60-100$} & $45-60$ & $30-45$ & $0-30$ \\
\cline { 2 - 5 } $60-100$ & 84 & & & 0 \\
\cline { 2 - 5 } $45-60$ & & & & \\
$30-45$ & & & & \\
\cline { 2 - 5 } $0-30$ & 91 & & & 20 \\
\cline { 2 - 5 } & & & &
\end{tabular}




\section{Utility Matrices-Scaling the Diagonal}

We used the upper left and lower right corner values of the rest and burn/graze matrices (tables 4 and 5) to scale the slope of the matrix diagonal (tables 2 and 3) for the rest and burn/graze matrices. We scaled the raw diagonal values, per matrix, using the following method:

(raw diagonal value/100)*(upper left corner value-lower right corner value)+lower right corner value

Calculations for the scaled diagonal values of the rest and burn/graze matrices are shown below and the resulting matrices are shown in table 6 .

Rest

$((95 / 100) *(95-40))+40=92.25$

$((63.75 / 100) *(95-40))+40=75.0625$

$((35 / 100) *(95-40))+40=59.25$

$((7.5 / 100 *(95-40))+40=44.125$
Burn/Graze

$((95 / 100) *(84-20))+20=80.8$

$((63.75 / 100) *(84-20))+20=60.8$

$((35 / 100) *(84-20))+20=42.4$

$((7.5 / 100 *(84-20))+20=24.8$

Table 6: The final result for the scaled diagonal values of the rest and burn/graze matrix.

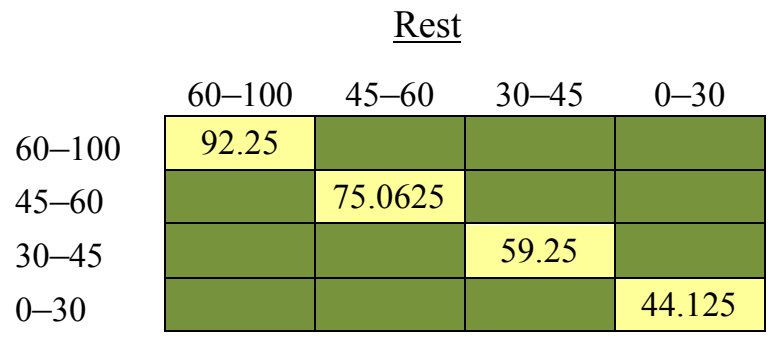

\begin{tabular}{|c|c|c|c|c|}
\hline & \multicolumn{4}{|c|}{ Burn/Graze } \\
\hline & $60-100$ & $45-60$ & $30-45$ & $0-30$ \\
\hline 60-100 & 80.8 & & & \\
\hline $45-60$ & & 60.8 & & \\
\hline $30-45$ & & & 42.4 & \\
\hline $0-30$ & & & & 24.8 \\
\hline
\end{tabular}

\section{Utility Matrices-Scaling the Corners}

Because the upper left and lower right corners are the start and end points of the diagonal, scaling the raw diagonal values by the corner values resulted in the upper left and lower right corner values to be scaled, as well. To maintain the original relationship between the values of the four corners that were elicited, we scaled the lower left and upper right elicited corner values for each matrix. We used Solver in Excel to determine the value of the lower left and upper right corners that retained the original relationship between the elicited values. We focused separately on the lower left corner and the upper right corner; in doing so, we focused on maintaining two relationships at a time (fig. 1; arrows). For the lower left corner, the relationship we strived to maintain was represented by the difference between the lower left corner and upper left corner, divided by the difference between the lower left corner and the lower right corner; that is, (LL-UL)/(LL-LR), where U=Upper and L=Lower for the first letter and $\mathrm{L}=\mathrm{Left}$ and $\mathrm{R}=\mathrm{Right}$ for the second letter. For the upper right corner, the relationship we strived to maintain was represented by the difference between the upper left corner and upper right corner, divided by the difference between the lower right corner and the upper right corner; that is, (ULUR)/(LR-UR). We used Solver to determine the value for the corner of interest in the scaled matrix that would minimize the squared difference between the relationship of the elicited values and the relationship of the scaled values. The resulting value, as well as the retained relationship value, is depicted in figure 1. Maintaining the relationship among the values within a given matrix ensured that the relationship of the values between the two matrices also was maintained. 
Rest matrix-Lower left corner

Elicited Values

\begin{tabular}{|c|l|l|l|}
\hline 95 & & & \\
\hline$\uparrow$ & & & \\
\hline$\downarrow$ & & & \\
\hline 100 & $\longleftarrow$ & & $>$ \\
\hline
\end{tabular}

$(\mathrm{LL}-\mathrm{UL}) /(\mathrm{LL}-\mathrm{LR})=0.833$

Rest matrix-Upper right corner

Elicited Values

\begin{tabular}{|c|c|c|c|}
\hline 95 & \multicolumn{1}{|c|}{10} \\
\hline & & & $\uparrow$ \\
\hline & & & $\downarrow$ \\
\hline & & & 40 \\
\hline
\end{tabular}

$(\mathrm{UL}-\mathrm{UR}) /($ LR-UR $)=2.833$

Burn/Graze matrix — Lower left corner

Elicited Values

\begin{tabular}{|l|l|l|l|}
\hline 84 & & & \\
\hline$\uparrow$ & & & \\
\hline$\downarrow$ & & & \\
\hline $91 \quad 女$ & & $\longrightarrow 2$ \\
\hline
\end{tabular}

$(\mathrm{LL}-\mathrm{UL}) /(\mathrm{LL}-\mathrm{LR})=0.986$

Burn/Graze matrix—Upper right corner

Elicted Values

\begin{tabular}{|c|c|c|c|}
\hline 84 & & \\
\hline & & & $\uparrow$ \\
\hline & & & $\downarrow$ \\
\hline & & & 20 \\
\hline
\end{tabular}

$(\mathrm{UL}-\mathrm{UR}) /(\mathrm{LR}-\mathrm{UR})=4.2$
Scaled Values

\begin{tabular}{|c|l|l|l|}
\hline 92.25 & & & \\
\hline$\uparrow$ & & & \\
\hline$\vee$ & & & \\
\hline 96.625 & & $\longrightarrow 44.125$ \\
\hline
\end{tabular}

$($ LL-UL) $/($ LL-LR $)=0.833$

Scaled Values

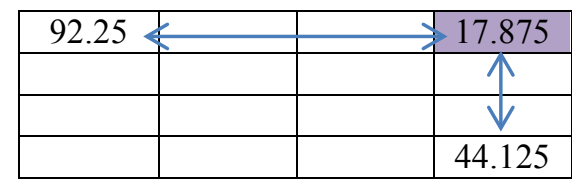

$(\mathrm{UL}-\mathrm{UR}) /(\mathrm{LR}-\mathrm{UR})=2.833$
Scaled Values

\begin{tabular}{|c|l|l|l|}
\hline 80.8 & & & \\
\hline$\uparrow$ & & & \\
\hline$\downarrow$ & & & \\
\hline 86.925 & & $\longrightarrow 24.8$ \\
\hline
\end{tabular}

$(\mathrm{LL}-\mathrm{UL}) /(\mathrm{LL}-\mathrm{LR})=0.986$

Figure 1: Elicited values and scaled values for the rest and burn/graze matrices. The arrows indicate the relationship between values, which was the focus of the newly scaled corner. The relationship value that Solver used is indicated under each matrix. UL=upper left corner of the matrix. UR=upper right corner of the matrix. $L L=l o w e r$ left corner of the matix. $L R=$ lower right corner of the matrix. The newly scaled corner is indicated by the purple-filled cell. 
Given the scaling of the diagonal per matrix, and the scaling of the corner values per matrix, the resulting values are shown in table 7.

Table 7: Scaled diagonal and corner values for the rest and burn/graze matrices.

\begin{tabular}{l|c|c|c|c|}
\multicolumn{5}{c}{$\underline{\text { Rest }}$} \\
\multicolumn{1}{c}{} & $60-100$ & $45-60$ & $30-45$ & $0-30$ \\
\cline { 2 - 5 } $60-100$ & 92.25 & & & 17.875 \\
\cline { 2 - 5 } $45-60$ & & 75.0625 & & \\
\cline { 2 - 5 } $30-45$ & & & 59.25 & \\
\cline { 2 - 5 } $0-30$ & 96.25 & & & 44.125 \\
\cline { 2 - 5 } 0 & & & &
\end{tabular}

\begin{tabular}{|c|c|c|c|c|}
\hline & \multicolumn{4}{|c|}{$\underline{\text { Burn/Graze }}$} \\
\hline & $60-100$ & $45-60$ & $30-45$ & $0-30$ \\
\hline $60-100$ & 80.8 & & & 7.3 \\
\hline $45-60$ & & 60.8 & & \\
\hline $30-45$ & & & 42.4 & \\
\hline $0-30$ & 86.925 & & & 24.8 \\
\hline
\end{tabular}

Because utility must be a value between 0 and 1, we converted all of the existing values in table 7 to a $0-1$ scale by dividing them by 100 (table 8 ).

Table 8: Scaled diagonal and corner values for the rest and burn/graze matrices, converted to a 0-1 scale.

\begin{tabular}{|c|c|c|c|c|}
\hline & \multicolumn{4}{|c|}{ Rest } \\
\hline & $60-100$ & $45-60$ & $30-45$ & $0-30$ \\
\hline $60-100$ & 0.9225 & & & 0.17875 \\
\hline $45-60$ & & 0.750625 & & \\
\hline $30-45$ & & & 0.5925 & \\
\hline $0-30$ & 0.96625 & & & 0.44125 \\
\hline
\end{tabular}

\begin{tabular}{|c|c|c|c|c|}
\hline & \multicolumn{4}{|c|}{ Burn/Graze } \\
\hline & $60-100$ & $45-60$ & $30-45$ & $0-30$ \\
\hline $60-100$ & 0.808 & & & 0.073 \\
\hline $45-60$ & & 0.608 & & \\
\hline $30-45$ & & & 0.424 & \\
\hline $0-30$ & 0.86925 & & & 0.248 \\
\hline
\end{tabular}

\section{Utility Matrices-Accounting for Treatment Cost}

Management actions incur different costs to carry them out. Because minimizing cost is part of the management objective, we must incorporate cost in the utility values. We incorporate cost by having a different matrix for each management action, where the utility is reduced by the cost of the management action. During the October 2009 NPAM annual meeting, we asked the cooperators in attendance to rank the four management actions from least expensive to most expensive. Second we assigned a value of 1 to the least expensive and a value of 10 to the most expensive. Then we asked cooperators to assign a value, between 1 and 10, to the management actions in between the least and most expensive. The result was: $1=$ rest; $5=$ graze; $8=$ burn; and $10=$ burn/graze; we refer to these values as the 'perceived relative cost'. To account for cost, in the rating exercise (appendix 1d) we elicited the four corner values of the matrices for the two management actions at either extreme: rest and burn/graze. Given the values for the rest and burn/graze matrices (table 8), along with the perceived relative cost of the four management actions, we were able to calculate a cost matrix for the four management actions (table 9). 
Table 9: Relative cost matrix for the four management actions.

[The values along the top represent the perceived relative cost of the management actions (as elicited from cooperators), where rest is the least expensive (1), burn/graze is the most expensive (10), and graze (5) and burn (8) fall in between the two extremes. The values on the left are the levels of native prairie percent cover. The values within the matrix are the cost factors for each management action, scaled between 0 and 1 . These cost factors are used in step 7 to determine the diagonal and corner utility values of the graze matrix and the burn matrix. The far left and right columns are gray to indicate that these serve as the low and high anchors, respectively, for the cost factors]

\begin{tabular}{|c|c|c|c|c|c|}
\hline & \multicolumn{4}{|c|}{ Perceived relative cost } \\
\hline & & Rest & Graze & Burn & Burn/Graze \\
\hline \multirow{5}{*}{ 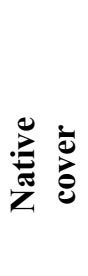 } & \multirow{3}{*}{$\begin{array}{l}60-100 \\
45-60\end{array}$} & 1 & 5 & 8 & 10 \\
\hline & & 1 & 0.944836 & 0.903463 & 0.875881 \\
\hline & & 1 & 0.915552 & 0.852216 & 0.809992 \\
\hline & \multirow{2}{*}{$\begin{array}{l}30-45 \\
0-30\end{array}$} & 1 & 0.873605 & 0.778809 & 0.715612 \\
\hline & & 1 & 0.805351 & 0.659364 & 0.56204 \\
\hline
\end{tabular}

The cost matrix (table 9) contains the cost factors for each management action, scaled between 0 and 1. The cost factor is used as a multiplicative; thus, a cost factor of 1 means there is no reduction in utility value, whereas a cost factor less than one indicates a reduction in utility where the lower the factor, the higher the reduction. Since rest incurs no financial cost, we set the cost factor of rest to 1. Using the utility values along the diagonals of the rest and burn/graze matrices (table 8), we determined the cost of burn/graze relative to rest by dividing the utility value of burn/graze by the utility value of rest. We did this for each native cover value along the diagonal and arrived at the cost factor for burn/graze. With the scaled cost factor for rest and burn/graze, along with the elicited perceived relative cost of the four management actions, we determined the scaled cost factor of the graze action and the burn action per level of native cover. The cost factor for carrying out action $i$ at native cover level $j$ was calculated as follows:

$\frac{\left(C F_{R j}-C F_{B G j}\right)}{\left(P C_{B G}-P C_{R}\right)} \times\left(P C_{B G}-P C_{i}\right)+C F_{B G j}$

where

$\mathrm{CF}=$ cost factor

$\mathrm{PC}=$ perceived relative cost

$\mathrm{R}=$ rest

$\mathrm{BG}=$ burn/graze

$i=$ graze or burn

$j=$ native cover level $0-30,30-45,45-60$, or $60-100$

Completing the cost matrix revealed an interesting quality about cooperator values. Cooperators place less importance on the cost of a treatment when the treatment is being carried out to maintain an already high level of native cover. Cost is considered more relevant (that is, there is a greater reduction in utility value) when the same treatment is carried out to maintain or increase the native cover of a unit that currently has a lower level of native cover. 


\section{Utility Matrices-Determining Diagonal and Corner Values for the Graze Matrix and Burn Matrix}

Diagonal Values - The diagonal values $(a, b, c$, and $d$; table 10) of the graze matrix and the burn matrix were calculated by multiplying the diagonal values of the rest matrix (table 8 ) by the appropriate cost factor (table 9). The appropriate cost factor is determined by the management action ( $i$; graze or burn) and the level of native cover at time $t(j ; 0-30,30-45,45-60$, or 60-100).

Corner Values - The off-diagonal corner values ( $x$ and $y$; table 10) of the graze and burn matrices were calculated using the off-diagonal corner values of the rest and burn/graze matrices (table 8) along with the appropriate cost factors (table 9) as follows:

$$
\begin{aligned}
& x_{G}=x_{B G}+\left(x_{R}-x_{B G}\right) \times\left(C F_{G, 0-30}-C F_{B G, 0-30}\right) /\left(C F_{R, 0-30}-C F_{B G, 0-30}\right) \\
& y_{G}=y_{B G}+\left(y_{R}-y_{B G}\right) \times\left(C F_{G, 60-100}-C F_{B G, 60-100}\right) /\left(C F_{R, 60-100}-C F_{B G, 60-100}\right) \\
& x_{B}=x_{B G}+\left(x_{R}-x_{B G}\right) \times\left(C F_{B, 0-30}-C F_{B G, 0-30}\right) /\left(C F_{R, 0-30}-C F_{B G, 0-30}\right) \\
& y_{B}=y_{B G}+\left(y_{R}-y_{B G}\right) \times\left(C F_{B, 60-100}-C F_{B G, 60-100}\right) /\left(C F_{R, 60-100}-C F_{B G, 60-100}\right)
\end{aligned}
$$

where

$$
\begin{aligned}
& C F=\text { cost factor } \\
& R=\text { rest } \\
& G=\text { graze } \\
& B=\text { burn } \\
& B G=\text { burn/graze }
\end{aligned}
$$

\begin{tabular}{|c|c|c|c|c|c|}
\hline & \multicolumn{4}{|c|}{ Native cover at time $t+1$} \\
\hline & & $60-100$ & $45-60$ & $30-45$ & $0-30$ \\
\hline \multirow{4}{*}{$\begin{array}{c}\text { Native } \\
\text { cover at } \\
\text { time } t\end{array}$} & $60-100$ & $a$ & & & $y$ \\
\hline & $45-60$ & & $b$ & & \\
\hline & $30-45$ & & & $c$ & \\
\hline & $0-30$ & $x$ & & & $d$ \\
\hline
\end{tabular}

Table 10: Diagonal $(a, b, c, d)$ and off-diagonal $(x, y)$ cells of the matrix.

[The utility values in these cells for the graze matrix and the burn matrix were calculated based on the diagonal values of the rest matrix (table 8), the corner values of the rest and burn/graze matrices (table 8), and the appropriate cost factors (table 9), as described in step 7]

\begin{tabular}{|c|c|c|c|c|}
\hline & $60-100$ & $45-60$ & $30-45$ & $0-30$ \\
\hline $60-100$ & 0.833444 & & & 0.096500 \\
\hline $45-60$ & & 0.639694 & & \\
\hline $30-45$ & & & 0.461444 & \\
\hline $0-30$ & 0.890806 & & & 0.290944 \\
\hline
\end{tabular}

The resulting diagonal and corner values for the graze matrix and the burn matrix, calculated by the above methods, are shown in table 11.

Table 11: Calculated values of the utility values along the diagonal and corners for the graze matrix and the burn matrix.

\section{$\underline{\text { Graze }}$}

\begin{tabular}{l|c|c|c|c|}
\multicolumn{1}{c}{} & $60-100$ & $45-60$ & $30-45$ & $0-30$ \\
\cline { 2 - 5 } $60-100$ & 0.871611 & & & 0.131750 \\
\cline { 2 - 5 } $45-60$ & & 0.687236 & & \\
$30-45$ & & & 0.517611 & \\
\cline { 2 - 5 } $0-30$ & 0.923139 & & & 0.355361 \\
\hline
\end{tabular}

Burn 


\section{Utility Matrices-Filling in all the Cells}

In this section, we describe how we determined the values for the other cells of the matrices given the known diagonal and corner values of all four matrices (rest and burn/graze, table 8; graze and burn, table 11). The cells below the diagonal represent moving from a lower level of native cover at time $t$ to a higher level at time $t+1$ (that is, improving between time steps), whereas the cells above the diagonal represent moving from a higher level of native cover at time $t$ to a lower level at time $t+1$ (that is, degrading between time steps). There is a relationship between the known cell values ( $a, d, x$, and $y$; table 10) that determines what we call the improve factor $(\alpha)$ and the degrade factor $(\beta)$. Below we outline the steps we used to determine the improve and degrade factors and to calculate the values of the remaining cells based on the two factors $(\alpha, \beta)$ and the known cell values $(a, b, c, d)$.

Table 12: Known values $(a, b, c, d, x, y)$, improve factor $(\alpha)$, degrade factor $(\beta)$, and the equations used to determine the cell values of a given matrix.

\begin{tabular}{cl|c|c|c|c|} 
& \multicolumn{5}{c}{ Native cover at time $t+1$} \\
\cline { 3 - 6 } & $60-100$ & $60-100$ & $45-60$ & $30-45$ & $0-30$ \\
\cline { 3 - 6 } Native & $45-60$ \\
cover at & $a$ & $b-\beta(a-b)$ & $c-\beta(a-c)$ & $y$ \\
time $t$ & $30-45$ & $a+\alpha(a-b)$ & $b$ & $c-\beta(b-c)$ & $d-\beta(b-d)$ \\
\cline { 3 - 6 } & $0-30$ & $a+\alpha(a-c)$ & $b+\alpha(b-c)$ & $c$ & $d-\beta(c-d)$ \\
\cline { 3 - 6 } & & $x$ & $b+\alpha(b-d)$ & $c+\alpha(c-d)$ & $d$ \\
& & & &
\end{tabular}

Known values

- Variables $a, b, c$, and $d$ are known diagonal values, whereas $x$ and $y$ are known corner values of the matrix for each management action; these values are shown for the rest and burn/graze matrices in table 8 and for the graze and burn matrices in table 11.

Unknown values

- $\alpha$ is the improve factor.

- $\beta$ is the degrade factor.

Equations

- $x=a+\alpha(a-d)$

- $y=d-\beta(a-d)$

Calculate the unknown $\alpha$ and $\beta$ based on the known variables and equations 3 and 4 , such that:

- $\alpha=(x-a) /(a-d)$

- $\beta=-(y-d) /(a-d)$

When calculating the improve and degrade factors, we used logit values $(\log [\mathrm{z} /(1-\mathrm{z})])$ of the known cells $(x, y, a, d)$ to account for the low and high boundaries of 0 and 1 on the utility values. The improve and degrade factors were calculated separately for each matrix; that is, the improve and degrade factors are specific to the matrix of each of the four management actions (table 13). We used the improve and degrade factors, along with logit values of the known variables $(a, b, c, d)$ to calculate the values in the remaining cells of the matrices using the equations in table 12. We then backtransformed all the values using the inverse logit to get the final matrix values (table 14). 
Table 13: Improve $(\alpha)$ and degrade $(\beta)$ factors for each management action.

\begin{tabular}{l|c|c|} 
& \multicolumn{1}{l}{$\begin{array}{c}\text { Improve } \\
\text { factor }\end{array}$} & $\begin{array}{c}\text { Degrade } \\
\text { factor }\end{array}$ \\
\cline { 2 - 3 } Rest & 0.32 & 0.48 \\
\cline { 2 - 3 } Graze & 0.23 & 0.51 \\
\cline { 2 - 3 } Burn & 0.20 & 0.54 \\
\cline { 2 - 3 } Burn/Graze & 0.18 & 0.56 \\
\cline { 2 - 3 } & &
\end{tabular}

The effect of the improve factor is to increase the utility value when an improvement is made from a lower level of native prairie cover at time $t$ to a higher level of native cover at time $t+1$. This increase in utility is relative to the values along the diagonal that represent maintaining a given level of native cover between time step $t$ and $t+1$. The effect of the degrade factor is to decrease the utility value, relative to the diagonal value, when a loss of native cover occurs between time step $t$ and $t+1$. It is noteworthy that the degrade factors are larger than the improve factors, which means a higher penalty is incurred when native cover decreases than benefit is gained when native prairie increases. It is also interesting that the improve factor decreases as the cost of the management action increases, whereas the degrade factor increases as the cost of the management action increases. This relationship reveals that cooperators feel better about gaining native cover for less investment and they feel worse about losing native cover when they have invested more in management.

\section{The Complete Utility Function}

Mixed-Grass-Based on the elicitation responses for the curve and rating exercises and steps 1 through 8 outlined above, we derived the complete utility function (table 14). This utility function is specific to mixed-grass prairie units, because it covers the four alternative management actions available for mixed-grass units. In the next section we cover the utility function for the tallgrass units.

Table 14: The complete utility function for mixed-grass units including a matrix for each of the four management actions.

\begin{tabular}{l|c|c|c|c|}
\multicolumn{5}{c}{ Rest } \\
& $60-100$ & $45-60$ & $30-45$ & $0-30$ \\
\cline { 2 - 6 } $60-100$ & 0.9225 & 0.610361 & 0.348765 & 0.17875 \\
\cline { 2 - 5 } $45-60$ & 0.948903 & 0.750625 & 0.507162 & 0.294897 \\
\cline { 2 - 5 } $30-45$ & 0.959183 & 0.792052 & 0.5925 & 0.371437 \\
\cline { 2 - 5 } $0-30$ & 0.96625 & 0.822709 & 0.639173 & 0.44125 \\
\cline { 2 - 6 } & & & &
\end{tabular}

\begin{tabular}{|c|c|c|c|c|}
\hline & \multicolumn{4}{|c|}{$\underline{\text { Graze }}$} \\
\hline & $60-100$ & 45-60 & $30-45$ & $0-30$ \\
\hline & 0.871611 & 0.551729 & 0.293727 & 0.13175 \\
\hline $45-60$ & 0.897669 & 0.687236 & 0.4261 & 0.213156 \\
\hline $0-45$ & 0.91169 & 0.721133 & 0.517611 & 0.281355 \\
\hline-30 & 0.923139 & 0.750526 & 0.555225 & 0.355361 \\
\hline
\end{tabular}

Burn

\begin{tabular}{|c|c|c|c|c|}
\hline & & 15 & r & $0-30$ \\
\hline & 0.833 & 0.504102 & 0.248947 & 0.0965 \\
\hline & 0.85 & 0.639 & 0.36 & 0.157214 \\
\hline & re & 0.671 & 0. & 0.2 \\
\hline 30 & 306 & 0.702723 & 0.497339 & 0.290944 \\
\hline
\end{tabular}

Burn/Graze

\begin{tabular}{l|c|c|c|c|}
\multicolumn{1}{c}{} & $60-100$ & $45-60$ & $30-45$ & $0-30$ \\
\cline { 2 - 6 } $60-100$ & 0.808 & 0.469416 & 0.216368 & 0.073 \\
\cline { 2 - 6 } $45-60$ & 0.834286 & 0.608 & 0.326171 & 0.121309 \\
\cline { 2 - 5 } $30-45$ & 0.851972 & 0.639398 & 0.424 & 0.173516 \\
\cline { 2 - 5 } $3-30$ & 0.86925 & 0.671933 & 0.459543 & 0.248 \\
\cline { 2 - 5 } 0
\end{tabular}


Tallgrass - Tallgrass prairie units have a different set of alternative management actions and thus require a separate utility function that includes a matrix for each of the tallgrass-specific management actions: rest, defoliate, graze within window, and burn within window. The same elicitation exercises, elicitation responses, and steps 1-8 outlined above also apply to developing the utility function for tallgrass units. The tallgrass utility matrix for rest is the same as the mixed-grass utility matrix for rest. Additionally, the tallgrass utility matrix for graze within window is the same as the mixed-grass utility matrix for graze, whereas the tallgrass utility matrix for burn within window is the same as the mixed-grass utility matrix for burn. The difference for the tallgrass utility function is that we drop the burn/graze utility matrix, because burn/graze is not one of alternative management actions for tallgrass units, and we add a utility matrix for the tallgrass-specific management action defoliate. To add a matrix for the defoliate action, we return to step 6 and account for the cost of defoliate. Lacking a cooperator-elicited perceived relative cost for defoliate, compared with the other alternative management actions, we estimated the perceived relative cost for defoliate to be a ' 4 '. With this estimate, we calculated the cost matrix to include defoliate (table 15).

Table 15: Relative cost matrix for all alternative management actions of mixed-grass and tallgrass units.

[The values along the top represent the perceived relative cost of the management actions, where rest is the least expensive (1), burn/graze is the most expensive (10), and defoliate (4), graze within window (5), and burn within window (8) fall in between the two extremes. The values on the left are the levels of native prairie percent cover. The values within the matrix are the cost factors for each management action, scaled between 0 and 1 . Although burn/graze is not an alternative management action for tallgrass units, we show it here because it, along with the rest action, is used as an anchor to scale the cost factors for the other management actions: defoliate, graze within window, and burn within window. The far left and right columns are gray to indicate that these serve as the low and high anchors, respectively, for the cost factors. We treat the tallgrass actions graze within window and burn within window the same as the mixed-grass actions graze and burn, respectively]

\begin{tabular}{|c|c|c|c|c|c|c|}
\hline & & & & rceived relati & $\operatorname{cost}$ & \\
\hline & & Rest & Defoliate & $\begin{array}{c}\text { Graze within } \\
\text { window }\end{array}$ & $\begin{array}{c}\text { Burn within } \\
\text { window }\end{array}$ & Burn/Graze \\
\hline & & 1 & 4 & 5 & 8 & 10 \\
\hline & $60-100$ & 1 & 0.958627 & 0.944836 & 0.903463 & 0.875881 \\
\hline$\stackrel{\Xi}{\Xi}$ & $45-60$ & 1 & 0.936664 & 0.915552 & 0.852216 & 0.809992 \\
\hline 之े & $30-45$ & 1 & 0.905204 & 0.873605 & 0.778809 & 0.715612 \\
\hline & 0-30 & 1 & 0.854013 & 0.805351 & 0.659364 & 0.56204 \\
\hline
\end{tabular}

Given the cost factors for the management action defoliate (table 15), we proceeded through steps 7 and 8 as described above to determine the utility values in the utility matrix for the defoliate management action. The improve and degrade factors are shown in table 16. 
Table 16: Improve $(\alpha)$ and degrade $(\beta)$ factors for each alternative tallgrass management action.

[The table includes the same values as shown in table 13 for rest, graze, and burn; however, we dropped the factors for the burn/graze action and added the factors for the defoliate action]

Rest

Defoliate

Graze within window

Burn within window

\begin{tabular}{|c|c|}
\hline $\begin{array}{l}\text { Improve } \\
\text { factor }\end{array}$ & $\begin{array}{c}\text { Degrade } \\
\text { factor }\end{array}$ \\
\hline 0.32 & 0.48 \\
\hline 0.24 & 0.51 \\
\hline 0.23 & 0.51 \\
\hline 0.20 & 0.54 \\
\hline
\end{tabular}

The final utility function for tallgrass units, including a matrix for the four alternative tallgrass management actions, is shown in table 17.

Table 17: The complete utility function for tallgrass units including a matrix for each of the four alternative tallgrass management actions.

\begin{tabular}{|c|c|c|c|c|}
\hline & \multicolumn{4}{|c|}{ Rest } \\
\hline & $60-100$ & $45-60$ & $30-45$ & $0-30$ \\
\hline 100 & 0.9225 & 0.610361 & 0.348765 & 0.17875 \\
\hline $45-60$ & 0.948903 & 0.750625 & 0.507162 & 0.294897 \\
\hline $30-45$ & 0.959183 & 0.792052 & 0.5925 & 0.371437 \\
\hline-30 & 0.96625 & 0.822709 & 0.639173 & 0.44125 \\
\hline
\end{tabular}

\begin{tabular}{|c|c|c|c|c|}
\hline & \multicolumn{4}{|c|}{$\underline{\text { Defoliate }}$} \\
\hline & $60-100$ & $45-60$ & $30-45$ & $0-30$ \\
\hline & 0.884333 & 0.566865 & 0.307934 & 0.1435 \\
\hline $5-60$ & 0.910352 & 0.703 & 0.446 & 0.232621 \\
\hline 30-45 & 0.923538 & 0.737981 & 0.536333 & 0.303406 \\
\hline-30 & 0.933917 & 0.767198 & 0.575089 & 0.376833 \\
\hline
\end{tabular}

Graze within window

\begin{tabular}{|c|c|c|c|c|}
\hline & & $45-$ & 30-45 & -30 \\
\hline & 0.871611 & 0.551729 & 0.293727 & 13175 \\
\hline & 0.897 & 0.687236 & 0.4261 & 0.213156 \\
\hline-6 & $0 . S$ & 0.7 & 0.517611 & 0.281355 \\
\hline & 32. & 0.7505 & 0.5552 & 0.355 \\
\hline
\end{tabular}

\begin{tabular}{l|c|c|c|c|}
\multicolumn{1}{c}{} & \multicolumn{6}{c}{$60-100$} & $45-60$ & $30-45$ & $0-30$ \\
\hline $60-100$ & 0.833444 & 0.504102 & 0.248947 & 0.0965 \\
\cline { 2 - 6 } $\begin{array}{l}45-60 \\
30-45\end{array}$ & 0.859692 & 0.639694 & 0.366652 & 0.157214 \\
\cline { 2 - 5 } $0-30$ & 0.876003 & 0.671817 & 0.461444 & 0.216357 \\
\cline { 2 - 5 } & 0.890806 & 0.702723 & 0.497339 & 0.290944 \\
\hline
\end{tabular}


Appendix 2. Outline of the provisional Native Prairie Adaptive Management (NPAM) decision framework; the first complete, functional, but provisionary, version of the NPAM decision framework used to complete the first adaptive management cycle during management year 2010|2011.

\section{Setup Phase}

Problem: Loss of native prairie to cool-season invasive grasses smooth brome and Kentucky bluegrass

Area of focus: Native sod on Fish and Wildlife Service (FWS) owned lands across the Prairie Pothole Region in FWS regions 3 and 6

Objective: Increase the cover of native prairie (grasses, forbs) at the least cost

Alternatives: Rest, hay, graze, burn, burn/graze.

- Same set of alternative actions for mixed-grass and tallgrass units

State structure: Vegetation state of each unit was described by the native cover level and the dominant invasive. The five levels of percent native cover $(0-20,20-50,50-80,80-95,95-100)$ were based on USGS Science Team members' best estimate to equally divide the value that FWS cooperators placed on varying levels of native cover. Within levels of native prairie (NP) cover, we recognized the dominant invasive as smooth brome (SB), Kentucky bluegrass (KB), co-dominant smooth brome and Kentucky bluegrass (CO), or some other undesirable species (RM). The rules of dominance were the same as the final framework. The result was 16 discrete states that a unit could be in at any point in time.

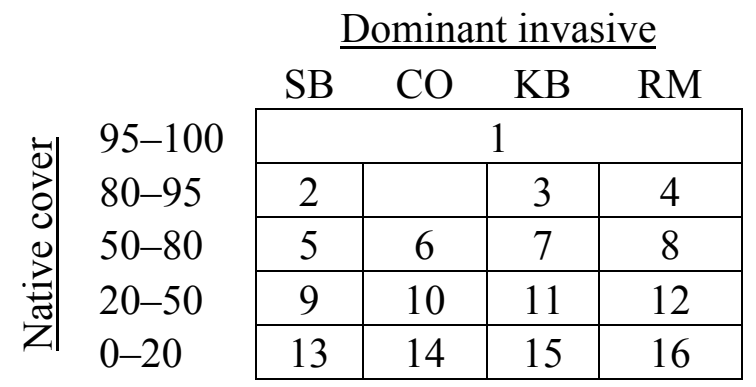

Models: State transition probability model

- Model structure: $16 \times 16$ matrix per alternative management action; one complete model consisted of five $16 \times 16$ matrices.

- Predict: Probability of being in vegetation state $1-16$ at time $t+1$ given the vegetation state at time $t$ and the management action carried out between $t$ and $t+1$ monitoring.

- Main uncertainties:

- Elicitation exercise with FWS Science Team to identify four main uncertainties:

(1) Effect of haying on NP, SB, KB

(2) Effectiveness of burning against $\mathrm{SB}$

(3) Effectiveness of grazing against KB

(4) Existence of threshold, below which management is ineffective 
- Conceptual Models: Represent structural uncertainty through four alternative models

○ Model 1: Conventional wisdom

- Base model, based on the following general agreements:

- Natural disturbance mimics (burn or graze) are good for NP.

- Haying is no better than rest.

- Grazing is better than burning against SB.

- Burning is better than grazing against KB.

$\circ$ Model 2: Haying more effective than Rest

- Hypothesized that haying is more effective than rest

- Comparison to Model 1 isolated the uncertainty about the effectiveness of haying.

- Model 3: Invader-specific treatment effectiveness

- Hypothesized that:

- Burning no better than rest on SB.

- Grazing no better than rest on KB.

- Comparison to Model 1 isolated the uncertainties about effectiveness of burning against SB and grazing against $\mathrm{KB}$.

○ Model 4: Threshold

- Hypothesized that no form of management is better than rest when NP is less than 20 percent

- Comparison to Model 1 isolated the uncertainty about the existence of a threshold.

\section{- Parameterization:}

○ Empirical model

- Meta-analysis of long-term field studies, available refuge data, and expert opinion about the effect of rest on NP, SB, and KB.

- We estimated average transition probabilities and placed vague prior probability distributions on the transitions matrices to provide inference support for the many parameters where we had sparse data.

- Estimates were completed separately for each grass type: mixed-grass and tallgrass.

- Alternative models were parameterized by tweaking parts of the empirical model to reflect the conceptual model.

\section{Utility:}

- Parameterization:

- USGS members of the Science Team made guesstimates about how the NPAM cooperators valued maintaining the different levels of native cover (values along the diagonal), gaining native cover (improve factor of 0.5), and losing native cover (degrade factor of 0.9) between time steps.

- The relative cost of the five alternative management actions (rest, hay, graze, burn, and burn/graze) was derived from an elicitation of the NPAM cooperators present at the second annual cooperator meeting. The result was $1,3,5,8$, and 10 , respectively.

- We used the same utility for mixed-grass and tallgrass. 


\begin{tabular}{|c|c|c|c|c|c|c|}
\hline \multirow[t]{2}{*}{ REST } & & \multicolumn{4}{|c|}{ Native cover at time $t+1$} & \multirow[b]{2}{*}{$0-20$} \\
\hline & & $95-100$ & $80-95$ & $50-80$ & $20-50$ & \\
\hline \multirow{5}{*}{ 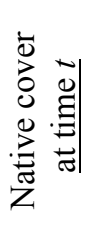 } & $95-100$ & 1.00 & 0.40 & 0.03 & 0.01 & $\mathbf{0 . 0 0}$ \\
\hline & $80-95$ & 1.00 & 0.80 & 0.13 & 0.01 & 0.00 \\
\hline & $50-80$ & 1.00 & 0.90 & 0.35 & 0.02 & 0.01 \\
\hline & $20-50$ & 1.00 & 0.95 & 0.58 & 0.10 & 0.01 \\
\hline & $0-20$ & 1.00 & 0.98 & 0.74 & 0.23 & 0.01 \\
\hline
\end{tabular}

HAY

Native cover at time $t+1$

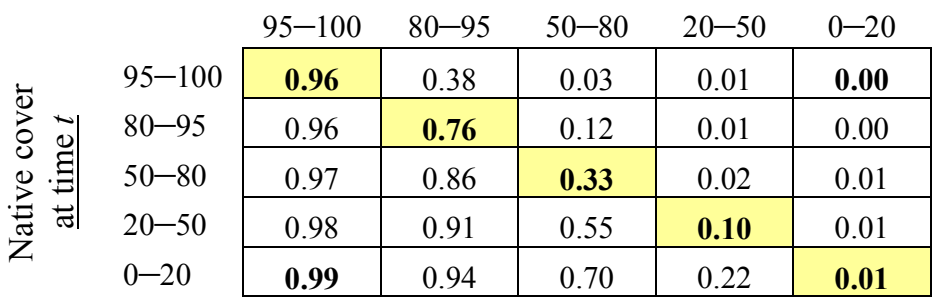

\section{GRAZE}

Native cover at time $t+1$

\begin{tabular}{|c|c|c|c|c|c|c|}
\hline \multirow{6}{*}{ 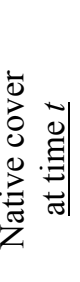 } & & $95-100$ & $80-95$ & $50-80$ & $20-50$ & $0-20$ \\
\hline & $95-100$ & 0.91 & 0.36 & 0.03 & 0.01 & 0.00 \\
\hline & $80-95$ & 0.93 & 0.73 & 0.11 & 0.01 & 0.00 \\
\hline & $50-80$ & 0.94 & 0.82 & 0.32 & 0.02 & 0.00 \\
\hline & $20-50$ & 0.96 & 0.87 & 0.52 & 0.09 & 0.01 \\
\hline & $0-20$ & 0.98 & 0.91 & 0.67 & 0.21 & 0.01 \\
\hline
\end{tabular}

BURN Native cover at time $t+1$

\begin{tabular}{|c|c|c|c|c|c|c|}
\hline \multirow{5}{*}{ 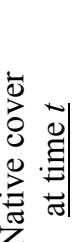 } & & $95-100$ & $80-95$ & $50-80$ & $20-50$ & $0-20$ \\
\hline & \multirow{2}{*}{$\begin{array}{l}95-100 \\
80-95\end{array}$} & 0.84 & 0.33 & 0.02 & 0.00 & 0.00 \\
\hline & & 0.87 & 0.68 & 0.11 & 0.01 & 0.00 \\
\hline & $50-80$ & 0.90 & 0.76 & 0.30 & 0.02 & 0.00 \\
\hline & $20-50$ & 0.93 & 0.82 & 0.49 & 0.08 & 0.01 \\
\hline & $0-20$ & 0.96 & 0.86 & 0.62 & 0.19 & 0.01 \\
\hline
\end{tabular}

BURN/GRAZE Native cover at time $t+1$

\begin{tabular}{|c|c|c|c|c|c|c|}
\hline \multirow{5}{*}{ 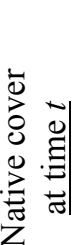 } & & $95-100$ & $80-95$ & $50-80$ & $20-50$ & $0-20$ \\
\hline & \multirow{2}{*}{$\begin{array}{l}95-100 \\
80-95\end{array}$} & 0.80 & 0.32 & 0.02 & 0.00 & 0.00 \\
\hline & & 0.84 & 0.64 & 0.10 & 0.01 & 0.00 \\
\hline & $50-80$ & 0.88 & 0.72 & 0.28 & 0.02 & 0.00 \\
\hline & $20-50$ & 0.91 & 0.78 & 0.46 & 0.08 & 0.01 \\
\hline & $0-20$ & 0.95 & 0.83 & 0.59 & 0.18 & 0.01 \\
\hline
\end{tabular}




\section{Partial Controllability}

- Parameterization:

- Elicitation exercise with the FWS members of the Science Team. Three team members responded for mixed-grass and a different three responded for tallgrass.

\section{Mixed-Grass}

\begin{tabular}{|c|c|c|c|c|c|}
\hline \multirow[b]{2}{*}{$\begin{array}{l}\text { Recommended } \\
\text { action }\end{array}$} & \multicolumn{5}{|c|}{ Implemented action } \\
\hline & Rest & Hay & Graze & Burn & Burn/Graze \\
\hline Rest & 0.73 & 0.02 & 0.15 & 0.10 & 0.01 \\
\hline Hay & 0.46 & 0.20 & 0.17 & 0.13 & 0.04 \\
\hline Graze & 0.28 & 0.00 & 0.57 & 0.12 & 0.03 \\
\hline Burn & 0.30 & 0.02 & 0.13 & 0.40 & 0.15 \\
\hline Burn/Graze & 0.43 & 0.00 & 0.18 & 0.17 & 0.22 \\
\hline
\end{tabular}

\section{Tallgrass}

\begin{tabular}{l|c|c|c|c|c|}
\multirow{2}{*}{$\begin{array}{c}\text { Recommended } \\
\text { action }\end{array}$} & \multicolumn{6}{c}{ Implemented action } \\
\cline { 2 - 6 } & Rest & Hay & Graze & Burn & Burn/Graze \\
\hline Rest & 0.80 & 0.02 & 0.08 & 0.08 & 0.02 \\
\cline { 2 - 6 } Hay & 0.27 & 0.50 & 0.05 & 0.17 & 0.02 \\
\cline { 2 - 6 } Graze & 0.12 & 0.10 & 0.46 & 0.27 & 0.05 \\
\cline { 2 - 6 } Burn & 0.10 & 0.08 & 0.03 & 0.75 & 0.03 \\
\cline { 2 - 6 } & 0.12 & 0.08 & 0.13 & 0.27 & 0.40 \\
\cline { 2 - 6 }
\end{tabular}

\section{Optimization:}

- Essentially the same process, using adaptive stochastic dynamic programming (ASDP), but used different input (models, utility, partial controllability) and thus obtained different output

- Completed separately for mixed-grass and tallgrass

\section{Monitoring:}

- Used the same monitoring protocol

\section{Iterative Phase}

\section{0 updating cycle:}

- We did not update model weights this cycle. We used assumed complete uncertainty among four alternative models for mixed-grass and among the four alternative models for tallgrass; thus, each model had a weight of 0.25 . 


\section{Optimal Policies}

- Below are the mixed and tall optimal policies used to provide the 2011 management recommendations.

Mixed-Grass

$\underline{\text { Dominant invader }}$

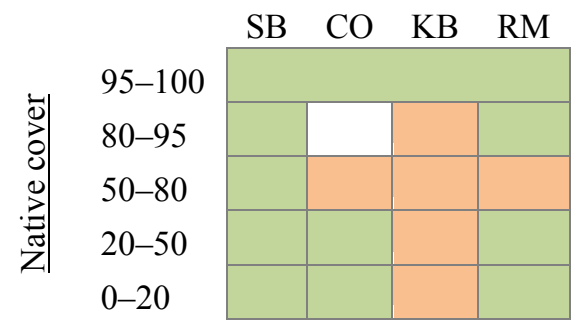

Tallgrass

$\underline{\text { Dominant invader }}$

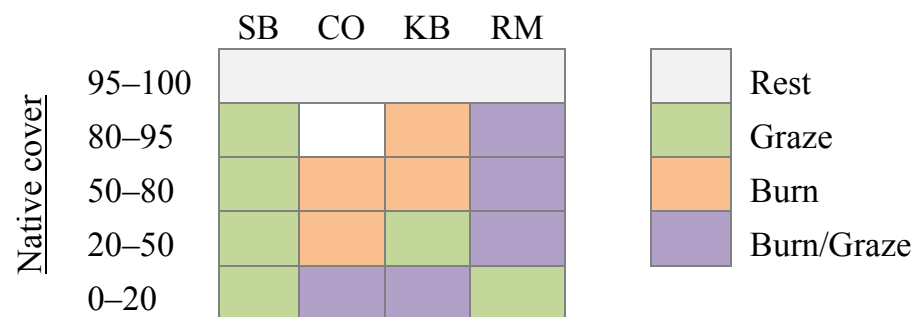

\section{Changes to provisional 2010 Framework for final 2011 Framework}

\section{Some Problems Identified}

- There was no biological argument for the effect of haying.

- Burn/Graze treatment did not appear in the recommended policy for mixed-grass units.

- Strings of the same management action could be recommended year-after-year.

- Failed to acknowledge differences between mixed-grass and tallgrass

- Failed to capture the main structural uncertainties

\section{$\underline{\text { Setup Phase-Modified Components }}$}

\section{State structure}

- Vegetation structure:

- Modified the vegetation state structure by changing the levels of native cover

- Still have 16 discrete states

- Defoliation structure:

- Added defoliation state to the state structure: includes 7 states

- Combined, there are $16 \times 7=112$ discrete states that a unit can be in at any one time.

\section{Management Alternatives}

- Different alternatives for mixed-grass and tallgrass

- Mixed-grass

- Dropped hay

$\circ$ Tallgrass

- Added the concept of the cool-season window

- Grouped hay into defoliate 


\section{Models}

- We recognized that the 2010 provisional model set failed to capture the main uncertainties; therefore, we re-elicited the cooperators to elucidate the major uncertainties.

- These uncertainties were grass type specific; therefore we formulated a different list of uncertainties per grass type, which gave rise to a different set of conceptual models per grass type.

- Models were parameterized by eliciting expert opinion from members of the FWS Science Team.

\section{Utility}

- We modified the structure of the utility matrices to reflect the change in vegetation state structure.

- We populated the values of the utility matrices through elicitation exercises with the FWS Science Team members.

\section{Partial Controllability}

- We populated the values of the partial controllability matrices through elicitation of all NPAM cooperators, per individual management unit.

\section{Optimization}

- We re-ran the optimization with ASDP with the new input, which resulted in new optimal decision tables.

\section{Iterative Phase}

- Based on changes to the setup phase components, we completed the iterative phase.

- We updated mixed-grass model weights with 2009|2010 and 2010|2011 data, which resulted in the 2011 decision policy and management recommendations for 2012 .

- We used equal model weights for tallgrass, and the non-adaptive optimal decision table to derive the 2011 decision policy and 2012 recommendations for tallgrass units. 
Appendix 3a. Model elicitation spreadsheet approach and rationale for mixed-grass baseline model.

\section{Process of Completing the Elicitation}

We lead a facilitated elicitation exercise to complete the model elicitation spreadsheet for the baseline mixed-grass model. A USGS Science Team member proposed a set of initial values for the spreadsheet; these initial values were based on the conceptual model and guidance that was provided by the Fish and Wildlife Service (FWS) members of the Science Team. The set of values was then posed to the chosen representative and expert from the mixed-grass part of the FWS Science Team for review and consideration. The USGS team member then walked through the spreadsheet in a methodical fashion with the FWS representative, discussed the existing values, and either received confirmation on the values or instruction on how to modify the values. The completed elicitation spreadsheet is located in appendix $4 \mathrm{a} 1$.

\section{Summary of Conceptual Model 3 (Baseline Model)}

- Management action effectiveness is invader-specific as follows:

- Smooth brome (SB): Burn/Graze $=$ Graze $>$ Burn $>$ Rest

- Co-dominant (CO): Burn $/$ Graze $>$ Burn $=$ Graze $>$ Rest

- Kentucky bluegrass (KB): Burn/Graze $=$ Burn $>$ Graze $>$ Rest

- Management actions have different effectiveness depending on whether the defoliation level is low or high, as follows:

- Rest is less detrimental under a high defoliation level than under a low defoliation level.

- At a low nataive prairie (NP) cover starting state (that is, 20 percent), active management actions are more effective under a high defoliation level than under a low defoliation level.

- Management action effectiveness is not density-dependent.

○ We do not expect the effectiveness of management actions to decrease when the level of invasion increases.

- Active management actions are expected to be better than rest at all levels of invasion.

\section{Approach to Thinking about Probability}

- When distributing the 100 points for each scenario, imagine 100 different one-year time-steps.

- One can consider the same management unit and imagine how a one-year time step would look if repeated 100 different times, over 100 different years. This approach would allow one to consider, for example, different weather conditions and chance events.

- One also can consider one-year time steps in the same year for 100 different management units. This approach would allow one to consider different abiotic conditions (e.g., precipitation and soil), variability in the application of the same management action on different units, and chance events.

- Use increments no smaller than five when distributing the points.

\section{Terminology}

- bar: Refers to the black line in each column of the spreadsheet that denotes the bin containing the NP starting state (that is, 80 percent or 20 percent).

- above the bar: Refers to the bins above the black line; points in these bins indicate that NP has remained the same or increased and the dominant invasive has remained the same or decreased. 
- below the bar: Refers to the bins below the black line; points in these bins indicate that NP has decreased and the dominant invasive has increased.

\section{General Approach: Upper Section-Changes in Native Cover}

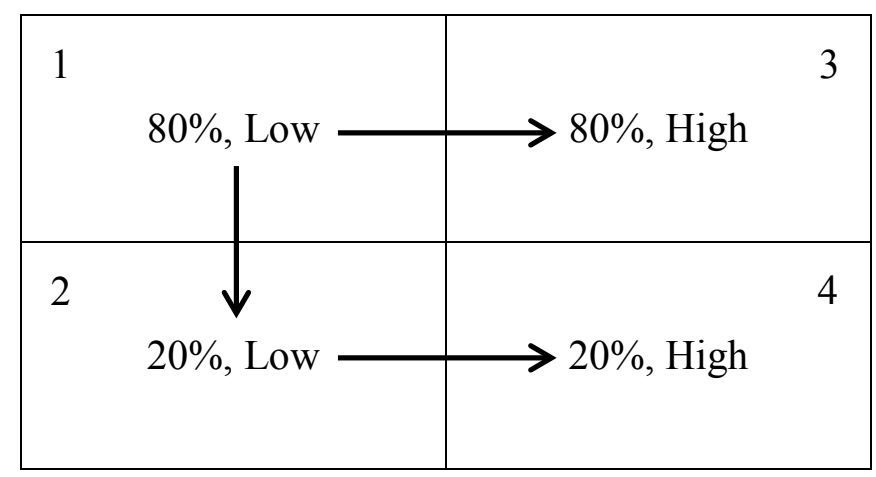

Figure 1: Simplified diagram of the first section of the elicitation spreadsheet (see table $10 \mathrm{~A}$ within the main text for the detailed spreadsheet) for changes in native cover. The diagram is divided into four quadrants, which are labeled 1-4. Quadrant 1 pertains to starting NP cover of 80 percent and low defoliation level. Quadrant 2 corresponds to starting NP cover of 20 percent and low defoliation level. Quadrant 3 represents starting NP cover of 80 percent and high defoliation level. Quadrant 4 corresponds to starting NP cover of 20 percent and high defoliation level.

Order of filling in values

(1) Set values in quadrant 1 (starting NP cover 80 percent, defoliation level low).

(2) Set values in quadrant 2 (starting NP cover 20 percent, defoliation level low) relative to values in quadrant 1.

(3) Set values in quadrant 3 (starting NP cover 80 percent, defoliation level high) relative to values in quadrant 1.

(4) Set values in quadrant 4 (starting NP cover 20 percent, defoliation level high) relative to values in quadrant 2 .

\section{Specific Approach: Upper Section-Changes in Native Cover}

\section{Quadrant 1: Starting NP Cover 80 Percent, Defoliation Level Low}

- Following the conceptual model, we created the following patterns with the probability distribution above and below the bar (indicated as above|below):
○ Rest
- SB: $20 \mid 80$
- CO: $25 \mid 75$
- KB: $30 \mid 70$
- Remainder (RM): $50 \mid 50$
- Active Management Actions
- SB
- Graze: $80 \mid 20$
- Burn: 60|40 
- Burn/Graze: $80 \mid 20$

- $\mathrm{CO}$

- Graze: $70 \mid 30$

- Burn: 70|30

- Burn/Graze: 85|15

- $\mathrm{KB}$

- Graze: $50 \mid 50$

- Burn: 80|20

- Burn/Graze: $80 \mid 20$

- $\mathrm{RM}$

- Graze: $80 \mid 20$

- Burn: 80|20

- Burn/Graze: 80|20

- Within these general patterns, the way in which the points above and below the bar are distributed among the bins is different; the differences are intentional and are described below.

- While staying true to the conceptual model, we incorporated the idea of the dubious effect of burn on SB and graze on KB.

- We did this by allotting more probability below the bar, when compared with the alternative active management actions, while still making the actions more beneficial than detrimental (in the case of burning SB, 60|40), or at least equally beneficial and detrimental (in the case of grazing KB, 50|50), and while still making the actions better than doing nothing (that is, rest).

- We incorporated the idea that $\mathrm{SB}$ is more aggressive than $\mathrm{KB}$. We incorporated this in two different ways:

- We modified the distribution of rest for the various dominant components. SB has 80 percent of the probability below the bar, whereas KB has 70 percent below the bar, and the co-dominant has 75 percent below the bar. We made RM the least aggressive, with a $50 \mid 50$ chance of decreasing or increasing with rest.

- We modified the distribution for grazing SB and burning KB. These are the choice management actions against these specific dominant invasive species. The amount of probability above the bar is equal in both cases (that is, 80 percent); however, we made burning slightly more effective against KB than grazing is against SB by pushing five of the points to a bin further above the bar in the case of burning KB.

- In the case of RM dominant, we made all active management actions 80|20 (above|below), which is the same general pattern as the management actions that are considered to be most effective for the other dominant scenarios, but we modified the distribution as follows:

- For graze and for burn, the 80 points are more concentrated in the first bin above the bar than they are in the case of grazing SB and burning KB. We did this because we do not know what species make up the RM and we do not think that these actions are as effective against the unknown mix of species as they are against SB and KB.

- For the burn/graze action, we assumed that without knowing what species make up the $\mathrm{RM}$, a combination treatment would have higher probability of being more effective at combatting a variety of species than would a graze treatment alone or a burn treatment alone. Therefore, we kept the above|below distribution at $80 \mid 20$, but we spread out the 
80 points above the bar a little more (that is, we moved more of the points upward to bins further above the bar).

\section{Quadrant 2: Starting NP Cover 20 Percent, Defoliation Level Low}

- Starting with the values in quadrant 1 (starting NP cover 80 percent, defoliation level low), we made the following modifications.

○ Rest

- $\mathrm{SB}, \mathrm{CO}$, and $\mathrm{KB}$

- Because the invasion level is high under this scenario, we proposed that the loss of NP would be slightly exacerbated; therefore, we moved five points from above the bar to the first bin below the bar.

- Because there is less room to decline, we expect (probabilistically) to see less distributional spread below the bar, so we condensed the distribution of the points to fewer bins below the bar.

- $\mathrm{RM}$

- Because the invasion level is high under this scenario, we proposed that the loss of NP would be exacerbated; therefore, we moved 10 points from above the bar to the first bin below the bar.

- Graze, Burn, Burn/Graze

- Because there is more room above the bar to grow in the case of 20 percent NP, by chance we expected to see a wider distribution spreading upward. Therefore, we moved five points from the first bin above the bar upward to the first empty bin that appeared in the 80 percent scenario.

- We did this for all active treatments, under all dominance types.

- Because there is a higher amount of invasive cover to combat with the treatment than in the 80 percent scenario, we expected a bigger positive response; therefore, we moved five points from below the bar to the highest bin with a value in the 80 percent scenario.

- We did not do this in the case of burning SB or grazing KB, to keep with the idea that these particular treatments have more dubious results when it comes to combatting these particular invasive species.

\section{Quadrant 3: Starting NP Cover 80 Percent, Defoliation Level High}

- Starting with the values in quadrant 1 (starting NP cover 80 percent, defoliation level low), we made the following modifications.

- We followed the expectation that at a starting NP cover of 80 percent, a history of high defoliation would make a year of rest less detrimental, but would have no effect on the effectiveness of the active management actions. To incorporate these ideas, we did the following:

○ Rest

- $\mathrm{SB}, \mathrm{CO}$, and $\mathrm{KB}$

- We moved 60 points from below the bar to above the bar.

- $\mathrm{RM}$

- We moved 40 points from below the bar to above the bar. 
- We moved fewer points in the case of RM dominance than we did for the other dominance types because we had already made RM less of a threat than the other dominant invasive components in the low defoliation scenario.

○ Graze, Burn, and Burn/Graze

- We simply copied and pasted the distributions from quadrant 1 (starting NP cover 80 percent, defoliation level low).

- Because we decreased the detrimental effect of rest and did not increase the effectiveness of the active treatments, rest is actually preferable to active management in some cases.

\section{Quadrant 4: Starting NP Cover 20 Percent, Defoliation Level High}

- Starting with the values in quadrant 2 (starting NP cover 20 percent, defoliation level low), we made the following modifications.

- We followed the expectation that at a NP cover of 20 percent, a history of high defoliation would make rest less detrimental and would make the active management actions more effective. To incorporate these ideas, we did the following:

$\circ$ Rest

- $\mathrm{SB}, \mathrm{CO}$, and $\mathrm{KB}$

- We moved 60 points from below the bar to above the bar.

- $\mathrm{RM}$

- We moved 40 points from below the bar to above the bar.

- The relationship between quadrant 2 and 4 (that is, NP 20, Low and NP 20, High) is the same as the relationship between quadrant 1 and 3 (that is, NP 80, Low and NP 80, High). It follows that the relationship between quadrant 1 and 2 (that is, NP 80, Low and NP 20, Low) is the same as the relationship between quadrant 3 and 4 (that is, NP 80, High and NP 20, High).

- Graze, Burn, and Burn/Graze

- All dominance scenarios (with one exception - see second bullet below)

- Moved 10 points from bins below the bar to the first bin above the bar.

- Burn/Graze combination treatment of CO

- Moved five points from below the bar to the first bin above the bar and moved five points from the second bin above the bar to the third bin above the bar.

- The reason for the different method for this scenario is because there were only 10 points below the bar to begin with, and we thought there should still be some minimum probability that the NP percent could decrease under this treatment. So, under this scenario, we still made burn/graze more effective by moving 10 points upward, but five of the points initiated from a bin higher up on the distribution ladder and were moved higher up on the ladder. 


\section{General Approach: Lower Section-Changes in Dominance}

Rather than elicit values about changes in dominance for the lower section of the spreadsheet (refer to table $10 B$ in the main text), we used the approach described below to estimate the values based on the values that were provided for changes in native cover in the upper section of the spreadsheet.

The Rules of Dominance

- $\mathrm{RM}$ is dominant if: $\mathrm{RM} /(\mathrm{SB}+\mathrm{KB}+\mathrm{RM})>=0.67$

- If $\mathrm{RM}$ is $<0.67$, then:

$\circ \mathrm{SB}$ is dominant if: $\mathrm{SB} /(\mathrm{SB}+\mathrm{KB})>=0.67$

$\circ \mathrm{KB}$ is dominant if: $\mathrm{KB} /(\mathrm{SB}+\mathrm{KB})>=0.67$

$\circ \mathrm{SB}$ and $\mathrm{KB}$ are co-dominant if: Neither is $>=0.67$

\section{Combinations of $\mathrm{SB}, \mathrm{KB}$, and RM}

There are many different combinations of the three components $(\mathrm{SB}, \mathrm{KB}, \mathrm{RM})$ that can result in each of the four dominance types ( $\mathrm{SB}, \mathrm{CO}, \mathrm{KB}, \mathrm{RM})$. To help think about the probability of shifting dominance given the different starting dominant states, management actions, and defoliation levels, we first created nine scenarios for each dominance type that reflect the different combinations of the three components (low, medium, and high) that can result in each given dominance type. These can be seen in detail in digital appendix 7a1, under the first spreadsheet entitled 'Dominance Scenarios'. In general however, the strategy was as follows:

- SB Dominance: Created nine different scenarios that are all categorized as SB dominant, but differ in their percentage of SB relative to KB [Low (67), Medium (85), and High (95)] and in their relative percentage of RM [Low (0), Medium (30), and High (60)].

- KB Dominance: Created nine different scenarios that are all categorized as KB dominant, but differ in their percentage of KB relative to SB [Low (67), Medium (85), and High (95)] and percentage of RM [Low (0), Medium (30), and High (60)].

- CO Dominance: Created nine different scenarios that are all categorized as co-dominant, but are different in the following ways. Consider an equal proportion of SB and KB (50:50), a higher proportion of SB than KB (66:34), and a lower proportion of SB than KB (34:66). Within each $\mathrm{SB} \mid \mathrm{KB}$ amount, consider a Low (0), Medium (30), and High (60) percentage of RM.

- RM Dominance: Created nine different scenarios that are all categorized as RM dominant, but are different in the following ways. Consider Low (67), Medium (85), and High (95) percentages of RM. Within each RM amount, consider an equal proportion of SB and KB (50:50), a higher proportion of SB than KB $(75: 25)$, and a lower proportion of SB than KB $(25: 75)$.

Method for Estimating Change in Dominance

We created two spreadsheets for each of the four dominance types ( $\mathrm{SB}, \mathrm{CO}, \mathrm{KB}, \mathrm{RM})$ : one under a low defoliation level and one under a high defoliation level. Therefore, we created eight spreadsheets in total (SB_Low, CO_Low, SB_Low, RM_Low, SB_High, CO_High, KB_High, RM_High). Within each spreadsheet we went through the following general steps:

(1) Given the nine different scenarios for the given dominant component, we estimated the probability of remaining in that dominance type and the probability of changing into each of the other three dominance types under rest. We based these estimates on the values provided in the upper part of the model elicitation spreadsheet; they are directly related. The rationale for the 
estimated probabilities used is annotated in comments directly within the dominance spreadsheets (digital appendix 7a1).

- Within each spreadsheet, the cells under the heading 'Probability of becoming Dominant' contain the probability of each component becoming dominant under rest and a low or high defoliation level (depending on the spreadsheet), for each dominance scenario outlined in the cells to the left.

- The cells that are color-filled are those where the estimates were made. From these cells, the remaining cells were calculated. Details of calculations can been seen by clicking on each cell.

- The spreadsheet is annotated with comments that describe in detail the rationale behind the values and what the probabilities represent with respect to the dominance scenarios to the left.

(2) We averaged the probabilities over the nine different scenarios to calculate the expected probability of each dominance type under rest.

(3) From the expected rest probabilities, we calculated the expected graze, burn, and burn/graze probabilities for each dominance type by applying the expected effects of each of these management actions on the existing SB and KB components. The management action effectiveness was extracted from the upper part of the model elicitation spreadsheet by comparing the distribution of each treatment relative to the distribution of rest for that dominance type; we used the average of the 80 percent and 20 percent scenario within each defoliation level. The probabilities for $\mathrm{CO}$ and for RM were calculated by distributing the amount that was decreased from SB and KB proportional to their starting amounts.

- Low defoliation level

- Graze decreases SB by $65 \%$ and KB by $22.5 \%$.

- Burn decreases SB by $42.5 \%$ and $\mathrm{KB}$ by $55 \%$.

- $\mathrm{BG}$ decreases SB by $65 \%$ and KB by $55 \%$.

- High defoliation level

- Graze decreases SB by $10 \%$ and $\mathrm{KB}$ by $0 \%$.

- Burn decreases SB by $0 \%$ and KB by $0 \%$.

- $\mathrm{BG}$ decreases SB by $10 \%$ and KB by $0 \%$.

Note: The effects of the management actions are relative to the effect of rest given these dominance types and defoliation levels. We are not comparing active management actions to each other, but instead to rest. The effect of rest in each dominance type was accounted for in steps 1 and 2; step 3 is where we take into account the effect of the actions relative to rest (not to each other). Although some effects may appear contradictory to the conceptual model, it is in fact consistent.

(4) We rounded the results to the nearest multiple of 5 so that they still summed to 100 , and then filled in the lower part of the model elicitation spreadsheet with these estimated values.

The final result is tied directly to the values provided in the upper part of the model elicitation spreadsheet, which is tied directly to the conceptual model. The relative pattern expected (and successfully created) between the high and low defoliation level under rest is as follows:

- Under a high defoliation level, rest is less detrimental (than under a low defoliation level) and we expect to see less increase in SB and KB (than under a low defoliation level). 
- Less increase in SB (relative to under a low defoliation level) translates into a lower probability of maintaining SB dominance if starting SB dominant and lower probability of switching to SB dominance if starting in a $\mathrm{CO}$ dominant state.

- Less increase in KB (relative to under a low defoliation level) translates into a lower probability of maintaining KB dominance if starting $\mathrm{KB}$ dominant and lower probability of switching to $\mathrm{KB}$ dominance if starting in a $\mathrm{CO}$ dominant state.

- Under a low defoliation level, rest is more detrimental (than under a high defoliation level) and we expect to see more increase in SB and KB (than under a high defoliation level).

$\circ$ More increase in SB (relative to under a high defoliation level) translates into a higher probability of maintaining SB dominance if starting SB dominant and higher probability of switching to $\mathrm{SB}$ dominance if starting in a $\mathrm{CO}$ dominant state.

- With an increase in probability of SB dominance, we expect the probability of $\mathrm{CO}$ dominance and of RM dominance to decrease.

- More increase in KB (relative to under a high defoliation level) translates into a higher probability of maintaining $\mathrm{KB}$ dominance if starting $\mathrm{KB}$ dominant and higher probability of switching to $\mathrm{KB}$ dominance if starting in a $\mathrm{CO}$ dominant state.

- With an increase in probability of KB dominance, we expect the probability of $\mathrm{CO}$ dominance and of RM dominance to decrease.

- The focus is on SB and KB; these are the species expected to increase more under a low defoliation level.

- Thus, even if the starting state is $\mathrm{CO}$ dominant or RM dominant, the expectation under a low defoliation level (relative to the high defoliation level) is that SB and $\mathrm{KB}$ will increase. Thus, we expect to see $\mathrm{SB}$ dominance and $\mathrm{KB}$ dominance increase and to see $\mathrm{CO}$ dominance and $\mathrm{RM}$ dominance decrease. 
Appendix 3b. Model elicitation spreadsheet approach and rationale for tallgrass baseline model.

\section{Process of Completing the Elicitation}

We lead a facilitated elicitation exercise to complete the model elicitation spreadsheet for the baseline tallgrass model. A USGS Science Team member proposed a set of initial values for the spreadsheet; these initial values were based on the conceptual model and guidance that was provided by the Fish and Wildlife Service (FWS) members of the Science Team. The set of values was then posed to the chosen representative and expert from the tallgrass portion of the FWS Science Team for review and consideration. The USGS team member then walked through the spreadsheet in a methodical fashion with the FWS representative, discussed the existing values, and either received confirmation on the values or instruction on how to modify the values. The completed elicitation spreadsheet is located in appendix $4 \mathrm{~b} 1$.

\section{Summary of Conceptual Model 3 (Baseline Model)}

- Management action effectiveness is invader-specific as follows:

- Smooth brome (SB): Burn within window $>$ Graze within window $>$ Defoliate $>$ Rest

- Co-dominant (CO): Burn within window $>$ Graze within window $>$ Defoliate $>$ Rest

○ Kentucky bluegrass (KB): Burn within window $>$ Graze within window $>$ Defoliate $>$ Rest

Although the relative order of the actions is the same among the invader types, the actual effectiveness of each action per invader type differs (see details under Specific Approach below).

- Management actions have different effectiveness depending on whether the defoliation level is low or high, as follows:

- Rest is less detrimental under a high defoliation level than under a low defoliation level.

- At a low native prairie (NP) cover starting state (that is, 20 percent), active management actions are more effective under a high defoliation level than under a low defoliation level.

- Management action effectiveness is not density-dependent.

$\circ$ We do not expect the effectiveness of management actions to decrease when the level of invasion increases.

- Active management actions are expected to be better than rest at all levels of invasion.

\section{Approach to Thinking about Probability}

- When distributing the 100 points for each scenario, imagine 100 different one-year time-steps.

- One can consider the same management unit and imagine how a one-year time step would look if repeated 100 different times, over 100 different years. This approach would allow one to consider, for example, different weather conditions and chance events.

- One can also consider one-year time steps in the same year for 100 different management units. This approach would allow one to consider different abiotic conditions (e.g., precipitation and soil), variability in the application of the same management action on different units, and chance events.

- Use increments no smaller than five when distributing the points. 


\section{Terminology}

- bar: Refers to the black line in each column of the spreadsheet that denotes the bin containing the NP starting state (that is, 80 percent or 20 percent).

- above the bar: Refers to the bins above the black line; points in these bins indicate that NP has remained the same or increased and the dominant invasive has remained the same or decreased.

- below the bar: Refers to the bins below the black line; points in these bins indicate that NP has decreased and the dominant invasive has increased.

General Approach: Upper Section-Changes in Native Cover

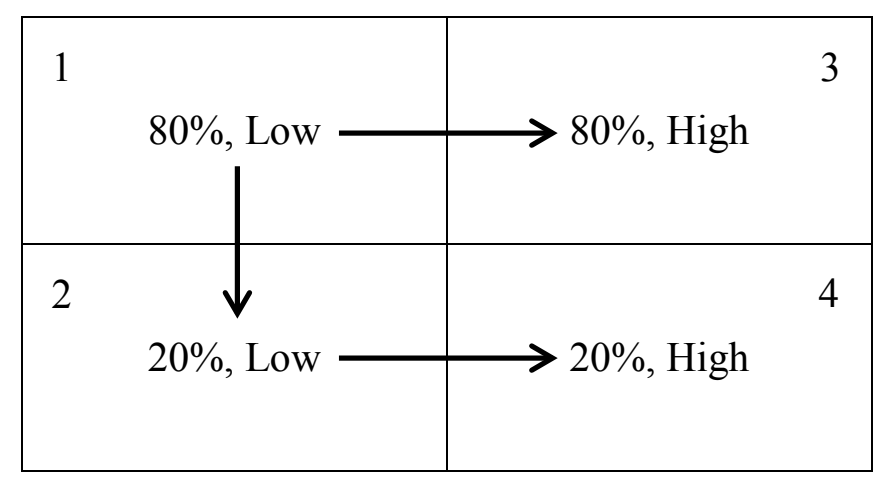

Figure 1: Simplified diagram of the first section of the elicitation spreadsheet (see table $10 \mathrm{~A}$ within the main text for the detailed spreadsheet) for changes in native cover. The diagram is divided into four quadrants, which are labeled 1-4. Quadrant 1 pertains to starting NP cover of 80 percent and low defoliation level. Quadrant 2 corresponds to starting NP cover of 20 percent and low defoliation level. Quadrant 3 represents starting NP cover of 80 percent and high defoliation level. Quadrant 4 corresponds to starting NP cover of 20 percent and high defoliation level.

Order of filling in values

(1) Set values in quadrant 1 (starting NP cover 80 percent, defoliation level low).

(2) Set values in quadrant 2 (starting NP cover 20 percent, defoliation level low) relative to values in quadrant 1.

(3) Set values in quadrant 3 (starting NP cover 80 percent, defoliation level high) relative to values in quadrant 1.

(4) Set values in quadrant 4 (starting NP cover 20 percent, defoliation level high) relative to values in quadrant 2.

\section{Specific Approach: Upper Section-Changes in Native Cover}

\section{Quadrant 1: Starting NP Cover 80 Percent, Defoliation Level Low}

- Following the conceptual model, we created the following patterns with the probability distribution above and below the bar (indicated as above|below):

○ Rest

- SB: $20 \mid 80$

- CO: $25 \mid 75$

- KB: $30 \mid 70$ 
- Remainder (RM): $50 \mid 50$

- Active management actions [graze within window (graze), burn within window (burn), defoliate]

- $\mathrm{SB}$

- Graze: $70 \mid 30$

- Burn: $80 \mid 20$

- Defoliate: $50 \mid 50$

- $\mathrm{CO}$

- Graze: $65 \mid 35$

- Burn: $80 \mid 20$

- Defoliate: $50 \mid 50$

- $\mathrm{KB}$

- Graze: $60 \mid 40$

- Burn: $80 \mid 20$

- Defoliate: $50 \mid 50$

- $\mathrm{RM}$

- Graze: $60 \mid 40$

- Burn: $60 \mid 40$

- Defoliate: $60 \mid 40$

- Within these general patterns, the way in which the points above and below the bar are distributed among the bins is different; the differences are intentional and are described below.

- We incorporated the idea that $\mathrm{SB}$ is more aggressive than $\mathrm{KB}$. We incorporated this in two different ways:

- We modified the distribution of rest for the various dominant components. SB has 80 percent of the probability below the bar, whereas KB has 70 percent below the bar, and the co-dominant has 75 percent below the bar. We made RM the least aggressive, with a $50 \mid 50$ chance of decreasing or increasing with rest.

- We modified the distribution for burning SB compared with burning KB. The amount of probability above the bar is equal in both cases (that is, 80 percent); however, we made burning slightly more effective against KB than against SB by pushing five of the points higher up above the bar in the case of KB. We used the same distribution for burning in the case of co-dominance as the case of KB dominance.

- In the case of RM dominant, we made all active management actions 60|40 (above|below).

- We made all of the active actions equally effective because the idea behind focusing treatments within the windows is based on targeting the cool-season invasive species when they are active, but when the warm-season native species are not active. Because we do not know what species make up the RM, we do not know if they are or are not active during the targeted windows. Thus, there is no reason to believe that treatments during the targeted windows are more effective against RM species than are treatments outside of the windows.

- We made management actions less effective against RM than against the cool-season invasive species because we do not know what species make up the RM and thus do not necessarily expect the treatments to damage RM species while giving a competitive boost to desirable warm-season species. We chose $60 \mid 40$ because it is better than rest $(50 \mid 50)$, 
but not better than graze in the case of KB (60|40). So as not to make it quite as good as grazing $\mathrm{KB}$, we concentrated more of the points within lower bins above the bar.

Quadrant 2: Starting NP Cover 20 Percent, Defoliation Level Low

- Starting with the values in quadrant 1 (starting NP cover 80 percent, defoliation level low), we made the following modifications.

o Rest

- $\mathrm{SB}, \mathrm{CO}, \mathrm{KB}, \mathrm{RM}$

- Split: Same split above|below

- Distribution: We condensed the distribution of points below the bar. Because there is less room to decline, we expect (probabilistically) the distribution to not be less spread out below the bar.

- Graze, Burn, Defoliate

- $\mathrm{SB}, \mathrm{CO}, \mathrm{KB}, \mathrm{RM}$

- Split: Because there is more invasive cover to combat with the treatment than in the 80 percent scenario, we expect a bigger positive response.

- Graze, Burn, and Defoliate: We moved five points from below the bar to the highest bin with a value in the 80 percent scenario.

- Distribution: Because there is more room above the bar to grow in the case of 20 percent NP, by chance we expect to see a wider distribution of points spreading upward.

o Graze and Burn: Moved five points from the first bin above the bar to the first empty bin that appeared in the 80 percent scenario.

- Defoliate: Moved five points from the first bin above the bar upward one bin. Defoliate was treated differently than graze and burn based on expert judgment.

Quadrant 3: Starting NP Cover 80 Percent, Defoliation Level High

- Starting with the values in quadrant 1 (starting NP cover 80 percent, defoliation level low), we made the following modifications.

- We followed the expectation that at a starting NP cover of 80 percent, a history of high defoliation would make a year of rest less detrimental, but would have no effect on the effectiveness of the active management actions. To incorporate these ideas, we did the following:

○ Rest

- $\mathrm{SB}, \mathrm{CO}$, and $\mathrm{KB}$

- We moved 60 points from below the bar to above the bar.

- $\mathrm{RM}$

- We moved 25 points from below the bar to above the bar.

- We moved fewer points in the case of RM dominance than we did for the other dominance types because we had already made RM less of a threat than the other dominant invasive components in the low defoliation scenario.

○ Graze, Burn, and Defoliate

- We simply copied and pasted the distributions from quadrant 1 (starting NP cover 80 percent, defoliation level low). 
- Because we decreased the detrimental effect of rest and did not increase the effectiveness of the active treatments, rest is actually preferable to active management in most cases.

Quadrant 4: Starting NP Cover 20 Percent, Defoliation Level High

- Starting with the values in quadrant 2 (starting NP cover 20 percent, defoliation level low), we made the following modifications.

- We followed the expectation that at a NP cover of 20 percent, a history of high defoliation would make rest less detrimental and would make the active management actions more effective. To incorporate these ideas, we did the following:

○ Rest

- $\mathrm{SB}, \mathrm{CO}$, and $\mathrm{KB}$

- $\mathrm{RM}$

- We moved 60 points from below the bar to above the bar.

- We moved 25 points from below the bar to above the bar.

- The relationship between quadrant 2 and 4 (that is, NP 20, Low and NP 20, High) is the same as the relationship between quadrant 1 and 3 (that is, NP 80, Low and NP 80, High). It follows that the relationship between quadrant 1 and 2 (that is, NP 80, Low and NP 20, Low) is the same as the relationship between quadrant 3 and 4 (that is, NP 80, High and NP 20, High).

- Graze, Burn, Defoliate

- Under all dominance scenarios, we increased management action effectiveness by migrating points upward. This included a mixture of modifying the split and the distribution. The manner this was done depended on the situation (mostly the existing distribution of the points); the different treatment was not an intentional difference, but one of circumstance. We use the notation ' + ' and ' - ' to refer to above and below the bar, respectively.

- $\mathrm{SB}$

- Graze and Burn

- Split: Moved five points from -2 to +1 bin

$\circ$ Distribution: Moved five points from +2 to +3 bin

- $\mathrm{CO}$ and $\mathrm{KB}$

- Graze and Burn

- Split: Moved five points from -2 to +2 bin

○ Distribution: Moved five points from +1 to +2 bin

- $\mathrm{SB}, \mathrm{CO}, \mathrm{KB}$

- Defoliate

- Split: Moved five points from -1 to +1 bin, and five points from -1 to +2 bin

- $\mathrm{RM}$

- Graze, Burn, Defoliate

○ Split: Moved 10 points from -1 to +1 bin 


\section{General Approach: Lower Section-Changes in Dominance}

Rather than elicit values about changes in dominance for the lower section of the spreadsheet (refer to table $10 B$ in the main text), we used the approach described below to estimate the values based on the values that were provided for changes in native cover in the upper section of the spreadsheet.

The Rules of Dominance

- $\mathrm{RM}$ is dominant if: $\mathrm{RM} /(\mathrm{SB}+\mathrm{KB}+\mathrm{RM})>=0.67$

- If $\mathrm{RM}$ is $<0.67$, then:

$\circ \mathrm{SB}$ is dominant if: $\mathrm{SB} /(\mathrm{SB}+\mathrm{KB})>=0.67$

$\circ \mathrm{KB}$ is dominant if: $\mathrm{KB} /(\mathrm{SB}+\mathrm{KB})>=0.67$

$\circ \mathrm{SB}$ and $\mathrm{KB}$ are co-dominant if: Neither is $>=0.67$

\section{Combinations of $\mathrm{SB}, \mathrm{KB}$, and RM}

There are many different combinations of the three components $(\mathrm{SB}, \mathrm{KB}, \mathrm{RM})$ that can result in each of the four dominance types ( $\mathrm{SB}, \mathrm{CO}, \mathrm{KB}, \mathrm{RM})$. To help think about the probability of shifting dominance given the different starting dominant states, management actions, and defoliation levels, I first created nine scenarios for each dominance type that considers the extremes (high and low) and the middle possibilities for the combinations of the three components that can still result in each given dominance type. These can be seen in detail in digital appendix 7b1, under the first spreadsheet entitled 'Dominance Scenarios'. In general however, the strategy was as follows:

- SB Dominance: Created nine different scenarios that are all categorized as SB dominant, but differ in their percentage of SB relative to KB [Low (67), Medium (85), and High (95)] and in their percentage of RM [Low (0), Medium (30), and High (60)].

- KB Dominance: Created nine different scenarios that are all categorized as KB dominant, but differ in their percentage of KB relative to SB [Low (67), Medium (85), and High (95)] and their percentage of RM [Low (0), Medium (30), and High (60)].

- CO Dominance: Created nine different scenarios that are all categorized as co-dominant, but are different in the following ways. Consider an equal proportion of SB and KB (50:50), a higher proportion of SB than KB (66:34), and a lower proportion of SB than KB (34:66). Within each $\mathrm{SB} \mid \mathrm{KB}$ amount, consider a Low (0), Medium (30), and High (60) percentage of RM.

- RM Dominance: Created nine different scenarios that are all categorized as RM dominant, but are different in the following ways. Consider Low (67), Medium (85), and High (95) percentages of RM. Within each RM amount, consider an equal proportion of SB and KB (50:50), a higher proportion of SB than KB $(75: 25)$, and a lower proportion of SB than KB $(25: 75)$.

Method for Estimating Change in Dominance

We created two spreadsheets for each of the four dominance types ( $\mathrm{SB}, \mathrm{CO}, \mathrm{KB}, \mathrm{RM})$ : one under a low defoliation level and one under a high defoliation level. Therefore, we created eight spreadsheets in total (SB_Low, CO_Low, SB_Low, RM_Low, SB_High, CO_High, KB_High, RM_High). Within each spreadsheet we went through the following general steps:

(1) Given the nine different scenarios for the given dominant component, we estimated the probability of remaining in that dominance type and the probability of changing into each of the other three dominance types under rest. We based these estimates on the values provided in the upper part of the model elicitation spreadsheet; they are directly related. The rationale for the 
estimated probabilities used is annotated in comments directly within the dominance spreadsheets (digital appendix 7b1).

- Within each spreadsheet, the cells under the heading 'Probability of becoming Dominant' contain the probability of each component becoming dominant under rest and a low or high defoliation level (depending on the spreadsheet), for each dominance scenario outlined in the cells to the left.

- The cells that are color-filled are those where the estimates were made. From these cells, the remaining cells were calculated. Details of calculations can been seen by clicking on each cell.

- The spreadsheet is annotated with comments that describe in detail the rationale behind the values and what the probabilities represent with respect to the dominance scenarios to the left.

(2) We averaged the probabilities over the nine different scenarios to calculate the expected probability of each dominance type under rest.

(3) From the expected rest probabilities, we calculated the expected graze, burn, and defoliate probabilities for each dominance type by applying the expected effects of each of these management actions on the existing SB and KB components. The management action effectiveness was extracted from the upper portion of the model elicitation spreadsheet by comparing the distribution of each treatment relative to the distribution of rest for that dominance type; we used the average of the 80 percent and 20 percent scenario within each defoliation level. The probabilities for $\mathrm{CO}$ and for RM were calculated by distributing the amount that was decreased from SB and KB proportional to their starting amounts.

- Low defoliation level

- Graze decreases SB by $52.5 \%$ and KB by $32.5 \%$.

- Burn decreases SB by $62.5 \%$ and KB by $52.5 \%$.

- Defoliate decreases SB by $32.5 \%$ and KB by $22.5 \%$.

- High defoliation level

- Graze decreases SB by $0 \%$ and KB by $0 \%$.

- Burn decreases SB by 5\% and KB by $0 \%$.

- Defoliate decreases SB by $0 \%$ and KB by $0 \%$.

Note: The effects of the management actions are relative to the effect of rest given these dominance types and defoliation levels. We are not comparing active management actions to each other, but instead to rest. The effect of rest in each dominance type was accounted for in steps 1 and 2; step 3 is where we take into account the effect of the actions relative to rest (not to each other).

(4) We rounded the results to the nearest multiple of 5 so that they still summed to 100 , and then filled in the lower part of the model elicitation spreadsheet with these estimated values.

The final result is directly tied to the values provided in the upper part of the model elicitation spreadsheet, which is directly tied to the conceptual model. The relative pattern expected (and successfully created) between the high and low defoliation level under rest is as follows:

- Under a high defoliation level, rest is less detrimental (than under a low defoliation level) and we expect to see less increase in SB and KB (than under a low defoliation level).

$\circ$ Less increase in SB (relative to under a low defoliation level) translates into a lower probability of maintaining SB dominance if starting SB dominant and lower probability of switching to SB dominance if starting in a $\mathrm{CO}$ dominant state. 
- Less increase in $\mathrm{KB}$ (relative to under a low defoliation level) translates into a lower probability of maintaining $\mathrm{KB}$ dominance if starting $\mathrm{KB}$ dominant and lower probability of switching to $\mathrm{KB}$ dominance if starting in a $\mathrm{CO}$ dominant state.

- Under a low defoliation level, rest is more detrimental (than under a high defoliation level) and we expect to see more increase in SB and KB (than under a high defoliation level).

$\circ$ More increase in SB (relative to under a high defoliation level) translates into a higher probability of maintaining SB dominance if starting SB dominant and higher probability of switching to SB dominance if starting in a $\mathrm{CO}$ dominant state.

- With an increase in probability of SB dominance, we expect the probability of $\mathrm{CO}$ dominance and of RM dominance to decrease.

- More increase in KB (relative to under a high defoliation level) translates into a higher probability of maintaining KB dominance if starting KB dominant and higher probability of switching to $\mathrm{KB}$ dominance if starting in a $\mathrm{CO}$ dominant state.

- With an increase in probability of KB dominance, we expect the probability of $\mathrm{CO}$ dominance and of RM dominance to decrease.

- The focus is on SB and KB; these are the species expected to increase more under a low defoliation level.

- Thus, even if the starting state is $\mathrm{CO}$ dominant or RM dominant, the expectation under a low defoliation level (relative to the high defoliation level) is that SB and $\mathrm{KB}$ will increase. Thus, we expect to see $\mathrm{SB}$ dominance and $\mathrm{KB}$ dominance increase and to see $\mathrm{CO}$ dominance and $\mathrm{RM}$ dominance decrease. 


\section{Appendix 4a1. Elicitation response for mixed-grass Model 3.}

[The elicitation sheet is broken into two main sections: upper and lower. The upper section focuses on the change in percent of native prairie (NP) cover, whereas the bottom section focuses on the change in the type of dominant invasive. The upper section has four quadrats: a left|right division and a top|bottom division. The left|right quadrat division is based on whether the defoliation level is low or high. The top|bottom quadrat division is based on whether the starting native cover is high ( 80 percent) or low (20 percent). Each of the four quadrats of the upper section is divided into four additional mini-quadrats; these four divisions are based on the type of dominant invasive: smooth brome (SB), co-dominant $(\mathrm{CO})$, Kentucky bluegrass $(\mathrm{KB})$, or remainder $(\mathrm{RM})$. Lastly, within each of the mini-quadrats, there are four more divisions based on the type of management action that is implemented: rest $(R)$, graze $(G)$, burn $(B)$, or burn/graze $(B / G)$. Within each of the smallest divisions of the upper section [e.g., upper section, left (low defoliation level), top ( 80 percent starting native cover), dominant invasive $\mathrm{SB}$, management action R], there are 12-13 bins; each bin represents a 5-percent range of the ending native cover (the percent range is denoted to the far left of the bins). The starting native cover is indicated by the bold horizontal line within each column of bins; this is the bar that demarks the above|below division discussed in appendix 3a. The numbers within the bins represent the probability of the ending native cover, given the starting defoliation level (low or high), starting native cover (80 percent or 20 percent), starting dominant invasive (SB, CO, KB, or RM), and the implemented management action ( $R, G, B$, or $B / G)$. The probabilities distributed among the bins sum to 100 . The lower section has two quadrats: a left and right division that is based on whether the defoliation level is low or high (this is the same left|right division as in the upper section). Similar to the upper section, the two quadrats are divided into four mini-quadrats based on the type of dominant invasive: SB, CO, KB, and RM. Also similar to the upper section, each mini-quadrat is divided into four more divisions based on the type management action that is implemented: R, G, B, or $\mathrm{B} / \mathrm{G}$. Within each of the smallest divisions of the lower section [e.g., lower section, left (low defoliation level), dominant invasive SB,

management action R], there are four bins; each bin represents the ending dominant invasive type (as denoted to the far left of the bins). The starting invasion state is indicated by the bold-outlined bin within each column of bins. The numbers within the four bins represent the probability of the ending dominant invasive type, given the starting defoliation level (low or high), starting dominant invasive type (SB, CO, KB, or RM), and the implemented management action $(\mathrm{R}, \mathrm{G}, \mathrm{B}$, or $\mathrm{B} / \mathrm{G})$. The probabilities distributed among the four bins sum to 100] 
Defoliation Level $=$ LOW

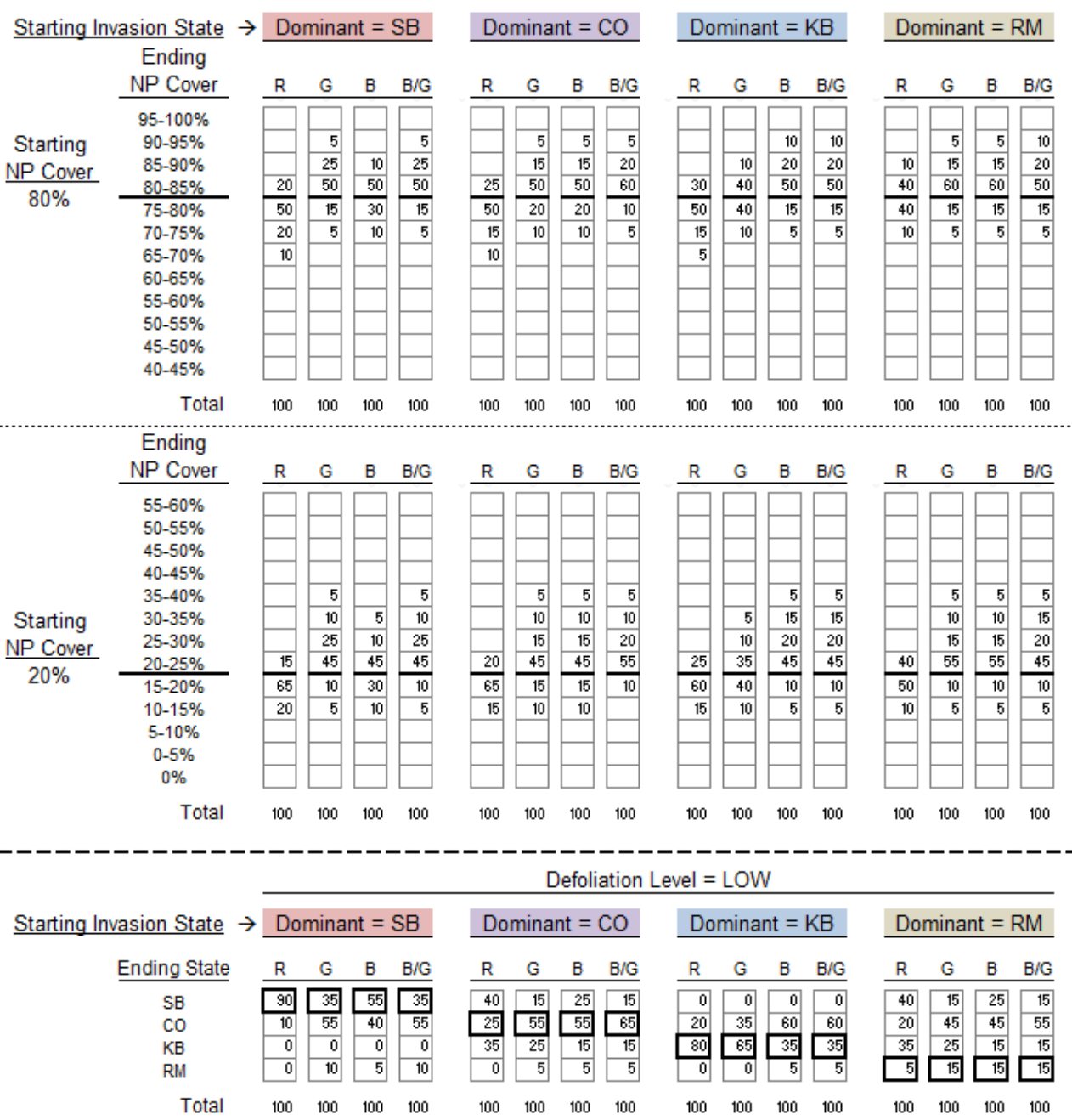

Defoliation Level $=\mathrm{HIGH}$

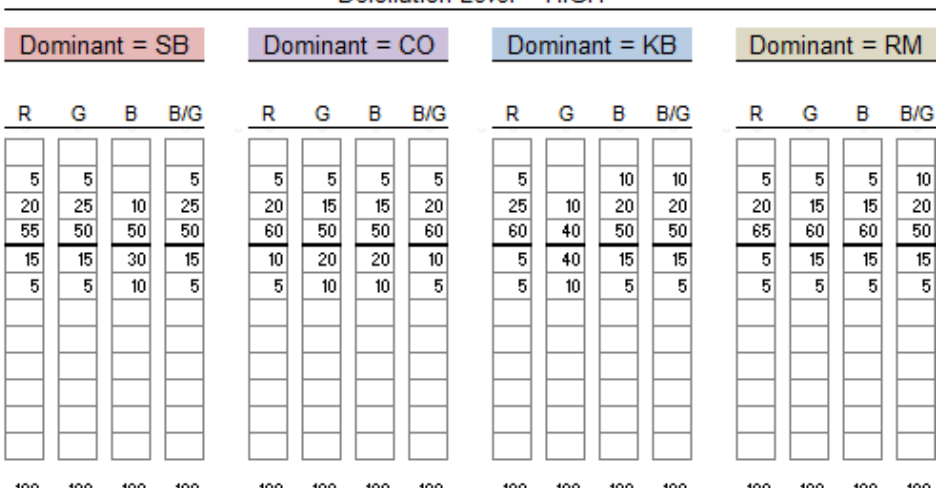

$\begin{array}{llll}100 & 100 & 100 & 100\end{array}$

$\begin{array}{llll}100 & 100 & 100 & 100\end{array}$

$100 \quad 100$

$\begin{array}{llll}100 & 100 & 100 & 100\end{array}$

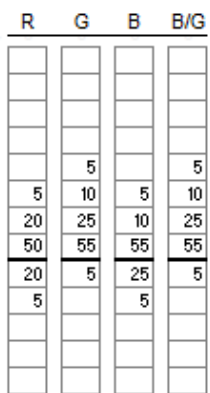

$100 \quad 100 \quad 100 \quad 100$
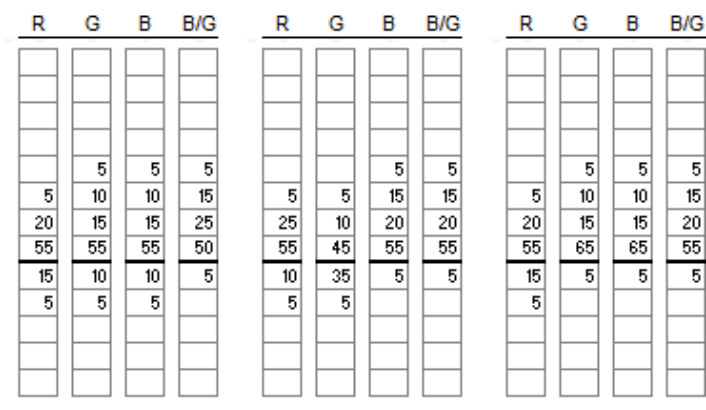

$100 \quad 100 \quad 100 \quad 100$

$\begin{array}{llll}100 & 100 & 100 & 100\end{array}$

$\begin{array}{llll}100 & 100 \quad 100 \quad 100\end{array}$

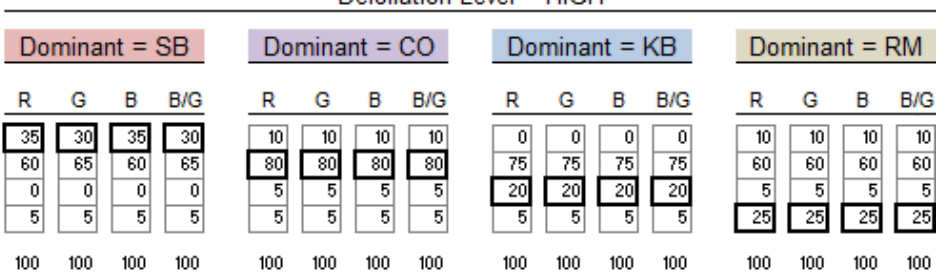


Appendix 4a2. Modification of the mixed-grass Model 3 elicitation response to provide a response for mixed-grass Model 1. [The sheet is designed the same as described in appendix 4a1]

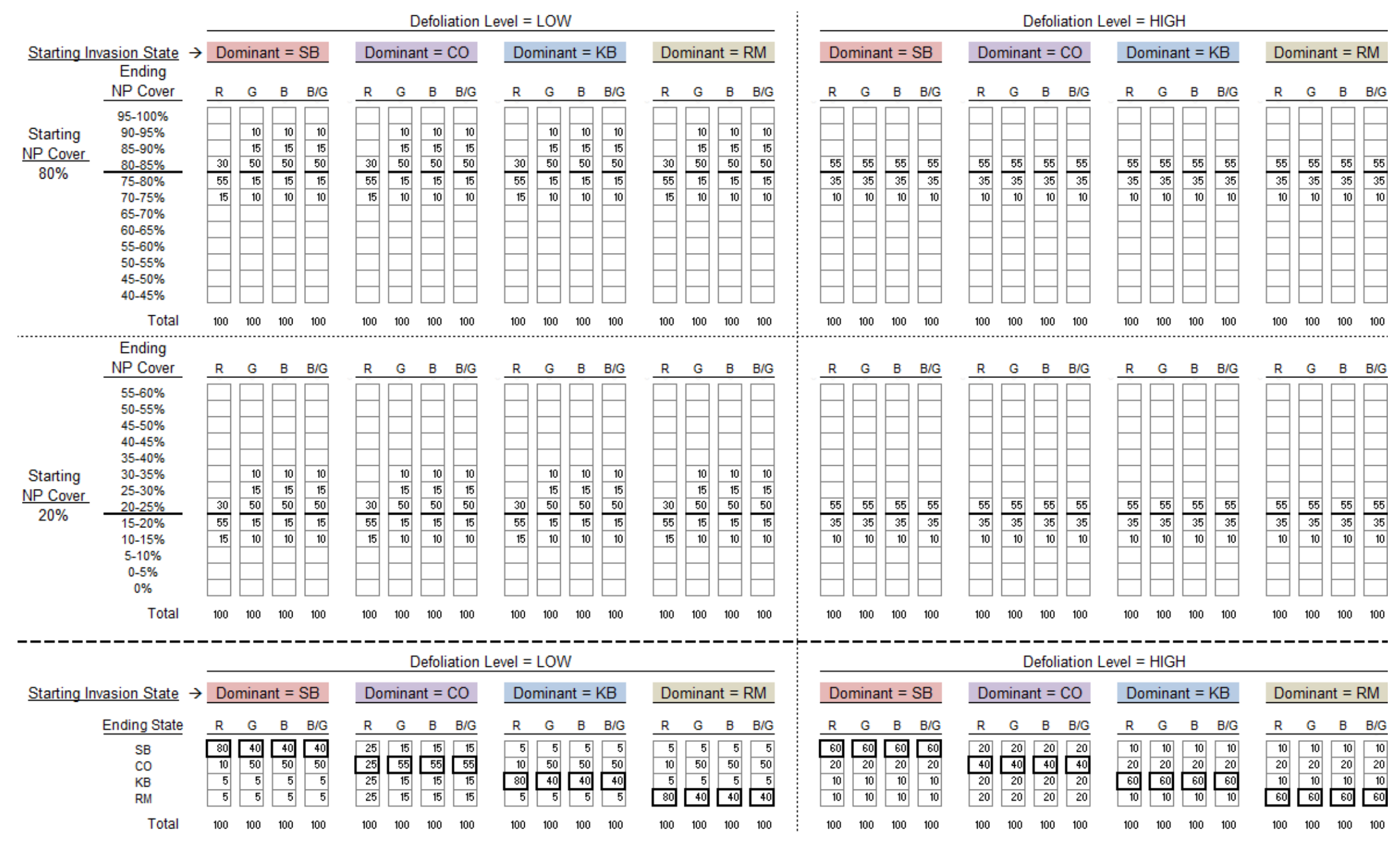


Appendix 4a3. Modification of the mixed-grass Model 3 elicitation response to provide a response for mixed-grass Model 2. [The sheet is designed the same as described in appendix 4a1]

Defoliation Level $=$ LOW

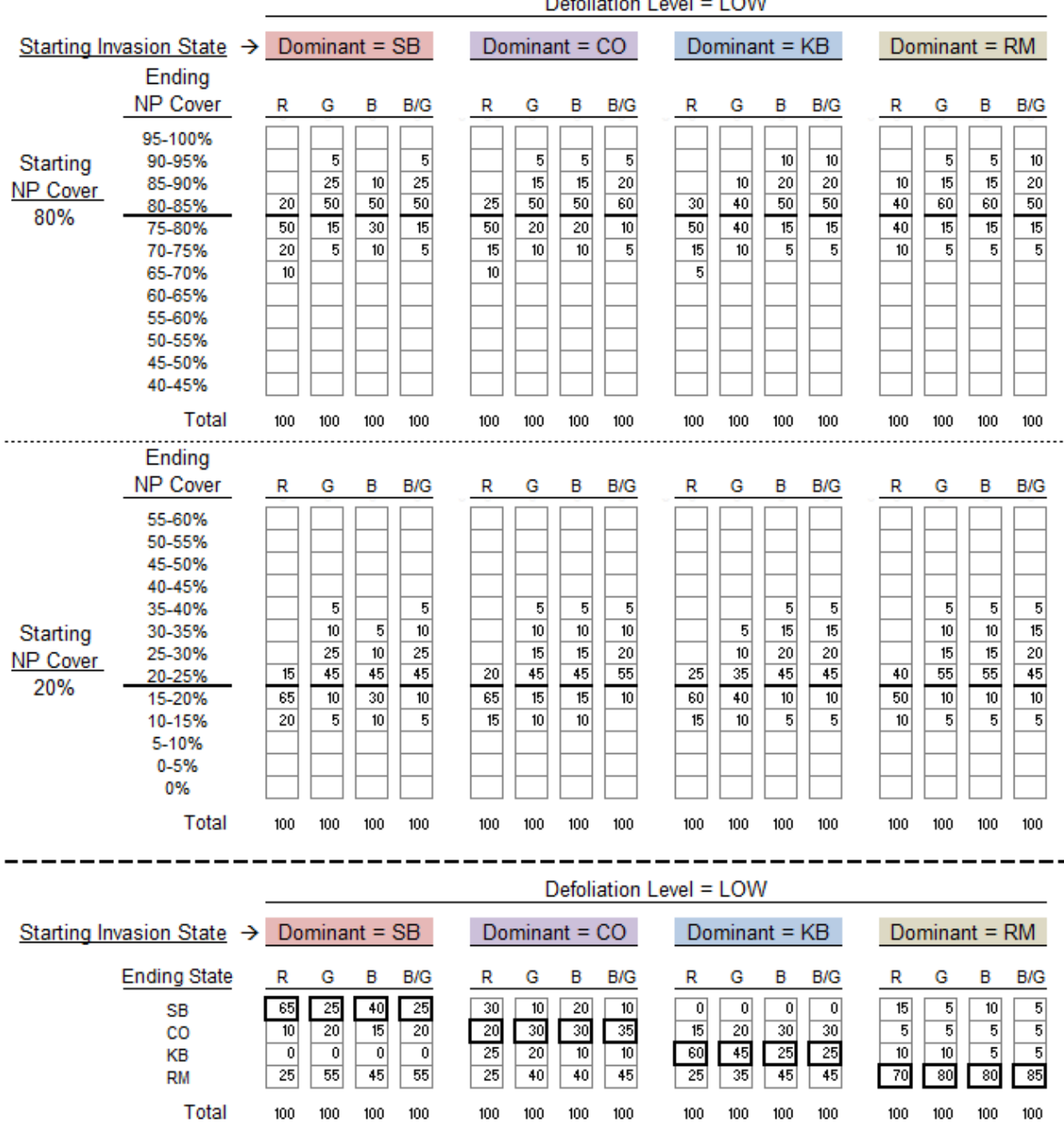

Defoliation Level $=\mathrm{HIGH}$

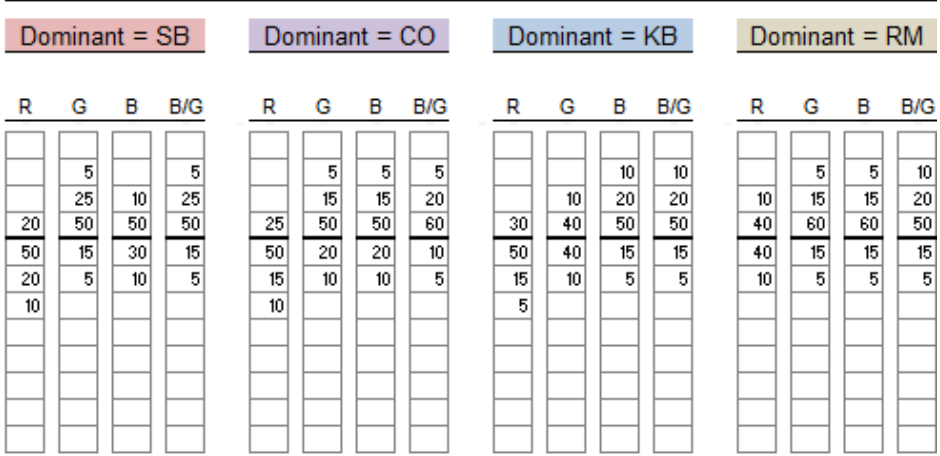

$\begin{array}{llll}100 & 100 & 100 & 100\end{array}$

$\begin{array}{llll}100 & 100 & 100 & 100\end{array}$

$100 \quad 100 \quad 100 \quad 100$

$\begin{array}{llll}100 & 100 & 100 & 100\end{array}$

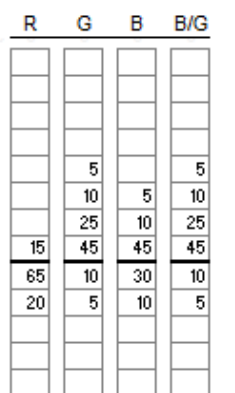

$100 \quad 100 \quad 100 \quad 100$

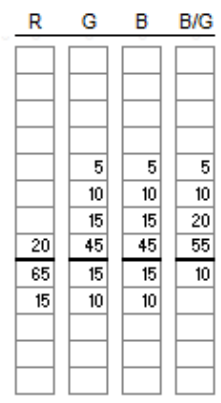

$100 \quad 100 \quad 100 \quad 100$
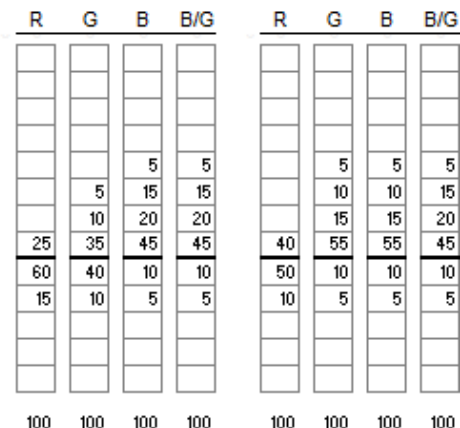

Defoliation Level $=\mathrm{HIGH}$

Dominant $=\mathrm{SB} \quad$ Dominant $=\mathrm{CO} \quad$ Dominant $=\mathrm{KB} \quad$ Dominant $=\mathrm{RM}$

\begin{tabular}{|c|c|c|c|c|c|c|c|c|c|c|c|c|c|c|c|}
\hline$n$ & G & $B$ & $B / G$ & $\mathrm{R}$ & $G$ & B & $\mathrm{B} / \mathrm{G}$ & R & $G$ & B & $\mathrm{B} / \mathrm{G}$ & $R$ & G & $B$ & $\mathrm{~B} / \mathrm{G}$ \\
\hline 65 & \begin{tabular}{|l|}
25 \\
\end{tabular} & \begin{tabular}{|l|}
40 \\
\end{tabular} & \begin{tabular}{|l|}
25 \\
\end{tabular} & 30 & 10 & 20 & 10 & 0 & 0 & 0 & 0 & 15 & 5 & 10 & 5 \\
\hline 10 & 20 & 15 & \begin{tabular}{|l|}
20 \\
\end{tabular} & 20 & 30 & 30 & 35 & 15 & 20 & 30 & 30 & 5 & 5 & 5 & 5 \\
\hline 0 & 0 & 0 & 0 & 25 & 20 & 10 & 10 & 60 & 45 & 25 & 2 & 10 & 10 & 5 & 5 \\
\hline 25 & \begin{tabular}{|c|}
55 \\
\end{tabular} & \begin{tabular}{|l|}
45 \\
\end{tabular} & \begin{tabular}{|l}
55 \\
\end{tabular} & 25 & \begin{tabular}{|l|}
40 \\
\end{tabular} & 40 & 45 & & 35 & 45 & 45 & 10 & \begin{tabular}{|l}
80 \\
\end{tabular} & \begin{tabular}{|l}
80 \\
\end{tabular} & \begin{tabular}{|l}
85 \\
\end{tabular} \\
\hline
\end{tabular}

$\begin{array}{llll}100 & 100 & 100 & 100\end{array}$ 
Appendix 4a4. Modification of the mixed-grass Model 3 elicitation response to provide a response for mixed-grass Model 4.

[The sheet is designed the same as described in appendix 4a1]

Defoliation Level $=$ LOW

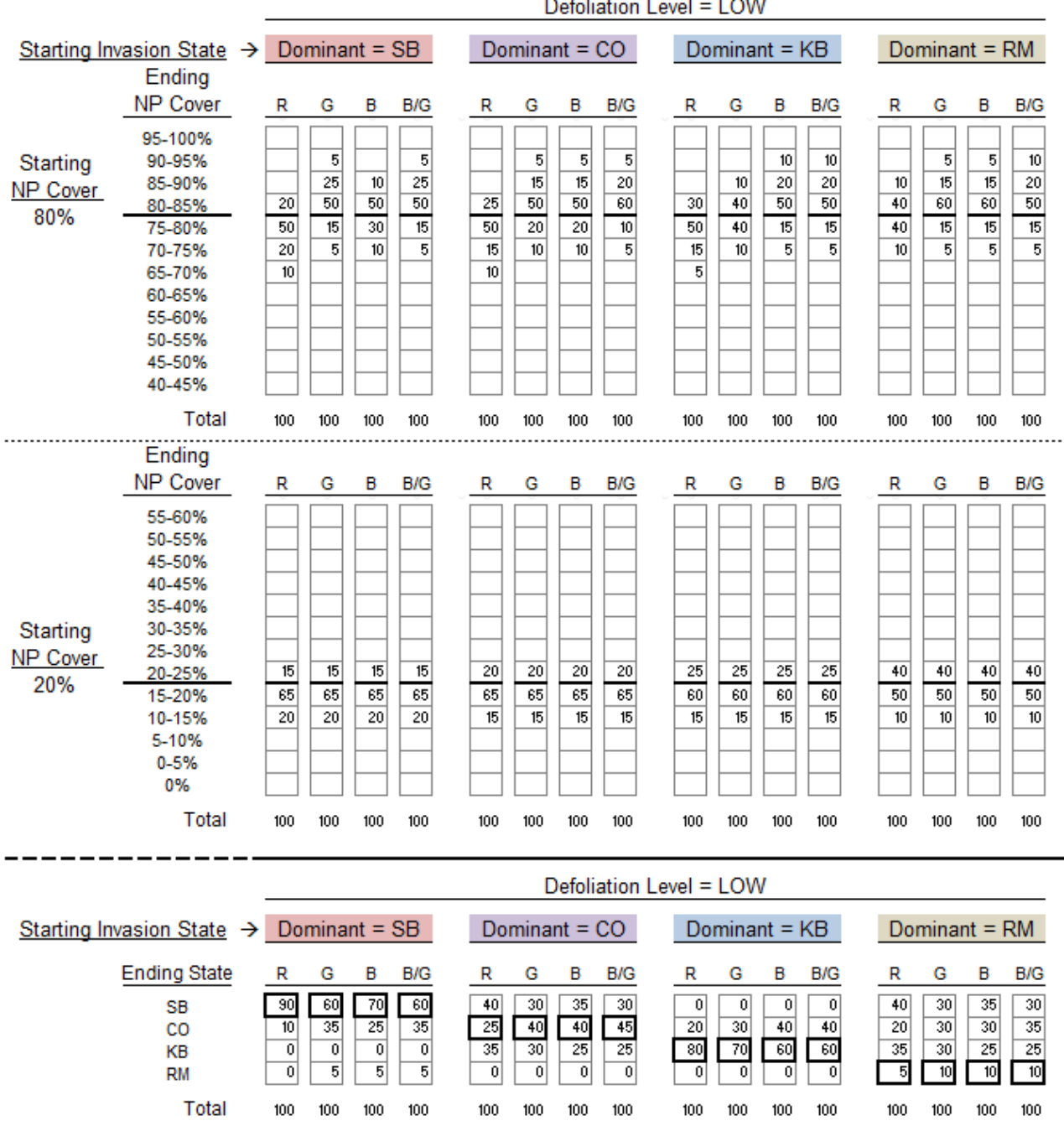

Defoliation Level $=\mathrm{HIGH}$

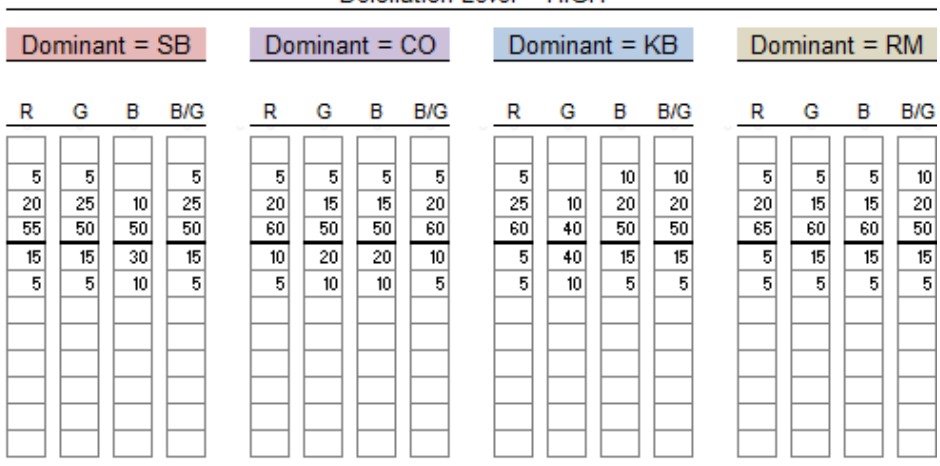

$100 \quad 100 \quad 100 \quad 100$

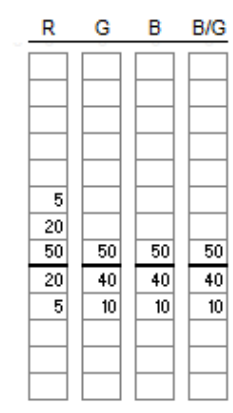

$100 \quad 100 \quad 100 \quad 100$

$100 \quad 100 \quad 100 \quad 100$

$100 \quad 100 \quad 100 \quad 100$

$100 \quad 100 \quad 100 \quad 100$
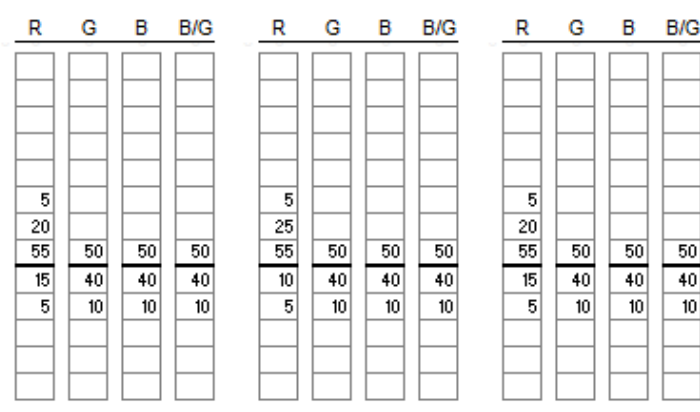

$100 \quad 100 \quad 100 \quad 100$

$100 \quad 100 \quad 100 \quad 100$

$100 \quad 100 \quad 100 \quad 100$

\begin{tabular}{|c|c|c|c|c|c|c|c|c|c|c|c|c|c|c|c|}
\hline \multicolumn{4}{|c|}{ Dominant $=\mathrm{SB}$} & \multicolumn{4}{|c|}{ Dominant $=\mathrm{CO}$} & \multicolumn{4}{|c|}{ Dominant $=\mathrm{KB}$} & \multicolumn{4}{|c|}{ Dominant $=\mathrm{RM}$} \\
\hline R & $G$ & B & $B / G$ & $R$ & G & B & $\mathrm{B} / \mathrm{G}$ & $R$ & G & B & $\mathrm{B} / \mathrm{G}$ & $R$ & G & B & $B / G$ \\
\hline \begin{tabular}{|c|}
35 \\
\end{tabular} & \begin{tabular}{|c|}
35 \\
\end{tabular} & 35 & $\longdiv { 3 5 }$ & 10 & 10 & & $\overline{10}$ & 0 & 0 & 0 & 0 & 10 & 10 & 10 & $\overline{10}$ \\
\hline \begin{tabular}{|c|}
60 \\
\end{tabular} & \begin{tabular}{|l|l}
60 \\
\end{tabular} & 60 & 60 & \begin{tabular}{|l|}
80 \\
\end{tabular} & \begin{tabular}{|l|}
80 \\
\end{tabular} & \begin{tabular}{|l|}
80 \\
\end{tabular} & \begin{tabular}{|l|}
80 \\
\end{tabular} & \begin{tabular}{|l}
75 \\
\end{tabular} & \begin{tabular}{|l|}
75 \\
\end{tabular} & \begin{tabular}{|l|}
75 \\
\end{tabular} & \begin{tabular}{|l}
75 \\
\end{tabular} & \begin{tabular}{|l|}
60 \\
\end{tabular} & 60 & 60 & \begin{tabular}{|l|}
60 \\
\end{tabular} \\
\hline$\frac{0}{5}$ & 0 & 0 & 0 & 5 & 5 & 5 & 5 & \begin{tabular}{|l|}
20 \\
\end{tabular} & $\sqrt{20}$ & \begin{tabular}{|l|}
20 \\
\end{tabular} & 20 & 5 & 5 & 5 & 5 \\
\hline 5 & 5 & 5 & 5 & 5 & 5 & & 5 & & & & 5 & & & & \begin{tabular}{|l|}
25 \\
\end{tabular} \\
\hline
\end{tabular}


Appendix 4b1. Elicitation response for tallgrass Model 3.

[The sheet is designed the same as described in appendix 4a1 for the mixed-grass model, with one exception; the management actions for tallgrass are rest $(R)$, graze within window $(G)$, burn within window $(B)$, and defoliate outside of window (D)]

Defoliation Level $=\mathrm{LOW}$

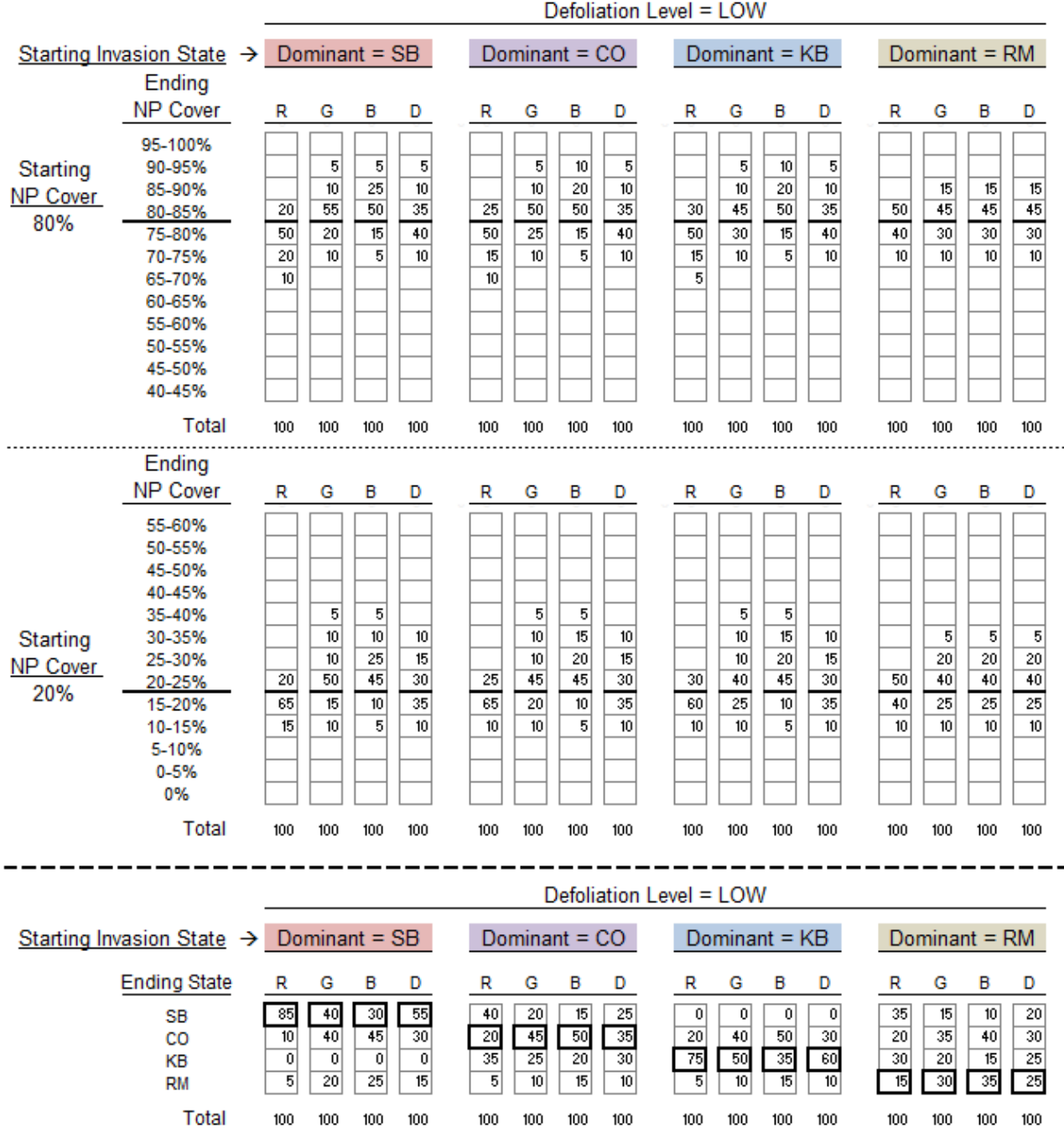

Defoliation Level $=\mathrm{HIGH}$

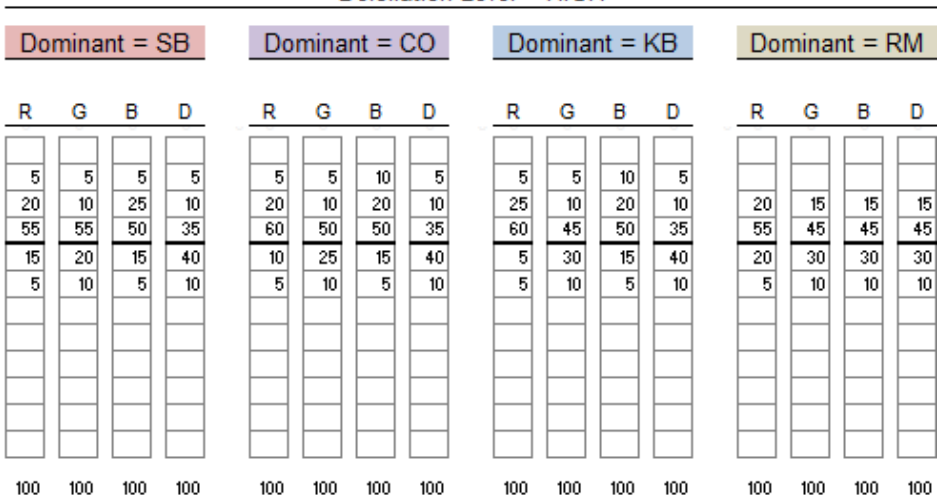

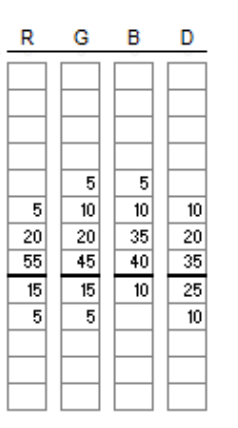
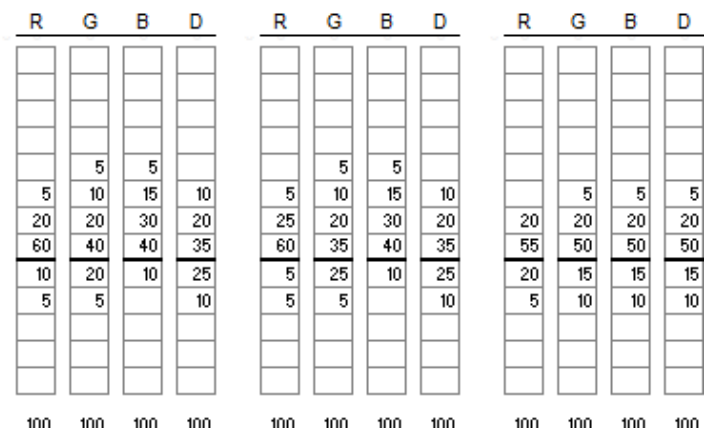

$100 \quad 100 \quad 100 \quad 100$

$100 \quad 100 \quad 100 \quad 100$

$100 \quad 100 \quad 100 \quad 100$

$100 \quad 100 \quad 100 \quad 100$

Defoliation Level $=\mathrm{HIGH}$

Dominant $=\mathrm{SB}$ Dominant $=\mathrm{CO}$ Dominant $=\mathrm{KB}$ Dominant $=\mathrm{RM}$

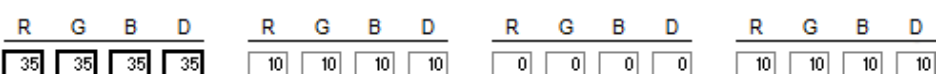

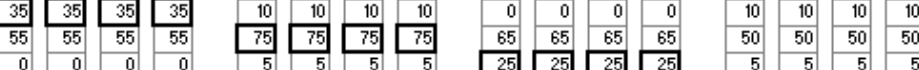

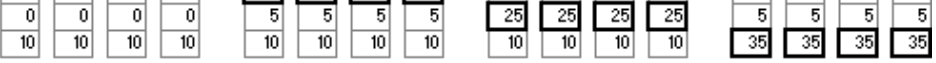

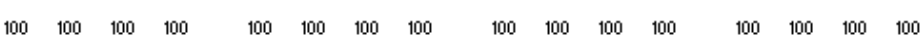


Appendix 4b2. Modification of the tallgrass Model 3 elicitation response to provide a response for tallgrass Model 1.

[The sheet is designed the same as described in appendix 4a1 for the mixed-grass model, with one exception; the management actions for tallgrass are rest $(R)$, graze within window $(G)$, burn within window $(B)$, and defoliate outside of window (D)]

\begin{tabular}{|c|c|c|c|c|c|c|c|c|c|c|c|c|c|c|c|c|c|c|c|c|c|c|c|c|c|c|c|c|c|c|c|c|c|}
\hline \multirow{3}{*}{\multicolumn{2}{|c|}{$\begin{array}{r}\text { Starting Invasion State } \\
\text { Ending } \\
\text { NP Cover }\end{array}$}} & \multicolumn{16}{|c|}{ Defoliation Level = LOW } & \multicolumn{16}{|c|}{ Defoliation Level $=\mathrm{HIGH}$} \\
\hline & & \multicolumn{4}{|c|}{ Dominant $=\mathrm{SB}$} & \multicolumn{4}{|c|}{ Dominant $=\mathrm{CO}$} & \multicolumn{4}{|c|}{ Dominant $=\mathrm{KB}$} & \multicolumn{4}{|c|}{ Dominant $=\mathrm{RM}$} & \multicolumn{4}{|c|}{ Dominant $=$ SB } & \multicolumn{4}{|c|}{ Dominant $=\mathrm{CO}$} & \multicolumn{4}{|c|}{ Dominant $=\mathrm{KB}$} & \multicolumn{4}{|c|}{ Dominant $=\mathrm{RM}$} \\
\hline & & $\mathrm{R}$ & G & $\mathrm{B}$ & $\mathrm{D}$ & $R$ & G & $\mathrm{B}$ & $\mathrm{D}$ & $R$ & G & $\mathrm{B}$ & $\mathrm{D}$ & $\mathrm{R}$ & G & $B$ & $D$ & $R$ & G & $\mathrm{B}$ & $\mathrm{D}$ & $R$ & G & $\mathrm{B}$ & $D$ & $R$ & G & $\mathrm{B}$ & $\mathrm{D}$ & $\mathrm{R}$ & G & $\mathrm{B}$ & D \\
\hline \multirow{12}{*}{$\begin{array}{c}\text { Starting } \\
\text { NP Cover } \\
80 \%\end{array}$} & $95-100 \%$ & \begin{tabular}{|l|l} 
\\
\end{tabular} & $\square$ & 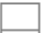 & & $\square$ & a & $\square$ & 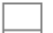 & $\square$ & $\square$ & \begin{tabular}{|l|l} 
\\
\end{tabular} & & \begin{tabular}{|l|l} 
\\
\end{tabular} & $\square$ & 1 & & $\square$ & $\square$ & \begin{tabular}{|l|l} 
\\
\end{tabular} & - & $\square$ & $\square$ & \begin{tabular}{|l|l} 
\\
\end{tabular} & $\square$ & $\square$ & $\square$ & $\square$ & $\square$ & 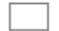 & \begin{tabular}{|l|l} 
\\
\end{tabular} & \begin{tabular}{|l|l|l|} 
\\
\end{tabular} & $\square$ \\
\hline & $90-95 \%$ & $\square$ & 10 & 10 & 10 & 4 & 10 & 10 & 10 & $\square$ & 10 & 10 & 10 & \begin{tabular}{|l|} 
\\
\end{tabular} & 10 & 10 & 10 & $\square$ & $\square$ & \begin{tabular}{|l|l|l} 
\\
\end{tabular} & -1 & $\square$ & $\square$ & \begin{tabular}{|l|} 
\\
\end{tabular} & $\square$ & $\square$ & \begin{tabular}{|l|l|l|l} 
& \\
\end{tabular} & \begin{tabular}{|l|} 
\\
\end{tabular} & \begin{tabular}{|l|} 
\\
\end{tabular} & $\square$ & \begin{tabular}{|l|l|} 
\\
\end{tabular} & \begin{tabular}{|l|l} 
\\
\end{tabular} & $\square$ \\
\hline & $85-90 \%$ & 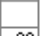 & 15 & 15 & 15 & 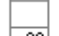 & 15 & 15 & 15 & $\square$ & 15 & 15 & 15 & 10 & 15 & 15 & 15 & $=$ & & $=F$ & & $=$ & & & & $=1$ & & & & & & & \\
\hline & $\frac{80-85 \%}{75-80 \%}$ & $\frac{30}{55}$ & $\frac{40}{25}$ & 40 & 40 & $\frac{30}{55}$ & $\frac{40}{25}$ & 40. & 40 & $\frac{30}{55}$ & 400 & 40 & 40 & \begin{tabular}{|l|}
30 \\
55
\end{tabular} & $\frac{40}{25}$ & $\frac{40}{25}$ & $\frac{40}{25}$ & $\frac{55}{35}$ & $\frac{55}{35}$ & \begin{tabular}{|l|}
35 \\
35 \\
\end{tabular} & $\frac{55}{35}$ & $\frac{55}{35}$ & $\frac{55}{35}$ & $\frac{55}{35}$ & $\frac{55}{5.5}$ & $\frac{55}{35}$ & $\frac{55}{5.5}$ & 55 & $\frac{55}{5.5}$ & 55 & 55 & 55 & 55 \\
\hline & $\begin{array}{l}75-80 \% \\
70-75 \%\end{array}$ & \begin{tabular}{|l|}
55 \\
15
\end{tabular} & $\begin{array}{r}25 \\
10\end{array}$ & \begin{tabular}{|l|}
25 \\
10
\end{tabular} & 25 & $\begin{array}{r}55 \\
15\end{array}$ & $\begin{array}{r}25 \\
10\end{array}$ & \begin{tabular}{|c|}
25 \\
10
\end{tabular} & 25 & $\begin{array}{r}55 \\
15\end{array}$ & $\begin{array}{r}25 \\
10\end{array}$ & \begin{tabular}{|r|}
25 \\
10
\end{tabular} & $\begin{array}{r}25 \\
10\end{array}$ & \begin{tabular}{|r|}
55 \\
15 \\
\end{tabular} & $\frac{25}{10}$ & \begin{tabular}{|c|}
25 \\
10
\end{tabular} & $\frac{25}{10}$ & $\begin{array}{r}35 \\
10\end{array}$ & \begin{tabular}{|r|}
35 \\
10
\end{tabular} & \begin{tabular}{|r|}
35 \\
10 \\
\end{tabular} & \begin{tabular}{|c|}
35 \\
10
\end{tabular} & $\begin{array}{r}\frac{35}{10} \\
\end{array}$ & 年5 & \begin{tabular}{|c|}
35 \\
10
\end{tabular} & $\begin{array}{r}355 \\
10\end{array}$ & $\frac{35}{10}$ & $\frac{35}{10}$ & \begin{tabular}{|c|}
35 \\
10 \\
\end{tabular} & $\begin{array}{r}\frac{35}{10} \\
\end{array}$ & $\frac{35}{10}$ & $\frac{35}{10}$ & $\begin{array}{c}35 \\
10\end{array}$ & $\begin{array}{r}35 \\
10\end{array}$ \\
\hline & $65-70 \%$ & $\square$ & $\square$ & ( & 0 & 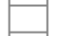 & a & \begin{tabular}{|c|}
10 \\
\end{tabular} & 10 & 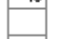 & $\square$ & \begin{tabular}{|c|} 
\\
\end{tabular} & 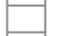 & \begin{tabular}{|l|} 
\\
\end{tabular} & 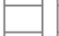 & \begin{tabular}{|l|} 
\\
\end{tabular} & $\square$ & +10 & \begin{tabular}{|l|} 
\\
\end{tabular} & \begin{tabular}{|l|l|} 
\\
\end{tabular} & \begin{tabular}{|l|l} 
\\
\end{tabular} & + & $\because$ & 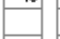 & 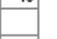 & $n$ & 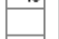 & \begin{tabular}{|l|} 
\\
\end{tabular} & 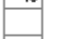 & 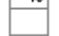 & $a$ & - & 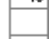 \\
\hline & $60-65 \%$ & $\square$ & $\square$ & $\square$ & & $\square$ & $\square$ & & & $\square$ & $\square_{1}$ & & & $\square$ & & & & & & & & & & & & & & & & & & & $\square$ \\
\hline & $55-60 \%$ & 员 & - & 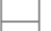 & -1 & -1 & & \begin{tabular}{|l} 
\\
\end{tabular} & -1 & - & $\square$ & 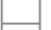 & -1 & \begin{tabular}{|l} 
\\
\end{tabular} & \begin{tabular}{|l} 
\\
\end{tabular} & \begin{tabular}{|c|c|c|} 
& \\
\end{tabular} & 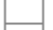 & $\square$ & 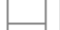 & \begin{tabular}{|l|l} 
\\
\end{tabular} & \begin{tabular}{|l} 
\\
\end{tabular} & 4 & - & \begin{tabular}{|l} 
\\
\end{tabular} & \begin{tabular}{|l} 
\\
\end{tabular} & - & 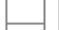 & 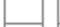 & -1 & - & & & - \\
\hline & $50-55 \%$ & $\square$ & - & \begin{tabular}{|l} 
\\
\end{tabular} & -1 & -1 & -1 & \begin{tabular}{|l} 
\\
\end{tabular} & \begin{tabular}{|l} 
\\
\end{tabular} & -1 & $\mathrm{D}^{\prime}$ & ( & -1 & \begin{tabular}{|l} 
\\
\end{tabular} & \begin{tabular}{|l|l} 
\\
\end{tabular} & \begin{tabular}{|l|l} 
\\
\end{tabular} & 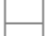 & - & -1 & \begin{tabular}{|l} 
\\
\end{tabular} & 品 & $\square$ & - & \begin{tabular}{|l} 
\\
\end{tabular} & 品 & - & \begin{tabular}{|l} 
\\
\end{tabular} & \begin{tabular}{|l} 
\\
\end{tabular} & - & - & \begin{tabular}{|l} 
\\
\end{tabular} & \begin{tabular}{|c|c|} 
\\
\end{tabular} & 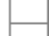 \\
\hline & $\%$ & - & - & 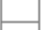 & \begin{tabular}{|l} 
\\
\end{tabular} & -1 & -1 & \begin{tabular}{|l} 
\\
\end{tabular} & 国 & 员 & - & \begin{tabular}{|c|c} 
\\
\end{tabular} & -1 & & \begin{tabular}{|c|c|c|} 
\\
\end{tabular} & - & -1 & & & | & & & & & - & & - & \begin{tabular}{|l} 
\\
\end{tabular} & -1 & -1 & 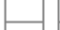 & & $\Delta$ \\
\hline & $40-45 \%$ & $\square$ & 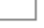 & $\square$ & 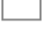 & $\square$ & 工 & - & $\square$ & & $\square$ & $\square$ & 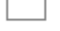 & & $\square$ & \begin{tabular}{|l} 
\\
\end{tabular} & $\square$ & -1 & ए & - & $\square$ & 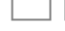 & $\square$ & $\square$ & $\square$ & $\square$ & - & \begin{tabular}{|l}
$\square$ \\
\end{tabular} & - & $\square$ & - & $\square$ & $\square$ \\
\hline & Total & 100 & 100 & 100 & 100 & 100 & 100 & 100 & 100 & 100 & 100 & 100 & 100 & 100 & 100 & 100 & 100 & 100 & 100 & 100 & 100 & 100 & 100 & 100 & 100 & 100 & 100 & 100 & 100 & 100 & 100 & 100 & 100 \\
\hline \multirow{15}{*}{$\begin{array}{c}\text { Starting } \\
\text { NP Cover } \\
20 \%\end{array}$} & Ending & & & & & & & & & & & & & & & & & & & & & & & & & & & & & & & & \\
\hline & NP Cover & $\mathrm{R}$ & G & B & D & $\mathrm{R}$ & G & $\mathrm{B}$ & D & $\mathrm{R}$ & G & $\mathrm{B}$ & D & $\mathrm{R}$ & G & $\mathrm{B}$ & D & $\mathrm{R}$ & G & B & D & $\mathrm{R}$ & G & B & D & $\mathrm{R}$ & G & B & D & $\mathrm{R}$ & G & $\mathrm{B}$ & D \\
\hline & $55-60 \%$ & $\square$ & $\square$ & $\square$ & 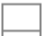 & $\square$ & 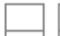 & \begin{tabular}{|l|} 
\\
\end{tabular} & 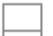 & $\square$ & $\square$ & $\square$ & & - & \begin{tabular}{|l|} 
\\
\end{tabular} & \begin{tabular}{|l|} 
\\
\end{tabular} & $\square$ & 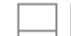 & 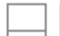 & 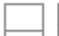 & & 4 & - & $\square$ & & -1 & 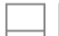 & - & & & - & 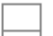 & $\square$ \\
\hline & $50-$ & $\square$ & $\square$ & $\square$ & $\square$ & $\square$ & 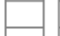 & \begin{tabular}{|l|} 
\\
\end{tabular} & -1 & $\square$ & $\square$ & $\square$ & $\square$ & - & $\square$ & $\square$ & $\square$ & $\square$ & $\square$ & $\square$ & $\square$ & $\square$ & $\square$ & $\square$ & E & E & $\square$ & \begin{tabular}{|l|} 
\\
\end{tabular} & $\square$ & - & 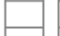 & - & $\square$ \\
\hline & $45-50 \%$ & $\square$ & $\square$ & $\square$ & & $\square$ & - & \begin{tabular}{|l} 
\\
\end{tabular} & -1 & $\square$ & \begin{tabular}{|l|} 
\\
\end{tabular} & $\square$ & & - & \begin{tabular}{|l|l} 
\\
\end{tabular} & \begin{tabular}{|l|} 
\\
\end{tabular} & $\square$ & 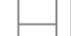 & $\square$ & \begin{tabular}{|l} 
\\
\end{tabular} & & 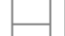 & 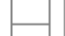 & \begin{tabular}{|l} 
\\
\end{tabular} & $\square$ & - & & & $\square$ & - & \begin{tabular}{|l} 
\\
\end{tabular} & $\square$ & $\square$ \\
\hline & $\%$ & $\square$ & $\square$ & $\square$ & - & $\square$ & 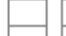 & \begin{tabular}{|l} 
\\
\end{tabular} & $\square$ & $\square$ & \begin{tabular}{|l|} 
\\
\end{tabular} & \begin{tabular}{|l} 
\\
\end{tabular} & $\square$ & 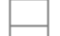 & $\square$ & $\square$ & $\square$ & $\square$ & $\square$ & $\square$ & $\square$ & $\square$ & $\square$ & $\square$ & $\square$ & $\square$ & \begin{tabular}{|l} 
\\
\end{tabular} & \begin{tabular}{|l|} 
\\
\end{tabular} & $\square$ & 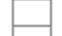 & $\square$ & $\square$ & $\square$ \\
\hline & & 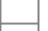 & 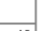 & 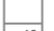 & & - & & \begin{tabular}{|l|} 
\\
\end{tabular} & & $\square$ & 1 & $\mathrm{C}_{0}$ & & - & 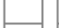 & 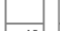 & & 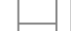 & - & - & 品 & -1 & & \begin{tabular}{|l} 
\\
\end{tabular} & & 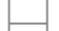 & & & & & & - & $\square$ \\
\hline & $30-35 \%$ & $\square$ & 10 & 10 & 10 & $\square$ & 10 & 10 & 10 & 1 & 10 & \begin{tabular}{|l|}
10 \\
\end{tabular} & 10 & 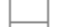 & 10 & 10 & 10 & + & 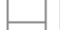 & $\square$ & - & -1 & -1 & \begin{tabular}{|l} 
\\
\end{tabular} & $\square$ & -1 & 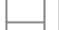 & 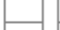 & -1 & - & $\square$ & \begin{tabular}{|l|} 
\\
\end{tabular} & $\square$ \\
\hline & $25-30 \%$ & 30 & $\begin{array}{r}15 \\
40\end{array}$ & $\frac{15}{40}$ & \begin{tabular}{|l|}
15 \\
40
\end{tabular} & 30 & $\frac{15}{40}$ & \begin{tabular}{|r|}
15 \\
40
\end{tabular} \mid & $\frac{15}{40}$ & 30 & $\frac{15}{40}$ & \begin{tabular}{|r|}
15 \\
40
\end{tabular} & $\frac{15}{40}$ & 30 & $\frac{15}{40}$ & $\frac{15}{40}$ & 15 & & & & & & & & & & & & & & & & \\
\hline & $20-25$ & 30 & 40 & 40 & 40 & 30 & 40. & 40 & 40 & 30 & 40 & 40 & 40 & 30 & 40 & 40 & 40 & 55 & 55 & 55 & 55 & 55 & 55 & 55 & 55 & 55 & 55 & 55 & 55 & 55 & 55 & 55 & 55 \\
\hline & $15-20 \%$ & 55 & 25 & 25 & 25 & 55 & 25 & 25 & 25 & 55 & \begin{tabular}{|l|}
25 \\
\end{tabular} & $25 \mid$ & 25 & 55 & 25 & 25 & 25 & 35 & 35 & 35 & 35 & 35 & 35 & \begin{tabular}{|l|}
35 \\
\end{tabular} & 35 & 35 & 35 & \begin{tabular}{|l|}
35 \\
\end{tabular} & 35 & 35 & \begin{tabular}{|l|}
35 \\
\end{tabular} & 35 & 35 \\
\hline & $10-15 \%$ & 15 & 10 & 10 & 10 & 15 & 10 & 10 & 10 & 15 & $10 \mid$ & $10 \mid$ & 10 & 15 & 10 & 10 & 10 & 10 & 10 & 10 & $10 \mid$ & 10 & 10 & 10 & 10 & 10 & 10 & \begin{tabular}{|l|}
10 \\
\end{tabular} & 10 & 10 & \begin{tabular}{|l|}
10 \\
\end{tabular} & 10 & 10 \\
\hline & $5-10 \%$ & 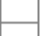 & -1 & $\mid$ & \begin{tabular}{|l} 
\\
\end{tabular} & -1 & -1 & $a^{-1}$ & \begin{tabular}{|l} 
\\
\end{tabular} & -1 & \begin{tabular}{|l|} 
\\
\end{tabular} & $\square_{1}$ & -1 & 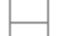 & $\square$ & $\square$ & \begin{tabular}{|l} 
\\
\end{tabular} & -1 & $a^{\prime}$ & \begin{tabular}{|l|} 
\\
\end{tabular} & | & -1 & -1 & 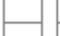 & 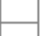 & -1 & $\square$ & $\square$ & -1 & & $\square$ & 足 & $\square$ \\
\hline & $\begin{array}{l}0-5 \% \\
0 \%\end{array}$ & - & - & $\rightarrow$ & $\square$ & $\square$ & & & 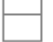 & $\square$ & & & + & & $\rightarrow$ & & $\rightarrow$ & 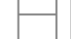 & & 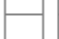 & $\rightarrow$ & 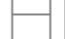 & - & 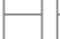 & $\square$ & -1 & & & 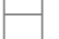 & & 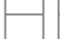 & & $\mapsto$ \\
\hline & Total & 100 & 100 & 100 & 100 & 100 & 100 & 100 & 100 & 100 & 100 & 100 & 100 & 100 & 100 & 100 & 100 & 100 & 100 & 100 & 100 & 100 & 100 & 100 & 100 & 100 & 100 & 100 & 100 & 100 & 100 & 100 & 100 \\
\hline & & & & & & & & efolia & ation & $=$ & LOW & & & & & & & & & & & & & efolia & atio & $\mathrm{el}=$ & $\mathrm{HIGH}$ & & & & & & $=-$ \\
\hline Starting In & asion State & Do & ninar & $\mathrm{nt}=\mathrm{s}$ & & & minan & $\mathrm{t}=\mathrm{C}$ & & Dor & minan & t $=\mathrm{k}$ & & Dor & $\operatorname{minan}$ & $\mathrm{t}=\mathrm{R}$ & & Dor & minar & $\mathrm{nt}=\mathrm{s}$ & & & $\operatorname{minan}$ & $\mathrm{nt}=\mathrm{C}$ & & & minan & $\mathrm{nt}=\mathrm{K}$ & & & $\operatorname{minan}$ & $t=F$ & \\
\hline & Ending State & $\mathrm{R}$ & G & $B$ & $D$ & $R$ & G & $\mathrm{B}$ & D & $R$ & G & B & D & $R$ & G & B & D & $R$ & G & B & D & $\mathrm{R}$ & G & B & D & $R$ & G & B & D & $R$ & G & B & D \\
\hline & SB & 80 & 50 & \begin{tabular}{|l|}
50 \\
\end{tabular} & \begin{tabular}{|l|}
50 \\
\end{tabular} & 25 & 15 & 15 & 15 & 5 & 5 & 5 & 5 & 5 & 5 & 5 & 5 & 60 & \begin{tabular}{|l|}
60 \\
\end{tabular} & \begin{tabular}{|l|l|}
600 \\
\end{tabular} & \begin{tabular}{|l|l|}
60 \\
\end{tabular} & 20 & 20 & 20 & 20 & 10 & 10 & 10 & 10 & 10 & 10 & 10 & 10 \\
\hline & $\mathrm{co}$ & 10 & 40 & 40 & 40 & 25 & 55 [ & 55 [ & 55 & 10 & 40 & 40 & 40 & 10 & 40 & 40 & 40 & 20 & 20 & 20 & 20 & 40 & 40[ & 40 [ & 40 & 20 & 20 & 20 & 20 & 20 & 20 & \begin{tabular}{|l|l|}
20 \\
\end{tabular} & 20 \\
\hline & KB & 5 & 5 & 5 & 5 & 25 & 15 & 15 & 15 & \begin{tabular}{|l|}
80 \\
\end{tabular} & \begin{tabular}{|l|}
50 \\
\end{tabular} & 50 & \begin{tabular}{|l|}
50 \\
\end{tabular} & 5 & 5 & & 5 & 10 & 10 & 10 & 10 & 20 & 20 & \begin{tabular}{|l|}
20 \\
\end{tabular} & 20 & \begin{tabular}{|l|}
60 \\
\end{tabular} & \begin{tabular}{|l|}
60 \\
\end{tabular} & \begin{tabular}{|c|c|}
60 \\
\end{tabular} & \begin{tabular}{|l|}
60 \\
\end{tabular} & 10 & 10 & 10 & 10 \\
\hline & RM & 5 & 5 & 5 & 5 & 25 & 15 & 15 & 15 & & & & & \begin{tabular}{|l|}
80 \\
\end{tabular} & \begin{tabular}{|c|}
50 \\
\end{tabular} & \begin{tabular}{|c|c|}
50 \\
\end{tabular} & \begin{tabular}{|l|}
50 \\
\end{tabular} & 10 & 10 & 10 & 10 & 20 & 20 & $20 \mid$ & \begin{tabular}{|l|}
20 \\
\end{tabular} & 10 & 10 & 10 & 10 & \begin{tabular}{|l|l|}
60 \\
\end{tabular} & \begin{tabular}{|c|c|}
60 \\
\end{tabular} & \begin{tabular}{|l|l|}
60 & \\
\end{tabular} & 60 \\
\hline & Total & 100 & 100 & 100 & 100 & 100 & 100 & 100 & 100 & 100 & 100 & 100 & 100 & 100 & 100 & 100 & 100 & 100 & 100 & 100 & 100 & 100 & 100 & 100 & 100 & 100 & 100 & 100 & 100 & 100 & 100 & 100 & 100 \\
\hline
\end{tabular}


Appendix 4b3. Modification of the tallgrass Model 3 elicitation response to provide a response for tallgrass Model 2.

[The sheet is designed the same as described in appendix 4a1 for the mixed-grass model, with one exception; the management actions for tallgrass are rest $(R)$, graze within window $(G)$, burn within window $(B)$, and defoliate outside of window (D)]

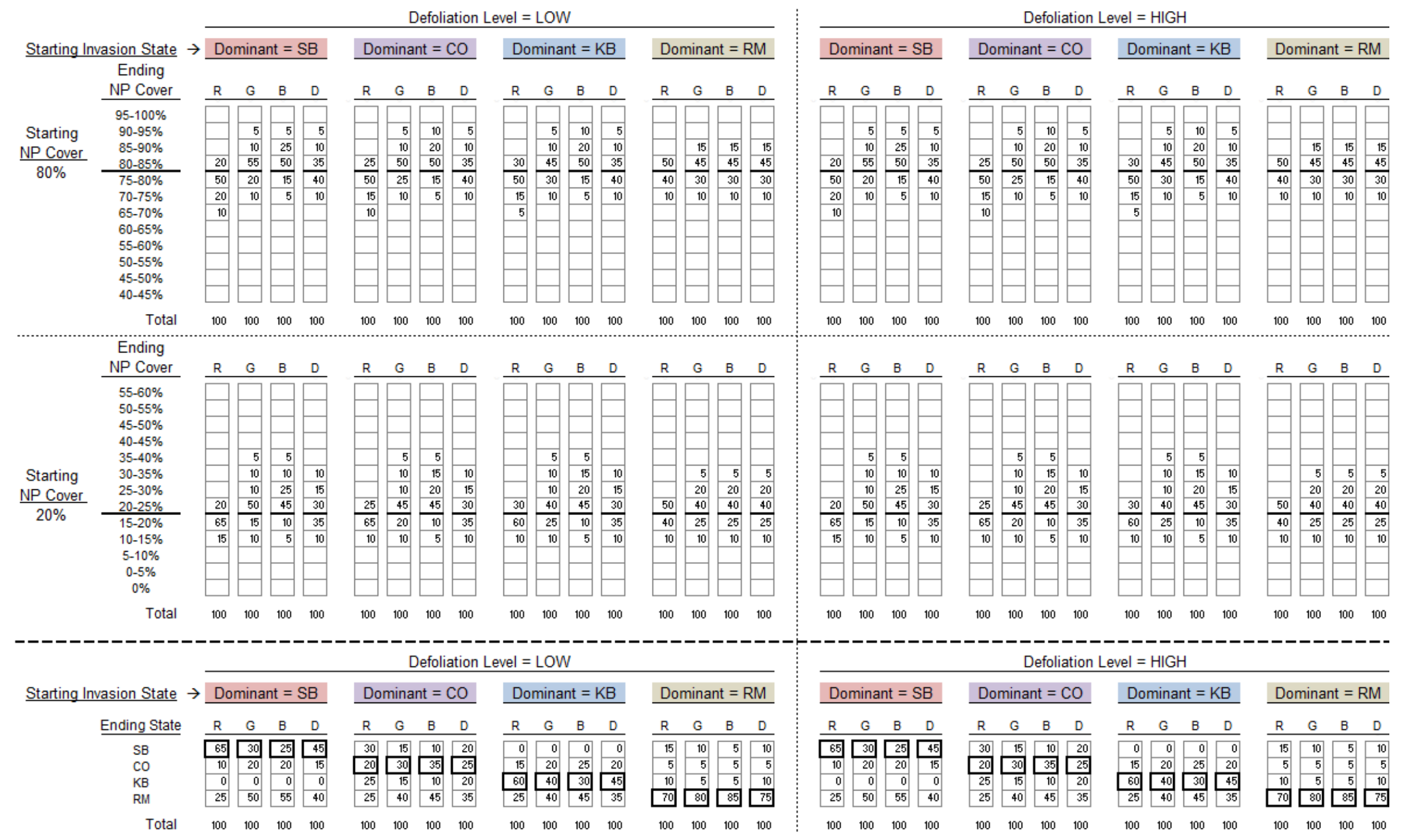


Appendix 4b4. Modification of the tallgrass Model 3 elicitation response to provide a response for tallgrass Model 4.

[The sheet is designed the same as described in appendix 4a1 for the mixed-grass model, with one exception; the management actions for tallgrass are rest $(R)$, graze within window $(G)$, burn within window $(B)$, and defoliate outside of window (D)]

\begin{tabular}{|c|c|c|c|c|c|c|c|c|c|c|c|c|c|c|c|c|c|c|c|c|c|c|c|c|c|c|c|c|c|c|c|c|c|}
\hline \multirow{3}{*}{\multicolumn{2}{|c|}{ 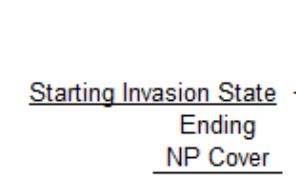 }} & \multicolumn{16}{|c|}{ Defoliation Level $=$ LOW } & \multicolumn{16}{|c|}{ Defoliation Level $=\mathrm{HIGH}$} \\
\hline & & \multicolumn{4}{|c|}{ Dominant $=\mathrm{SB}$} & \multicolumn{4}{|c|}{ Dominant $=\mathrm{CO}$} & \multicolumn{4}{|c|}{ Dominant $=\mathrm{KB}$} & \multicolumn{4}{|c|}{ Dominant $=\mathrm{RM}$} & \multicolumn{4}{|c|}{ Dominant $=\mathrm{SB}$} & \multicolumn{4}{|c|}{ Dominant $=\mathrm{CO}$} & \multicolumn{4}{|c|}{ Dominant $=\mathrm{KB}$} & \multicolumn{4}{|c|}{ Dominant = RM } \\
\hline & & $R$ & G & $\mathrm{B}$ & $D$ & $R$ & G & B & $D$ & $\mathrm{R}$ & G & B & D & $\mathrm{R}$ & G & B & D & $R$ & $G$ & $B$ & $D$ & $R$ & G & B & D & $\mathrm{R}$ & G & B & D & $\mathrm{R}$ & G & B & $\mathrm{D}$ \\
\hline \multirow{12}{*}{$\begin{array}{c}\text { Starting } \\
\text { NP Cover } \\
80 \%\end{array}$} & $95-100 \%$ & $\square$ & $\square$ & 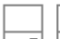 & $\square$ & $\square$ & $\square$ & \begin{tabular}{|l|} 
\\
\end{tabular} & -1 & $\square$ & $\square$ & $\square$ & $\square$ & $\square$ & \begin{tabular}{|l|l|} 
\\
\end{tabular} & $\square$ & 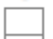 & $\square$ & $\square$ & $\square$ & $\square$ & $\square$ & $\square$ & $\square$ & $\square$ & $\square$ & $\square$ & & 4 & $\square$ & $\square$ & \begin{tabular}{|l|l|} 
\\
\end{tabular} & $\square$ \\
\hline & $90-95 \%$ & $\square$ & 5 & 5 & 5 & $\square$ & 5 & \begin{tabular}{|l|}
10 \\
\end{tabular} & 5 & $\square$ & \begin{tabular}{|l|}
5 \\
\end{tabular} & \begin{tabular}{|l|}
10 \\
\end{tabular} & 5 & $\square$ & \begin{tabular}{|l|} 
\\
\end{tabular} & \begin{tabular}{|l|} 
\\
\end{tabular} & & 5 & 5 & 5 & 5 & 5 & 5 & \begin{tabular}{|l|}
10 \\
\end{tabular} & 5 & 5 & 5 & 10 & 5 & & & & 4 \\
\hline & $85-90 \%$ & & 10 & \begin{tabular}{|l|}
25 \\
\end{tabular} & \begin{tabular}{|l|}
10 \\
\end{tabular} & 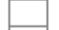 & 10 & \begin{tabular}{|l|}
20 \\
\end{tabular} & 10 & 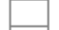 & 10 & \begin{tabular}{|l|}
20 \\
\end{tabular} & 10 & $\square$ & 15 & 15 & 15 & 20 & 10 & 25 & 10 & 20 & 10 & 20 & 10 & 25 & 10 & 20 & 10 & 20 & 15 & 15 & 15 \\
\hline & $80-85 \%$ & 20 & 55 & \begin{tabular}{|l|}
50 \\
\end{tabular} & 35 & 25 & 50 & \begin{tabular}{|l|}
50 \\
\end{tabular} & \begin{tabular}{|l|}
35 \\
\end{tabular} & 30 & 45 & \begin{tabular}{|l|}
50 \\
\end{tabular} & 35 & 50 & 45 & 45 & 45 & 55 & 55 & 50 & \begin{tabular}{|l|}
35 \\
\end{tabular} & 60 & 50 & \begin{tabular}{|l|}
50 \\
\end{tabular} & 35 & 60 & 45 & \begin{tabular}{|l|}
50 \\
\end{tabular} & 35 & 55 & 45 & \begin{tabular}{|l|}
45 \\
\end{tabular} & 45 \\
\hline & $75-80 \%$ & 50 & 20 & $\begin{array}{ll}15 \\
\end{array}$ & \begin{tabular}{|l|}
40 \\
\end{tabular} & 50 & 25 & \begin{tabular}{|l|}
15 \\
\end{tabular} & \begin{tabular}{|l|}
40 \\
\end{tabular} & 50 & \begin{tabular}{|l|}
30 \\
\end{tabular} & \begin{tabular}{|l|}
15 \\
\end{tabular} & 40 & \begin{tabular}{|l|}
40 \\
\end{tabular} & 30 & \begin{tabular}{|l|}
30 \\
\end{tabular} & \begin{tabular}{|l|}
30 \\
\end{tabular} & 15 & 20 & 15 & \begin{tabular}{|l|}
40 \\
\end{tabular} & 10 & \begin{tabular}{|l|}
25 \\
\end{tabular} & \begin{tabular}{|l|}
15 \\
\end{tabular} & 40 & 5 & \begin{tabular}{|l|}
30 \\
\end{tabular} & \begin{tabular}{|l|l|}
15 \\
\end{tabular} & 40 & 20 & \begin{tabular}{|l|}
30 \\
\end{tabular} & \begin{tabular}{|l|}
30 \\
\end{tabular} & 30 \\
\hline & $70-75 \%$ & 20 & 10 & 5 & 10 & 15 & 10 & 5 & \begin{tabular}{|l|}
10 \\
\end{tabular} & 15 & \begin{tabular}{|l|}
10 \\
\end{tabular} & 5 & 10 & 10 & 10 & 10 & 10 & 5 & 10 & 5 & $10 \mid$ & 5 & 10 & 5 & 10 & 5 & 10 & 5 & 10 & 5 & 10 & 10 & 10 \\
\hline & $65-70 \%$ & 10 & & - & & 10 & & $\square$ & \begin{tabular}{|l} 
\\
\end{tabular} & 5 & \begin{tabular}{|l|} 
\\
\end{tabular} & $\theta$ & $\square$ & $\square$ & \begin{tabular}{|l|l|l|l|} 
\\
\end{tabular} & $\square$ & \begin{tabular}{|l|} 
\\
\end{tabular} & $\square$ & $\square$ & $\square$ & $\square$ & $\square$ & $\square$ & \begin{tabular}{|l|} 
\\
\end{tabular} & $\square$ & $\square$ & $\square$ & \begin{tabular}{|l|} 
\\
\end{tabular} & $\square$ & $\square$ & $\square$ & $\square$ & $\square$ \\
\hline & $\begin{array}{l}60-65 \% \\
55-600 \%\end{array}$ & $\square$ & $\square$ & $\square$ & $\square$ & $\square$ & 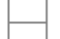 & $\square$ & $\square$ & $\square$ & $\square$ & $\left.\right|^{-1}$ & 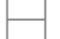 & $\square$ & $\square$ & $\square$ & 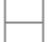 & $\square$ & - & -1 & 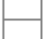 & $\square$ & $\square$ & $\square$ & $\square$ & - & 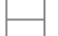 & 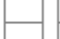 & 足 & $\square$ & 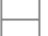 & \begin{tabular}{|l} 
\\
\end{tabular} & 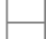 \\
\hline & $\begin{array}{l}55-60 \% \\
50-55 \%\end{array}$ & 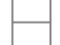 & -1 & 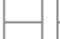 & 直 & $\mid$ & | & $\left.\right|^{-1}$ & \begin{tabular}{|l} 
\\
\end{tabular} & + & $\mapsto$ & 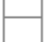 & $\square$ & $\square$ & 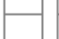 & 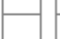 & 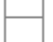 & $\square$ & $\square$ & $\theta$ & $\rightarrow$ & $\square$ & $\square$ & $\longrightarrow$ & $\square$ & - & $\square$ & $\mapsto$ & $\rightarrow$ & - & $\rightarrow$ & $一$ & $\mapsto$ \\
\hline & $45-50 \%$ & $\square$ & - & $\square$ & $\rightarrow$ & $\square$ & $\square$ & $\square$ & $\square$ & $\square$ & $\square$ & $\square$ & $\square$ & $\square$ & $\square$ & \begin{tabular}{|l|} 
\\
\end{tabular} & $\square$ & $\square$ & $\square$ & $\square$ & $\square$ & $\square$ & 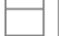 & $\square$ & $\rightarrow$ & - & 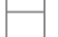 & $\square$ & -1 & 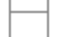 & $\square$ & $\square$ & $\square$ \\
\hline & $40-45 \%$ & $\square$ & $\square$ & \begin{tabular}{|l} 
\\
\end{tabular} & \begin{tabular}{|l} 
\\
\end{tabular} & $\square$ & \begin{tabular}{|l} 
\\
\end{tabular} & \begin{tabular}{|l} 
\\
\end{tabular} & $\square$ & $\square$ & \begin{tabular}{|l|l} 
\\
\end{tabular} & $\square$ & $\square$ & $\square$ & $\square[$ & $\square[$ & $\square$ & $\square$ & \begin{tabular}{|l} 
\\
\end{tabular} & $\square$ & $\square$ & $\square$ & \begin{tabular}{|l|} 
\\
\end{tabular} & \begin{tabular}{|l} 
\\
\end{tabular} & $\square$ & $\square$ & \begin{tabular}{|l} 
\\
\end{tabular} & \begin{tabular}{|l|} 
\\
\end{tabular} & \begin{tabular}{|l}
$\square$ \\
\end{tabular} & $\square$ & \begin{tabular}{|l|} 
\\
\end{tabular} & \begin{tabular}{|l} 
\\
\end{tabular} & 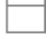 \\
\hline & Total & 100 & 100 & 100 & 100 & 100 & 100 & 100 & 100 & 100 & 100 & 100 & 100 & 100 & 100 & 100 & 100 & 100 & 100 & 100 & 100 & 100 & 100 & 100 & 100 & 100 & 100 & 100 & 100 & 100 & 100 & 100 & 100 \\
\hline \multirow{15}{*}{$\begin{array}{c}\text { Starting } \\
\text { NP Cover } \\
20 \%\end{array}$} & Ending & & & & & & & & & & & & & & & & & & & & & & & & & & & & & & & & \\
\hline & NP Cover & $R$ & G & B & D & $R$ & G & B & D & $\mathrm{R}$ & G & $B$ & $\mathrm{D}$ & $\mathrm{R}$ & G & $B$ & $\mathrm{D}$ & $R$ & $G$ & $B$ & $D$ & $\mathrm{R}$ & G & $B$ & $D$ & $\mathrm{R}$ & G & $\mathrm{B}$ & D & $R$ & $G$ & $B$ & $\mathrm{D}$ \\
\hline & $55-60 \%$ & 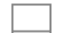 & \begin{tabular}{|l|} 
\\
\end{tabular} & $\square$ & 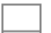 & $\square$ & 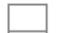 & \begin{tabular}{|l|} 
\\
\end{tabular} & $\square$ & $\square$ & $\square$ & $\square$ & $\square$ & $\square$ & $\square$ & 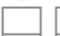 & $\square$ & $\square$ & $\square$ & 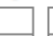 & $\square$ & $\square$ & $\square$ & - & 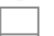 & $\square$ & - & 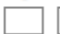 & $\square$ & & $\square$ & - & $\square$ \\
\hline & $50-55 \%$ & 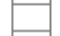 & \begin{tabular}{|l} 
\\
\end{tabular} & $\square$ & a & $\square$ & $\square$ & \begin{tabular}{|l|} 
\\
\end{tabular} & $\square$ & 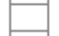 & \begin{tabular}{|l|} 
\\
\end{tabular} & $\square$ & $\square$ & $\square$ & \begin{tabular}{|l|} 
\\
\end{tabular} & \begin{tabular}{|l|} 
\\
\end{tabular} & $\square$ & & $\square$ & 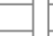 & \begin{tabular}{|l|} 
\\
\end{tabular} & $\square$ & \begin{tabular}{|l|} 
\\
\end{tabular} & \begin{tabular}{|l|} 
\\
\end{tabular} & $\square$ & 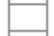 & 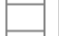 & | & \begin{tabular}{|l} 
\\
\end{tabular} & 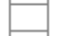 & 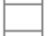 & 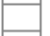 & \begin{tabular}{|l|} 
\\
\end{tabular} \\
\hline & $45-50 \%$ & 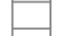 & $\square$ & $\square$ & $\square$ & $\square$ & \begin{tabular}{|l|} 
\\
\end{tabular} & \begin{tabular}{|l|} 
\\
\end{tabular} & \begin{tabular}{|l|} 
\\
\end{tabular} & $\square$ & \begin{tabular}{|l|} 
\\
\end{tabular} & \begin{tabular}{|l|} 
\\
\end{tabular} & $\square$ & $\square$ & \begin{tabular}{|l} 
\\
\end{tabular} & \begin{tabular}{|l|} 
\\
\end{tabular} & $\square$ & $\square$ & $\square$ & $\square$ & $\square$ & $\square$ & \begin{tabular}{|l|} 
\\
\end{tabular} & $\square$ & $\square$ & $\square$ & \begin{tabular}{|l} 
\\
\end{tabular} & \begin{tabular}{|l|} 
\\
\end{tabular} & $\square$ & $\square$ & - & \begin{tabular}{|l} 
\\
\end{tabular} & $\square$ \\
\hline & $4 C$ & 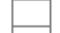 & $\square$ & $\square$ & $\square$ & $\square$ & $\square$ & \begin{tabular}{|l|} 
\\
\end{tabular} & $\square$ & $\square$ & $\square$ & $\square$ & $\square$ & $\square$ & \begin{tabular}{|l|} 
\\
\end{tabular} & $\square$ & $\square$ & $\square$ & $\square$ & $\square$ & $\square$ & $\square$ & $\square$ & $\square$ & $\square$ & $\square$ & $\square$ & \begin{tabular}{|l|} 
\\
\end{tabular} & $\square$ & $\square$ & - & \begin{tabular}{|l} 
\\
\end{tabular} & $\square$ \\
\hline & $35-40 \%$ & - & - & \begin{tabular}{|l} 
\\
\end{tabular} & $\square$ & $\square$ & -1 & \begin{tabular}{|l} 
\\
\end{tabular} & - & 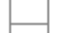 & 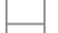 & \begin{tabular}{|l|l|} 
\\
\end{tabular} & $\square$ & - & \begin{tabular}{|l} 
\\
\end{tabular} & \begin{tabular}{|l} 
\\
\end{tabular} & $\square$ & $=$ & & $\square$ & - & 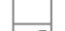 & \begin{tabular}{|l} 
\\
\end{tabular} & 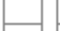 & & & & \begin{tabular}{|l} 
\\
\end{tabular} & & 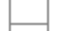 & \begin{tabular}{|l} 
\\
\end{tabular} & \begin{tabular}{|l} 
\\
\end{tabular} & $\square$ \\
\hline & $35 \%$ & - & - & \begin{tabular}{|l} 
\\
\end{tabular} & \begin{tabular}{|l} 
\\
\end{tabular} & - & - & \begin{tabular}{|l} 
\\
\end{tabular} & \begin{tabular}{|l} 
\\
\end{tabular} & $\square$ & \begin{tabular}{|l} 
\\
\end{tabular} & -1 & 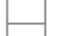 & 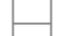 & \begin{tabular}{|l} 
\\
\end{tabular} & \begin{tabular}{|l} 
\\
\end{tabular} & $\square$ & 5 & 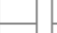 & - & 品 & 5 & - & \begin{tabular}{|l} 
\\
\end{tabular} & \begin{tabular}{|l} 
\\
\end{tabular} & 5 & \begin{tabular}{|l} 
\\
\end{tabular} & \begin{tabular}{|l} 
\\
\end{tabular} & - & 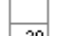 & \begin{tabular}{|l} 
\\
\end{tabular} & \begin{tabular}{|l} 
\\
\end{tabular} & $\square$ \\
\hline & $25-30 \%$ & 20 & 20 & \begin{tabular}{|l|} 
\\
20
\end{tabular} & 20 & 25 & 25 & \begin{tabular}{|l|}
25 \\
25
\end{tabular} & & . & 3 & \begin{tabular}{|l}
30 \\
\end{tabular} & 30 & 5 & $=$ & $|5|$ & \begin{tabular}{|l|}
50 \\
\end{tabular} & 20 & & 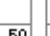 & & 20 & & 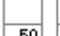 & & 25 & 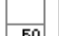 & 50 & $\square$ & \begin{tabular}{|l|}
20 \\
55
\end{tabular} & 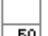 & \begin{tabular}{|l|l|} 
\\
50
\end{tabular} & $\square$ \\
\hline & $\frac{20-25 \%}{15-20 \%}$ & $\frac{20}{65}$ & \begin{tabular}{|l|}
20 \\
65
\end{tabular} & \begin{tabular}{|l|}
20 \\
65
\end{tabular} & $\frac{20}{65}$ & $\begin{array}{l}25 \\
65\end{array}$ & \begin{tabular}{|l|}
25 \\
65
\end{tabular} & $\frac{25}{65}$ & $\frac{25}{65}$ & $\frac{30}{60}$ & $\frac{30}{60}$ & $\frac{30}{60}$ & $\frac{30}{60}$ & $\frac{50}{40}$ & \begin{tabular}{|l|}
50 \\
40
\end{tabular} & $\frac{50}{40}$ & $\frac{50}{40}$ & $\begin{array}{r}55 \\
15\end{array}$ & $\frac{50}{40}$ & $\frac{50}{40}$ & $\frac{50}{40}$ & $\frac{60}{10}$ & \begin{tabular}{|l|}
50 \\
40
\end{tabular} & \begin{tabular}{|l|}
50 \\
40
\end{tabular} & $\frac{50}{40}$ & $\frac{60}{5}$ & $\frac{50}{40}$ & $\frac{50}{440}$ & \begin{tabular}{|l}
50 \\
40
\end{tabular} & $\begin{array}{l}55 \\
20\end{array}$ & $\frac{50}{40}$ & $\frac{50}{40}$ & \begin{tabular}{|l|}
50 \\
40
\end{tabular} \\
\hline & $10-15 \%$ & 15 & 15 & \begin{tabular}{|c|}
15 \\
\end{tabular} & \begin{tabular}{|l|}
15 \\
\end{tabular} & 10 & \begin{tabular}{|c|}
10 \\
\end{tabular} & \begin{tabular}{|c|}
10 \\
\end{tabular} & \begin{tabular}{|c|}
10 \\
\end{tabular} & 10 & \begin{tabular}{|c|}
10 \\
\end{tabular} & \begin{tabular}{|c|}
10 \\
\end{tabular} & \begin{tabular}{|c|}
10 \\
\end{tabular} & \begin{tabular}{|c|}
10 \\
\end{tabular} & \begin{tabular}{|c|}
10 \\
\end{tabular} & \begin{tabular}{|c|}
10 \\
\end{tabular} & 10 & 5 & 10 & 10 & \begin{tabular}{|c|}
10 \\
\end{tabular} & 5 & \begin{tabular}{|c|}
10 \\
\end{tabular} & \begin{tabular}{|c|}
10 \\
\end{tabular} & \begin{tabular}{|l|}
10 \\
\end{tabular} & 5 & \begin{tabular}{|c|}
10 \\
\end{tabular} & \begin{tabular}{|c|}
10 \\
\end{tabular} & 10 & \begin{tabular}{|r|}
5 \\
\end{tabular} & \begin{tabular}{|l|}
10 \\
\end{tabular} & 10 & \begin{tabular}{|c|}
10 \\
\end{tabular} \\
\hline & $5-10 \%$ & - & & & 7 & $\square$ & & $\square$ & & $\square$ & \begin{tabular}{|l} 
\\
\end{tabular} & $\square$ & $\square$ & $\square$ & \begin{tabular}{|l|} 
\\
\end{tabular} & $\square$ & $\square$ & 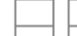 & $\square$ & $\square$ & $\square$ & $\square$ & $\square$ & \begin{tabular}{|l} 
\\
\end{tabular} & $\square$ & & \begin{tabular}{|l|} 
\\
\end{tabular} & \begin{tabular}{|l|} 
\\
\end{tabular} & $\square$ & $\square$ & \begin{tabular}{|l} 
\\
\end{tabular} & $\square$ & $\square$ \\
\hline & $0-5 \%$ & L & -1 & \begin{tabular}{|l|l} 
\\
\end{tabular} & | & - & -1 & \begin{tabular}{|l} 
\\
\end{tabular} & - & 表 & 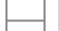 & \begin{tabular}{|l|} 
\\
\end{tabular} & 员 & 员 & \begin{tabular}{|l|l} 
\\
\end{tabular} & & | & — & & & - & H & & & & - & & & & 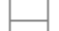 & & & \\
\hline & $0 \%$ & $\square$ & $\square$ & $\square$ & $\square$ & $\square$ & $\square$ & $\square$ & $\square$ & $\square$ & $\square$ & $\square$ & $\square$ & $\square$ & $\square$ & $\square$ & $\square$ & 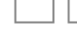 & $\square$ & 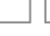 & $\square$ & $\square$ & $\square$ & $\square$ & $\square$ & 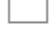 & 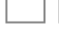 & $\square$ & $\square$ & $\square$ & $\square$ & $\square$ & $\square$ \\
\hline & Total & 100 & 100 & 100 & 100 & 100 & 100 & 100 & 100 & 100 & 100 & 100 & 100 & 100 & 100 & 100 & 100 & 100 & 100 & 100 & 100 & 100 & 100 & 100 & 100 & 100 & 100 & 100 & 100 & 100 & 100 & 100 & 100 \\
\hline & & & & & & & & efolia & ation & $\mathrm{el}=$ & LOW & & & & & & & & & & & & & efolia & ation & ivel $=$ & $\mathrm{HIGH}$ & & & & & & \\
\hline Starting In & vasion State & Do & minar & $\mathrm{nt}=\mathrm{s}$ & & Dor & minar & $\mathrm{t}=\mathrm{c}$ & & Do & minan & $\mathrm{nt}=\mathrm{r}$ & & Dor & minan & t $=R$ & & Dom & ninant & $t=s$ & & Dor & $\operatorname{minan}$ & $\mathrm{nt}=\mathrm{c}$ & & & minan & $\mathrm{nt}=\mathrm{K}$ & & Dor & mina & $\mathrm{nt}=\mathrm{F}$ & $\mathrm{RM}$ \\
\hline & Ending State & $\mathrm{R}$ & G & $B$ & D & $\mathrm{R}$ & $G$ & $B$ & $D$ & $\mathrm{R}$ & G & B & D & $\mathrm{R}$ & G & B & D & $R$ & G & B & D & $R$ & G & $B$ & D & $R$ & G & $B$ & D & $R$ & G & $B$ & D \\
\hline & SB & 85 & 65 & 60 & 70 & 40 & 30 & 30 & 35 & 0 & 0 & 0 & 0 & 35 & \begin{tabular}{|l|l|}
25 \\
\end{tabular} & \begin{tabular}{|l|l|}
25 \\
\end{tabular} & 30 & 35 & 35 [ & 35 [ & 35 & 10 & 10 & \begin{tabular}{|l|}
10 \\
\end{tabular} & 10 & 0 & 0 & 0 & 0 & 10 & 10 & 10 & 10 \\
\hline & $\mathrm{co}$ & 10 & 25 & \begin{tabular}{|l|}
25 \\
\end{tabular} & 20 & 20 & \begin{tabular}{|l|}
30 \\
\end{tabular} & 35 [ & \begin{tabular}{|l|}
30 \\
\end{tabular} & 20 & 30 & 35 & 25 & \begin{tabular}{|l|l|}
20 \\
\end{tabular} & \begin{tabular}{|l|l|}
30 \\
\end{tabular} & \begin{tabular}{|l|}
30 \\
\end{tabular} & 25 & 55 & 55 & 55 & 55 & 75 & \begin{tabular}{|l|l|}
75 \\
\end{tabular} & \begin{tabular}{|l|}
75 \\
\end{tabular} & 75 & 65 & \begin{tabular}{|l|}
65 \\
6
\end{tabular} & 65 & 65 & 50 & \begin{tabular}{|l|}
50 \\
\end{tabular} & 50 & 50 \\
\hline & KB & 0 & 0 & 0 & 0 & 35 & 30 & 25 & \begin{tabular}{|l|}
30 \\
\end{tabular} & \begin{tabular}{|l|l|}
75 \\
\end{tabular} & \begin{tabular}{|l|}
65 \\
\end{tabular} & \begin{tabular}{|l|}
55 \\
\end{tabular} & \begin{tabular}{|l|l|}
70 \\
\end{tabular} & \begin{tabular}{|l|}
30 \\
\end{tabular} & 25 & 20 & 25 & 0 & 0 & 0 & 0 & 5 & 5 & 5 & 5 & \begin{tabular}{|l|}
25 \\
\end{tabular} & \begin{tabular}{|l|}
25 \\
\end{tabular} & \begin{tabular}{|l|}
25 \\
\end{tabular} & \begin{tabular}{|l|}
25 \\
\end{tabular} & 5 & 5 & 5 & 5 \\
\hline & RM & 5 & 10 & 15 & 10 & 5 & 10 & \begin{tabular}{|l|}
10 \\
\end{tabular} & 5 & 5 & 5 & \begin{tabular}{|l|}
10 \\
\end{tabular} & 5 & \begin{tabular}{|l|}
15 \\
\end{tabular} & \begin{tabular}{|l|}
20 \\
\end{tabular} & 25[ & \begin{tabular}{|l|}
20 \\
\end{tabular} & 10 & \begin{tabular}{|l|l|}
10 \\
\end{tabular} & 10 & 10 & 10 & 10 & \begin{tabular}{|l|}
10 \\
\end{tabular} & 10 & 10 & \begin{tabular}{|c|}
10 \\
\end{tabular} & \begin{tabular}{|l|}
10 \\
\end{tabular} & 10 & \begin{tabular}{|l|l|}
35 \\
\end{tabular} & \begin{tabular}{|l|}
35 \\
\end{tabular} & \begin{tabular}{|l|}
35 \\
\end{tabular} & \begin{tabular}{|l|}
35 \\
\end{tabular} \\
\hline & Total & 100 & 100 & 100 & 100 & 100 & 100 & 100 & 100 & 100 & 100 & 100 & 100 & 100 & 100 & 100 & 100 & 100 & 100 & 100 & 100 & 100 & 100 & 100 & 100 & 100 & 100 & 100 & 100 & 100 & 100 & 100 & 100 \\
\hline
\end{tabular}


Appendix 4b5. Modification of the tallgrass Model 3 elicitation response to provide a response for tallgrass Model 5.

[The sheet is designed the same as described in appendix 4a1 for the mixed-grass model, with one exception; the management actions for tallgrass are rest $(R)$, graze within window $(G)$, burn within window $(B)$, and defoliate outside of window (D)]

Defoliation Level $=$ LOW

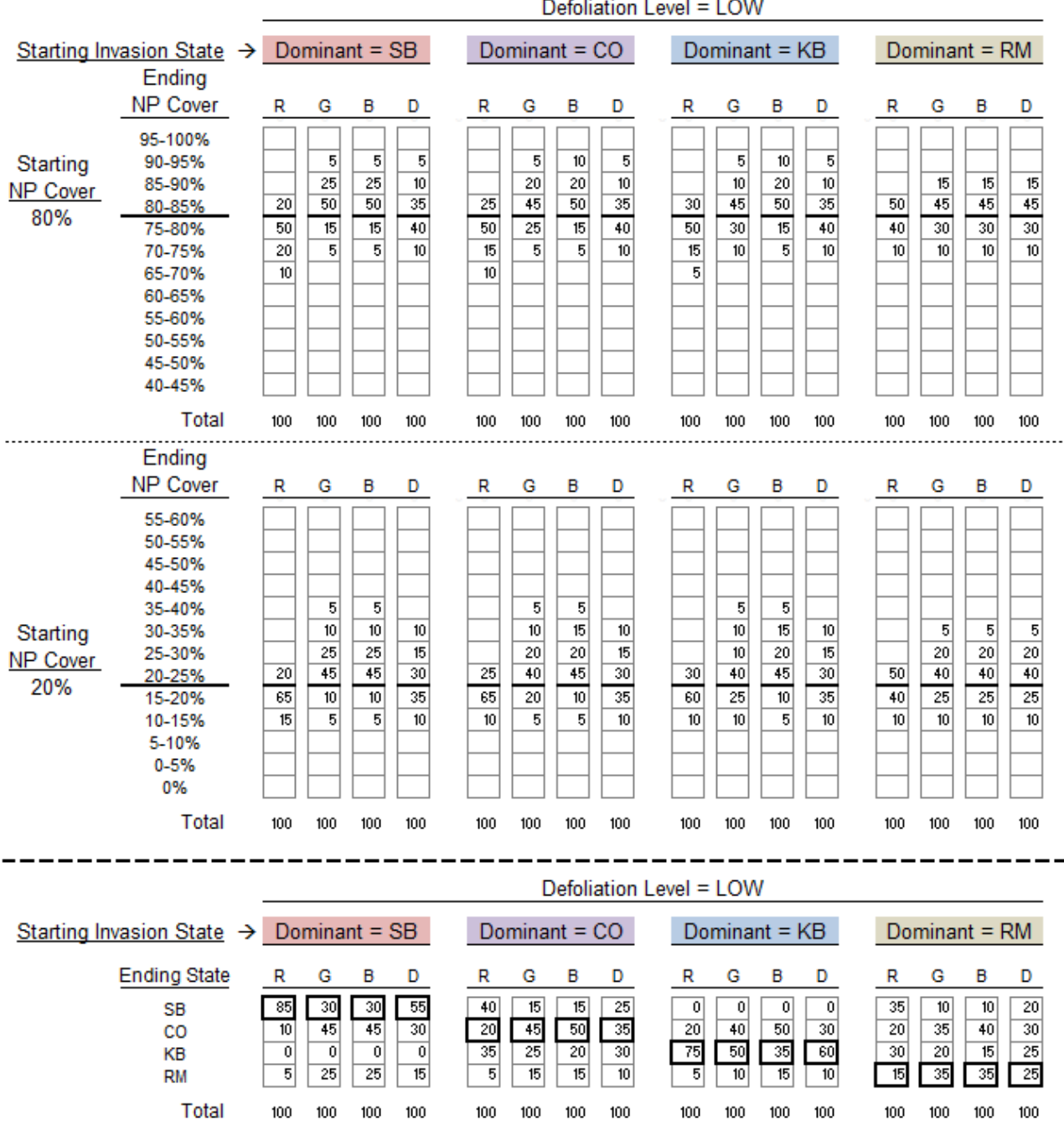

Defoliation Level $=\mathrm{HIGH}$

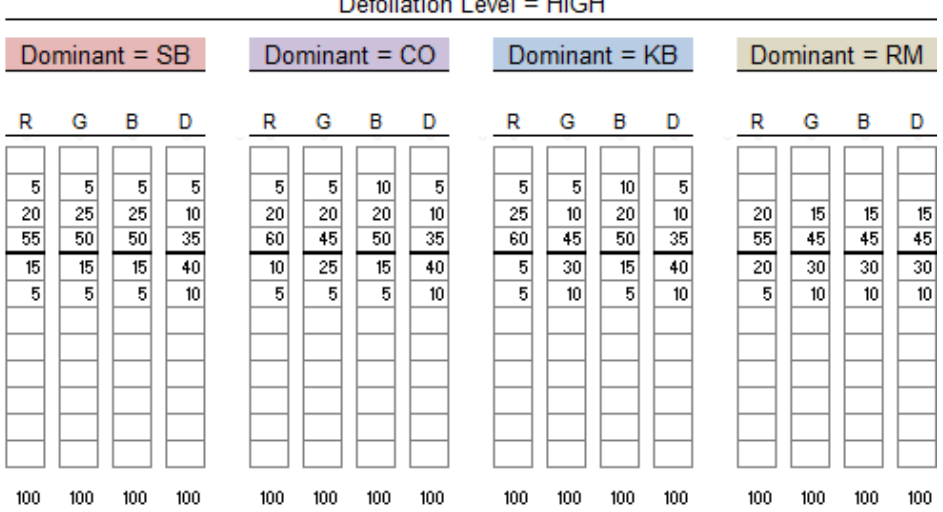

$100 \quad 100 \quad 100 \quad 100$

$100 \quad 100 \quad 100$

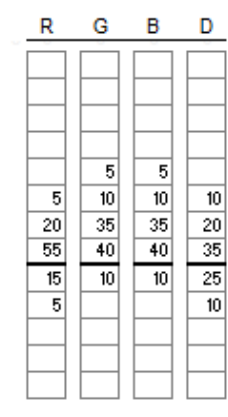

$100 \quad 100 \quad 100 \quad 100$

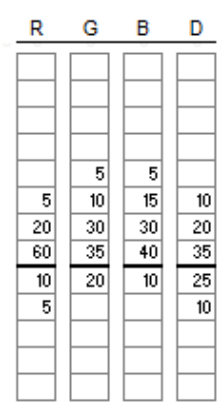

$100 \quad 100 \quad 100 \quad 100$

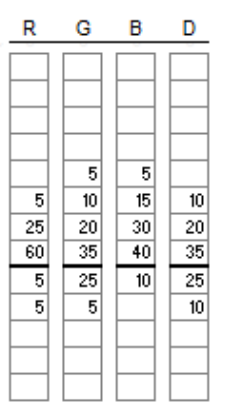

$100 \quad 100 \quad 100 \quad 100$

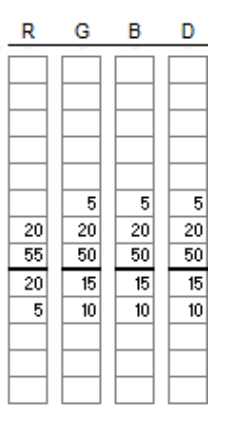

$100 \quad 100 \quad 100 \quad 100$

\section{Defoliation Level $=\mathrm{HIGH}$}

Dominant $=\mathrm{SB} \quad$ Dominant $=\mathrm{CO} \quad$ Dominant $=\mathrm{KB} \quad$ Dominant $=\mathrm{RM}$

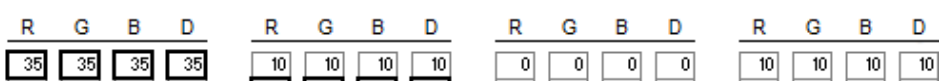

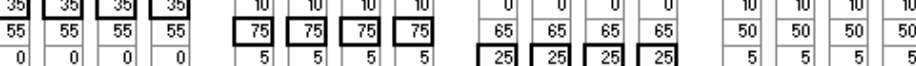

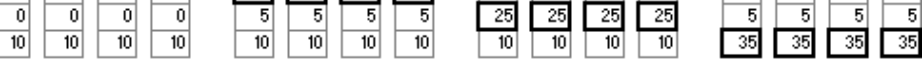
$\begin{array}{llllllllllllllll}100 & 100 & 100 & 100 & 100 & 100 & 100 & 100 & 100 & 100 & 100 & 100 & 100 & 100 & 100 & 100\end{array}$ 
Appendix 4b6. Modification of the tallgrass Model 3 elicitation response to provide a response for tallgrass Model 6 .

[The sheet is designed the same as described in appendix 4a1 for the mixed-grass model, with one exception; the management actions for tallgrass are rest (R), graze within window (G), burn within window (B), and defoliate outside of window (D)]

Defoliation Level $=$ LOW

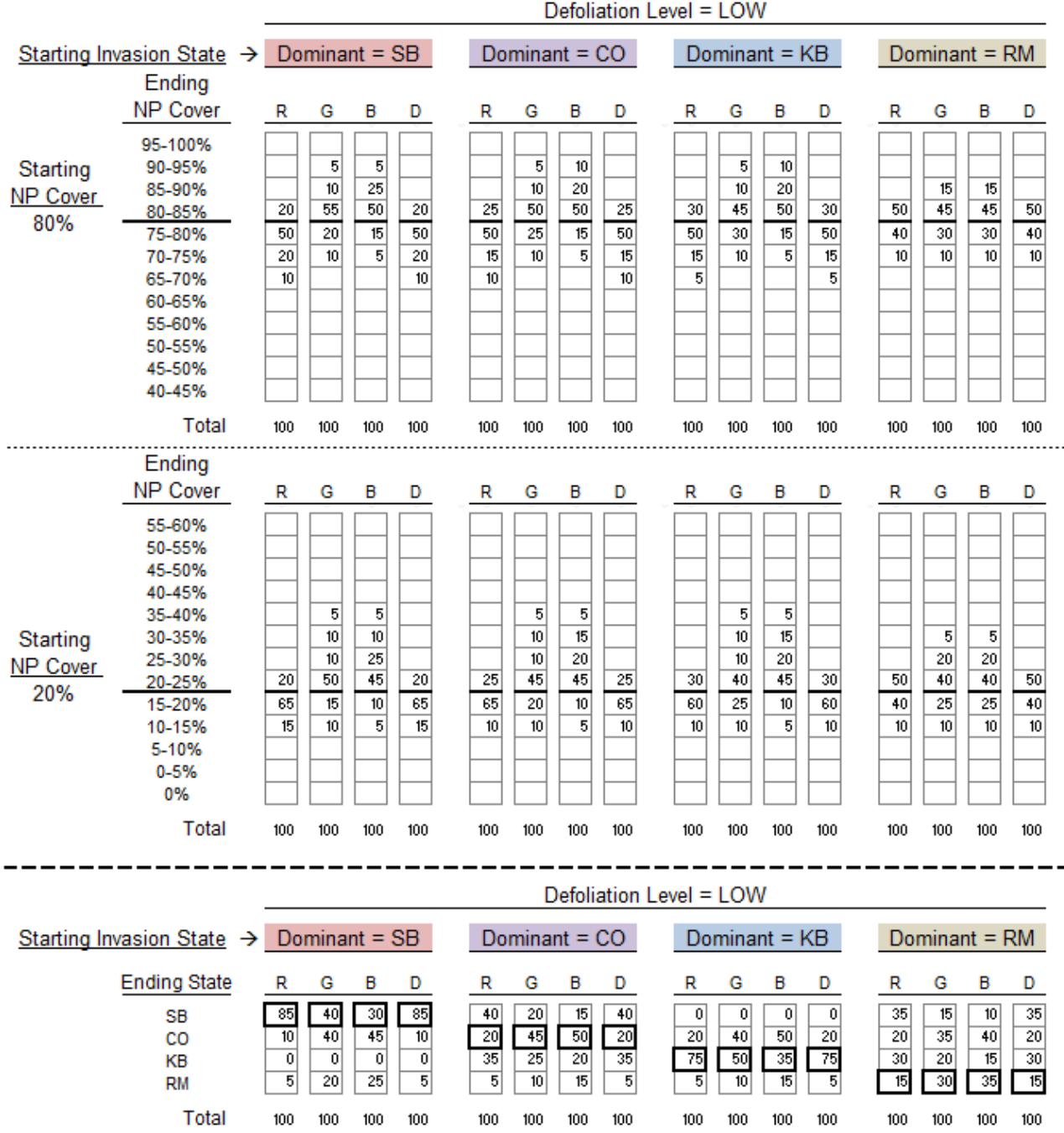

Defoliation Level $=\mathrm{HIGH}$

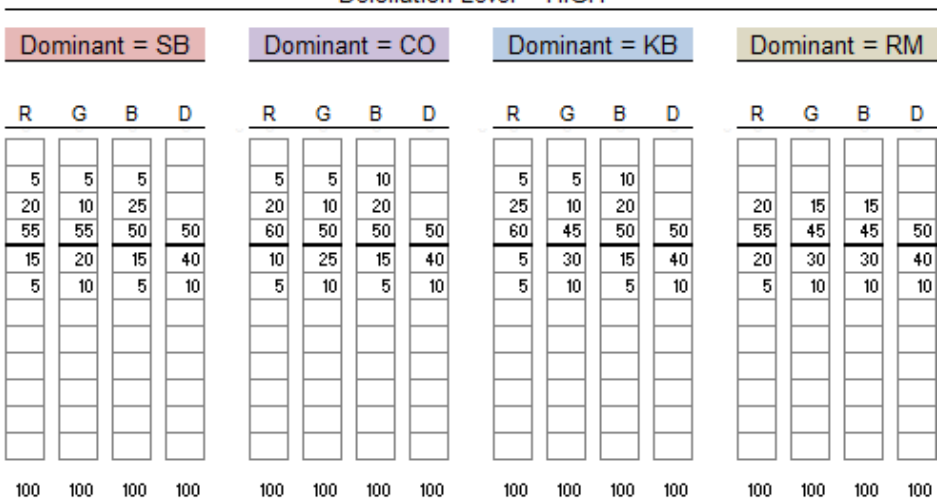

$\begin{array}{llll}100 & 100 & 100 & \end{array}$

$100 \quad 100 \quad 100 \quad 100$
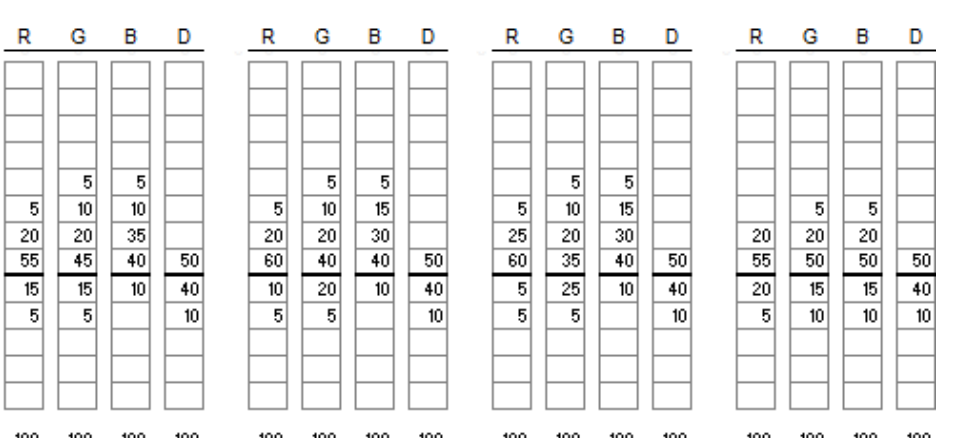

$100 \quad 100 \quad 100 \quad 100$

$100 \quad 100 \quad 100 \quad 100$

$100 \quad 100 \quad 100 \quad 100$

$100 \quad 100 \quad 100 \quad 100$

Defoliation Level $=\mathrm{HIGH}$

Dominant $=\mathrm{SB}$ Dominant $=\mathrm{CO}$ Dominant $=\mathrm{KB}$ Dominant $=\mathrm{RM}$

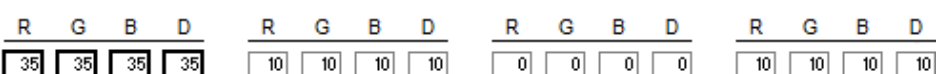

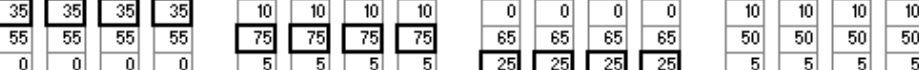

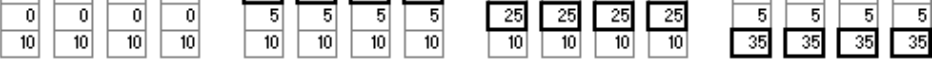

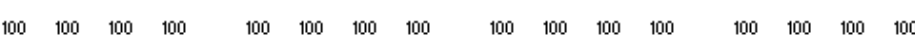


Appendix 5a. Modifications to the mixed-grass baseline model elicitation spreadsheet to derive values for the alternative models.

[Modifications are targeted at the distinctive aspects of each alternative model relative to the baseline model. Below, we summarize the conceptual model of all four models, starting with the baseline model, Model 3. Additionally, for Models 1, 2, and 4, we pinpoint the distinctive feature of each model compared with Model 3 and describe how the values of the baseline elicitation spreadsheet were modified to accurately represent the conceptual models of each alternative model. The elicitation spreadsheets for all mixed-grass models are located in appendixes $4 \mathrm{a} 1-4 \mathrm{a} 4 . \mathrm{SB}=$ smooth brome, $\mathrm{KB}=$ Kentucky bluegrass, $\mathrm{CO}=$ co-dominant smooth brome and Kentucky bluegrass, $\mathrm{RM}=$ remainder, $\mathrm{NP}=$ native prairie]

Model 3 (Baseline Model): Invader Type, Defoliation Level

Invader Type

- Management actions are differentially effective depending on the dominant invader, as follows:

- SB: Burn $/$ Graze $=$ Graze $>$ Burn $>$ Rest

- CO: Burn $/$ Graze $>$ Burn $=$ Graze $>$ Rest

- KB: Burn/Graze $=$ Burn $>$ Graze $>$ Rest

- RM: Burn $/$ Graze $=$ Burn $=$ Graze $>$ Rest

Defoliation Level

- Past management history affects the effect of current management actions. Actions have different effectiveness depending on whether the defoliation level is low or high, as follows:

$\circ$ Rest is less detrimental under a high defoliation level than under a low defoliation level.

- At a low native cover (that is, 20 percent NP), active management actions are more effective under a high defoliation level than under a low defoliation level.

Invasion Level

- Management action effectiveness is not density-dependent.

Model 1: Not state-based

\section{Upper section of spreadsheet for change in native cover} Invader Type

- Effectiveness of management actions is not invader-specific; all forms of disturbance are equivalent and better than doing nothing (that is, rest).

- SB: Burn $/$ Graze $=$ Burn $=$ Graze $>$ Rest

- CO: Burn $/$ Graze $=$ Burn $=$ Graze $>$ Rest

- KB: Burn $/$ Graze $=$ Burn $=$ Graze $>$ Rest

- RM: Burn $/$ Graze $=$ Burn $=$ Graze $>$ Rest

Modification to baseline model elicitation spreadsheet values

- Rest: We set the values for rest to the average of the rest values over all four dominance types ( $\mathrm{SB}, \mathrm{CO}, \mathrm{KB}, \mathrm{RM}$ ).

- We averaged across the native cover starting levels (that is, across 80 percent NP and 20 percent NP).

- For the low defoliation level, we averaged across the low values.

- For the high defoliation level, we averaged across the low and the high values.

- Burn/Graze, Burn, Graze: We set the values for burn/graze, burn, and graze equal to the average of the three actions over all four dominance types.

- For the low defoliation level, we averaged across native cover starting levels (that is, across 80 percent NP and 20 percent NP). 
Defoliation Level

- For the high defoliation level, see below.

- Past management history does not affect management effectiveness.

$>$ Modification to baseline model elicitation spreadsheet values

- Burn/Graze, Burn, Graze

- At high defoliation level, we set the values for burn/graze, burn, and graze equal to rest (at 80 percent NP and 20 percent NP).

Invasion Level

- At low defoliation level, see above.

- Management action effectiveness is not density-dependent.

$>$ Modification to baseline model elicitation spreadsheet values

○ No modification necessary.

\section{Lower section of spreadsheet for change in dominance}

$>$ Modification to baseline model elicitation spreadsheet values-Dominance section

- Using the dominance spreadsheet, we changed rest probabilities and management action effects according to the values in the upper section of the spreadsheet (digital appendix $7 \mathrm{a} 2)$.

- Modified so does not recognize a difference between SB, KB, or RM (because not statebased).

Model 2: Invader Type

\section{Upper section of spreadsheet for change in native cover}

Invader Type

- Management actions are differentially effective depending on the dominant invader.

$>$ Modification to baseline model elicitation spreadsheet values

○ No modification necessary.

Defoliation Level

- Past management history does not affect management effectiveness.

$>$ Modification to baseline model elicitation spreadsheet values

- At 80 percent NP, low and high defoliation level

- Rest: Set rest high values equal to rest low values for each dominance type.

- Burn/Graze, Burn, Graze: No change to burn/graze, burn, or graze; the low and high values for each of these actions are already equal at 80 percent NP.

$\circ$ At 20 percent NP, low and high defoliation level

- Rest: Set rest high values equal to rest low values for each dominance type.

- Burn/Graze, Burn, Graze: Set burn/graze, burn, and graze high values equal to their low values for each action at each dominance type.

Invasion Level

- Management action effectiveness is not density-dependent.

$>$ Modification to baseline model elicitation spreadsheet values

○ No modification necessary.

\section{Lower section of spreadsheet for change in dominance}

$>$ Modification to baseline model elicitation spreadsheet values-Dominance section 
- Using the dominance spreadsheet, we changed rest probabilities and management action effects according to the values in the upper section of the spreadsheet (digital appendix $7 \mathrm{a} 3)$.

Model 4: Invader Type, Defoliation Level, Invasion Level

\section{Upper section of spreadsheet for change in native cover}

Invader Type

- Management actions are differentially effective depending on the dominant invader.

$>$ Modification to baseline model elicitation spreadsheet values

○ No modification necessary.

Defoliation Level

- Past management history does affect management effectiveness.

$>$ Modification to baseline model elicitation spreadsheet values

○ No modification necessary.

Invasion Level

- Effectiveness of management action is density dependent.

$\circ$ Effectiveness declines as the level of invasion increases.

$\circ$ At 20 percent NP, active management actions are no more effective than rest.

$>$ Modification to baseline model elicitation spreadsheet values

- At 20 percent NP, low defoliation level

- Set burn/graze, burn, and graze equal to the rest values within each dominance type.

- At 20 percent NP, high defoliation level

- Set burn/graze, burn, and graze to a 50|50 split above and below the bar.

\section{Lower section of spreadsheet for change in dominance}

$>$ Modification to baseline model elicitation spreadsheet values-Dominance section

- Using the dominance spreadsheet, we changed management action effects according to the values in the upper section of the spreadsheet (digital appendix 7a4). 
Appendix $5 \mathrm{~b}$. Modifications to the tallgrass baseline model elicitation spreadsheet to derive values for the alternative models.

[Modifications are targeted at the distinctive aspects of each alternative model relative to the baseline model. Below, we summarize the conceptual model of all six models, starting with the baseline model, Model 3.

Additionally, for Models 1, 2, 4, 5, and 6 we pinpoint the distinctive feature of each model compared with Model 3 and describe how the values of the baseline elicitation spreadsheet were modified to accurately represent the conceptual models of each alternative model. The elicitation spreadsheets for all tallgrass models are located in appendixes $4 \mathrm{~b} 1-4 \mathrm{~b} 6$. Burn=burn within window, Graze $=$ graze within window, $\mathrm{SB}=$ smooth brome, $\mathrm{KB}=$ Kentucky bluegrass, $\mathrm{CO}=$ co-dominant smooth brome and Kentucky bluegrass, $\mathrm{RM}=$ remainder, $\mathrm{NP}=$ native prairie]

Model 3 (Baseline Model): Invader Type, Defoliation Level

Invader Type

- Management actions are differentially effective depending on the dominant invader, as follows:

- SB: Burn $>$ Graze $>$ Defoliate $>$ Rest

- CO: Burn $>$ Graze $>$ Defoliate $>$ Rest

- KB: Burn $>$ Graze $>$ Defoliate $>$ Rest

○ RM: Burn $=$ Graze $=$ Defoliate $>$ Rest

Defoliation Level

- Past management history affects the effect of current management actions. Actions have different effectiveness depending on whether the defoliation level is low or high, as follows:

- Rest is less detrimental under a high defoliation level than under a low defoliation level.

- At a low native cover (that is, 20 percent NP), active management actions are more effective under a high defoliation level than under a low defoliation level.

Invasion Level

- Management action effectiveness is not density-dependent.

\section{Model 1: Not state-based}

\section{Upper section of spreadsheet for change in native cover} Invader Type

- Effectiveness of management actions is not invader-specific; all forms of disturbance are equivalent and better than doing nothing (that is, rest).

- SB, CO, KB, RM: Burn $=$ Graze $=$ Defoliate $>$ Rest

Modification to baseline model elicitation spreadsheet values

- Rest: We set the values for rest to the average of the rest values over all four dominance types ( $\mathrm{SB}, \mathrm{CO}, \mathrm{KB}, \mathrm{RM})$.

- We averaged across the native cover starting levels (that is, across 80 percent NP and 20 percent NP).

- For the low defoliation level, we averaged across the low values.

- For the high defoliation level, we averaged across the low and the high values.

- Burn, Graze, Defoliate: We set the values for burn, graze, and defoliate equal to the average of the three actions over all four dominance types.

- For low defoliation level, we averaged across native cover starting levels (that is, across 80 percent NP and 20 percent NP).

- For high defoliation level, see below. 
Defoliation Level

- Past management history does not affect management effectiveness.

$>$ Modification to baseline model elicitation spreadsheet values

- Burn, Graze, Defoliate

- For high defoliation level, we set burn, graze, and defoliate equal to rest (at 80 percent NP and 20 percent NP).

Invasion Level

- For low defoliation level, see above.

- Management action effectiveness is not density-dependent.

$>$ Modification to baseline model elicitation spreadsheet values

- No modification necessary.

\section{Lower section of spreadsheet for change in dominance}

$>$ Modification to baseline model elicitation spreadsheet values-Dominance section

- Using the dominance spreadsheet, we changed rest probabilities and management action effects according to the values in the upper section of the spreadsheet (digital appendix $7 \mathrm{~b} 2)$.

- Modified so does not recognize a difference between SB, KB, or RM (because not statebased).

Model 2: Invader Type

\section{Upper section of spreadsheet for change in native cover}

Invader Type

- Management actions are differentially effective depending on the dominant invader.

$>$ Modification to baseline model elicitation spreadsheet values

Defoliation Level

○ No modification necessary.

- Past management history does not affect management effectiveness.

$>$ Modification to baseline model elicitation spreadsheet values

- At 80 percent NP, low and High Defoliation level

- Rest: Set rest high values equal to the rest low values for each dominance type.

- Burn, Graze, Defoliate: No change to burn, graze, or defoliate; the low and high values for each of these actions are already equal at 80 percent NP.

- At 20 percent NP, low and high defoliation level

- Rest: Set rest high values equal to the rest low values for each dominance type.

- Burn, Graze, Defoliate: Set burn, graze, and defoliate high values equal to the low values for each action at each dominance type.

Invasion Level

- Management action effectiveness is not density-dependent.

$>$ Modification to baseline model elicitation spreadsheet values

○ No modification necessary.

\section{Lower section of spreadsheet for change in dominance}

$>$ Modification to baseline model elicitation spreadsheet values-Dominance section 
- Using the dominance spreadsheet, we changed rest probabilities and management action effects according to the values in the upper section of the spreadsheet (digital appendix $7 b 3)$.

Model 4: Invader Type, Defoliation Level, Invasion Level

\section{Upper section of spreadsheet for change in native cover}

Invader Type

- Management actions are differentially effective depending on the dominant invader.

$>$ Modification to baseline model elicitation spreadsheet values

○ No modification necessary.

Defoliation Level

- Past management history does affect management effectiveness.

$>$ Modification to baseline model elicitation spreadsheet values

○ No modification necessary.

Invasion Level

$\circ$ Effectiveness of management action is density dependent.

- Effectiveness declines as the level of invasion increases.

- At 20 percent NP, active management actions are no more effective than rest.

$>$ Modification to baseline model elicitation spreadsheet values

- At 20 percent NP, low defoliation level

- Set burn, graze, and defoliate equal to the rest values within each dominance type.

$\circ$ At 20 percent NP, high defoliation level

- Set burn, graze, and defoliate to a 50|50 split above and below the bar.

\section{Lower section of spreadsheet for change in dominance}

$>$ Modification to baseline model elicitation spreadsheet values-Dominance section

- Using the dominance spreadsheet, we changed management action effects according to the values in the upper section of the spreadsheet (digital appendix 7b4).

Model 5: Invader Type (Graze = Burn if SB Dominant), Defoliation Level

\section{Upper section of spreadsheet for change in native cover}

Invader Type

- Management actions are differentially effective depending on the dominant invader, as follows:

○ SB: Burn $=$ Graze $>$ Defoliate $>$ Rest

- CO: Burn $>$ Graze $>$ Defoliate $>$ Rest

O KB: Burn $>$ Graze $>$ Defoliate $>$ Rest

O RM: Burn $=$ Graze $=$ Defoliate $>$ Rest

Modification to baseline model elicitation spreadsheet values

- In all quadrants (that is, 80 percent NP, Low; 80 percent NP, High; 20 percent NP, Low; 20 percent NP, High):

- For SB dominance, set graze values equal to the burn values.

- For CO dominance, set graze values halfway between the graze values for SB dominant and the graze values for KB dominance.

Defoliation Level

- Past management history does affect management effectiveness. 
Modification to baseline model elicitation spreadsheet values

- No modification necessary.

Invasion Level

- Management action effectiveness is not density-dependent.

$>$ Modification to baseline model elicitation spreadsheet values

$\circ$ No modification necessary.

\section{Lower section of spreadsheet for change in dominance}

$>$ Modification to baseline model elicitation spreadsheet values-Dominance section

- Using the dominance spreadsheet, we changed management action effects according to the values in the upper section of the spreadsheet (digital appendix 7b5).

Model 6: Invader Type (Defoliate $=$ Rest), Defoliation Level

\section{Upper section of spreadsheet for change in native cover} Invader Type

- Management actions are differentially effective depending on the dominant invader, as follows:

- SB: Burn $>$ Graze $>$ Defoliate $=$ Rest

- CO: Burn $>$ Graze $>$ Defoliate $=$ Rest

- KB: Burn $>$ Graze $>$ Defoliate $=$ Rest

- RM: Burn $=$ Graze $>$ Defoliate $=$ Rest

Modification to baseline model elicitation spreadsheet values

- For low defoliation (80 percent NP and 20 percent NP)

- Set the values for defoliate equal to the values for rest, for all dominance types.

- For high defoliation (80 percent NP and 20 percent NP)

- Set the values for defoliate to a 50|50 split above and below the bar, for all dominance types.

Defoliation Level

- Past management history does affect management effectiveness.

$>$ Modification to baseline model elicitation spreadsheet values

○ No modification necessary.

Invasion Level

- Management action effectiveness is not density-dependent.

$>$ Modification to baseline model elicitation spreadsheet values

$\circ$ No modification necessary.

\section{Lower section of spreadsheet for change in dominance}

$>$ Modification to baseline model elicitation spreadsheet values-Dominance section

- Using the dominance spreadsheet, we changed management action effects according to the values in the upper section of the spreadsheet (digital appendix 7b6). 
Appendix 6. Partial controllability.

[Individual responses per management unit. Management units are the lowest level of a hierarchy that includes complex (com), organization (org), group (grp), and unit (uni). This hierarchy was used to analyze the individual responses to account for hierarchical effects among levels (com id, org_id, grp_id, and uni_id). Values represent the probability that a recommended treatment will be implemented on a given management unit. The possible treatments include rest, graze, burn, and burn/graze $(B / G)$ for mixed-grass units and rest, graze within window, burn within window, and defoliate for tallgrass units. $\mathrm{r}=$ rest, $\mathrm{g}=$ graze or graze within window, $\mathrm{b}=$ burn or burn within window, $\mathrm{x}=$ burn/graze or defoliate, and $\mathrm{h}=\mathrm{hay}$. Where two codes appear, for example, 'rr' or 'rg', the first letter corresponds to the recommended treatment and the second letter to the implemented treated. The gray-filled columns 'rh', 'gh', 'bh', and 'xh' represent responses for implementing a hay action; because hay treatments are no longer recognized as one of the four treatment types, the probabilities indicated for hay were allocated among the other treatments (in the columns to the left) in proportion to the probability indicated for those treatments. There are responses for 119 units: 81 mixed-grass and 38 tallgrass. Cells outlined in a box indicate a recommended action that is not feasible for a particular unit and will never be implemented]

\begin{tabular}{|c|c|c|c|c|c|c|c|c|c|c|c|c|c|c|c|c|c|c|c|c|c|c|c|c|c|c|c|c|}
\hline \multirow{2}{*}{\begin{tabular}{|l|} 
Grass \\
Type \\
\end{tabular}} & \multicolumn{4}{|c|}{ Unit Hierarchy } & \multirow{2}{*}{\multicolumn{4}{|c|}{\begin{tabular}{|c|} 
Hierarchy indices \\
com_id org_id grp_id uni_id
\end{tabular}}} & \multicolumn{4}{|c|}{ Rest } & \multicolumn{4}{|c|}{ Graze } & \multicolumn{4}{|c|}{ Burn } & \multicolumn{4}{|c|}{$B / G$ or Defoliate } & \multicolumn{4}{|c|}{ Hay } \\
\hline & complex & org & group & unit & & & & & $\mathrm{rr}$ & $\mathrm{rg}$ & $r b$ & $r x$ & $\mathrm{gr}$ & gg & gb & gx & br & bg & bb & bx & $\mathbf{x r}$ & $\mathrm{xg}$ & $x \mathbf{x b}$ & $\mathbf{x x}$ & & $\mathrm{gh}$ & bh & $\mathrm{xh}$ \\
\hline & ARROWWOOD COMPLEX & ARROWWOOD NWR & G14 & G14 Pasture 1 & 1 & 1 & 1 & 1 & + & 0 & 0 & 0 & 0.125 & 0.125 & 0.75 & 0 & 0.25 & 0 & 0.75 & 0 & 0.25 & 0 & 0.75 & To & & 0 & 0 & 0 \\
\hline Mixed & ARROWWOOD COMPLEX & ARROWWOOD NWR & G14 & G14 Pasture 2 & 1 & 1 & 1 & 2 & 1 & 0 & 0 & 0 & 0.125 & 0.125 & 0.75 & 0 & 0.25 & 0 & 0.75 & 0 & 0.25 & & 0.75 & 0 & & & 0 & 0 \\
\hline Mixed & ARROWWOOD COMPLEX & ARROWWOOD NWR & G26 & G26 Paddock 1 & 1 & 1 & 2 & 3 & 0.75 & 0.25 & 0 & 0 & 0 & 1 & 0 & 0 & 0 & 0.125 & 0.75 & 0.125 & 0 & 0.125 & 0.125 & & & & 0 & 0 \\
\hline Mixed & ARROWWOOD COMPLEX & RROWWOOD NWR & G26 & G26 Paddock 2 & 1 & 1 & 2 & 4 & 0.75 & 0.25 & 0 & 0 & 0 & 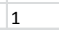 & 0 & 0 & 0 & 0.125 & 0.75 & 0.125 & 0 & 0.125 & 0.125 & 0.75 & & & 0 & 0 \\
\hline Mixed & ARROWWOOD COMPLEX & RROWWOOD NWR & G26 & G26 Paddock 3 & 1 & 1 & 2 & 5 & 0.75 & 0.25 & 0 & 0 & 0 & 1 & 0 & 0 & 0 & 0.125 & 0.75 & 0.125 & 0 & 0.125 & 0.125 & 0.75 & & 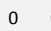 & 0 & 0 \\
\hline Mixed & ARROWWOOD COMPLEX & ARROWWOOD NWR & G26 & G26 Paddock 4 & 1 & 1 & 2 & 6 & 0.75 & 0.25 & 0 & 0 & 0 & 1 & 0 & 0 & 0 & 0.125 & 0.75 & 0.125 & 0 & 0.125 & 0.125 & 0.75 & & 0 & 0 & 0 \\
\hline Mixed & ARROWWOOD COMPLEX & ARROWWOOD NWR & G28 & 628 & 1 & 1 & 3 & 7 & 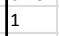 & 0 & 0 & 0 & 0 & 0 & ]$_{1}$ & 0 & 0 & 0 & 1 & 0 & 0 & 0 & 1 & 0 & & 0.5 & 0 & 0.25 \\
\hline Mixed & ARROWWOOD COMPLEX & EDDY COUNTY WPA & Have & Haven Paddock 4 & 1 & 2 & 4 & 8 & 0.5 & 0.5 & 0 & 0 & 0 & 1 & 0 & 0 & 10 & 0.125 & 0.75 & 0.125 & 0 & 0.125 & 0.125 & 0.75 & & & 0 & 0 \\
\hline Mixed & ARROW & YWPA & Have & Haven $\mathrm{P}$ & 1 & 2 & 4 & 9 & 0.5 & 0.5 & 0 & 0 & 10 & 1 & 0 & 0 & 10 & 0.125 & 0.75 & 0.125 & 0 & 0.125 & 0.125 & 0.75 & & 0 & 0 & 0 \\
\hline Mixed & ARROWWOOD COMPLEX & FOSTER COUNTY WPA & Topp & Topp East Paddock & 1 & 3 & 5 & 10 & 0.75 & 0.25 & 0 & 0 & 0 & 1 & 0 & 0 & 0 & 0.125 & 0.75 & 0.125 & 0 & 0.125 & 0.125 & 0.75 & & 0 & 0 & 0 \\
\hline Mixed & ARROWWOOD COMPLEX & FOSTER COUNTY WPA & Topp & Topp West Paddock & 1 & 3 & 5 & 11 & 0.75 & 0.25 & 0 & 0 & 0 & 1 & 0 & 0 & 0 & 0.125 & 0.75 & 0.125 & 0 & 0.125 & 0.125 & 0.75 & 0 & 0 & 0 & 0 \\
\hline Mixed & ARROWWOOD COMPLEX & GRIGGS COUNTY WPA & Wogs & Wogsland & 1 & 4 & 6 & 12 & 1 & 0 & 0 & 0 & 0.2 & 0.8 & 0 & 0 & 0.429 & 0 & 0.571 & 0 & 0.1 & 0.5 & 0.1 & 0.3 & & 0 & 0.3 & \\
\hline Mixed & ARROWWOOD COMPLEX & STUTSMAN COUNTY WPA & Odeg & Odegaard & 1 & 5 & 7 & 13 & 1 & 0 & 0 & 0 & 0 & 1 & 0 & 0 & 0 & 0 & 1 & 0 & 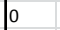 & 0 & 0 & 1 & & & 0 & 0 \\
\hline Mixed & ARROWWOOD COMPLEX & STUTSMAN COUNTY WPA & Winb & Winberg Unit 1 & 1 & 5 & 8 & 14 & 1 & 0 & 0 & 0 & 0 & 1 & 0 & 0 & 0 & 0 & 1 & 0 & 0 & 0 & 0 & 1 & & & & 0 \\
\hline Mixed & ARROWWOOD COMPLEX & STUTSMAN COUNTY WPA & Wood & Woodworth Station Unit 7 & 1 & 5 & 9 & 15 & 1 & 0 & 0 & 0 & 0 & 1 & 0 & 0 & 0 & 0 & 1 & 0 & 0 & 0 & 0 & 1 & 0 & 0 & 0 & 0 \\
\hline Mixed & ARROWWOOD COMPLEX & WELLS COUNTY WPA & Fred & Frederick & 1 & 6 & 10 & 16 & 1 & 0 & 0 & 0 & 0 & 1 & 0 & 0 & 0 & 0 & 1 & 0 & 0 & 0 & 0 & 1 & | & 0 & 0 & 0 \\
\hline Mixed & AUDUBON COMPLEX & MCLEAN COUNTY WPA & Koei & Koeing Section Line Slough Unit & 2 & 7 & 11 & 17 & 0.65 & 0.2 & 0.15 & 0 & 0.2 & 0.6 & 0.15 & 0.05 & 0.2 & 0.3 & 0.4 & 0.1 & 0.2 & 0.35 & 0.3 & 0.15 & & 0 & 0 & 0 \\
\hline Mixed & AUDUBON COMPLEX & JNTY WPA & Otis & Otis Unit 5A & 2 & 7 & 12 & 18 & 0.65 & 0.2 & 0.05 & 0.1 & 0.2 & 0.7 & 0.1 & 0 & 0.2 & 0.4 & 0.4 & $\cos _{2}$ & 0.2 & 0.4 & 0.2 & & & & & \\
\hline Mixed & AUDUBON COMPLEX & CLEAN COUNTY WPA & Otis & Otis U & 2 & 7 & 12 & 19 & 0.65 & 0.2 & 0.05 & 0.1 & 0.2 & 0.7 & 0.1 & 0 & 0.2 & 0.4 & 0.4 & 0 & 0.2 & 0.4 & 0.2 & 0.2 & & & & \\
\hline Mixed & AUDUBON COMPLEX & MCLEAN COUNTY WPA & Otis & Otis Unit $8 \mathrm{~N}$ & 2 & 7 & 12 & 20 & 0.65 & 0.2 & 0.05 & 0.1 & 0.2 & 0.7 & 0.1 & 0 & 0.2 & 0.4 & 0.4 & 0 & 0.2 & 0.4 & 0.2 & 0.2 & & 0 & 0 & 0 \\
\hline Mixed & AUDUBON COMPLEX & DUNTY WPA & Otis & Otis Unit 8S & 2 & 7 & 12 & 21 & 0.65 & 0.2 & 0.05 & 0.1 & 0.2 & 0.7 & 0.1 & 0 & 0.2 & 0.4 & 0.4 & 0 & 0.2 & 0.4 & 0.2 & 0.2 & & & & \\
\hline Mixed & AUDUBON COMPLEX & SHERIDAN COUNTY WPA & Lash & Lasher Unit A & 2 & 8 & 13 & 22 & 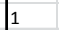 & 0 & 0 & & 0.1 & 0.7 & 0.1 & 0.1 & 0.1 & 0.1 & 0.7 & 0.1 & 0.1 & 0.1 & 0.1 & 0.7 & & 0 & & \\
\hline Mixed & AUDUBON COMPLEX & SHERIDAN COUNTY WPA & Lash & Lasher Unit B & 2 & 8 & 13 & 23 & 1 & 0 & 0 & 0 & 0.1 & 0.7 & 0.1 & 0.1 & 0.1 & 0.1 & 0.7 & 0.1 & 0.1 & 0.1 & 0.1 & 0.7 & & 0 & 0 & 0 \\
\hline Mixed & AUDUBON & UTY WPA & Pete & Peters & 2 & 9 & 14 & 24 & 1 & 0 & 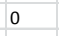 & 0 & 0.1 & 0.7 & 0.1 & 0 & 0.1 & 0.1 & 0. & 0.1 & 0.1 & 0.1 & 0.1 & 0.7 & & & 0 & 0 \\
\hline Mixed & AUDUBON COMPLEX & NTY WPA & Pete & Peters & 2 & 9 & 14 & 25 & $\left.\right|^{1}$ & 0 & 0 & 0 & 0.1 & 0.7 & 0.1 & 0.1 & 0.1 & 0.1 & 0.7 & 0.1 & 0.1 & 0.1 & 0.1 & 0.7 & & 0 & 0 & 0 \\
\hline Mixed & AUDUBON COMPLEX & D COUNTY WPA & Pete & Peterso & 2 & 9 & 14 & 26 & & 0 & 0 & 0 & 0.1 & 0.7 & 0.1 & 0.1 & 0.1 & 0.1 & 0.7 & 0.1 & 0.1 & 0.1 & 0.1 & 0.7 & & 0 & & \\
\hline Mixed & AUDUBON COMPLEX & WARD COUNTY WPA & Pete & Peterson SW & 2 & 9 & 14 & 27 & 1 & 0 & 0 & 0 & 0.1 & 0.7 & 0.1 & 0.1 & 0.1 & 0.1 & 0.7 & 0.1 & 0.1 & 0.1 & 0.1 & 0.7 & & 0 & 0 & 0 \\
\hline Mixed & BENTON LAKE WMD & TOOLE COUNTY WPA & Ehli & Ehli & 3 & 10 & 15 & 28 & 2 & 0 & 0 & 0 & 0 & 0.75 & 0.25 & 0 & 0.25 & 0 & 0.75 & 0 & 0.5 & 0 & 0 & 0.5 & & 0 & 0 & 0 \\
\hline Mixed & BENTON LAKE WMD & TOOLE COUNTY WPA & Furn & Furnell & 3 & 10 & 16 & 29 & 1 & 0 & 0 & 0 & 0 & 1 & 0 & 0 & 0 & 1 & 0 & ]o & 0 & 1 & 0 & $\Gamma$ & & 0 & & 0 \\
\hline Mixed & DEVILS LAKE WMD & BENSON COUNTY WPA & Mela & Melass Sout & 4 & 11 & 17 & 30 & 1 & 0 & 0 & 0 & 0 & 1 & 0 & 0 & 0 & 0 & 1 & 0 & 0 & 0 & 0 & 1 & & 0 & 0 & 0 \\
\hline Mixed & DEVILS LAKE WMD & SULLYS HILL NATIONAL GAME PRESERVE & Sull & Sullys Hills Native Prairie & 4 & 12 & 18 & 31 & 1 & 0 & 0 & 0 & 0 & 1 & 0 & 0 & 0 & 0 & 1 & 0 & 0 & 0 & 0 & 1 & & 0 & 0 & 0 \\
\hline Mixed & $\begin{array}{ll}\text { HURON WMD } \\
\end{array}$ & BUFFALO COUNTY WPA & Mill & Mills Unit 2 & 5 & 13 & 19 & 32 & 0.9 & 0.04 & 0.05 & 0.01 & 0.03 & 0.85 & 0.02 & 0.1 & 0.2 & 0.05 & 0.55 & 0.2 & 0.12 & 0.12 & 0.01 & 0.75 & & 0 & 0 & 0 \\
\hline Mixed & $\begin{array}{l}\text { HURON WMD } \\
\text { HUR }\end{array}$ & HAND COUNTY WPA & Camp & Campbell Unit 2 & 5 & 14 & 20 & 33 & 0.9 & 0.04 & 0.05 & 0.01 & 0.03 & 0.85 & 0.02 & 0.1 & 0.2 & 0.05 & 0.55 & 0.2 & 0.12 & 0.12 & 0.01 & 0.75 & & 0 & 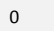 & 0 \\
\hline Mixed & & & Mill & Mille & 5 & 14 & 21 & 34 & 0.9 & 0.04 & 0.05 & 0.01 & 0.03 & 0.85 & 0.02 & & 0.2 & 0.05 & & 0.2 & & 0.12 & 0.01 & 0.75 & & & & \\
\hline & & & & & & 14 & 22 & 35 & & & & & & & 007 & & & & & & & & & & & & & \\
\hline & $\begin{array}{l}\text { d HURON WMD } \\
\end{array}$ & HAND COUNTY WPA & Slun & Slunecka Unit 4 & 5 & 14 & 22 & 36 & 0.9 & 0.04 & 0.05 & & 0.03 & 0.85 & 0.02 & & 0.2 & 0.05 & 0.55 & 0.2 & 0.12 & 0.12 & 0.01 & & & & & 0 \\
\hline
\end{tabular}




\begin{tabular}{|c|c|c|c|c|c|c|c|c|c|c|c|c|c|c|c|c|c|c|c|c|c|c|c|c|c|c|c|c|}
\hline \multirow{2}{*}{$\begin{array}{l}\text { Grass } \\
\text { Type } \\
\end{array}$} & \multicolumn{4}{|c|}{ Unit Hierarchy } & \multicolumn{4}{|c|}{ Hierarchy indices } & \multicolumn{4}{|c|}{ Rest } & \multicolumn{4}{|c|}{ Graze } & & & urn & & & $B / G$ or $D$ & Defoliat & & & & Hay & \\
\hline & complex & org & group & unit & com_i & org_ic & grp_id & uni_id & $\mathrm{rr}$ & 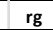 & $\mathrm{rb}$ & $r x$ & $\mathrm{gr}$ & gg & gb & gx & br & bg & bb & bx & $\mathrm{xr}$ & $\mathbf{x g}$ & $\mathrm{xb}$ & $x x$ & & gh & bh & $\mathrm{xh}$ \\
\hline & \begin{tabular}{|l|} 
HURON WMD \\
\end{tabular} & HAND COUNTY WPA & VenJ & VenJohn Unit 1 & 5 & 14 & 23 & 37 & 0.9 & 0.04 & 0.05 & 0.01 & 0.03 & 0.85 & 0.02 & 0.1 & 0.2 & 0.05 & 0.55 & 0.2 & 0.12 & 0.12 & 0.01 & 0.75 & 0 & 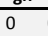 & 0 & \\
\hline Mixed & HURON WMD & HYDE COUNTY WPA & Cowa & Cowan Unit 4 & 5 & 15 & 24 & 38 & 0.9 & 0.04 & 0.05 & 0.01 & 0.03 & 0.85 & 0.02 & 0.1 & 0.2 & 0.05 & 0.55 & 0.2 & 0.12 & 0.12 & 0.01 & 0.75 & & & & \\
\hline Mixed & HURON WMD & HYDE COUNTY WPA & Cowa & Cowan Unit 6 & 5 & 15 & 24 & 39 & 0.9 & 0.04 & 0.05 & 0.01 & 0.03 & 0.85 & 0.02 & 0.1 & 0.2 & 0.05 & 0.55 & 0.2 & 0.12 & 0.12 & 0.01 & 0.75 & & & & \\
\hline Mixed & HURON WMD & HYDE COUNTY WPA & Hart & Harter Unit 6 & 5 & 15 & 25 & 40 & 0.9 & 0.04 & 0.05 & 0.01 & 0.03 & 0.85 & 0.02 & 0.1 & 0.2 & 0.05 & 0.55 & 0.2 & 0.12 & 0.12 & 0.01 & 0.75 & & 0 & & \\
\hline Mixed & HURON WMD & JERAULD COUNTY WPA & Wint & Winter & 5 & 16 & 26 & 41 & 0.9 & 0.04 & 0.05 & 0.01 & 0.03 & 0.85 & 0.02 & 0.1 & 0.2 & 0.05 & 0.55 & 0.2 & 0.12 & 0.12 & 0.01 & 0.75 & & & 0 & \\
\hline Mixed & KULM WMD & LA MOURE COUNTY WPA & Corn & Cornell $1-2$ & 6 & 17 & 27 & 42 & 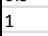 & 0 & 0 & 0 & 0.2 & 0.8 & 0 & 0 & 0.1 & 0.5 & 0.25 & 0.15 & 0.1 & 0.55 & 0.1 & 0.25 & & 0.5 & 0 & \\
\hline Mixed & KULM WMD & LA MOURE COUNTY WPA & Corn & Cornell 3 & 6 & 17 & 27 & 43 & 1 & 0 & 0 & 0 & 0.2 & 0.8 & 0 & 0 & 0.1 & 0.5 & 0.25 & 0.15 & 0.1 & 0.55 & 0.1 & 0.25 & & 0.5 & & \\
\hline Mixed & KULM WMD & LOGAN COUNTY WPA & Krol & Kroll 1 & 6 & 18 & 28 & 44 & 1 & 0 & 0 & 0 & 0.25 & 0.75 & 0 & 0 & 0.25 & 0.5 & 0.15 & 0.1 & 0.25 & 0.5 & 0.1 & 0.15 & & & & \\
\hline Mixed & KULM WMD & LOGAN COUNTY WPA & Maye & Mayer 2 & 6 & 18 & 29 & 45 & 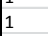 & 0 & 0 & 0 & 0.1 & 0.9 & 0 & 0 & 0.1 & 0.55 & 0.25 & 0.1 & 0.1 & 0.55 & 0.1 & 0.25 & & 0 & 0 & \\
\hline Mixed & KULM WMD & LOGAN COUNTY WPA & Maye & Mayer 3 & 6 & 18 & 29 & 46 & & 0 & 0 & 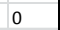 & 0.1 & 0.9 & 0 & 0 & 0.1 & 0.55 & 0.25 & 0.1 & 0.1 & 0.55 & 0.1 & 0.25 & & 0 & 0 & \\
\hline Mixed & KULM WMD & LOGAN COUNTY WPA & Maye & Mayer 4 & 6 & 18 & 29 & 47 & & 0 & 0 & 0 & 0.1 & 0.9 & 0 & 0 & 0.1 & 0.55 & 0.25 & 0.1 & 0.1 & 0.55 & 0.1 & 0.25 & & & 0 & \\
\hline Mixed & KULM WMD & MCINTOSH COUNTY WPA & Geis & Geiszler 1 & 6 & 19 & 30 & 48 & 0.9 & 0.1 & 0 & 0 & 0 & 1 & 0 & 0 & 0.1 & 0.65 & 0.25 & 0 & 0.1 & 0.5 & 0.15 & 0.25 & & 0 & 0 & \\
\hline Mixed & KULM WMD & MCINTOSH COUNTY WPA & Geis & Geiszler 2 & 6 & 19 & 30 & 49 & 0.9 & 0.1 & 0 & 0 & 0.1 & 0.9 & 0 & 0 & 0.1 & 0.65 & 0.25 & 0 & 0.1 & 0.5 & 0.15 & 0.25 & & & 0 & \\
\hline Mixed & KULM WMD & MCINTOSH COUNTY WPA & Geis & Geiszler 3 & 6 & 19 & 30 & 50 & 0.9 & 0.1 & 0 & 0 & 0.1 & 0.9 & 0 & 0 & 0.1 & 0.65 & 0.25 & 0 & 0.1 & 0.5 & 0.15 & 0.25 & & 0 & 0 & \\
\hline Mixed & KULM WMD & MCINTOSH COUNTY WPA & Geis & Geiszler 4 & 6 & 19 & 30 & 51 & 0.9 & 0.1 & 0 & 0 & 0.1 & 0.9 & 0 & 0 & 0.1 & 0.65 & 0.25 & 0 & 0.1 & 0.5 & 0.15 & 0.25 & & 0 & 0 & \\
\hline Mixed & LAKE ANDES NWR & AURORA COUNTY WPA & Fost & Foster & 7 & 20 & 31 & 52 & 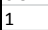 & 0 & 0 & 5 & 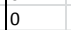 & 1 & 0 & 0 & 0 & 0.333 & 0.667 & 0 & 0 & 0.5 & . & 0.5 & & & & \\
\hline Mixed & LAKE ANDES NWR & BONHC & Hieb & Hieb & 7 & 21 & 32 & 53 & 1 & 0 & 0 & 0 & 0 & 1 & 0 & 0 & 0 & 0.333 & 0.667 & 0 & 0 & 0 & 0 & 0.5 & & 0.2 & & \\
\hline Mixed & $\begin{array}{l}\text { LAKE ANDES NWR } \\
\text { L }\end{array}$ & DOUGLAS COUNTY WPA & Denn & Denning & 7 & 22 & 33 & 54 & 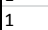 & 0 & 0 & 0 & 0 & 1 & 0 & 0 & 0 & 0.333 & 0.667 & 0 & 0 & 0.5 & 0 & 0.5 & & 0 & & \\
\hline Mixed & LONG LAKE WMD & BURLEIGH COUNTY WPA & Crim & Crimmins NE Grazing Unit & 8 & 23 & 34 & 55 & 1 & 0 & 0 & 0 & 0.15 & 0.75 & 0.1 & 0 & 0.25 & 0.25 & 0.5 & 0 & 0.2 & 0.4 & 0.2 & 0.2 & 0 & 0 & 0 & \\
\hline Mixed & LONG LAKE WMD & BURLEIGH COUNTY WPA & Rath & Rath WPA-\#1 Grazing Unit & 8 & 23 & 35 & 56 & 1 & 0 & 0 & 0 & 0.15 & 0.75 & 0.1 & 0 & 0.2 & 0.3 & 0.5 & 0 & 0.25 & 0.4 & 0.1 & 0.25 & & 0 & 0 & \\
\hline Mixed & LONG LAKE WMD & KIDDER COUNTY WPA & Thac & Thacker WPA - North & 8 & 24 & 36 & 57 & $I^{2}$ & 0 & 0 & 0 & 0.2 & 0.8 & 0 & 0 & 0.5 & 0.5 & 0 & ]$_{0}^{0}$ & 0.5 & 0.5 & 0 & 0 & & 0 & 0 & \\
\hline Mixed & LONG LAKE WMD & KIDDER COUNTY WPA & Thac & Thacker WPA - South & 8 & 24 & 36 & 58 & 1 & 0 & 0 & 0 & 0.2 & 0.8 & 0 & 0 & 0.5 & 0.5 & 0 & $\int_{0}$ & 0.5 & 0.5 & 0 & 0 & & 0 & 0 & \\
\hline Mixed & LONG LAKE WMD & LONG LAKE NWR & G-12 & G-12A East & 8 & 25 & 37 & 59 & $\tau^{-}$ & 0 & 0 & 0 & 0.2 & 0.75 & 0.05 & 0 & 0.3 & 0.4 & 0.3 & 0 & 0.2 & 0.5 & 0.1 & 0.2 & 0 & 0 & 0 & \\
\hline Mixed & LOSTWOOD COMPLEX & LAKE ZAHL NWR & Lake & Lake Zahl 7 & 9 & 26 & 38 & 60 & $i_{1}$ & 0 & 0 & 0 & 0.2 & 0.8 & 0 & 0 & 0.25 & 0 & 0.75 & 0 & 0.1 & 0.1 & 0.2 & 0.6 & & 0 & 0 & \\
\hline Mixed & LOSTWOOD COMPLEX & LOSTWOOD NWR & Wind & Windmill Sou & 9 & 27 & 39 & 61 & & 0 & 0 & 0 & 0.1 & 0.9 & 0 & 0 & 0.25 & 0.25 & 0.5 & 0 & 0.25 & 0.25 & 0.25 & 0.25 & & & & \\
\hline Mixed & $\begin{array}{l}\text { LOSTWOOD CO } \\
\text { LOSW }\end{array}$ & MOUNT & Cote & Coteas & 9 & 2 & 40 & 62 & & 0 & 0 & 0 & 0.111 & 0.889 & 0 & 0 & 0.25 & 0 & 0.75 & 0 & 0.1 & 0.1 & 0.2 & 0.6 & & 0.1 & & \\
\hline Mixed & MEDICINE LAKE NWR COMPLEX & MEDICINE LAKE NWR & East & East ML Bridgerman & 10 & 29 & 41 & 63 & 0.9 & 0.05 & 0.05 & 0 & 0.65 & 0.25 & 0.05 & 0.05 & 0.15 & 0.2 & 0.5 & 0.15 & 0.05 & 0.35 & 0.1 & 0.5 & & 0 & 0 & \\
\hline Mixed & MEDICINE LAKE NWR COMPLEX & MEDICINE LAKE NWR & East & East ML LAKE 10 & 10 & 29 & 41 & 64 & 0.8 & 0.1 & 0.05 & 0.05 & 0.1 & 0.8 & 0.05 & 0.05 & 0.25 & 0.1 & 0.55 & 0.1 & 0.1 & 0.2 & 0.2 & 0.5 & & 0 & 0 & \\
\hline Mixed & MEDICINE LAKE NWR COMPLEX & MEDICINE LAKE NWR & Home & Homestead North & 10 & 29 & 42 & 65 & 0.9 & 0.05 & 0.05 & 0 & 0.05 & 0.75 & 0.05 & 0.15 & 0.05 & 0.05 & 0.7 & 0.2 & 0.05 & 0.25 & 0.1 & 0.6 & & & 0 & \\
\hline Mixed & MEDICINE LAKE N & MEDICINE LAKE NWR & Home & Homestead South & 10 & 29 & 42 & 66 & 0.95 & 0.02 & 0.02 & 0.01 & 0.05 & 0.85 & 0.05 & 0.05 & 0.05 & 0.1 & 0.6 & 0.25 & 0.05 & 0.3 & 0.15 & 0.5 & & 0 & 0 & \\
\hline Mixed & MEDICIN & & Ande & Ander: & 10 & 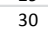 & 43 & 67 & 0.65 & 0.2 & 0.1 & 0.05 & 0.5 & 0.5 & 0 & 0 & 0.4 & 0.1 & 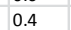 & 0.1 & 0.05 & 0.3 & 0.25 & 0.4 & & & & \\
\hline Mixed & MEDICINE LAKE NWR COMPLEX & SHERIDAN COUNY WPA & es & Gjesdal West & 10 & 30 & 44 & 68 & 0.65 & 0.2 & 0.1 & 0.05 & 0.5 & 0.5 & 0 & 0 & 0.4 & 0.1 & 0.4 & 0.1 & 0.05 & 0.3 & 0.25 & 0.4 & & 0 & 0 & \\
\hline Mixed & SAND LAKE COMPLEX & CAMPBELL COUNTY WPA & Coop & Cooper North & 11 & 31 & 45 & 69 & 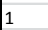 & 0 & 0 & 0 & 0 & 0.75 & 0.2 & 0.05 & & 0.2 & 0.75 & 0.05 & 0 & 0.5 & 0.25 & 0.25 & & 0 & 0 & \\
\hline Mixed & SAND LAKE COMPLEX & EDMUNDS COUNTY WPA & Mitz & Mitzel & 11 & 32 & 46 & 70 & 1 & 0 & 0 & 0 & 0 & 1 & 0 & 0 & 0 & 0.25 & 0.75 & 0 & 0 & 0.3 & 0.3 & 0.4 & & & 0 & \\
\hline Mixed & SAND LAKE COMPLEX & MCPHERSON COUNTY WPA & Char & Charley-Harley & 11 & 33 & 47 & 71 & 1 & 0 & 0 & 0 & 0 & 1 & 0 & 0 & 0.05 & 0.2 & 0.75 & 0 & 0 & 0.3 & 0.3 & 0.4 & & & 0 & \\
\hline Mixed & SOURIS RIVER BASIN COMPLEX & DES LACS NWR & HB 7 & $\mathrm{HB} 7$ & 12 & 34 & 48 & 72 & 0.8 & 0.2 & 0 & 0 & 0.1 & 0.7 & 0 & 0.2 & 0.3 & 0.1 & 0.5 & 0.1 & 0.2 & 0.2 & 0.2 & 0.4 & & 0 & & \\
\hline Mixed & SOURIS RIVER BAS & J. CLARK SALYER N & GLT & GLT PLC & 12 & 35 & 49 & 73 & 0.8 & 0.2 & 0 & 0 & 0.1 & 0.7 & 0 & 0.2 & 0.3 & 0.1 & 0.5 & 0.1 & 0.2 & 0.2 & 0.2 & 0.4 & & 0 & 0 & \\
\hline Mixed & SOURIS RIVER BAS & $\begin{array}{l}\text { J. CLARK SALYER NWR } \\
\text { S S }\end{array}$ & GLT & GLT PLOT C & 12 & 35 & 49 & 74 & 0.8 & 0.2 & 0 & 0 & 0.1 & 0.7 & 0 & 0.2 & 0.3 & 0.1 & 0.5 & 0.1 & 0.2 & 0.2 & 0.2 & 0.4 & & 0 & 0 & \\
\hline Mixed & SOURIS RIVER BASIN COMPLEX & J. CLARK SALYER NWR & Nels & Nelson Prairie 1 & 12 & 35 & 50 & 75 & & 0 & 0 & 0 & 0.1 & 0.7 & 0 & 0.2 & 0.3 & 0.1 & 0.5 & 0.1 & 0.3 & 0.2 & 0.2 & 0.3 & & & & \\
\hline Mixed & SOURIS RIVER BASIN COMPLEX & J. CLARK SALYER NWR & Nels & Nelson $\mathrm{Pr}$ & 12 & 35 & 50 & 76 & & 0 & 0 & 0 & 0.1 & 0.7 & 0 & 0.2 & 0.3 & 0.1 & 0.5 & 0.1 & 0.2 & 0.2 & 0.2 & 0.4 & & & & \\
\hline Mixed & SOURIS RIVER BASIN COMPLEX & J. CLARK SALYER NWR & Nels & Nelson Prairie 3 & 12 & 35 & 50 & 77 & $a_{1}^{2}$ & 0 & 0 & 0 & 0.1 & 0.7 & 0 & 0.2 & 0.3 & 0.1 & 0.5 & 0.1 & 0.2 & 0.2 & 0.2 & 0.4 & & & 0 & \\
\hline Mixed & $\begin{array}{l}\text { SOURIS RIVER B } \\
\text { SO }\end{array}$ & J. CLARK & Nels & Nelson & 13 & 35 & 50 & 78 & 1 & 0 & 0 & 0 & 0.1 & 0.7 & 0 & 0.2 & 0.3 & 0 & 0.5 & 0.1 & 0.2 & 0.2 & 0.2 & 0.4 & & 0 & & \\
\hline Mixed & SOURIS & MCHE & Kell & Keller Unit 1 & 1 & 36 & 51 & 79 & 0.6 & 0.4 & 0 & 0 & 0.1 & 0.8 & 0 & 0.1 & 0.2 & 0.3 & 0.4 & 0.1 & 0.1 & 0.5 & 0.1 & 0.3 & & 0 & 0 & \\
\hline Mixed & SOURIS RIVER BA & MCHENRY COUNTY WPA & Kell & Keller Unit 2 & 12 & 36 & 51 & 80 & 0.6 & 0.4 & 0 & 0 & 0.1 & 0.8 & 0 & 0.1 & 0.2 & 0.3 & 0.4 & 0.1 & 0.1 & 0.5 & 0.1 & 0.3 & & & 0 & \\
\hline Mixed & SOURIS RIVER BASIN COMPLEX & UPPER SOURIS NWR & $\mathrm{HB}-2$ & Ranch South & 12 & 37 & 52 & 81 & 0.8 & 0.2 & 0 & 0 & 0.1 & 0.7 & 0 & 0.2 & 0.3 & 0.1 & 0.5 & 0.1 & 0.1 & 0.3 & 0.3 & 0.3 & & & 0 & \\
\hline Tall & BIG STONE NWR & BIG STONE N & Lask & Laskow & 1 & 1 & 1 & 1 & 0.8 & 0.1 & 0 & 0.1 & 0.1 & 0.65 & 0 & 0.25 & 0.15 & 0.1 & 0.75 & 0 & 0.2 & 0.15 & 0 & 0.65 & & & & \\
\hline Tall & SAND LAKE COMPLEX & SPINK COL & Sand & Sanderson & 2 & 2 & 2 & 2 & & 0 & 0 & 0. & 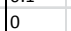 & 5. & 0 & 0 & 0 & 0.25 & 0.75 & 0 & 0 & 0 & 0 & 1 & & & & \\
\hline Tall & MADISO & DEUE & Mill & Miller & 3 & 3 & 3 & 3 & 1 & 0 & 0 & 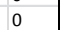 & 0.2 & 0.7 & 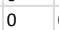 & 0.1 & 0.2 & 0 & 0.5 & 0.3 & 0.25 & 0.25 & 0.25 & 0.25 & & & & \\
\hline Tall & MADISON WMD & TY WPA & $\mathrm{pn}$ & IWPA & 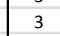 & 4 & 4 & 4 & $t^{2}$ & 0 & 0 & 0 & 0.2 & 0.7 & 0 & 0.1 & 0.2 & 0 & 0.5 & 0.3 & 0.25 & 0.25 & 0.25 & 0.25 & & & & \\
\hline Tall & MADISON WMD & MINNEHAHA COUNTY & Buff & Buffalo Lake 80 & 3 & 5 & 5 & 5 & ${ }_{1}$ & 0 & 0 & 0 & 0.2 & 0.5 & 0 & 0.3 & 0.2 & 0 & 0.5 & 0.3 & 0.25 & 0.25 & 0.25 & 0.25 & & & & \\
\hline Tall & DEVILS LAKE WMD & GRAND FORKS COUNTY WPA & Meki & Mekinock & 4 & 6 & 6 & 6 & 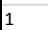 & 0 & 0 & 0 & & & & 0 & 0.1 & 0 & 0.5 & 0.4 & 0.1 & & 0.4 & 0.5 & & & & \\
\hline Tall & TEWAU & $\mathrm{RICHL}$ & & $\mathrm{Har}$ & 5 & 7 & 7 & 7 & & & 0 & & 0.2 & 0.1 & 0 & 0.7 & 0.2 & 0.1 & $1 \quad 0.1$ & 0.6 & 0.2 & 0.1 & & 0.7 & & & & \\
\hline Tall & & & & & 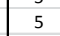 & 7 & 7 & $0^{\circ}$ & & 0 & 0 & & 0.2 & 0.1 & 0 & & & 0.1 & & 0.6 & & & & & & & & \\
\hline Tall & TEW & RIC & Hart & Hartleb & 5 & 7 & 7 & 9 & 1 & 0 & 0 & & 0.2 & 0.1 & 0 & $0.7 \mid$ & 0.2 & 0.1 & 0.1 & 0.6 & 0.2 & 0.1 & & & & & & \\
\hline Tall & TEWAUKON WMD & SARGENT & Gain & Gainor Unit A & 5 & 8 & 8 & 10 & 1 & 0 & 0 & & 0 & 0.3 & 0.1 & 0.6 & 0 & 0.3 & 0.1 & 0.6 & 0 & 0 & 0 & & & & & \\
\hline Tall & TEWAUKON WMD & SARGENT COUNTY WPA & Gain & Gainor Unit B & 5 & 8 & 8 & 11 & 1 & 0 & 0 & & 0 & 0.3 & 0.1 & 0.6 & 0 & 0.3 & \begin{tabular}{|l|l|}
3 & 0.1 \\
\end{tabular} & 0.6 & 0 & 0 & 0 & 0 & & & & \\
\hline Tall & TEWAUKON WMD & SARGENT COUNTY WPA & Krau & Krause & 5 & 8 & 9 & 12 & 1 & 0 & 0 & & 0 & 0.2 & 0.1 & 0 & 0 & 0.2 & 0.1 & 7 & 0 & 0 & & & & & & \\
\hline Tall & $\begin{array}{l}\text { TEWAUKON WMD } \\
\text { TEWA }\end{array}$ & TEWAUKON NWR & $\begin{array}{l}\text { Tewau } \\
\text { Tewa }\end{array}$ & Tewaukon NWR & 5 & 9 & 10 & $\begin{array}{l}12 \\
13\end{array}$ & 1 & 0 & 0 & & 0 & 0.3 & $\begin{array}{l}0.1 \\
0.1\end{array}$ & $\begin{array}{l}0.1 \\
0.6\end{array}$ & 0 & 0.2 & $\begin{array}{ll}0.1 \\
3 & 0.1\end{array}$ & $\begin{array}{ll}1 & 0.6 \\
1 & 0.6\end{array}$ & 0 & 0 & 0 & & & & & \\
\hline
\end{tabular}




\begin{tabular}{|c|c|c|c|c|c|c|c|c|c|c|c|c|c|c|c|c|c|c|c|c|c|c|c|c|c|}
\hline \multicolumn{4}{|c|}{ Unit Hierarchy } & \multicolumn{4}{|c|}{ Hierarchy indices } & \multicolumn{4}{|c|}{ Rest } & \multicolumn{4}{|c|}{ Graze } & \multicolumn{4}{|c|}{ Burn } & \multicolumn{4}{|c|}{$\mathrm{B} / \mathrm{G}$ or Defoliate } & \multicolumn{2}{|l|}{ Hay } \\
\hline complex & org & group & unit & com_i & org_ic & grp_ic & uni_id & $\mathrm{rr}$ & $\mathrm{rg}$ & rb & $r x$ & $\mathrm{gr}$ & $\mathrm{gg}$ & gb & $\mathrm{gx}$ & br & bg & bb & bx & $\mathrm{xr}$ & $\mathrm{xg}$ & $\mathrm{xb}$ & $x x$ & rh gh bh & $\mathrm{xh}$ \\
\hline WAUBAY NWR COMPLEX & CLARK COUNTY WPA & Warn & Warner Lake Paddock 5 & 6 & 10 & 11 & 14 & 1 & 0 & $\overline{0}$ & 0 & 0.1 & 0.6 & 0 & 0.3 & 0.1 & $\overline{0}$ & 0.3 & 0.6 & 0.1 & 0.1 & 0.1 & \begin{tabular}{c|c}
0.7 \\
\end{tabular} & & \\
\hline WAUBAY NWR COMPLEX & CODINGTON COUNTY WPA & Roe & Roe E & 6 & 11 & 12 & 15 & 1 & 0 & 0 & 0 & 0.1 & 0.6 & 0 & 0.3 & 0.1 & 0 & 0.3 & 0.6 & 0.1 & 0.1 & 0.1 & 0.7 & & \\
\hline WAUBAY NWR COMPLEX & CODINGTON COUNTY WPA & Roe & Roe F & 6 & 11 & 12 & 16 & 1 & 0 & 0 & 0 & 0.1 & 0.6 & 0 & 0.3 & 0.1 & 0 & 0.3 & 0.6 & 0.1 & 0.1 & 0.1 & 0.7 & & \\
\hline WAUBAY NWR COMPLEX & MARSHALL COUNTY WPA & Buff & Buffalo Lake & 6 & 12 & 13 & 17 & 1 & 0 & 0 & 0 & 0.1 & 0.6 & 0 & 0.3 & 0.1 & 0 & 0.3 & 0.6 & 0.1 & 0.1 & 0.1 & 0.7 & & \\
\hline WAUBAY NWR COMPLEX & MARSHALL COUNTY WPA & Buss & Buss Paddock 1 & 6 & 12 & 14 & 18 & 1 & 0 & 0 & 0 & 0.1 & 0.6 & 0 & 0.3 & 0.1 & 0 & 0.3 & 0.6 & 0.1 & 0.1 & 0.1 & 0.7 & & \\
\hline WAUBAY NWR COMPLEX & MARSHALL COUNTY WPA & Buss & Buss Paddock 2 & 6 & 12 & 14 & 19 & 1 & 0 & 0 & 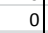 & 0.1 & 0.6 & 0 & 0.3 & 0.1 & 0 & 0.3 & 0.6 & 0.1 & 0.1 & 0.1 & 0.7 & & \\
\hline WAUBAY NWR COMPLEX & MARSHALL COUNTY WPA & Jens & Jensen East & 6 & 12 & 15 & 20 & 1 & 0 & 0 & 0 & 0.1 & 0.6 & 0 & 0.3 & 0.1 & 0 & 0.3 & 0.6 & 0.1 & 0.1 & 0.1 & 0.7 & & \\
\hline WAUBAY NWR COMPLEX & ROBERTS COUNTY WPA & Berw & Berward Paddock 4 & 6 & 13 & 16 & 21 & 1 & 0 & 0 & 0 & 0.1 & 0.6 & 0 & 0.3 & 0.1 & 0 & 0.3 & 0.6 & 0.1 & 0.1 & 0.1 & 0.7 & & \\
\hline WAUBAY NWR COMPLEX & ROBERTS COUNTY WPA & Berw & Berward Paddock 5 & 6 & 13 & 16 & 22 & 1 & 0 & 0 & 0 & 0.1 & 0.6 & 0 & 0.3 & 0.1 & 0 & 0.3 & 0.6 & 0.1 & 0.1 & 0.1 & 0.7 & & \\
\hline WAUBAY NWR COMPLEX & ROBERTS COUNTY WPA & Wike & Wike Paddock 1 & 6 & 13 & 17 & 23 & 1 & 0 & 0 & 0 & 0.1 & 0.6 & 0 & 0.3 & 0.1 & 0 & 0.3 & 0.6 & 0.1 & 0.1 & 0.1 & 0.7 & & \\
\hline WAUBAY NWR COMPLEX & ROBERTS COUNTY WPA & Wike & Wike Paddock 2 & 6 & 13 & 17 & 24 & 1 & 0 & 0 & 0 & 0.1 & 0.6 & 0 & 0.3 & 0.1 & 0 & 0.3 & 0.6 & 0.1 & 0.1 & 0.1 & 0.7 & & \\
\hline WINDOM WMD & COTTONWOOD COUNTY WPA & Des & Des Moines River WPA - North & 7 & 14 & 18 & 25 & 1 & 0 & 0 & 0 & 0.5[ & 0 & 0.5 & 0 & 0 & 0 & 1 & 0 & 0 & 0 & 0 & 1 & & \\
\hline WINDOM WMD & COTTONWOOD COUNTY WPA & Des & Des Moines River WPA - South & 7 & 14 & 18 & 26 & 1 & 0 & 0 & 0 & 0.5 & 0 & 0.5 & 0 & 0 & 0 & 1 & 0 & 0 & 0 & 0 & 1 & & \\
\hline DETROIT LAKES WMD & CLAY COUNTY WPA OF MINNESOTA & Hoyk & Hoykens WPA North & 8 & 15 & 19 & 27 & 1 & 0 & 0 & 0 & 0.9 & 0 & 0.1 & 0 & 0.3 & 0 & 0.1 & 0.6 & 0.3 & 0 & 0.1 & 0.6 & & \\
\hline DETROIT LAKES WMD & CLAY COUNTY WPA OF MINNESOTA & Jarv & Jarvis WPA & 8 & 15 & 20 & 28 & 1 & 0 & 0 & 0 & 0.7 & 0 & 0.3 & 0 & 0.3 & 0 & 0.3 & 0.4 & 0.3 & 0 & 0.1 & 0.6 & & \\
\hline DETROIT LAKES WMD & MAHNOMEN COUNTY WPA & Sand & Sandy Lake Native & 8 & 16 & 21 & 29 & 1 & 0 & 0 & 0 & 0.7 & 0 & 0.3 & 0 & 0.3 & 0 & 0.3 & 0.4 & 0.3 & 0 & 0.1 & 0.6 & & \\
\hline MORRIS WMD & BIG STONE COUNTY WPA & Hill & Hillman A & 9 & 17 & 22 & 30 & 1 & 0 & 0 & 0 & 0 & 1 & 0 & 0 & 0.1 & 0.2 & 0.6 & 0.1 & 0.2 & 0.2 & 0.1 & 0.5 & & \\
\hline MORRIS WMD & BIG STONE COUNTY WPA & Hill & Hillman B & 9 & 17 & 22 & 31 & 1 & 0 & 0 & 0 & 0 & 1 & 0 & 0 & 0.1 & 0.1 & 0.6 & 0.2 & 0.2 & 0.2 & 0.1 & 0.5 & & \\
\hline MORRIS WMD & BIG STONE COUNTY WPA & Hill & Hillman C & 9 & 17 & 22 & 32 & 1 & 0 & 0 & 0 & 0 & 1 & 0 & 0 & 0.1 & 0.1 & 0.6 & 0.2 & 0.2 & 0.2 & 0.1 & 0.5 & & \\
\hline MORRIS WMD & BIG STONE COUNTY WPA & Hill & Hillman D & 9 & 17 & 22 & 33 & 1 & 0 & 0 & 0 & 0 & 1 & 0 & 0 & 0.1 & 0.1 & 0.6 & 0.2 & 0.2 & 0.2 & 0.1 & 0.5 & & \\
\hline MORRIS WMD & LAC QUI PARLE COUNTY WPA & Flor & Florida Creek A & 9 & 18 & 23 & 34 & 1 & 0 & 0 & 0 & 0.5 & 0.1 & 0.2 & 0.2 & 0.3 & 0.1 & 0.5 & 0.1 & 0.5 & 0.1 & 0.1 & 0.3 & & \\
\hline MORRIS WMD & LAC QUI PARLE COUNTY WPA & Flor & Florida Creek B & 9 & 18 & 23 & 35 & 1 & 0 & 0 & 0 & 0.5 & 0.1 & 0.2 & 0.2 & 0.2 & 0.1 & 0.5 & 0.2 & 0.3 & 0.1 & 0.1 & 0.5 & & \\
\hline MORRIS WMD & LAC QUI PARLE COUNTY WPA & Flor & Florida Creek C & 9 & 18 & 23 & 36 & 1 & 0 & 0 & 0 & 0.5 & 0.1 & 0.2 & 0.2 & 0.2 & 0.1 & 0.5 & 0.2 & 0.3 & 0.1 & 0.1 & 0.5 & & \\
\hline MORRIS WMD & LAC QUI PARLE COUNTY WPA & Free & Freeland A & 9 & 18 & 24 & 37 & 1 & 0 & 0 & 0 & 0 & 1 & 0 & -7 & 0.2 & 0.2 & 0.5 & 0.1 & 0.2 & 0.2 & 0.1 & 0.5 & & \\
\hline MORRIS WMD & LAC QUI PARLE COUNTY WPA & Free & Freeland B & 9 & 18 & 24 & 38 & 1 & 0 & 0 & 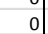 & 0 & 1 & 0 & 0 & 0.1 & 0.2 & 0.5 & 0.2 & 0.2 & 0.2 & 0.1 & 0.5 & & \\
\hline
\end{tabular}


Appendix 7a. South Dakota plant list.

[Includes plant codes, descriptions, group classifications, and classification into the four recognized vegetation types or components: native prairie (NP), smooth brome (SB), Kentucky bluegrass (KB), and remainder (RM) . Codes classified a NA are considered not applicable; stops along vegetation transects coded as NA were not included in the summed occurrences of the four components. The South Dakota plant list was used by cooperating stations that were located in South Dakota]

\begin{tabular}{|c|c|c|c|}
\hline Code & Description & Group & Component \\
\hline & 1 dense low shrub, other plants few or none & Shrub & RM \\
\hline 12 & 2 low shrub, remainder native grass and forb & Shrub & RM \\
\hline 13 & 3 low shrub, remainder $\mathrm{KY}$ bluegrass & Shrub & RM \\
\hline 14 & 4 low shrub, remainder brome or quackgrass & Shrub & RM \\
\hline 15 & 5 tall shrub, native & Shrub & RM \\
\hline 16 & 6 tall shrub, exotic & Shrub & RM \\
\hline 17 & 7 native trees (e.g. cottonwood, green ash, bur oak) & Tree & RM \\
\hline 18 & 8 non-native trees (e.g. Japanese elm, Russian olive) & Tree & RM \\
\hline 19 & low shrub, remainder crested & Shrub & RM \\
\hline 21 & 1 cool season grasses \& forbs A) green needle, B) western wheatgrass, C) porcupine grass & Native grass & NP \\
\hline 21.9 & 9 cool season $w /$ forbs & Native grass & NP \\
\hline 22 & 2 warm season grasses \& forbs A) big bluestem, B) switch, C) Indian, D) little bluestem & Native grass & NP \\
\hline 22.9 & warm season $\mathrm{w} /$ forbs & Native grass & NP \\
\hline 23 & 3 meadow (sedges, baltic rush, dock, smartweed, cordgrass, reedgrass, horsetail, foxtail barley, etc.) & Native sedges and rushes & NP \\
\hline 23.9 & meadow $w /$ forbs & Native sedges and rushes & NP \\
\hline 24 & 4 wetland; robust emergent vegetation or open water (cattail, river bulrush, bur-reed, Phragmites, manna grass) & Native wetland & NA \\
\hline 25 & 5 forb & Native forb & NP \\
\hline 31 & 1 Kentucky bluegrass dominant & KB & KB \\
\hline 31.9 & Kentucky bluegrass/forbs & KB & KB \\
\hline 41 & 1 smooth brome dominant & SM & SB \\
\hline 41.9 & Smooth brome w/ forbs & SB & SB \\
\hline 51 & 1 crested wheatgrass dominant & Invasive grass & RM \\
\hline 51.9 & 9 crested wheatgrass $w /$ forbs & Invasive grass & RM \\
\hline 52 & 2 quackgrass & Invasive grass & RM \\
\hline 52.9 & quackgrass $w /$ forbs & Invasive grass & RM \\
\hline 53 & 3 reed-canary grass & Invasive grass & RM \\
\hline 53.9 & 9 reed-canary grass $\mathrm{w} /$ forbs & Invasive grass & RM \\
\hline 61 & 1 Introduced wheatgrass & Invasive grass & RM \\
\hline 61.9 & Introduced wheatgrass $\mathrm{w} /$ forbs & Invasive grass & RM \\
\hline 62 & 2 other non-native grass - user defined (downy/Japanese brome, etc.) & Invasive grass & RM \\
\hline 62.9 & other non-native grass - user defined (downy/Japanese brome, etc.) w/ forbs & Invasive grass & RM \\
\hline 71 & 1 leafy spurge & Invasive forb & RM \\
\hline 72 & 2 canada thistle & Invasive forb & RM \\
\hline 73 & 3 sow thistle & Invasive forb & RM \\
\hline 74 & 4 wormwood & Invasive forb & RM \\
\hline 75 & 5 other weeds (kochia, ragweed, cocklebur, etc.) & Invasive forb & RM \\
\hline 76 & 5 other noxious weed (user-defined) & Invasive forb & RM \\
\hline 81 & 1 tall introduced legume (sweet clover or alfalfa) & Invasive forb & RM \\
\hline 83 & 3 cactus & Native & NP \\
\hline 84 & 4 clubmoss/Lichen & Native & NP \\
\hline 91 & 1 barren, unvegetated (bare soil, gopher mound) & Bare ground & NA \\
\hline 92 & 2 other (rock, manure, hole, ant hill) & Bare ground & NA \\
\hline
\end{tabular}


Appendix 7b. North Dakota plant list.

[Includes plant codes, descriptions, group classifications, and classification into the four recognized vegetation types or components: native prairie (NP), smooth brome (SB), Kentucky bluegrass (KB), and remainder (RM). Codes classified a NA are considered not applicable; stops along vegetation transects coded as NA were not included in the summed occurrences of the four components. The North Dakota plant list was used by cooperating stations that were located in North Dakota, Minnesota, and Montana]

\begin{tabular}{|c|c|}
\hline Code & Description \\
\hline 11 & snowberry dense; other plants few or none \\
\hline 12 & snowberry; remainder mostly NATIVE grass-forb types \\
\hline 13 & snowberry; remainder mostly Kentucky bluegrass \\
\hline 14 & snowberry; remainder mostly smooth brome (or quackgrass) \\
\hline 15 & silverberry \\
\hline 15.2 & silverberry; remainder mostly NATIVE grass-forb types \\
\hline 15.3 & silverberry; remainder mostly Kentucky bluegrass \\
\hline 15.4 & silverberry; remainder mostly smooth brome (or quackgrass) \\
\hline 15.5 & silverberry; remainder mostly crested wheatgrass \\
\hline 16 & snowberry; remainder mostly crested wheatgrass \\
\hline 18 & meadowsweet \\
\hline 18.2 & meadowsweet; remainder mostly NATIVE grass-forb types \\
\hline 18.3 & meadowsweet; remainder mostly Kentucy bluegrass \\
\hline 18.4 & meadowsweet; remainder mostly smooth brome (or quackgrass) \\
\hline 18.5 & meadowsweet; reaminder mostly crested wheatgrass \\
\hline 19 & other low shrub (user defined-add modifier) \\
\hline 21 & native shrub (chokecherry, buffaloberry, hawthorn, willow) \\
\hline 22 & shrub-stage aspen \\
\hline 23 & introduced shrub (caraganna, Russian olive) \\
\hline 31 & aspen \\
\hline 33 & shade-tolerant woodland tree (green ash, box elder, American elm) \\
\hline 34 & oak \\
\hline 35 & introduced tree (Siberian elm, Juniper, spruce) \\
\hline 41 & dry cool season (sedges, green needlegrass, needle-and-thread, wheatgrass spp., prairie junegrass, forbs) \\
\hline 42 & dry warm season (little bluestem, prairie sandreed, blue gramma, frobs) \\
\hline 43 & mesic cool-warm mix (big bluestem, switchgrass, porcupine grass, prairie dropseed, forbs) \\
\hline 46 & meadow (fowl bluegrass, foxtail barley, northern reedgrass, fine-stem sedge spp., baltic rush, cordgrass). \\
\hline 47 & wetland; robust emergent vegetation or open water (cattail, river bulrush, bur-reed, phragmites, manna grass \\
\hline 48 & clubmoss/lichen \\
\hline 49 & native forb \\
\hline 51 & Kentucky bluegrass $>95 \%$ (or $>50 \%$ if mixed with other non-natives) \\
\hline 52 & Kentucky bluegrass and NATIVE grass-forbs, $\mathrm{KY}$ bluegrass $50-95 \%$ \\
\hline 53 & NATIVE grass-forbs and Kentucky bluegrass, KY bluegrass $5-50 \%$ \\
\hline 61 & smooth brome $>95 \%$ (or $>50 \%$ if mixed with other non-natives) \\
\hline 62 & smooth brome and NATIVE grass-forbs, brome $50-95 \%$ \\
\hline 63 & NATIVE grass-forbs and smooth brome, brome $5-50 \%$ \\
\hline 71 & crested wheatgrass $>95 \%$ (or $>50 \%$ if mixed with other non-natives) \\
\hline 72 & crested wheatgrass and NATIVE grass-forbs, crested wheatgrass $50-95 \%$ \\
\hline 73 & NATIVE grass-forbs and crested wheatgrass, crested wheatgrass $5-50 \%$ \\
\hline 74 & quackgrass $>95 \%$ (or $>50 \%$ if mixed with other non-natives) \\
\hline 75 & quackgrass and NATIVE grass-forbs, quackgrass $50-95 \%$ \\
\hline 76 & NATIVE grass-forbs and quackgrass, quackgrass $5-50 \%$ \\
\hline $77 r$ & reed-canary grass \\
\hline 78 & tall, interm, or pub wheatgrass \\
\hline 79 & other introduced grass (user defined) \\
\hline 811 & leafy spurge \\
\hline 85 & Canada thistle \\
\hline $87 \sqrt{1}$ & wormwood \\
\hline $88 c$ & other introduced weeds (user-defined) \\
\hline $91 \mathrm{~b}$ & barren/unvegetated (e.g., rock, anthill, bare soil); dead vegetation \\
\hline $98 \mathrm{t}$ & tall introduced legume: sweet clover of alfalfa \\
\hline 990 & other-user defined \\
\hline
\end{tabular}

\begin{tabular}{|c|c|}
\hline Group & Component \\
\hline Shrub & RM \\
\hline Shrub & RM \\
\hline Shrub & RM \\
\hline Shrub & RM \\
\hline Shrub & RM \\
\hline Shrub & RM \\
\hline Shrub & RM \\
\hline Shrub & RM \\
\hline Shrub & RM \\
\hline Shrub & RM \\
\hline Shrub & RM \\
\hline Shrub & RM \\
\hline Shrub & RM \\
\hline Shrub & RM \\
\hline Shrub & RM \\
\hline Shrub & RM \\
\hline Shrub & RM \\
\hline Shrub & RM \\
\hline Shrub & RM \\
\hline Tree & RM \\
\hline Tree & RM \\
\hline Tree & RM \\
\hline Tree & RM \\
\hline Native grass & NP \\
\hline Native grass & NP \\
\hline Native grass & NP \\
\hline Native sedges and rushes & NP \\
\hline Native wetland & NA \\
\hline Native & NP \\
\hline Native forb & NP \\
\hline KB & KB \\
\hline KB & KB \\
\hline Native grass/forbs & NP \\
\hline SB & SB \\
\hline SB & SB \\
\hline Native grass/forbs & NP \\
\hline Invasive grass & RM \\
\hline Invasive grass & RM \\
\hline Native grass/forbs & NP \\
\hline Invasive grass & RM \\
\hline Invasive grass & RM \\
\hline Native grass/forbs & NP \\
\hline Invasive grass & RM \\
\hline Invasive grass & RM \\
\hline Invasive grass & RM \\
\hline Invasive forb & RM \\
\hline Invasive forb & RM \\
\hline Invasive forb & RM \\
\hline Invasive forb & RM \\
\hline Bare ground & NA \\
\hline Invasive forb & RM \\
\hline Other & RM \\
\hline
\end{tabular}


Appendix 8a. Summary of system state and management of mixed-grass units from cooperating stations from project inception (2009) through 2012.

[The summary includes the grass type, the name [complex and organization (org)] of cooperating stations and the enrolled units, management year, management applied, classification of the applied management, defoliation level, years since last defoliation, and vegetation state, in addition to the management recommendation for the following management year. $\mathrm{SB}=$ smooth brome, $\mathrm{CO}=$ co-dominant smooth brome and Kentucky bluegrass, $\mathrm{KB}=$ Kentucky bluegrass, $\mathrm{RM}=$ remainder, and $t=$ current management year. 2011 was the first management year for which management recommendations were provided to cooperators; thus, recommendations for the 2010 management year are documented as NA. Units that do not have data for all four years (2009-12) were either added to the project after its inception or were removed from the project after participating for a time. Following the summary are descriptions of the summary fields]

\begin{tabular}{|c|c|c|c|c|c|c|c|c|c|c|}
\hline $\begin{array}{l}\text { Grass } \\
\text { Type }\end{array}$ & Complex & Org & Unit & $\begin{array}{c}\text { Management } \\
\text { Year }\end{array}$ & $\begin{array}{c}\text { Management } \\
\text { Applied }\end{array}$ & $\begin{array}{c}\text { Management } \\
\text { Classified }\end{array}$ & $\begin{array}{c}\text { Defoliation } \\
\text { Level }\end{array}$ & $\begin{array}{l}\text { Years Since } \\
\text { Defoliation }\end{array}$ & $\begin{array}{l}\text { Vegetation } \\
\text { State }\end{array}$ & $\begin{array}{c}\text { Management } \\
\text { Recommendation } \\
\text { Year } t+1\end{array}$ \\
\hline Mixed & $\begin{array}{l}\text { ARROWWOOD } \\
\text { COMPLEX }\end{array}$ & $\begin{array}{c}\text { ARROWWOOD } \\
\text { NWR }\end{array}$ & G14 Pasture 1 & 2009 & Burn/Graze & NA & High & 1 & $\{30-45, \mathrm{CO}\}$ & NA \\
\hline Mixed & $\begin{array}{c}\text { ARROWWOOD } \\
\text { COMPLEX }\end{array}$ & $\begin{array}{c}\text { ARROWWOOD } \\
\text { NWR }\end{array}$ & G14 Pasture 1 & 2010 & Rest & Rest & High & $2-4$ & $\{30-45, \mathrm{CO}\}$ & Graze \\
\hline Mixed & $\begin{array}{c}\text { ARROWWOOD } \\
\text { COMPLEX }\end{array}$ & $\begin{array}{c}\text { ARROWWOOD } \\
\text { NWR }\end{array}$ & G14 Pasture 1 & 2011 & Rest & Rest & Med & $2-4$ & $\{0-30, \mathrm{CO}\}$ & Rest \\
\hline Mixed & $\begin{array}{c}\text { ARROWWOOD } \\
\text { COMPLEX }\end{array}$ & $\begin{array}{c}\text { ARROWWOOD } \\
\text { NWR }\end{array}$ & G14 Pasture 1 & 2012 & Burn & Burn & High & 1 & $\{45-60, \mathrm{CO}\}$ & Graze \\
\hline Mixed & $\begin{array}{l}\text { ARROWWOOD } \\
\text { COMPLEX }\end{array}$ & $\begin{array}{c}\text { ARROWWOOD } \\
\text { NWR }\end{array}$ & G14 Pasture 2 & 2009 & Burn & NA & High & 1 & $\{0-30, K B\}$ & NA \\
\hline Mixed & $\begin{array}{c}\text { ARROWWOOD } \\
\text { COMPLEX }\end{array}$ & $\begin{array}{c}\text { ARROWWOOD } \\
\text { NWR }\end{array}$ & G14 Pasture 2 & 2010 & Rest & Rest & High & $2-4$ & $\{0-30, \mathrm{CO}\}$ & Graze \\
\hline Mixed & $\begin{array}{l}\text { ARROWWOOD } \\
\text { COMPLEX }\end{array}$ & $\begin{array}{c}\text { ARROWWOOD } \\
\text { NWR }\end{array}$ & G14 Pasture 2 & 2011 & Rest & Rest & Med & $2-4$ & $\{0-30, \mathrm{CO}\}$ & Rest \\
\hline Mixed & $\begin{array}{l}\text { ARROWWOOD } \\
\text { COMPLEX }\end{array}$ & $\begin{array}{c}\text { ARROWWOOD } \\
\text { NWR }\end{array}$ & G14 Pasture 2 & 2012 & Burn & Burn & High & 1 & $\{60-100, R M\}$ & Rest \\
\hline Mixed & $\begin{array}{l}\text { ARROWWOOD } \\
\text { COMPLEX }\end{array}$ & $\begin{array}{c}\text { ARROWWOOD } \\
\text { NWR }\end{array}$ & G26 Paddock 1 & 2010 & Graze & Graze & High & 1 & $\{0-30, K B\}$ & Burn \\
\hline Mixed & $\begin{array}{l}\text { ARROWWOOD } \\
\text { COMPLEX }\end{array}$ & $\begin{array}{c}\text { ARROWWOOD } \\
\text { NWR }\end{array}$ & G26 Paddock 1 & 2011 & Graze & Graze & High & 1 & $\{0-30, K B\}$ & Graze \\
\hline
\end{tabular}




\begin{tabular}{|c|c|c|c|c|c|c|c|c|c|c|}
\hline $\begin{array}{l}\text { Grass } \\
\text { Type }\end{array}$ & Complex & Org & Unit & $\begin{array}{c}\text { Management } \\
\text { Year }\end{array}$ & $\begin{array}{c}\text { Management } \\
\text { Applied }\end{array}$ & $\begin{array}{c}\text { Management } \\
\text { Classified }\end{array}$ & $\begin{array}{l}\text { Defoliation } \\
\text { Level }\end{array}$ & $\begin{array}{l}\text { Years Since } \\
\text { Defoliation }\end{array}$ & $\begin{array}{c}\text { Vegetation } \\
\text { State }\end{array}$ & $\begin{array}{c}\text { Management } \\
\text { Recommendation } \\
\text { Year } t+1\end{array}$ \\
\hline Mixed & $\begin{array}{l}\text { ARROWWOOD } \\
\text { COMPLEX }\end{array}$ & $\begin{array}{c}\text { ARROWWOOD } \\
\text { NWR }\end{array}$ & G26 Paddock 1 & 2012 & Graze & Graze & High & 1 & $\{0-30, K B\}$ & Graze \\
\hline Mixed & $\begin{array}{l}\text { ARROWWOOD } \\
\text { COMPLEX }\end{array}$ & $\begin{array}{c}\text { ARROWWOOD } \\
\text { NWR }\end{array}$ & G26 Paddock 2 & 2010 & Graze & Graze & High & 1 & $\{0-30, K B\}$ & Burn \\
\hline Mixed & $\begin{array}{l}\text { ARROWWOOD } \\
\text { COMPLEX }\end{array}$ & $\begin{array}{c}\text { ARROWWOOD } \\
\text { NWR }\end{array}$ & G26 Paddock 2 & 2011 & Graze & Graze & High & 1 & $\{45-60, K B\}$ & Rest \\
\hline Mixed & $\begin{array}{l}\text { ARROWWOOD } \\
\text { COMPLEX }\end{array}$ & $\begin{array}{c}\text { ARROWWOOD } \\
\text { NWR }\end{array}$ & G26 Paddock 2 & 2012 & Burn/Graze & Burn/Graze & High & 1 & $\{60-100, K B\}$ & Graze \\
\hline Mixed & $\begin{array}{l}\text { ARROWWOOD } \\
\text { COMPLEX }\end{array}$ & $\begin{array}{c}\text { ARROWWOOD } \\
\text { NWR }\end{array}$ & G26 Paddock 3 & 2010 & Graze & Graze & High & 1 & $\{60-100, K B\}$ & Burn \\
\hline Mixed & $\begin{array}{l}\text { ARROWWOOD } \\
\text { COMPLEX }\end{array}$ & $\begin{array}{c}\text { ARROWWOOD } \\
\text { NWR }\end{array}$ & G26 Paddock 3 & 2011 & Graze & Graze & High & 1 & $\{0-30, \mathrm{CO}\}$ & Graze \\
\hline Mixed & $\begin{array}{l}\text { ARROWWOOD } \\
\text { COMPLEX }\end{array}$ & $\begin{array}{c}\text { ARROWWOOD } \\
\text { NWR }\end{array}$ & G26 Paddock 3 & 2012 & Graze & Graze & High & 1 & $\{0-30, K B\}$ & Graze \\
\hline Mixed & $\begin{array}{l}\text { ARROWWOOD } \\
\text { COMPLEX }\end{array}$ & $\begin{array}{c}\text { ARROWWOOD } \\
\text { NWR }\end{array}$ & G26 Paddock 4 & 2010 & Graze & Graze & High & 1 & $\{0-30, K B\}$ & Burn \\
\hline Mixed & $\begin{array}{l}\text { ARROWWOOD } \\
\text { COMPLEX }\end{array}$ & $\begin{array}{c}\text { ARROWWOOD } \\
\text { NWR }\end{array}$ & G26 Paddock 4 & 2011 & Graze & Graze & High & 1 & $\{0-30, K B\}$ & Graze \\
\hline Mixed & $\begin{array}{l}\text { ARROWWOOD } \\
\text { COMPLEX }\end{array}$ & $\begin{array}{c}\text { ARROWWOOD } \\
\text { NWR }\end{array}$ & G26 Paddock 4 & 2012 & Graze & Graze & High & 1 & $\{0-30, K B\}$ & Graze \\
\hline Mixed & $\begin{array}{l}\text { ARROWWOOD } \\
\text { COMPLEX }\end{array}$ & $\begin{array}{c}\text { ARROWWOOD } \\
\text { NWR }\end{array}$ & G28 & 2009 & Rest & NA & Low & $2-4$ & $\{0-30, K B\}$ & NA \\
\hline Mixed & $\begin{array}{l}\text { ARROWWOOD } \\
\text { COMPLEX }\end{array}$ & $\begin{array}{c}\text { ARROWWOOD } \\
\text { NWR }\end{array}$ & G28 & 2010 & Rest & Rest & Low & $2-4$ & $\{0-30, K B\}$ & Burn \\
\hline Mixed & $\begin{array}{l}\text { ARROWWOOD } \\
\text { COMPLEX }\end{array}$ & $\begin{array}{c}\text { ARROWWOOD } \\
\text { NWR }\end{array}$ & $\mathrm{G} 28$ & 2011 & Burn & Burn & Low & 1 & $\{0-30, K B\}$ & Burn/Graze \\
\hline Mixed & $\begin{array}{l}\text { ARROWWOOD } \\
\text { COMPLEX }\end{array}$ & $\begin{array}{c}\text { ARROWWOOD } \\
\text { NWR }\end{array}$ & G28 & 2012 & Rest & Rest & Low & $2-4$ & $\{30-45, K B\}$ & Burn \\
\hline Mixed & $\begin{array}{l}\text { ARROWWOOD } \\
\text { COMPLEX }\end{array}$ & EDDY COUNTY WPA & Haven Paddock 4 & 2010 & Graze & Graze & High & 1 & $\{60-100, S B\}$ & Graze \\
\hline Mixed & $\begin{array}{l}\text { ARROWWOOD } \\
\text { COMPLEX }\end{array}$ & EDDY COUNTY WPA & Haven Paddock 4 & 2011 & Graze & Graze & High & 1 & $\{60-100, \mathrm{RM}\}$ & Rest \\
\hline
\end{tabular}




\begin{tabular}{|c|c|c|c|c|c|c|c|c|c|c|}
\hline $\begin{array}{l}\text { Grass } \\
\text { Type }\end{array}$ & Complex & Org & Unit & $\begin{array}{c}\text { Management } \\
\text { Year }\end{array}$ & $\begin{array}{c}\text { Management } \\
\text { Applied }\end{array}$ & $\begin{array}{l}\text { Management } \\
\text { Classified }\end{array}$ & $\begin{array}{l}\text { Defoliation } \\
\text { Level }\end{array}$ & $\begin{array}{l}\text { Years Since } \\
\text { Defoliation }\end{array}$ & $\begin{array}{c}\text { Vegetation } \\
\text { State }\end{array}$ & $\begin{array}{c}\text { Management } \\
\text { Recommendation } \\
\text { Year } t+1 \\
\end{array}$ \\
\hline Mixed & $\begin{array}{l}\text { ARROWWOOD } \\
\text { COMPLEX }\end{array}$ & EDDY COUNTY WPA & Haven Paddock 4 & 2012 & Burn/Graze & Burn/Graze & High & 1 & $\{60-100, \mathrm{CO}\}$ & Rest \\
\hline Mixed & $\begin{array}{l}\text { ARROWWOOD } \\
\text { COMPLEX }\end{array}$ & EDDY COUNTY WPA & Haven Paddock 5 & 2010 & Graze & Graze & High & 1 & $\{45-60, K B\}$ & Burn \\
\hline Mixed & $\begin{array}{l}\text { ARROWWOOD } \\
\text { COMPLEX }\end{array}$ & EDDY COUNTY WPA & Haven Paddock 5 & 2011 & Graze & Graze & High & 1 & $\{45-60, \mathrm{CO}\}$ & Graze \\
\hline Mixed & $\begin{array}{l}\text { ARROWWOOD } \\
\text { COMPLEX }\end{array}$ & EDDY COUNTY WPA & Haven Paddock 5 & 2012 & Burn/Graze & Burn/Graze & High & 1 & $\{60-100, K B\}$ & Graze \\
\hline Mixed & $\begin{array}{l}\text { ARROWWOOD } \\
\text { COMPLEX }\end{array}$ & $\begin{array}{c}\text { FOSTER COUNTY } \\
\text { WPA }\end{array}$ & $\begin{array}{l}\text { Topp East } \\
\text { Paddock }\end{array}$ & 2009 & Graze & NA & High & 1 & $\{30-45, K B\}$ & NA \\
\hline Mixed & $\begin{array}{l}\text { ARROWWOOD } \\
\text { COMPLEX }\end{array}$ & $\begin{array}{c}\text { FOSTER COUNTY } \\
\text { WPA }\end{array}$ & $\begin{array}{l}\text { Topp East } \\
\text { Paddock }\end{array}$ & 2010 & Graze & Graze & High & 1 & $\{0-30, K B\}$ & Burn \\
\hline Mixed & $\begin{array}{l}\text { ARROWWOOD } \\
\text { COMPLEX }\end{array}$ & $\begin{array}{c}\text { FOSTER COUNTY } \\
\text { WPA }\end{array}$ & $\begin{array}{l}\text { Topp East } \\
\text { Paddock }\end{array}$ & 2011 & Graze & Graze & High & 1 & $\{30-45, K B\}$ & Burn/Graze \\
\hline Mixed & $\begin{array}{l}\text { ARROWWOOD } \\
\text { COMPLEX }\end{array}$ & $\begin{array}{l}\text { FOSTER COUNTY } \\
\text { WPA }\end{array}$ & $\begin{array}{l}\text { Topp East } \\
\text { Paddock }\end{array}$ & 2012 & Burn/Graze & Burn/Graze & High & 1 & $\{60-100, K B\}$ & Graze \\
\hline Mixed & $\begin{array}{l}\text { ARROWWOOD } \\
\text { COMPLEX }\end{array}$ & $\begin{array}{c}\text { FOSTER COUNTY } \\
\text { WPA }\end{array}$ & $\begin{array}{l}\text { Topp West } \\
\text { Paddock }\end{array}$ & 2009 & Graze & NA & High & 1 & $\{45-60, K B\}$ & NA \\
\hline Mixed & $\begin{array}{l}\text { ARROWWOOD } \\
\text { COMPLEX }\end{array}$ & $\begin{array}{c}\text { FOSTER COUNTY } \\
\text { WPA }\end{array}$ & $\begin{array}{l}\text { Topp West } \\
\text { Paddock }\end{array}$ & 2010 & Graze & Graze & High & 1 & $\{60-100, K B\}$ & Burn \\
\hline Mixed & $\begin{array}{l}\text { ARROWWOOD } \\
\text { COMPLEX }\end{array}$ & $\begin{array}{c}\text { FOSTER COUNTY } \\
\text { WPA }\end{array}$ & $\begin{array}{l}\text { Topp West } \\
\text { Paddock }\end{array}$ & 2011 & Rest & Rest & High & $2-4$ & $\{45-60, K B\}$ & Rest \\
\hline Mixed & $\begin{array}{l}\text { ARROWWOOD } \\
\text { COMPLEX }\end{array}$ & $\begin{array}{c}\text { FOSTER COUNTY } \\
\text { WPA }\end{array}$ & $\begin{array}{l}\text { Topp West } \\
\text { Paddock }\end{array}$ & 2012 & Burn/Graze & Burn/Graze & High & 1 & $\{45-60, K B\}$ & Rest \\
\hline Mixed & $\begin{array}{l}\text { ARROWWOOD } \\
\text { COMPLEX }\end{array}$ & $\begin{array}{c}\text { GRIGGS COUNTY } \\
\text { WPA }\end{array}$ & Wogsland & 2009 & Rest & NA & Low & $5+$ & $\{45-60, \mathrm{RM}\}$ & NA \\
\hline Mixed & $\begin{array}{l}\text { ARROWWOOD } \\
\text { COMPLEX }\end{array}$ & $\begin{array}{c}\text { GRIGGS COUNTY } \\
\text { WPA }\end{array}$ & Wogsland & 2010 & Rest & Rest & Low & $5+$ & $\{45-60, S B\}$ & Graze \\
\hline Mixed & $\begin{array}{l}\text { ARROWWOOD } \\
\text { COMPLEX }\end{array}$ & $\begin{array}{c}\text { GRIGGS COUNTY } \\
\text { WPA }\end{array}$ & Wogsland & 2011 & Rest & Rest & Low & $5+$ & $\{45-60, \mathrm{CO}\}$ & Burn/Graze \\
\hline Mixed & $\begin{array}{l}\text { ARROWWOOD } \\
\text { COMPLEX }\end{array}$ & $\begin{array}{c}\text { GRIGGS COUNTY } \\
\text { WPA }\end{array}$ & Wogsland & 2012 & Rest & Rest & Low & $5+$ & $\{60-100, \mathrm{CO}\}$ & Burn \\
\hline
\end{tabular}




\begin{tabular}{|c|c|c|c|c|c|c|c|c|c|c|}
\hline $\begin{array}{l}\text { Grass } \\
\text { Type }\end{array}$ & Complex & Org & Unit & $\begin{array}{c}\text { Management } \\
\text { Year }\end{array}$ & $\begin{array}{c}\text { Management } \\
\text { Applied }\end{array}$ & $\begin{array}{l}\text { Management } \\
\text { Classified }\end{array}$ & $\begin{array}{l}\text { Defoliation } \\
\text { Level }\end{array}$ & $\begin{array}{l}\text { Years Since } \\
\text { Defoliation }\end{array}$ & $\begin{array}{l}\text { Vegetation } \\
\text { State }\end{array}$ & $\begin{array}{c}\text { Management } \\
\text { Recommendation } \\
\text { Year } t+1 \\
\end{array}$ \\
\hline Mixed & $\begin{array}{l}\text { ARROWWOOD } \\
\text { COMPLEX }\end{array}$ & $\begin{array}{c}\text { STUTSMAN COUNTY } \\
\text { WPA }\end{array}$ & Odegaard & 2009 & Burn & NA & Med & 1 & $\{30-45, S B\}$ & NA \\
\hline Mixed & $\begin{array}{l}\text { ARROWWOOD } \\
\text { COMPLEX }\end{array}$ & $\begin{array}{c}\text { STUTSMAN COUNTY } \\
\text { WPA }\end{array}$ & Odegaard & 2010 & Rest & Rest & Med & $2-4$ & $\{30-45, S B\}$ & Graze \\
\hline Mixed & $\begin{array}{l}\text { ARROWWOOD } \\
\text { COMPLEX }\end{array}$ & $\begin{array}{c}\text { STUTSMAN COUNTY } \\
\text { WPA }\end{array}$ & Odegaard & 2011 & Rest & Rest & Med & $2-4$ & $\{0-30, S B\}$ & Graze \\
\hline Mixed & $\begin{array}{l}\text { ARROWWOOD } \\
\text { COMPLEX }\end{array}$ & $\begin{array}{c}\text { STUTSMAN COUNTY } \\
\text { WPA }\end{array}$ & Odegaard & 2012 & Rest & Rest & Low & $2-4$ & $\{0-30, S B\}$ & Graze \\
\hline Mixed & $\begin{array}{l}\text { ARROWWOOD } \\
\text { COMPLEX }\end{array}$ & $\begin{array}{c}\text { STUTSMAN COUNTY } \\
\text { WPA }\end{array}$ & Winberg Unit 1 & 2009 & Graze & NA & Med & 1 & $\{45-60, K B\}$ & NA \\
\hline Mixed & $\begin{array}{l}\text { ARROWWOOD } \\
\text { COMPLEX }\end{array}$ & $\begin{array}{c}\text { STUTSMAN COUNTY } \\
\text { WPA }\end{array}$ & Winberg Unit 1 & 2010 & Rest & Rest & Low & $2-4$ & $\{45-60, K B\}$ & Burn \\
\hline Mixed & $\begin{array}{l}\text { ARROWWOOD } \\
\text { COMPLEX }\end{array}$ & $\begin{array}{c}\text { STUTSMAN COUNTY } \\
\text { WPA }\end{array}$ & Winberg Unit 1 & 2011 & Rest & Rest & Low & $2-4$ & $\{45-60, K B\}$ & Burn \\
\hline Mixed & $\begin{array}{l}\text { ARROWWOOD } \\
\text { COMPLEX }\end{array}$ & $\begin{array}{c}\text { STUTSMAN COUNTY } \\
\text { WPA }\end{array}$ & Winberg Unit 1 & 2012 & Burn & Burn & Med & 1 & $\{60-100, \mathrm{CO}\}$ & Rest \\
\hline Mixed & $\begin{array}{l}\text { ARROWWOOD } \\
\text { COMPLEX }\end{array}$ & $\begin{array}{c}\text { STUTSMAN COUNTY } \\
\text { WPA }\end{array}$ & $\begin{array}{l}\text { Woodworth } \\
\text { Station Unit } 7\end{array}$ & 2009 & Burn & NA & Low & 1 & $\{30-45, S B\}$ & NA \\
\hline Mixed & $\begin{array}{l}\text { ARROWWOOD } \\
\text { COMPLEX }\end{array}$ & $\begin{array}{c}\text { STUTSMAN COUNTY } \\
\text { WPA }\end{array}$ & $\begin{array}{l}\text { Woodworth } \\
\text { Station Unit } 7\end{array}$ & 2010 & hay & NA & Low & 1 & $\{0-30, S B\}$ & Graze \\
\hline Mixed & $\begin{array}{l}\text { ARROWWOOD } \\
\text { COMPLEX }\end{array}$ & $\begin{array}{c}\text { STUTSMAN COUNTY } \\
\text { WPA }\end{array}$ & $\begin{array}{l}\text { Woodworth } \\
\text { Station Unit } 7\end{array}$ & 2011 & Graze & Graze & Med & 1 & $\{0-30, \mathrm{CO}\}$ & Rest \\
\hline Mixed & $\begin{array}{l}\text { ARROWWOOD } \\
\text { COMPLEX }\end{array}$ & $\begin{array}{c}\text { STUTSMAN COUNTY } \\
\text { WPA }\end{array}$ & $\begin{array}{l}\text { Woodworth } \\
\text { Station Unit } 7\end{array}$ & 2012 & Graze & Graze & Med & 1 & $\{0-30, S B\}$ & Graze \\
\hline Mixed & $\begin{array}{l}\text { ARROWWOOD } \\
\text { COMPLEX }\end{array}$ & $\begin{array}{l}\text { WELLS COUNTY } \\
\text { WPA }\end{array}$ & Frederick & 2009 & Rest & NA & Low & $5+$ & $\{30-45, \mathrm{CO}\}$ & NA \\
\hline Mixed & $\begin{array}{l}\text { ARROWWOOD } \\
\text { COMPLEX }\end{array}$ & $\begin{array}{l}\text { WELLS COUNTY } \\
\text { WPA }\end{array}$ & Frederick & 2010 & Rest & Rest & Low & $5+$ & $\{45-60, S B\}$ & Graze \\
\hline Mixed & $\begin{array}{l}\text { ARROWWOOD } \\
\text { COMPLEX }\end{array}$ & $\begin{array}{c}\text { WELLS COUNTY } \\
\text { WPA }\end{array}$ & Frederick & 2011 & Rest & Rest & Low & $5+$ & $\{0-30, S B\}$ & Burn \\
\hline Mixed & $\begin{array}{l}\text { ARROWWOOD } \\
\text { COMPLEX }\end{array}$ & $\begin{array}{c}\text { WELLS COUNTY } \\
\text { WPA }\end{array}$ & Frederick & 2012 & Rest & Rest & Low & $5+$ & $\{0-30, \mathrm{CO}\}$ & Graze \\
\hline
\end{tabular}




\begin{tabular}{|c|c|c|c|c|c|c|c|c|c|c|}
\hline $\begin{array}{l}\text { Grass } \\
\text { Type }\end{array}$ & Complex & Org & Unit & $\begin{array}{c}\text { Management } \\
\text { Year }\end{array}$ & $\begin{array}{c}\text { Management } \\
\text { Applied }\end{array}$ & $\begin{array}{l}\text { Management } \\
\text { Classified }\end{array}$ & $\begin{array}{l}\text { Defoliation } \\
\text { Level }\end{array}$ & $\begin{array}{l}\text { Years Since } \\
\text { Defoliation }\end{array}$ & $\begin{array}{l}\text { Vegetation } \\
\text { State }\end{array}$ & $\begin{array}{c}\text { Management } \\
\text { Recommendation } \\
\text { Year } t+1\end{array}$ \\
\hline Mixed & AUDUBON COMPLEX & $\begin{array}{l}\text { MCLEAN COUNTY } \\
\text { WPA }\end{array}$ & $\begin{array}{c}\text { Koenig Section } \\
\text { Line Slough Unit }\end{array}$ & 2009 & Burn & NA & Med & 1 & $\{0-30, K B\}$ & NA \\
\hline Mixed & AUDUBON COMPLEX & $\begin{array}{l}\text { MCLEAN COUNTY } \\
\text { WPA }\end{array}$ & $\begin{array}{c}\text { Koenig Section } \\
\text { Line Slough Unit }\end{array}$ & 2010 & Graze & Graze & Med & 1 & $\{30-45, K B\}$ & Burn \\
\hline Mixed & AUDUBON COMPLEX & $\begin{array}{l}\text { MCLEAN COUNTY } \\
\text { WPA }\end{array}$ & $\begin{array}{c}\text { Koenig Section } \\
\text { Line Slough Unit }\end{array}$ & 2011 & Burn & Burn & High & 1 & $\{0-30, K B\}$ & Graze \\
\hline Mixed & AUDUBON COMPLEX & $\begin{array}{l}\text { MCLEAN COUNTY } \\
\text { WPA }\end{array}$ & $\begin{array}{c}\text { Koenig Section } \\
\text { Line Slough Unit }\end{array}$ & 2012 & Graze & Graze & High & 1 & $\{30-45, K B\}$ & Burn/Graze \\
\hline Mixed & AUDUBON COMPLEX & $\begin{array}{l}\text { MCLEAN COUNTY } \\
\text { WPA }\end{array}$ & Otis Unit 5A & 2009 & Rest & NA & High & $2-4$ & $\{0-30, R M\}$ & NA \\
\hline Mixed & AUDUBON COMPLEX & $\begin{array}{l}\text { MCLEAN COUNTY } \\
\text { WPA }\end{array}$ & Otis Unit 5A & 2010 & Graze & Graze & High & 1 & $\{45-60, S B\}$ & Graze \\
\hline Mixed & AUDUBON COMPLEX & $\begin{array}{c}\text { MCLEAN COUNTY } \\
\text { WPA }\end{array}$ & Otis Unit 5A & 2011 & Graze & Graze & High & 1 & $\{0-30, R M\}$ & Rest \\
\hline Mixed & AUDUBON COMPLEX & $\begin{array}{c}\text { MCLEAN COUNTY } \\
\text { WPA }\end{array}$ & Otis Unit 5A & 2012 & Rest & Rest & High & $2-4$ & $\{0-30, K B\}$ & Graze \\
\hline Mixed & AUDUBON COMPLEX & $\begin{array}{l}\text { MCLEAN COUNTY } \\
\text { WPA }\end{array}$ & Otis Unit 5B & 2009 & Rest & NA & High & $2-4$ & $\{0-30, K B\}$ & NA \\
\hline Mixed & AUDUBON COMPLEX & $\begin{array}{c}\text { MCLEAN COUNTY } \\
\text { WPA }\end{array}$ & Otis Unit 5B & 2010 & Graze & Graze & High & 1 & $\{30-45, K B\}$ & Burn \\
\hline Mixed & AUDUBON COMPLEX & $\begin{array}{l}\text { MCLEAN COUNTY } \\
\text { WPA }\end{array}$ & Otis Unit 5B & 2011 & Burn & Burn & High & 1 & $\{45-60, R M\}$ & Rest \\
\hline Mixed & AUDUBON COMPLEX & $\begin{array}{l}\text { MCLEAN COUNTY } \\
\text { WPA }\end{array}$ & Otis Unit 5B & 2012 & Rest & Rest & High & $2-4$ & $\{45-60, \mathrm{RM}\}$ & Graze \\
\hline Mixed & AUDUBON COMPLEX & $\begin{array}{c}\text { MCLEAN COUNTY } \\
\text { WPA }\end{array}$ & Otis Unit 8N & 2009 & Graze & NA & High & 1 & $\{0-30, \mathrm{RM}\}$ & NA \\
\hline Mixed & AUDUBON COMPLEX & $\begin{array}{l}\text { MCLEAN COUNTY } \\
\text { WPA }\end{array}$ & Otis Unit 8N & 2010 & Graze & Graze & High & 1 & $\{0-30, K B\}$ & Burn \\
\hline Mixed & AUDUBON COMPLEX & $\begin{array}{l}\text { MCLEAN COUNTY } \\
\text { WPA }\end{array}$ & Otis Unit 8N & 2011 & Burn & Burn & High & 1 & $\{0-30, K B\}$ & Graze \\
\hline Mixed & AUDUBON COMPLEX & $\begin{array}{c}\text { MCLEAN COUNTY } \\
\text { WPA }\end{array}$ & Otis Unit 8N & 2012 & Graze & Graze & High & 1 & $\{30-45, \mathrm{RM}\}$ & Graze \\
\hline
\end{tabular}




\begin{tabular}{|c|c|c|c|c|c|c|c|c|c|c|}
\hline $\begin{array}{l}\text { Grass } \\
\text { Type }\end{array}$ & Complex & Org & Unit & $\begin{array}{c}\text { Management } \\
\text { Year }\end{array}$ & $\begin{array}{c}\text { Management } \\
\text { Applied }\end{array}$ & $\begin{array}{l}\text { Management } \\
\text { Classified }\end{array}$ & $\begin{array}{l}\text { Defoliation } \\
\text { Level }\end{array}$ & $\begin{array}{l}\text { Years Since } \\
\text { Defoliation }\end{array}$ & $\begin{array}{l}\text { Vegetation } \\
\text { State }\end{array}$ & $\begin{array}{c}\text { Management } \\
\text { Recommendation } \\
\text { Year } t+1\end{array}$ \\
\hline Mixed & AUDUBON COMPLEX & $\begin{array}{c}\text { MCLEAN COUNTY } \\
\text { WPA }\end{array}$ & Otis Unit 8S & 2009 & Rest & NA & High & $2-4$ & $\{0-30, \mathrm{RM}\}$ & NA \\
\hline Mixed & AUDUBON COMPLEX & $\begin{array}{c}\text { MCLEAN COUNTY } \\
\text { WPA }\end{array}$ & Otis Unit 8S & 2010 & Graze & Graze & High & 1 & $\{0-30, \mathrm{CO}\}$ & Graze \\
\hline Mixed & AUDUBON COMPLEX & $\begin{array}{l}\text { MCLEAN COUNTY } \\
\text { WPA }\end{array}$ & Otis Unit 8S & 2011 & Burn & Burn & High & 1 & $\{45-60, \mathrm{CO}\}$ & Graze \\
\hline Mixed & AUDUBON COMPLEX & $\begin{array}{l}\text { MCLEAN COUNTY } \\
\text { WPA }\end{array}$ & Otis Unit $8 \mathrm{~S}$ & 2012 & Graze & Graze & High & 1 & $\{30-45, \mathrm{RM}\}$ & Graze \\
\hline Mixed & AUDUBON COMPLEX & $\begin{array}{c}\text { SHERIDAN COUNTY } \\
\text { WPA }\end{array}$ & Lasher Unit A & 2009 & Graze & NA & High & 1 & $\{60-100, K B\}$ & NA \\
\hline Mixed & AUDUBON COMPLEX & $\begin{array}{c}\text { SHERIDAN COUNTY } \\
\text { WPA }\end{array}$ & Lasher Unit A & 2010 & Rest & Rest & Med & $2-4$ & $\{0-30, K B\}$ & Burn \\
\hline Mixed & AUDUBON COMPLEX & $\begin{array}{c}\text { SHERIDAN COUNTY } \\
\text { WPA }\end{array}$ & Lasher Unit A & 2011 & Burn & Burn & Med & 1 & $\{30-45, K B\}$ & Burn \\
\hline Mixed & AUDUBON COMPLEX & $\begin{array}{c}\text { SHERIDAN COUNTY } \\
\text { WPA }\end{array}$ & Lasher Unit A & 2012 & Burn & Burn & High & 1 & $\{60-100, K B\}$ & Graze \\
\hline Mixed & AUDUBON COMPLEX & $\begin{array}{c}\text { SHERIDAN COUNTY } \\
\text { WPA }\end{array}$ & Lasher Unit B & 2009 & Graze & NA & High & 1 & $\{45-60, K B\}$ & NA \\
\hline Mixed & AUDUBON COMPLEX & $\begin{array}{c}\text { SHERIDAN COUNTY } \\
\text { WPA }\end{array}$ & Lasher Unit B & 2010 & Rest & Rest & Med & $2-4$ & $\{0-30, K B\}$ & Burn \\
\hline Mixed & AUDUBON COMPLEX & $\begin{array}{c}\text { SHERIDAN COUNTY } \\
\text { WPA }\end{array}$ & Lasher Unit B & 2011 & Burn & Burn & Med & 1 & $\{30-45, K B\}$ & Burn \\
\hline Mixed & AUDUBON COMPLEX & $\begin{array}{c}\text { SHERIDAN COUNTY } \\
\text { WPA }\end{array}$ & Lasher Unit B & 2012 & Burn & Burn & High & 1 & $\{0-30, K B\}$ & Graze \\
\hline Mixed & AUDUBON COMPLEX & $\begin{array}{l}\text { WARD COUNTY } \\
\text { WPA }\end{array}$ & Peterson NE & 2009 & Graze & NA & High & 1 & $\{45-60, \mathrm{CO}\}$ & NA \\
\hline Mixed & AUDUBON COMPLEX & $\begin{array}{l}\text { WARD COUNTY } \\
\text { WPA }\end{array}$ & Peterson NE & 2010 & Rest & Rest & High & $2-4$ & $\{30-45, K B\}$ & Burn \\
\hline Mixed & AUDUBON COMPLEX & $\begin{array}{l}\text { WARD COUNTY } \\
\text { WPA }\end{array}$ & Peterson NE & 2011 & Graze & Graze & High & 1 & $\{60-100, K B\}$ & Graze \\
\hline Mixed & AUDUBON COMPLEX & $\begin{array}{l}\text { WARD COUNTY } \\
\text { WPA }\end{array}$ & Peterson NE & 2012 & Graze & Graze & High & 1 & $\{45-60, R M\}$ & Rest \\
\hline
\end{tabular}




\begin{tabular}{|c|c|c|c|c|c|c|c|c|c|c|}
\hline $\begin{array}{l}\text { Grass } \\
\text { Type }\end{array}$ & Complex & Org & Unit & $\begin{array}{c}\text { Management } \\
\text { Year }\end{array}$ & $\begin{array}{c}\text { Management } \\
\text { Applied }\end{array}$ & $\begin{array}{c}\text { Management } \\
\text { Classified }\end{array}$ & $\begin{array}{l}\text { Defoliation } \\
\text { Level }\end{array}$ & $\begin{array}{l}\text { Years Since } \\
\text { Defoliation }\end{array}$ & $\begin{array}{c}\text { Vegetation } \\
\text { State }\end{array}$ & $\begin{array}{c}\text { Management } \\
\text { Recommendation } \\
\text { Year } t+1\end{array}$ \\
\hline Mixed & AUDUBON COMPLEX & $\begin{array}{l}\text { WARD COUNTY } \\
\text { WPA }\end{array}$ & Peterson NW & 2009 & Graze & NA & High & 1 & $\{30-45, K B\}$ & NA \\
\hline Mixed & AUDUBON COMPLEX & $\begin{array}{c}\text { WARD COUNTY } \\
\text { WPA }\end{array}$ & Peterson NW & 2010 & Rest & Rest & High & $2-4$ & $\{30-45, \mathrm{CO}\}$ & Graze \\
\hline Mixed & AUDUBON COMPLEX & $\begin{array}{l}\text { WARD COUNTY } \\
\text { WPA }\end{array}$ & Peterson NW & 2011 & Graze & Graze & High & 1 & $\{30-45, \mathrm{RM}\}$ & Graze \\
\hline Mixed & AUDUBON COMPLEX & $\begin{array}{l}\text { WARD COUNTY } \\
\text { WPA }\end{array}$ & Peterson NW & 2012 & Graze & Graze & High & 1 & $\{0-30, \mathrm{CO}\}$ & Graze \\
\hline Mixed & AUDUBON COMPLEX & $\begin{array}{l}\text { WARD COUNTY } \\
\text { WPA }\end{array}$ & Peterson SE & 2009 & Rest & NA & High & $2-4$ & $\{0-30, \mathrm{CO}\}$ & NA \\
\hline Mixed & AUDUBON COMPLEX & $\begin{array}{c}\text { WARD COUNTY } \\
\text { WPA }\end{array}$ & Peterson SE & 2010 & Graze & Graze & High & 1 & $\{0-30, \mathrm{CO}\}$ & Graze \\
\hline Mixed & AUDUBON COMPLEX & $\begin{array}{c}\text { WARD COUNTY } \\
\text { WPA }\end{array}$ & Peterson SE & 2011 & Rest & Rest & High & $2-4$ & $\{30-45, \mathrm{CO}\}$ & Burn/Graze \\
\hline Mixed & AUDUBON COMPLEX & $\begin{array}{c}\text { WARD COUNTY } \\
\text { WPA }\end{array}$ & Peterson SE & 2012 & Burn/Graze & Burn/Graze & High & 1 & $\{45-60, S B\}$ & Rest \\
\hline Mixed & AUDUBON COMPLEX & $\begin{array}{l}\text { WARD COUNTY } \\
\text { WPA }\end{array}$ & Peterson SW & 2009 & Rest & NA & High & $2-4$ & $\{45-60, \mathrm{CO}\}$ & NA \\
\hline Mixed & AUDUBON COMPLEX & $\begin{array}{l}\text { WARD COUNTY } \\
\text { WPA }\end{array}$ & Peterson SW & 2010 & Graze & Graze & High & 1 & $\{45-60, \mathrm{CO}\}$ & Burn \\
\hline Mixed & AUDUBON COMPLEX & $\begin{array}{l}\text { WARD COUNTY } \\
\text { WPA }\end{array}$ & Peterson SW & 2011 & Rest & Rest & High & $2-4$ & $\{0-30, K B\}$ & Graze \\
\hline Mixed & AUDUBON COMPLEX & $\begin{array}{l}\text { WARD COUNTY } \\
\text { WPA }\end{array}$ & Peterson SW & 2012 & Burn/Graze & Burn/Graze & High & 1 & $\{60-100, K B\}$ & Graze \\
\hline Mixed & BENTON LAKE WMD & $\begin{array}{c}\text { TOOLE COUNTY } \\
\text { WPA }\end{array}$ & Ehli & 2009 & Rest & NA & Low & $5+$ & $\{0-30, K B\}$ & NA \\
\hline Mixed & BENTON LAKE WMD & $\begin{array}{c}\text { TOOLE COUNTY } \\
\text { WPA }\end{array}$ & Ehli & 2010 & Burn & Burn & Low & 1 & $\{30-45, K B\}$ & Burn \\
\hline Mixed & BENTON LAKE WMD & $\begin{array}{c}\text { TOOLE COUNTY } \\
\text { WPA }\end{array}$ & Ehli & 2011 & Rest & Rest & Low & $2-4$ & $\{30-45, K B\}$ & Burn \\
\hline Mixed & BENTON LAKE WMD & $\begin{array}{l}\text { TOOLE COUNTY } \\
\text { WPA }\end{array}$ & Ehli & 2012 & Rest & Rest & Low & $2-4$ & $\{0-30, K B\}$ & Graze \\
\hline
\end{tabular}




\begin{tabular}{|c|c|c|c|c|c|c|c|c|c|c|}
\hline $\begin{array}{l}\text { Grass } \\
\text { Type }\end{array}$ & Complex & Org & Unit & $\begin{array}{c}\text { Management } \\
\text { Year }\end{array}$ & $\begin{array}{c}\text { Management } \\
\text { Applied }\end{array}$ & $\begin{array}{l}\text { Management } \\
\text { Classified }\end{array}$ & $\begin{array}{l}\text { Defoliation } \\
\text { Level }\end{array}$ & $\begin{array}{l}\text { Years Since } \\
\text { Defoliation }\end{array}$ & $\begin{array}{l}\text { Vegetation } \\
\text { State }\end{array}$ & $\begin{array}{c}\text { Management } \\
\text { Recommendation } \\
\text { Year } t+1 \\
\end{array}$ \\
\hline Mixed & BENTON LAKE WMD & $\begin{array}{l}\text { TOOLE COUNTY } \\
\text { WPA }\end{array}$ & Furnell & 2009 & Rest & NA & Med & $2-4$ & $\{60-100, K B\}$ & NA \\
\hline Mixed & BENTON LAKE WMD & $\begin{array}{l}\text { TOOLE COUNTY } \\
\text { WPA }\end{array}$ & Furnell & 2010 & Graze & Graze & Med & 1 & $\{60-100, K B\}$ & Burn \\
\hline Mixed & BENTON LAKE WMD & $\begin{array}{l}\text { TOOLE COUNTY } \\
\text { WPA }\end{array}$ & Furnell & 2011 & Graze & Graze & Med & 1 & $\{60-100, K B\}$ & Graze \\
\hline Mixed & BENTON LAKE WMD & $\begin{array}{l}\text { TOOLE COUNTY } \\
\text { WPA }\end{array}$ & Furnell & 2012 & Rest & Rest & Med & $2-4$ & $\{45-60, K B\}$ & Graze \\
\hline Mixed & DEVILS LAKE WMD & $\begin{array}{c}\text { BENSON COUNTY } \\
\text { WPA }\end{array}$ & Melass South & 2009 & Rest & NA & Med & $2-4$ & $\{45-60, \mathrm{CO}\}$ & NA \\
\hline Mixed & DEVILS LAKE WMD & $\begin{array}{c}\text { BENSON COUNTY } \\
\text { WPA }\end{array}$ & Melass South & 2010 & Graze & Graze & High & 1 & $\{45-60, \mathrm{CO}\}$ & Burn \\
\hline Mixed & DEVILS LAKE WMD & $\begin{array}{c}\text { BENSON COUNTY } \\
\text { WPA }\end{array}$ & Melass South & 2011 & Graze & Rest & Med & $2-4$ & $\{30-45, S B\}$ & Graze \\
\hline Mixed & DEVILS LAKE WMD & $\begin{array}{c}\text { BENSON COUNTY } \\
\text { WPA }\end{array}$ & Melass South & 2012 & Burn & Burn & Med & 1 & $\{30-45, \mathrm{CO}\}$ & Graze \\
\hline Mixed & DEVILS LAKE WMD & $\begin{array}{c}\text { SULLYS HILL } \\
\text { NATIONAL GAME } \\
\text { DDECEDI/F }\end{array}$ & $\begin{array}{c}\text { Sullys Hill Native } \\
\text { Prairie }\end{array}$ & 2009 & Burn & NA & High & 1 & $\{60-100, K B\}$ & NA \\
\hline Mixed & DEVILS LAKE WMD & NATIONAL GAME & $\begin{array}{c}\text { Sullys Hill Native } \\
\text { Prairie }\end{array}$ & 2010 & Rest & Rest & Med & $2-4$ & $\{60-100, K B\}$ & Burn \\
\hline Mixed & DEVILS LAKE WMD & NATIONAL GAME & $\begin{array}{c}\text { Sullys Hill Native } \\
\text { Prairie }\end{array}$ & 2011 & Rest & Rest & Med & $2-4$ & $\{60-100, K B\}$ & Burn/Graze \\
\hline Mixed & DEVILS LAKE WMD & $\begin{array}{l}\text { NATIONAL GAME } \\
\text { DDECLDI/C }\end{array}$ & $\begin{array}{c}\text { Sullys Hill Native } \\
\text { Prairie }\end{array}$ & 2012 & Rest & Rest & Low & $2-4$ & $\{0-30, K B\}$ & Graze \\
\hline Mixed & DEVILS LAKE WMD & $\begin{array}{c}\text { TOWNER COUNTY } \\
\text { WPA }\end{array}$ & Towner & 2011 & Rest & Rest & Low & $2-4$ & $\{30-45, K B\}$ & Burn \\
\hline Mixed & DEVILS LAKE WMD & $\begin{array}{c}\text { TOWNER COUNTY } \\
\text { WPA }\end{array}$ & Towner & 2012 & Rest & Rest & Low & $2-4$ & $\{0-30, K B\}$ & Graze \\
\hline Mixed & HURON WMD & $\begin{array}{c}\text { BUFFALO COUNTY } \\
\text { WPA }\end{array}$ & Mills Unit 2 & 2009 & Graze & NA & Med & 1 & $\{45-60, S B\}$ & NA \\
\hline Mixed & HURON WMD & $\begin{array}{c}\text { BUFFALO COUNTY } \\
\text { WPA }\end{array}$ & Mills Unit 2 & 2010 & Graze & Graze & Med & 1 & $\{0-30, \mathrm{CO}\}$ & Graze \\
\hline
\end{tabular}




\begin{tabular}{|c|c|c|c|c|c|c|c|c|c|c|}
\hline $\begin{array}{l}\text { Grass } \\
\text { Type }\end{array}$ & Complex & Org & Unit & $\begin{array}{c}\text { Management } \\
\text { Year }\end{array}$ & $\begin{array}{c}\text { Management } \\
\text { Applied }\end{array}$ & $\begin{array}{c}\text { Management } \\
\text { Classified }\end{array}$ & $\begin{array}{c}\text { Defoliation } \\
\text { Level }\end{array}$ & $\begin{array}{l}\text { Years Since } \\
\text { Defoliation }\end{array}$ & $\begin{array}{l}\text { Vegetation } \\
\text { State }\end{array}$ & $\begin{array}{c}\text { Management } \\
\text { Recommendation } \\
\text { Year } t+1\end{array}$ \\
\hline Mixed & HURON WMD & $\begin{array}{c}\text { BUFFALO COUNTY } \\
\text { WPA }\end{array}$ & Mills Unit 2 & 2011 & Graze & Graze & Med & 1 & $\{30-45, \mathrm{CO}\}$ & Graze \\
\hline Mixed & HURON WMD & $\begin{array}{c}\text { BUFFALO COUNTY } \\
\text { WPA }\end{array}$ & Mills Unit 2 & 2012 & Graze & Graze & High & 1 & $\{0-30, \mathrm{CO}\}$ & Graze \\
\hline Mixed & HURON WMD & $\begin{array}{l}\text { HAND COUNTY } \\
\text { WPA }\end{array}$ & Campbell Unit 2 & 2009 & Graze & NA & High & 1 & $\{0-30, \mathrm{CO}\}$ & NA \\
\hline Mixed & HURON WMD & $\begin{array}{l}\text { HAND COUNTY } \\
\text { WPA }\end{array}$ & Campbell Unit 2 & 2010 & Rest & Rest & Med & $2-4$ & $\{0-30, K B\}$ & Burn \\
\hline Mixed & HURON WMD & $\begin{array}{c}\text { HAND COUNTY } \\
\text { WPA }\end{array}$ & Campbell Unit 2 & 2011 & Rest & Rest & Med & $2-4$ & $\{0-30, K B\}$ & Burn \\
\hline Mixed & HURON WMD & $\begin{array}{l}\text { HAND COUNTY } \\
\text { WPA }\end{array}$ & Campbell Unit 2 & 2012 & Rest & Rest & Med & $2-4$ & $\{0-30, \mathrm{CO}\}$ & Rest \\
\hline Mixed & HURON WMD & $\begin{array}{c}\text { HAND COUNTY } \\
\text { WPA }\end{array}$ & Millerdale Unit 2 & 2009 & Rest & NA & Med & $2-4$ & $\{0-30, K B\}$ & NA \\
\hline Mixed & HURON WMD & $\begin{array}{l}\text { HAND COUNTY } \\
\text { WPA }\end{array}$ & Millerdale Unit 2 & 2010 & Burn/Graze & Burn/Graze & Med & 1 & $\{0-30, K B\}$ & Burn \\
\hline Mixed & HURON WMD & $\begin{array}{c}\text { HAND COUNTY } \\
\text { WPA }\end{array}$ & Millerdale Unit 2 & 2011 & Graze & Graze & Med & 1 & $\{0-30, K B\}$ & Rest \\
\hline Mixed & HURON WMD & $\begin{array}{l}\text { HAND COUNTY } \\
\text { WPA }\end{array}$ & Millerdale Unit 2 & 2012 & Rest & Rest & Med & $2-4$ & $\{0-30, K B\}$ & Burn \\
\hline Mixed & HURON WMD & $\begin{array}{l}\text { HAND COUNTY } \\
\text { WPA }\end{array}$ & Slunecka Unit 3 & 2009 & Burn/Graze & NA & Med & 1 & $\{60-100, \mathrm{CO}\}$ & NA \\
\hline Mixed & HURON WMD & $\begin{array}{l}\text { HAND COUNTY } \\
\text { WPA }\end{array}$ & Slunecka Unit 3 & 2010 & Graze & Graze & Med & 1 & $\{60-100, K B\}$ & Burn \\
\hline Mixed & HURON WMD & $\begin{array}{l}\text { HAND COUNTY } \\
\text { WPA }\end{array}$ & Slunecka Unit 3 & 2011 & Graze & Graze & High & 1 & $\{30-45, K B\}$ & Burn/Graze \\
\hline Mixed & HURON WMD & $\begin{array}{l}\text { HAND COUNTY } \\
\text { WPA }\end{array}$ & Slunecka Unit 3 & 2012 & Graze & Graze & High & 1 & $\{45-60, K B\}$ & Rest \\
\hline Mixed & HURON WMD & $\begin{array}{c}\text { HAND COUNTY } \\
\text { WPA }\end{array}$ & Slunecka Unit 4 & 2009 & Burn/Graze & NA & Med & 1 & $\{30-45, \mathrm{CO}\}$ & NA \\
\hline Mixed & HURON WMD & $\begin{array}{l}\text { HAND COUNTY } \\
\text { WPA }\end{array}$ & Slunecka Unit 4 & 2010 & Graze & Graze & Med & 1 & $\{45-60, \mathrm{CO}\}$ & Graze \\
\hline
\end{tabular}




\begin{tabular}{|c|c|c|c|c|c|c|c|c|c|c|}
\hline $\begin{array}{l}\text { Grass } \\
\text { Type }\end{array}$ & Complex & Org & Unit & $\begin{array}{c}\text { Management } \\
\text { Year }\end{array}$ & $\begin{array}{c}\text { Management } \\
\text { Applied }\end{array}$ & $\begin{array}{c}\text { Management } \\
\text { Classified }\end{array}$ & $\begin{array}{c}\text { Defoliation } \\
\text { Level }\end{array}$ & $\begin{array}{l}\text { Years Since } \\
\text { Defoliation }\end{array}$ & $\begin{array}{l}\text { Vegetation } \\
\text { State }\end{array}$ & $\begin{array}{c}\text { Management } \\
\text { Recommendation } \\
\text { Year } t+1\end{array}$ \\
\hline Mixed & HURON WMD & $\begin{array}{c}\text { HAND COUNTY } \\
\text { WPA }\end{array}$ & Slunecka Unit 4 & 2011 & Graze & Graze & High & 1 & $\{0-30, K B\}$ & Graze \\
\hline Mixed & HURON WMD & $\begin{array}{c}\text { HAND COUNTY } \\
\text { WPA }\end{array}$ & Slunecka Unit 4 & 2012 & Graze & Graze & High & 1 & $\{30-45, \mathrm{~KB}\}$ & Burn/Graze \\
\hline Mixed & HURON WMD & $\begin{array}{c}\text { HAND COUNTY } \\
\text { WPA }\end{array}$ & VenJohn Unit 1 & 2009 & Graze & NA & Med & 1 & $\{0-30, K B\}$ & NA \\
\hline Mixed & HURON WMD & $\begin{array}{c}\text { HAND COUNTY } \\
\text { WPA }\end{array}$ & VenJohn Unit 1 & 2010 & Rest & Rest & Med & $2-4$ & $\{0-30, K B\}$ & Burn \\
\hline Mixed & HURON WMD & $\begin{array}{c}\text { HAND COUNTY } \\
\text { WPA }\end{array}$ & VenJohn Unit 1 & 2011 & Graze & Graze & High & 1 & $\{0-30, K B\}$ & Graze \\
\hline Mixed & HURON WMD & $\begin{array}{c}\text { HAND COUNTY } \\
\text { WPA }\end{array}$ & VenJohn Unit 1 & 2012 & Burn/Graze & Burn/Graze & High & 1 & $\{0-30, K B\}$ & Graze \\
\hline Mixed & HURON WMD & HYDE COUNTY WPA & Cowan Unit 4 & 2009 & Graze & NA & High & 1 & $\{30-45, \mathrm{~KB}\}$ & NA \\
\hline Mixed & HURON WMD & HYDE COUNTY WPA & Cowan Unit 4 & 2010 & Graze & Graze & High & 1 & $\{0-30, K B\}$ & Burn \\
\hline Mixed & HURON WMD & HYDE COUNTY WPA & Cowan Unit 4 & 2011 & Burn/Graze & Burn/Graze & High & 1 & $\{30-45, \mathrm{~KB}\}$ & Burn/Graze \\
\hline Mixed & HURON WMD & HYDE COUNTY WPA & Cowan Unit 6 & 2009 & Burn/Graze & NA & High & 1 & NA & NA \\
\hline Mixed & HURON WMD & HYDE COUNTY WPA & Cowan Unit 6 & 2010 & Graze & Graze & High & 1 & $\{0-30, \mathrm{CO}\}$ & Graze \\
\hline Mixed & HURON WMD & HYDE COUNTY WPA & Cowan Unit 6 & 2011 & Graze & Graze & High & 1 & $\{0-30, \mathrm{CO}\}$ & Graze \\
\hline Mixed & HURON WMD & HYDE COUNTY WPA & Harter Unit 6 & 2009 & Burn & NA & High & 1 & $\{30-45, \mathrm{~KB}\}$ & NA \\
\hline Mixed & HURON WMD & HYDE COUNTY WPA & Harter Unit 6 & 2010 & Rest & Rest & High & $2-4$ & $\{0-30, K B\}$ & Burn \\
\hline Mixed & HURON WMD & HYDE COUNTY WPA & Harter Unit 6 & 2011 & Graze & Graze & High & 1 & $\{0-30, K B\}$ & Graze \\
\hline Mixed & HURON WMD & HYDE COUNTY WPA & Harter Unit 6 & 2012 & Graze & Graze & High & 1 & $\{0-30, K B\}$ & Graze \\
\hline
\end{tabular}




\begin{tabular}{|c|c|c|c|c|c|c|c|c|c|c|}
\hline $\begin{array}{l}\text { Grass } \\
\text { Type }\end{array}$ & Complex & Org & Unit & $\begin{array}{c}\text { Management } \\
\text { Year }\end{array}$ & $\begin{array}{c}\text { Management } \\
\text { Applied }\end{array}$ & $\begin{array}{c}\text { Management } \\
\text { Classified }\end{array}$ & $\begin{array}{c}\text { Defoliation } \\
\text { Level }\end{array}$ & $\begin{array}{l}\text { Years Since } \\
\text { Defoliation }\end{array}$ & $\begin{array}{l}\text { Vegetation } \\
\text { State }\end{array}$ & $\begin{array}{c}\text { Management } \\
\text { Recommendation } \\
\text { Year } t+1\end{array}$ \\
\hline Mixed & HURON WMD & $\begin{array}{c}\text { JERAULD COUNTY } \\
\text { WPA }\end{array}$ & Winter & 2009 & Burn/Graze & NA & High & 1 & $\{0-30, \mathrm{CO}\}$ & NA \\
\hline Mixed & HURON WMD & $\begin{array}{c}\text { JERAULD COUNTY } \\
\text { WPA }\end{array}$ & Winter & 2010 & Rest & Rest & Med & $2-4$ & $\{0-30, K B\}$ & Burn \\
\hline Mixed & HURON WMD & $\begin{array}{c}\text { JERAULD COUNTY } \\
\text { WPA }\end{array}$ & Winter & 2011 & Graze & Graze & High & 1 & $\{0-30, K B\}$ & Graze \\
\hline Mixed & HURON WMD & $\begin{array}{c}\text { JERAULD COUNTY } \\
\text { WPA }\end{array}$ & Winter & 2012 & Graze & Graze & High & 1 & $\{0-30, K B\}$ & Graze \\
\hline Mixed & KULM WMD & $\begin{array}{c}\text { DICKEY COUNTY } \\
\text { WPA }\end{array}$ & Lazy M Unit 3 & 2012 & Burn/Graze & Burn & High & 1 & $\{0-30, S B\}$ & Graze \\
\hline Mixed & KULM WMD & $\begin{array}{c}\text { LA MOURE COUNTY } \\
\text { WPA }\end{array}$ & Cornell 2 & 2009 & Graze & NA & Med & 1 & $\{0-30, K B\}$ & NA \\
\hline Mixed & KULM WMD & $\begin{array}{c}\text { LA MOURE COUNTY } \\
\text { WPA }\end{array}$ & Cornell 2 & 2010 & Rest & Rest & Med & $2-4$ & $\{0-30, \mathrm{RM}\}$ & Burn \\
\hline Mixed & KULM WMD & $\begin{array}{c}\text { LA MOURE COUNTY } \\
\text { WPA }\end{array}$ & Cornell 2 & 2011 & Burn/Graze & Burn/Graze & Med & 1 & $\{0-30, K B\}$ & Rest \\
\hline Mixed & KULM WMD & $\begin{array}{c}\text { LA MOURE COUNTY } \\
\text { WPA }\end{array}$ & Cornell 2 & 2012 & Graze & Graze & High & 1 & $\{0-30, K B\}$ & Graze \\
\hline Mixed & KULM WMD & $\begin{array}{c}\text { LA MOURE COUNTY } \\
\text { WPA }\end{array}$ & Cornell 3 & 2009 & Rest & NA & Low & $2-4$ & $\{30-45, \mathrm{CO}\}$ & NA \\
\hline Mixed & KULM WMD & $\begin{array}{c}\text { LA MOURE COUNTY } \\
\text { WPA }\end{array}$ & Cornell 3 & 2010 & Rest & Rest & Low & $2-4$ & $\{60-100, K B\}$ & Burn \\
\hline Mixed & KULM WMD & $\begin{array}{c}\text { LA MOURE COUNTY } \\
\text { WPA }\end{array}$ & Cornell 3 & 2011 & Burn/Graze & Burn/Graze & Med & 1 & $\{30-45, \mathrm{CO}\}$ & Graze \\
\hline Mixed & KULM WMD & $\begin{array}{c}\text { LA MOURE COUNTY } \\
\text { WPA }\end{array}$ & Cornell 3 & 2012 & Graze & Graze & Med & 1 & $\{0-30, K B\}$ & Rest \\
\hline Mixed & KULM WMD & $\begin{array}{l}\text { LOGAN COUNTY } \\
\text { WPA }\end{array}$ & Kroll 1 & 2009 & Graze & NA & Med & 1 & $\{0-30, \mathrm{CO}\}$ & NA \\
\hline Mixed & KULM WMD & $\begin{array}{l}\text { LOGAN COUNTY } \\
\text { WPA }\end{array}$ & Kroll 1 & 2010 & Burn & Burn & Med & 1 & $\{0-30, S B\}$ & Graze \\
\hline Mixed & KULM WMD & $\begin{array}{l}\text { LOGAN COUNTY } \\
\text { WPA }\end{array}$ & Kroll 1 & 2011 & Rest & Rest & Med & $2-4$ & $\{0-30, S B\}$ & Graze \\
\hline
\end{tabular}




\begin{tabular}{|c|c|c|c|c|c|c|c|c|c|c|}
\hline $\begin{array}{l}\text { Grass } \\
\text { Type }\end{array}$ & Complex & Org & Unit & $\begin{array}{c}\text { Management } \\
\text { Year }\end{array}$ & $\begin{array}{c}\text { Management } \\
\text { Applied }\end{array}$ & $\begin{array}{c}\text { Management } \\
\text { Classified }\end{array}$ & $\begin{array}{c}\text { Defoliation } \\
\text { Level }\end{array}$ & $\begin{array}{l}\text { Years Since } \\
\text { Defoliation }\end{array}$ & $\begin{array}{l}\text { Vegetation } \\
\text { State }\end{array}$ & $\begin{array}{c}\text { Management } \\
\text { Recommendation } \\
\text { Year } t+1\end{array}$ \\
\hline Mixed & KULM WMD & $\begin{array}{c}\text { LOGAN COUNTY } \\
\text { WPA }\end{array}$ & Mayer 2 & 2009 & Graze & NA & Med & 1 & $\{0-30, K B\}$ & NA \\
\hline Mixed & KULM WMD & $\begin{array}{l}\text { LOGAN COUNTY } \\
\text { WPA }\end{array}$ & Mayer 2 & 2010 & Rest & Rest & Low & $2-4$ & $\{0-30, K B\}$ & Burn \\
\hline Mixed & KULM WMD & $\begin{array}{l}\text { LOGAN COUNTY } \\
\text { WPA }\end{array}$ & Mayer 2 & 2011 & Burn & Burn & Med & 1 & $\{45-60, \mathrm{~KB}\}$ & Burn \\
\hline Mixed & KULM WMD & $\begin{array}{l}\text { LOGAN COUNTY } \\
\text { WPA }\end{array}$ & Mayer 2 & 2012 & Burn & Burn & Med & 1 & $\{0-30, K B\}$ & Rest \\
\hline Mixed & KULM WMD & $\begin{array}{l}\text { LOGAN COUNTY } \\
\text { WPA }\end{array}$ & Mayer 3 & 2009 & Rest & NA & Low & $2-4$ & $\{0-30, \mathrm{CO}\}$ & NA \\
\hline Mixed & KULM WMD & $\begin{array}{l}\text { LOGAN COUNTY } \\
\text { WPA }\end{array}$ & Mayer 3 & 2010 & Graze & Graze & Med & 1 & $\{0-30, K B\}$ & Burn \\
\hline Mixed & KULM WMD & $\begin{array}{l}\text { LOGAN COUNTY } \\
\text { WPA }\end{array}$ & Mayer 3 & 2011 & Burn & Burn & Med & 1 & $\{45-60, \mathrm{CO}\}$ & Rest \\
\hline Mixed & KULM WMD & $\begin{array}{l}\text { LOGAN COUNTY } \\
\text { WPA }\end{array}$ & Mayer 3 & 2012 & Burn & Burn & Med & 1 & $\{0-30, \mathrm{CO}\}$ & Rest \\
\hline Mixed & KULM WMD & $\begin{array}{l}\text { LOGAN COUNTY } \\
\text { WPA }\end{array}$ & Mayer 4 & 2009 & Rest & NA & Med & $2-4$ & $\{0-30, K B\}$ & NA \\
\hline Mixed & KULM WMD & $\begin{array}{l}\text { LOGAN COUNTY } \\
\text { WPA }\end{array}$ & Mayer 4 & 2010 & Rest & Rest & Med & $2-4$ & $\{0-30, K B\}$ & Burn \\
\hline Mixed & KULM WMD & $\begin{array}{l}\text { LOGAN COUNTY } \\
\text { WPA }\end{array}$ & Mayer 4 & 2011 & Burn & Burn & Med & 1 & $\{30-45, \mathrm{CO}\}$ & Graze \\
\hline Mixed & KULM WMD & $\begin{array}{l}\text { LOGAN COUNTY } \\
\text { WPA }\end{array}$ & Mayer 4 & 2012 & Burn/Graze & Burn/Graze & Med & 1 & $\{0-30, \mathrm{CO}\}$ & Rest \\
\hline Mixed & KULM WMD & $\begin{array}{c}\text { MCINTOSH COUNTY } \\
\text { WPA }\end{array}$ & Geiszler 1 & 2009 & Graze & NA & High & 1 & $\{0-30, \mathrm{CO}\}$ & NA \\
\hline Mixed & KULM WMD & $\begin{array}{c}\text { MCINTOSH COUNTY } \\
\text { WPA }\end{array}$ & Geiszler 1 & 2010 & Rest & Rest & High & $2-4$ & $\{30-45, \mathrm{CO}\}$ & Graze \\
\hline Mixed & KULM WMD & $\begin{array}{c}\text { MCINTOSH COUNTY } \\
\text { WPA }\end{array}$ & Geiszler 1 & 2011 & Burn/Graze & Burn/Graze & High & 1 & $\{0-30, \mathrm{CO}\}$ & Graze \\
\hline Mixed & KULM WMD & $\begin{array}{c}\text { MCINTOSH COUNTY } \\
\text { WPA }\end{array}$ & Geiszler 1 & 2012 & Rest & Rest & High & $2-4$ & $\{0-30, \mathrm{CO}\}$ & Graze \\
\hline
\end{tabular}




\begin{tabular}{|c|c|c|c|c|c|c|c|c|c|c|}
\hline $\begin{array}{l}\text { Grass } \\
\text { Type }\end{array}$ & Complex & Org & Unit & $\begin{array}{c}\text { Management } \\
\text { Year }\end{array}$ & $\begin{array}{c}\text { Management } \\
\text { Applied }\end{array}$ & $\begin{array}{c}\text { Management } \\
\text { Classified }\end{array}$ & $\begin{array}{c}\text { Defoliation } \\
\text { Level }\end{array}$ & $\begin{array}{l}\text { Years Since } \\
\text { Defoliation }\end{array}$ & $\begin{array}{l}\text { Vegetation } \\
\text { State }\end{array}$ & $\begin{array}{c}\text { Management } \\
\text { Recommendation } \\
\text { Year } t+1\end{array}$ \\
\hline Mixed & KULM WMD & $\begin{array}{c}\text { MCINTOSH COUNTY } \\
\text { WPA }\end{array}$ & Geiszler 2 & 2009 & Burn & NA & High & 1 & $\{0-30, K B\}$ & NA \\
\hline Mixed & KULM WMD & $\begin{array}{c}\text { MCINTOSH COUNTY } \\
\text { WPA }\end{array}$ & Geiszler 2 & 2010 & Graze & Graze & High & 1 & $\{0-30, K B\}$ & Burn \\
\hline Mixed & KULM WMD & $\begin{array}{c}\text { MCINTOSH COUNTY } \\
\text { WPA }\end{array}$ & Geiszler 2 & 2011 & Graze & Graze & High & 1 & $\{0-30, K B\}$ & Graze \\
\hline Mixed & KULM WMD & $\begin{array}{c}\text { MCINTOSH COUNTY } \\
\text { WPA }\end{array}$ & Geiszler 2 & 2012 & Rest & Rest & High & $2-4$ & $\{0-30, K B\}$ & Graze \\
\hline Mixed & KULM WMD & $\begin{array}{c}\text { MCINTOSH COUNTY } \\
\text { WPA }\end{array}$ & Geiszler 3 & 2009 & hay & NA & High & 1 & $\{0-30, K B\}$ & NA \\
\hline Mixed & KULM WMD & $\begin{array}{c}\text { MCINTOSH COUNTY } \\
\text { WPA }\end{array}$ & Geiszler 3 & 2010 & Graze & Graze & High & 1 & $\{0-30, K B\}$ & Burn \\
\hline Mixed & KULM WMD & $\begin{array}{c}\text { MCINTOSH COUNTY } \\
\text { WPA }\end{array}$ & Geiszler 3 & 2011 & Burn & Unclassifiable & High & 1 & $\{45-60, K B\}$ & Rest \\
\hline Mixed & KULM WMD & $\begin{array}{c}\text { MCINTOSH COUNTY } \\
\text { WPA }\end{array}$ & Geiszler 3 & 2012 & Graze & Graze & High & 1 & $\{0-30, K B\}$ & Graze \\
\hline Mixed & KULM WMD & $\begin{array}{c}\text { MCINTOSH COUNTY } \\
\text { WPA }\end{array}$ & Geiszler 4 & 2009 & Graze & NA & High & 1 & $\{0-30, K B\}$ & NA \\
\hline Mixed & KULM WMD & $\begin{array}{c}\text { MCINTOSH COUNTY } \\
\text { WPA }\end{array}$ & Geiszler 4 & 2010 & Rest & Rest & High & $2-4$ & $\{0-30, K B\}$ & Burn \\
\hline Mixed & KULM WMD & $\begin{array}{c}\text { MCINTOSH COUNTY } \\
\text { WPA }\end{array}$ & Geiszler 4 & 2011 & Rest & Rest & Med & $2-4$ & $\{0-30, K B\}$ & Burn \\
\hline Mixed & KULM WMD & $\begin{array}{c}\text { MCINTOSH COUNTY } \\
\text { WPA }\end{array}$ & Geiszler 4 & 2012 & Burn/Graze & Burn/Graze & High & 1 & $\{0-30, \mathrm{CO}\}$ & Graze \\
\hline Mixed & LAKE ANDES NWR & $\begin{array}{c}\text { AURORA COUNTY } \\
\text { WPA }\end{array}$ & Foster & 2009 & Rest & NA & Low & $2-4$ & $\{0-30, S B\}$ & NA \\
\hline Mixed & LAKE ANDES NWR & $\begin{array}{l}\text { AURORA COUNTY } \\
\text { WPA }\end{array}$ & Foster & 2010 & Rest & Rest & Low & $2-4$ & $\{0-30, S B\}$ & Graze \\
\hline Mixed & LAKE ANDES NWR & $\begin{array}{c}\text { AURORA COUNTY } \\
\text { WPA }\end{array}$ & Foster & 2011 & Graze & Unclassifiable & Low & 1 & $\{0-30, S B\}$ & Graze \\
\hline Mixed & LAKE ANDES NWR & $\begin{array}{c}\text { AURORA COUNTY } \\
\text { WPA }\end{array}$ & Foster & 2012 & Graze & Unclassifiable & Med & 1 & $\{0-30, S B\}$ & Graze \\
\hline
\end{tabular}




\begin{tabular}{|c|c|c|c|c|c|c|c|c|c|c|}
\hline $\begin{array}{l}\text { Grass } \\
\text { Type }\end{array}$ & Complex & Org & Unit & $\begin{array}{c}\text { Management } \\
\text { Year }\end{array}$ & $\begin{array}{c}\text { Management } \\
\text { Applied }\end{array}$ & $\begin{array}{l}\text { Management } \\
\text { Classified }\end{array}$ & $\begin{array}{l}\text { Defoliation } \\
\text { Level }\end{array}$ & $\begin{array}{l}\text { Years Since } \\
\text { Defoliation }\end{array}$ & $\begin{array}{c}\text { Vegetation } \\
\text { State }\end{array}$ & $\begin{array}{c}\text { Management } \\
\text { Recommendation } \\
\text { Year } t+1 \\
\end{array}$ \\
\hline Mixed & LAKE ANDES NWR & $\begin{array}{l}\text { BON HOMME } \\
\text { COUNTY WPA }\end{array}$ & Hieb & 2009 & Burn & NA & Low & 1 & $\{45-60, S B\}$ & NA \\
\hline Mixed & LAKE ANDES NWR & $\begin{array}{l}\text { BON HOMME } \\
\text { COUNTY WPA }\end{array}$ & Hieb & 2010 & reset & Rest & Low & $2-4$ & $\{30-45, \mathrm{SB}\}$ & Graze \\
\hline Mixed & LAKE ANDES NWR & $\begin{array}{l}\text { BON HOMME } \\
\text { COUNTY WPA }\end{array}$ & Hieb & 2011 & Graze & Graze & Low & 1 & $\{60-100, S B\}$ & Graze \\
\hline Mixed & LAKE ANDES NWR & $\begin{array}{l}\text { BON HOMME } \\
\text { COUNTY WPA }\end{array}$ & Hieb & 2012 & Graze & Graze & Med & 1 & $\{60-100, S B\}$ & Graze \\
\hline Mixed & LAKE ANDES NWR & $\begin{array}{l}\text { CHARLES MIX } \\
\text { COUNTY WPA }\end{array}$ & Trout & 2011 & Rest & Rest & Med & $2-4$ & $\{0-30, S B\}$ & Graze \\
\hline Mixed & LAKE ANDES NWR & $\begin{array}{l}\text { CHARLES MIX } \\
\text { COUNTY WPA }\end{array}$ & Trout & 2012 & Graze & Graze & Med & 1 & $\{0-30, S B\}$ & Graze \\
\hline Mixed & LAKE ANDES NWR & $\begin{array}{c}\text { DOUGLAS COUNTY } \\
\text { WPA }\end{array}$ & Denning & 2010 & Rest & Rest & Low & $2-4$ & $\{0-30, S B\}$ & Graze \\
\hline Mixed & LAKE ANDES NWR & $\begin{array}{c}\text { DOUGLAS COUNTY } \\
\text { WPA }\end{array}$ & Denning & 2011 & Graze & Unclassifiable & Low & 1 & $\{0-30, S B\}$ & Graze \\
\hline Mixed & LAKE ANDES NWR & $\begin{array}{c}\text { DOUGLAS COUNTY } \\
\text { WPA }\end{array}$ & Denning & 2012 & Graze & Graze & Med & 1 & $\{0-30, S B\}$ & Graze \\
\hline Mixed & LONG LAKE WMD & $\begin{array}{c}\text { BURLEIGH COUNTY } \\
\text { WPA }\end{array}$ & $\begin{array}{l}\text { Crimmins NE } \\
\text { Grazing Unit }\end{array}$ & 2009 & Graze & NA & Med & 1 & $\{0-30, \mathrm{CO}\}$ & NA \\
\hline Mixed & LONG LAKE WMD & $\begin{array}{c}\text { BURLEIGH COUNTY } \\
\text { WPA }\end{array}$ & $\begin{array}{l}\text { Crimmins NE } \\
\text { Grazing Unit }\end{array}$ & 2010 & Rest & Rest & Med & $2-4$ & $\{0-30, \mathrm{CO}\}$ & Graze \\
\hline Mixed & LONG LAKE WMD & $\begin{array}{c}\text { BURLEIGH COUNTY } \\
\text { WPA }\end{array}$ & $\begin{array}{l}\text { Crimmins NE } \\
\text { Grazing Unit }\end{array}$ & 2011 & Burn & Burn & Med & 1 & $\{0-30, \mathrm{CO}\}$ & Rest \\
\hline Mixed & LONG LAKE WMD & $\begin{array}{c}\text { BURLEIGH COUNTY } \\
\text { WPA }\end{array}$ & $\begin{array}{l}\text { Crimmins NE } \\
\text { Grazing Unit }\end{array}$ & 2012 & Rest & Rest & Med & $2-4$ & $\{0-30, \mathrm{CO}\}$ & Rest \\
\hline Mixed & LONG LAKE WMD & $\begin{array}{c}\text { BURLEIGH COUNTY } \\
\text { WPA }\end{array}$ & $\begin{array}{c}\text { Rath WPA - \#1 } \\
\text { Grazing Unit }\end{array}$ & 2009 & Burn & NA & Low & 1 & $\{45-60, S B\}$ & NA \\
\hline Mixed & LONG LAKE WMD & $\begin{array}{c}\text { BURLEIGH COUNTY } \\
\text { WPA }\end{array}$ & $\begin{array}{c}\text { Rath WPA - \#1 } \\
\text { Grazing Unit }\end{array}$ & 2010 & Graze & Graze & Med & 1 & $\{30-45, \mathrm{CO}\}$ & Graze \\
\hline Mixed & LONG LAKE WMD & $\begin{array}{c}\text { BURLEIGH COUNTY } \\
\text { WPA }\end{array}$ & $\begin{array}{l}\text { Rath WPA - \#1 } \\
\text { Grazing Unit }\end{array}$ & 2011 & Graze & Graze & Med & 1 & $\{30-45, \mathrm{CO}\}$ & Graze \\
\hline
\end{tabular}




\begin{tabular}{|c|c|c|c|c|c|c|c|c|c|c|}
\hline $\begin{array}{l}\text { Grass } \\
\text { Type }\end{array}$ & Complex & Org & Unit & $\begin{array}{c}\text { Management } \\
\text { Year }\end{array}$ & $\begin{array}{c}\text { Management } \\
\text { Applied }\end{array}$ & $\begin{array}{l}\text { Management } \\
\text { Classified }\end{array}$ & $\begin{array}{l}\text { Defoliation } \\
\text { Level }\end{array}$ & $\begin{array}{l}\text { Years Since } \\
\text { Defoliation }\end{array}$ & $\begin{array}{l}\text { Vegetation } \\
\text { State }\end{array}$ & $\begin{array}{c}\text { Management } \\
\text { Recommendation } \\
\text { Year } t+1\end{array}$ \\
\hline Mixed & LONG LAKE WMD & $\begin{array}{c}\text { BURLEIGH COUNTY } \\
\text { WPA }\end{array}$ & $\begin{array}{c}\text { Rath WPA - \#1 } \\
\text { Grazing Unit }\end{array}$ & 2012 & Graze & Graze & Med & 1 & $\{30-45, \mathrm{CO}\}$ & Graze \\
\hline Mixed & LONG LAKE WMD & $\begin{array}{l}\text { KIDDER COUNTY } \\
\text { WPA }\end{array}$ & $\begin{array}{c}\text { Thacker WPA- } \\
\text { North }\end{array}$ & 2009 & Rest & NA & Low & $2-4$ & $\{0-30, \mathrm{CO}\}$ & NA \\
\hline Mixed & LONG LAKE WMD & $\begin{array}{l}\text { KIDDER COUNTY } \\
\text { WPA }\end{array}$ & $\begin{array}{c}\text { Thacker WPA- } \\
\text { North }\end{array}$ & 2010 & Graze & Graze & Med & 1 & $\{0-30, \mathrm{CO}\}$ & Graze \\
\hline Mixed & LONG LAKE WMD & $\begin{array}{l}\text { KIDDER COUNTY } \\
\text { WPA }\end{array}$ & $\begin{array}{c}\text { Thacker WPA- } \\
\text { North }\end{array}$ & 2011 & Graze & Graze & Med & 1 & $\{0-30, \mathrm{CO}\}$ & Rest \\
\hline Mixed & LONG LAKE WMD & $\begin{array}{l}\text { KIDDER COUNTY } \\
\text { WPA }\end{array}$ & $\begin{array}{c}\text { Thacker WPA- } \\
\text { North }\end{array}$ & 2012 & Rest & Rest & Med & $2-4$ & $\{0-30, S B\}$ & Graze \\
\hline Mixed & LONG LAKE WMD & $\begin{array}{l}\text { KIDDER COUNTY } \\
\text { WPA }\end{array}$ & $\begin{array}{l}\text { Thacker WPA- } \\
\text { South }\end{array}$ & 2009 & Rest & NA & Low & $2-4$ & $\{0-30, S B\}$ & NA \\
\hline Mixed & LONG LAKE WMD & $\begin{array}{l}\text { KIDDER COUNTY } \\
\text { WPA }\end{array}$ & $\begin{array}{c}\text { Thacker WPA- } \\
\text { South }\end{array}$ & 2010 & Graze & Graze & Med & 1 & $\{0-30, \mathrm{CO}\}$ & Graze \\
\hline Mixed & LONG LAKE WMD & $\begin{array}{l}\text { KIDDER COUNTY } \\
\text { WPA }\end{array}$ & $\begin{array}{l}\text { Thacker WPA- } \\
\text { South }\end{array}$ & 2011 & Graze & Graze & Med & 1 & $\{0-30, S B\}$ & Graze \\
\hline Mixed & LONG LAKE WMD & $\begin{array}{l}\text { KIDDER COUNTY } \\
\text { WPA }\end{array}$ & $\begin{array}{c}\text { Thacker WPA- } \\
\text { South }\end{array}$ & 2012 & Graze & Graze & Med & 1 & $\{0-30, S B\}$ & Graze \\
\hline Mixed & LONG LAKE WMD & LONG LAKE NWR & G-12A East & 2009 & Graze & NA & Med & 1 & $\{0-30, \mathrm{CO}\}$ & NA \\
\hline Mixed & LONG LAKE WMD & LONG LAKE NWR & G-12A East & 2010 & Graze & Graze & High & 1 & $\{0-30, K B\}$ & Burn \\
\hline Mixed & LONG LAKE WMD & LONG LAKE NWR & G-12A East & 2011 & Graze & Graze & High & 1 & $\{0-30, \mathrm{CO}\}$ & Graze \\
\hline Mixed & LONG LAKE WMD & LONG LAKE NWR & G-12A East & 2012 & Graze & Graze & High & 1 & $\{0-30, \mathrm{CO}\}$ & Graze \\
\hline Mixed & LOSTWOOD COMPLEX & $\begin{array}{l}\text { BURKE COUNTY } \\
\text { WPA }\end{array}$ & Swanson & 2011 & Burn & Burn & Low & 1 & $\{45-60, S B\}$ & Graze \\
\hline Mixed & LOSTWOOD COMPLEX & $\begin{array}{l}\text { BURKE COUNTY } \\
\text { WPA }\end{array}$ & Swanson & 2012 & Graze & Graze & Med & 1 & $\{0-30, \mathrm{CO}\}$ & Rest \\
\hline Mixed & LOSTWOOD COMPLEX & LAKE ZAHL NWR & Lake Zahl 7 & 2009 & Burn & NA & Med & 1 & $\{45-60, S B\}$ & NA \\
\hline
\end{tabular}




\begin{tabular}{|c|c|c|c|c|c|c|c|c|c|c|}
\hline $\begin{array}{l}\text { Grass } \\
\text { Type }\end{array}$ & Complex & Org & Unit & $\begin{array}{c}\text { Management } \\
\text { Year }\end{array}$ & $\begin{array}{c}\text { Management } \\
\text { Applied }\end{array}$ & $\begin{array}{l}\text { Management } \\
\text { Classified }\end{array}$ & $\begin{array}{l}\text { Defoliation } \\
\text { Level }\end{array}$ & $\begin{array}{l}\text { Years Since } \\
\text { Defoliation }\end{array}$ & $\begin{array}{l}\text { Vegetation } \\
\text { State }\end{array}$ & $\begin{array}{c}\text { Management } \\
\text { Recommendation } \\
\text { Year } t+1 \\
\end{array}$ \\
\hline Mixed & LOSTWOOD COMPLEX & LAKE ZAHL NWR & Lake Zahl 7 & 2010 & Rest & Rest & Med & $2-4$ & $\{60-100, S B\}$ & Graze \\
\hline Mixed & LOSTWOOD COMPLEX & LAKE ZAHL NWR & Lake Zahl 7 & 2011 & Rest & Rest & Med & $2-4$ & $\{30-45, S B\}$ & Graze \\
\hline Mixed & LOSTWOOD COMPLEX & LAKE ZAHL NWR & Lake Zahl 7 & 2012 & Graze & Graze & Med & 1 & $\{0-30, \mathrm{CO}\}$ & Rest \\
\hline Mixed & LOSTWOOD COMPLEX & LOSTWOOD NWR & $\begin{array}{c}\text { Windmill South - } \\
\text { West half }\end{array}$ & 2009 & Burn & NA & Low & 1 & $\{30-45, \mathrm{CO}\}$ & NA \\
\hline Mixed & LOSTWOOD COMPLEX & LOSTWOOD NWR & $\begin{array}{c}\text { Windmill South - } \\
\text { West half }\end{array}$ & 2010 & Rest & Rest & Low & $2-4$ & $\{45-60, \mathrm{CO}\}$ & Burn \\
\hline Mixed & LOSTWOOD COMPLEX & LOSTWOOD NWR & $\begin{array}{c}\text { Windmill South - } \\
\text { West half }\end{array}$ & 2011 & Rest & Rest & Low & $2-4$ & $\{60-100, C O\}$ & Graze \\
\hline Mixed & LOSTWOOD COMPLEX & LOSTWOOD NWR & $\begin{array}{c}\text { Windmill South - } \\
\text { West half }\end{array}$ & 2012 & Rest & Rest & Low & $2-4$ & $\{0-30, \mathrm{CO}\}$ & Graze \\
\hline Mixed & LOSTWOOD COMPLEX & $\begin{array}{l}\text { MOUNTRAIL } \\
\text { COUNTY WPA }\end{array}$ & $\begin{array}{c}\text { Coteau Prairie - } \\
\text { G2 West half }\end{array}$ & 2009 & Rest & NA & Low & $2-4$ & $\{45-60, \mathrm{CO}\}$ & NA \\
\hline Mixed & LOSTWOOD COMPLEX & $\begin{array}{l}\text { MOUNTRAIL } \\
\text { COUNTY WPA }\end{array}$ & $\begin{array}{l}\text { Coteau Prairie - } \\
\text { G2 West half }\end{array}$ & 2010 & Burn & Burn & Med & 1 & $\{60-100, \mathrm{CO}\}$ & Burn \\
\hline Mixed & LOSTWOOD COMPLEX & $\begin{array}{l}\text { MOUNTRAIL } \\
\text { COUNTY WPA }\end{array}$ & $\begin{array}{l}\text { Coteau Prairie - } \\
\text { G2 West half }\end{array}$ & 2011 & Burn & Burn & Med & 1 & $\{30-45, \mathrm{CO}\}$ & Graze \\
\hline Mixed & LOSTWOOD COMPLEX & $\begin{array}{l}\text { MOUNTRAIL } \\
\text { COUNTY WPA }\end{array}$ & $\begin{array}{l}\text { Coteau Prairie - } \\
\text { G2 West half }\end{array}$ & 2012 & Graze & Graze & Med & 1 & $\{30-45, \mathrm{CO}\}$ & Graze \\
\hline Mixed & $\begin{array}{l}\text { MEDICINE LAKE NWR } \\
\text { COMPLEX }\end{array}$ & $\begin{array}{l}\text { MEDICINE LAKE } \\
\text { NWR }\end{array}$ & $\begin{array}{c}\text { East ML } \\
\text { Bridgerman }\end{array}$ & 2010 & Rest & Rest & Med & $2-4$ & $\{30-45, \mathrm{RM}\}$ & Graze \\
\hline Mixed & $\begin{array}{c}\text { MEDICINE LAKE NWR } \\
\text { COMPLEX }\end{array}$ & $\begin{array}{l}\text { MEDICINE LAKE } \\
\text { NWR }\end{array}$ & $\begin{array}{c}\text { East ML } \\
\text { Bridgerman }\end{array}$ & 2011 & Burn & Burn & High & 1 & $\{45-60, \mathrm{CO}\}$ & Graze \\
\hline Mixed & $\begin{array}{c}\text { MEDICINE LAKE NWR } \\
\text { COMPLEX }\end{array}$ & $\begin{array}{l}\text { MEDICINE LAKE } \\
\text { NWR }\end{array}$ & $\begin{array}{l}\text { East ML } \\
\text { Bridgerman }\end{array}$ & 2012 & Graze & Graze & High & 1 & $\{30-45, \mathrm{RM}\}$ & Graze \\
\hline Mixed & $\begin{array}{c}\text { MEDICINE LAKE NWR } \\
\text { COMPLEX }\end{array}$ & $\begin{array}{l}\text { MEDICINE LAKE } \\
\text { NWR }\end{array}$ & EAST ML LAKE 10 & 2010 & Graze & Graze & Med & 1 & $\{30-45, S B\}$ & Graze \\
\hline Mixed & $\begin{array}{l}\text { MEDICINE LAKE NWR } \\
\text { COMPLEX }\end{array}$ & $\begin{array}{l}\text { MEDICINE LAKE } \\
\text { NWR }\end{array}$ & EAST ML LAKE 10 & 2011 & Graze & Graze & Med & 1 & $\{0-30, S B\}$ & Graze \\
\hline
\end{tabular}




\begin{tabular}{|c|c|c|c|c|c|c|c|c|c|c|}
\hline $\begin{array}{l}\text { Grass } \\
\text { Type }\end{array}$ & Complex & Org & Unit & $\begin{array}{c}\text { Management } \\
\text { Year }\end{array}$ & $\begin{array}{c}\text { Management } \\
\text { Applied }\end{array}$ & $\begin{array}{l}\text { Management } \\
\text { Classified }\end{array}$ & $\begin{array}{l}\text { Defoliation } \\
\text { Level }\end{array}$ & $\begin{array}{l}\text { Years Since } \\
\text { Defoliation }\end{array}$ & $\begin{array}{l}\text { Vegetation } \\
\text { State }\end{array}$ & $\begin{array}{c}\text { Management } \\
\text { Recommendation } \\
\text { Year } t+1 \\
\end{array}$ \\
\hline Mixed & $\begin{array}{l}\text { MEDICINE LAKE NWR } \\
\text { COMPLEX }\end{array}$ & $\begin{array}{l}\text { MEDICINE LAKE } \\
\text { NWR }\end{array}$ & EAST ML LAKE 10 & 2012 & Graze & Graze & High & 1 & $\{0-30, S B\}$ & Graze \\
\hline Mixed & $\begin{array}{c}\text { MEDICINE LAKE NWR } \\
\text { COMPLEX }\end{array}$ & $\begin{array}{l}\text { MEDICINE LAKE } \\
\text { NWR }\end{array}$ & $\begin{array}{l}\text { Homestead } \\
\text { North }\end{array}$ & 2010 & Rest & Rest & Low & $2-4$ & $\{60-100, C O\}$ & Burn \\
\hline Mixed & $\begin{array}{l}\text { MEDICINE LAKE NWR } \\
\text { COMPLEX }\end{array}$ & $\begin{array}{l}\text { MEDICINE LAKE } \\
\text { NWR }\end{array}$ & $\begin{array}{l}\text { Homestead } \\
\text { North }\end{array}$ & 2011 & Burn & Burn & Low & 1 & $\{45-60, \mathrm{CO}\}$ & Graze \\
\hline Mixed & $\begin{array}{l}\text { MEDICINE LAKE NWR } \\
\text { COMPLEX }\end{array}$ & $\begin{array}{l}\text { MEDICINE LAKE } \\
\text { NWR }\end{array}$ & $\begin{array}{l}\text { Homestead } \\
\text { North }\end{array}$ & 2012 & Graze & Graze & Med & 1 & $\{45-60, \mathrm{CO}\}$ & Rest \\
\hline Mixed & $\begin{array}{l}\text { MEDICINE LAKE NWR } \\
\text { COMPLEX }\end{array}$ & $\begin{array}{l}\text { MEDICINE LAKE } \\
\text { NWR }\end{array}$ & $\begin{array}{l}\text { Homestead } \\
\text { South }\end{array}$ & 2010 & Rest & Rest & Med & $2-4$ & $\{60-100, S B\}$ & Graze \\
\hline Mixed & $\begin{array}{l}\text { MEDICINE LAKE NWR } \\
\text { COMPLEX }\end{array}$ & $\begin{array}{l}\text { MEDICINE LAKE } \\
\text { NWR }\end{array}$ & $\begin{array}{l}\text { Homestead } \\
\text { South }\end{array}$ & 2011 & Rest & Rest & Low & $2-4$ & $\{45-60, \mathrm{CO}\}$ & Burn/Graze \\
\hline Mixed & $\begin{array}{l}\text { MEDICINE LAKE NWR } \\
\text { COMPLEX }\end{array}$ & $\begin{array}{l}\text { MEDICINE LAKE } \\
\text { NWR }\end{array}$ & $\begin{array}{l}\text { Homestead } \\
\text { South }\end{array}$ & 2012 & Burn/Graze & Burn/Graze & Med & 1 & $\{45-60, \mathrm{CO}\}$ & Rest \\
\hline Mixed & $\begin{array}{l}\text { MEDICINE LAKE NWR } \\
\text { COMPLEX }\end{array}$ & $\begin{array}{c}\text { SHERIDAN COUNY } \\
\text { WPA }\end{array}$ & Anderson & 2010 & Graze & Graze & Med & 1 & $\{45-60, S B\}$ & Graze \\
\hline Mixed & $\begin{array}{c}\text { MEDICINE LAKE NWR } \\
\text { COMPLEX }\end{array}$ & $\begin{array}{l}\text { SHERIDAN COUNY } \\
\text { WPA }\end{array}$ & Anderson & 2011 & Rest & Rest & Med & $2-4$ & $\{30-45, S B\}$ & Graze \\
\hline Mixed & $\begin{array}{l}\text { MEDICINE LAKE NWR } \\
\text { COMPLEX }\end{array}$ & $\begin{array}{c}\text { SHERIDAN COUNY } \\
\text { WPA }\end{array}$ & Anderson & 2012 & Graze & Graze & Med & 1 & $\{30-45, S B\}$ & Graze \\
\hline Mixed & $\begin{array}{c}\text { MEDICINE LAKE NWR } \\
\text { COMPLEX }\end{array}$ & $\begin{array}{l}\text { SHERIDAN COUNY } \\
\text { WPA }\end{array}$ & Gjesdal West & 2010 & Rest & Rest & High & $2-4$ & $\{30-45, S B\}$ & Graze \\
\hline Mixed & $\begin{array}{l}\text { MEDICINE LAKE NWR } \\
\text { COMPLEX }\end{array}$ & $\begin{array}{c}\text { SHERIDAN COUNY } \\
\text { WPA }\end{array}$ & Gjesdal West & 2011 & Graze & Graze & High & 1 & $\{30-45, S B\}$ & Graze \\
\hline Mixed & $\begin{array}{c}\text { MEDICINE LAKE NWR } \\
\text { COMPLEX }\end{array}$ & $\begin{array}{l}\text { SHERIDAN COUNY } \\
\text { WPA }\end{array}$ & Gjesdal West & 2012 & Graze & Graze & High & 1 & $\{0-30, S B\}$ & Graze \\
\hline Mixed & SAND LAKE COMPLEX & $\begin{array}{c}\text { CAMPBELL COUNTY } \\
\text { WPA }\end{array}$ & Cooper North & 2009 & Rest & NA & Low & $2-4$ & $\{30-45, \mathrm{CO}\}$ & NA \\
\hline Mixed & SAND LAKE COMPLEX & $\begin{array}{c}\text { CAMPBELL COUNTY } \\
\text { WPA }\end{array}$ & Cooper North & 2010 & Burn & Burn & Med & 1 & $\{45-60, \mathrm{CO}\}$ & Burn \\
\hline Mixed & SAND LAKE COMPLEX & $\begin{array}{c}\text { CAMPBELL COUNTY } \\
\text { WPA }\end{array}$ & Cooper North & 2011 & Burn & Burn & Med & 1 & $\{45-60, \mathrm{CO}\}$ & Rest \\
\hline
\end{tabular}




\begin{tabular}{|c|c|c|c|c|c|c|c|c|c|c|}
\hline $\begin{array}{l}\text { Grass } \\
\text { Type }\end{array}$ & Complex & Org & Unit & $\begin{array}{c}\text { Management } \\
\text { Year }\end{array}$ & $\begin{array}{c}\text { Management } \\
\text { Applied }\end{array}$ & $\begin{array}{l}\text { Management } \\
\text { Classified }\end{array}$ & $\begin{array}{l}\text { Defoliation } \\
\text { Level }\end{array}$ & $\begin{array}{l}\text { Years Since } \\
\text { Defoliation }\end{array}$ & $\begin{array}{l}\text { Vegetation } \\
\text { State }\end{array}$ & $\begin{array}{c}\text { Management } \\
\text { Recommendation } \\
\text { Year } t+1 \\
\end{array}$ \\
\hline Mixed & SAND LAKE COMPLEX & $\begin{array}{l}\text { CAMPBELL COUNTY } \\
\text { WPA }\end{array}$ & Cooper North & 2012 & Rest & Rest & Med & $2-4$ & $\{30-45, K B\}$ & Burn/Graze \\
\hline Mixed & SAND LAKE COMPLEX & $\begin{array}{c}\text { EDMUNDS COUNTY } \\
\text { WPA }\end{array}$ & Mitzel & 2009 & Rest & NA & Med & $2-4$ & $\{0-30, S B\}$ & NA \\
\hline Mixed & SAND LAKE COMPLEX & $\begin{array}{c}\text { EDMUNDS COUNTY } \\
\text { WPA }\end{array}$ & Mitzel & 2010 & Rest & Rest & Low & $2-4$ & $\{0-30, S B\}$ & Graze \\
\hline Mixed & SAND LAKE COMPLEX & $\begin{array}{c}\text { EDMUNDS COUNTY } \\
\text { WPA }\end{array}$ & Mitzel & 2011 & Graze & Graze & Med & 1 & $\{0-30, \mathrm{CO}\}$ & Rest \\
\hline Mixed & SAND LAKE COMPLEX & $\begin{array}{l}\text { EDMUNDS COUNTY } \\
\text { WPA }\end{array}$ & Mitzel & 2012 & Rest & Rest & Med & $2-4$ & $\{0-30, \mathrm{CO}\}$ & Rest \\
\hline Mixed & SAND LAKE COMPLEX & $\begin{array}{l}\text { MCPHERSON } \\
\text { COUNTY WPA }\end{array}$ & Charley-Harley & 2009 & Rest & NA & Low & $2-4$ & $\{0-30, K B\}$ & NA \\
\hline Mixed & SAND LAKE COMPLEX & $\begin{array}{l}\text { MCPHERSON } \\
\text { COUNTY WPA }\end{array}$ & Charley-Harley & 2010 & Rest & Rest & Low & $5+$ & $\{0-30, K B\}$ & Burn \\
\hline Mixed & SAND LAKE COMPLEX & $\begin{array}{l}\text { MCPHERSON } \\
\text { COUNTY WPA }\end{array}$ & Charley-Harley & 2011 & Burn & Burn & Low & 1 & $\{0-30, K B\}$ & Burn/Graze \\
\hline Mixed & SAND LAKE COMPLEX & $\begin{array}{l}\text { MCPHERSON } \\
\text { COUNTY WPA }\end{array}$ & Charley-Harley & 2012 & Burn & Burn & Med & 1 & $\{30-45, \mathrm{CO}\}$ & Graze \\
\hline Mixed & $\begin{array}{l}\text { SOURIS RIVER BASIN } \\
\text { COMPLEX }\end{array}$ & DES LACS NWR & HB 7 & 2009 & Rest & NA & Low & $2-4$ & $\{0-30, S B\}$ & NA \\
\hline Mixed & $\begin{array}{l}\text { SOURIS RIVER BASIN } \\
\text { COMPLEX }\end{array}$ & DES LACS NWR & HB 7 & 2010 & Burn & Burn & Med & 1 & $\{30-45, S B\}$ & Graze \\
\hline Mixed & $\begin{array}{l}\text { SOURIS RIVER BASIN } \\
\text { COMPLEX }\end{array}$ & DES LACS NWR & HB 7 & 2011 & Rest & Rest & Med & $2-4$ & $\{0-30, K B\}$ & Burn \\
\hline Mixed & $\begin{array}{l}\text { SOURIS RIVER BASIN } \\
\text { COMPLEX }\end{array}$ & DES LACS NWR & HB 7 & 2012 & Burn/Graze & Burn/Graze & Med & 1 & $\{0-30, K B\}$ & Rest \\
\hline Mixed & $\begin{array}{l}\text { SOURIS RIVER BASIN } \\
\text { COMPLEX }\end{array}$ & $\begin{array}{c}\text { J. CLARK SALYER } \\
\text { NWR }\end{array}$ & GLT PLOT A & 2009 & Rest & NA & Med & $2-4$ & $\{45-60, \mathrm{CO}\}$ & NA \\
\hline Mixed & $\begin{array}{l}\text { SOURIS RIVER BASIN } \\
\text { COMPLEX }\end{array}$ & $\begin{array}{c}\text { J. CLARK SALYER } \\
\text { NWR }\end{array}$ & GLT PLOT A & 2010 & Graze & Graze & Med & 1 & $\{45-60, \mathrm{CO}\}$ & Burn \\
\hline Mixed & $\begin{array}{l}\text { SOURIS RIVER BASIN } \\
\text { COMPLEX }\end{array}$ & $\begin{array}{c}\text { J. CLARK SALYER } \\
\text { NWR }\end{array}$ & GLT PLOT A & 2011 & Graze & Graze & Med & 1 & $\{30-45, K B\}$ & Burn \\
\hline
\end{tabular}




\begin{tabular}{|c|c|c|c|c|c|c|c|c|c|c|}
\hline $\begin{array}{l}\text { Grass } \\
\text { Type }\end{array}$ & Complex & Org & Unit & $\begin{array}{c}\text { Management } \\
\text { Year }\end{array}$ & $\begin{array}{c}\text { Management } \\
\text { Applied }\end{array}$ & $\begin{array}{l}\text { Management } \\
\text { Classified }\end{array}$ & $\begin{array}{l}\text { Defoliation } \\
\text { Level }\end{array}$ & $\begin{array}{l}\text { Years Since } \\
\text { Defoliation }\end{array}$ & $\begin{array}{l}\text { Vegetation } \\
\text { State }\end{array}$ & $\begin{array}{c}\text { Management } \\
\text { Recommendation } \\
\text { Year } t+1 \\
\end{array}$ \\
\hline Mixed & $\begin{array}{l}\text { SOURIS RIVER BASIN } \\
\text { COMPLEX }\end{array}$ & $\begin{array}{c}\text { J. CLARK SALYER } \\
\text { NWR }\end{array}$ & GLT PLOT A & 2012 & Burn/Graze & Burn/Graze & Med & 1 & $\{60-100, \mathrm{CO}\}$ & Rest \\
\hline Mixed & $\begin{array}{l}\text { SOURIS RIVER BASIN } \\
\text { COMPLEX }\end{array}$ & $\begin{array}{c}\text { J. CLARK SALYER } \\
\text { NWR }\end{array}$ & GLT PLOT C & 2009 & Rest & NA & Med & $2-4$ & $\{30-45, \mathrm{CO}\}$ & NA \\
\hline Mixed & $\begin{array}{l}\text { SOURIS RIVER BASIN } \\
\text { COMPLEX }\end{array}$ & $\begin{array}{c}\text { J. CLARK SALYER } \\
\text { NWR }\end{array}$ & GLT PLOT C & 2010 & Graze & Graze & Med & 1 & $\{0-30, \mathrm{CO}\}$ & Graze \\
\hline Mixed & $\begin{array}{l}\text { SOURIS RIVER BASIN } \\
\text { COMPLEX }\end{array}$ & $\begin{array}{c}\text { J. CLARK SALYER } \\
\text { NWR }\end{array}$ & GLT PLOT C & 2011 & Graze & Graze & High & 1 & $\{0-30, \mathrm{CO}\}$ & Graze \\
\hline Mixed & $\begin{array}{l}\text { SOURIS RIVER BASIN } \\
\text { COMPLEX }\end{array}$ & $\begin{array}{c}\text { J. CLARK SALYER } \\
\text { NWR }\end{array}$ & GLT PLOT C & 2012 & Burn/Graze & Burn/Graze & High & 1 & $\{30-45, \mathrm{CO}\}$ & Burn/Graze \\
\hline Mixed & $\begin{array}{l}\text { SOURIS RIVER BASIN } \\
\text { COMPLEX }\end{array}$ & $\begin{array}{c}\text { J. CLARK SALYER } \\
\text { NWR }\end{array}$ & Nelson Prairie 1 & 2009 & Rest & NA & Low & $2-4$ & $\{60-100, \mathrm{RM}\}$ & NA \\
\hline Mixed & $\begin{array}{l}\text { SOURIS RIVER BASIN } \\
\text { COMPLEX }\end{array}$ & $\begin{array}{c}\text { J. CLARK SALYER } \\
\text { NWR }\end{array}$ & Nelson Prairie 1 & 2010 & Rest & Rest & Low & $2-4$ & $\{60-100, K B\}$ & Graze \\
\hline Mixed & $\begin{array}{l}\text { SOURIS RIVER BASIN } \\
\text { COMPLEX }\end{array}$ & $\begin{array}{c}\text { J. CLARK SALYER } \\
\text { NWR }\end{array}$ & Nelson Prairie 1 & 2011 & Rest & Rest & Low & $2-4$ & $\{60-100, K B\}$ & Graze \\
\hline Mixed & $\begin{array}{l}\text { SOURIS RIVER BASIN } \\
\text { COMPLEX }\end{array}$ & $\begin{array}{c}\text { J. CLARK SALYER } \\
\text { NWR }\end{array}$ & Nelson Prairie 1 & 2012 & Rest & Rest & Low & $5+$ & $\{60-100, K B\}$ & Burn/Graze \\
\hline Mixed & $\begin{array}{l}\text { SOURIS RIVER BASIN } \\
\text { COMPLEX }\end{array}$ & $\begin{array}{c}\text { J. CLARK SALYER } \\
\text { NWR }\end{array}$ & Nelson Prairie 2 & 2009 & Rest & NA & Low & $2-4$ & $\{60-100, K B\}$ & NA \\
\hline Mixed & $\begin{array}{l}\text { SOURIS RIVER BASIN } \\
\text { COMPLEX }\end{array}$ & $\begin{array}{c}\text { J. CLARK SALYER } \\
\text { NWR }\end{array}$ & Nelson Prairie 2 & 2010 & Rest & Rest & Low & $5+$ & $\{60-100, R M\}$ & Graze \\
\hline Mixed & $\begin{array}{l}\text { SOURIS RIVER BASIN } \\
\text { COMPLEX }\end{array}$ & $\begin{array}{c}\text { J. CLARK SALYER } \\
\text { NWR }\end{array}$ & Nelson Prairie 2 & 2011 & Rest & Rest & Low & $5+$ & $\{60-100, K B\}$ & Burn/Graze \\
\hline Mixed & $\begin{array}{l}\text { SOURIS RIVER BASIN } \\
\text { COMPLEX }\end{array}$ & $\begin{array}{c}\text { J. CLARK SALYER } \\
\text { NWR }\end{array}$ & Nelson Prairie 2 & 2012 & Rest & Rest & Low & $5+$ & $\{45-60, K B\}$ & Burn \\
\hline Mixed & $\begin{array}{l}\text { SOURIS RIVER BASIN } \\
\text { COMPLEX }\end{array}$ & $\begin{array}{c}\text { J. CLARK SALYER } \\
\text { NWR }\end{array}$ & Nelson Prairie 3 & 2009 & Rest & NA & Low & $2-4$ & $\{60-100, K B\}$ & NA \\
\hline Mixed & $\begin{array}{l}\text { SOURIS RIVER BASIN } \\
\text { COMPLEX }\end{array}$ & $\begin{array}{c}\text { J. CLARK SALYER } \\
\text { NWR }\end{array}$ & Nelson Prairie 3 & 2010 & Rest & Rest & Low & $2-4$ & $\{60-100, K B\}$ & Burn \\
\hline Mixed & $\begin{array}{l}\text { SOURIS RIVER BASIN } \\
\text { COMPLEX }\end{array}$ & $\begin{array}{c}\text { J. CLARK SALYER } \\
\text { NWR }\end{array}$ & Nelson Prairie 3 & 2011 & Burn & Burn & Low & 1 & $\{60-100, K B\}$ & Burn/Graze \\
\hline
\end{tabular}




\begin{tabular}{|c|c|c|c|c|c|c|c|c|c|c|}
\hline $\begin{array}{l}\text { Grass } \\
\text { Type }\end{array}$ & Complex & Org & Unit & $\begin{array}{c}\text { Management } \\
\text { Year }\end{array}$ & $\begin{array}{c}\text { Management } \\
\text { Applied }\end{array}$ & $\begin{array}{l}\text { Management } \\
\text { Classified }\end{array}$ & $\begin{array}{l}\text { Defoliation } \\
\text { Level }\end{array}$ & $\begin{array}{l}\text { Years Since } \\
\text { Defoliation }\end{array}$ & $\begin{array}{l}\text { Vegetation } \\
\text { State }\end{array}$ & $\begin{array}{c}\text { Management } \\
\text { Recommendation } \\
\text { Year } t+1 \\
\end{array}$ \\
\hline Mixed & $\begin{array}{l}\text { SOURIS RIVER BASIN } \\
\text { COMPLEX }\end{array}$ & $\begin{array}{c}\text { J. CLARK SALYER } \\
\text { NWR }\end{array}$ & Nelson Prairie 3 & 2012 & Rest & Rest & Low & $2-4$ & $\{60-100, K B\}$ & Graze \\
\hline Mixed & $\begin{array}{l}\text { SOURIS RIVER BASIN } \\
\text { COMPLEX }\end{array}$ & $\begin{array}{c}\text { J. CLARK SALYER } \\
\text { NWR }\end{array}$ & Nelson Prairie 4 & 2009 & Rest & NA & Med & $2-4$ & $\{60-100, K B\}$ & NA \\
\hline Mixed & $\begin{array}{l}\text { SOURIS RIVER BASIN } \\
\text { COMPLEX }\end{array}$ & $\begin{array}{c}\text { J. CLARK SALYER } \\
\text { NWR }\end{array}$ & Nelson Prairie 4 & 2010 & Graze & Graze & Med & 1 & $\{60-100, K B\}$ & Burn \\
\hline Mixed & $\begin{array}{l}\text { SOURIS RIVER BASIN } \\
\text { COMPLEX }\end{array}$ & $\begin{array}{c}\text { J. CLARK SALYER } \\
\text { NWR }\end{array}$ & Nelson Prairie 4 & 2011 & Rest & Rest & Med & $2-4$ & $\{45-60, K B\}$ & Burn \\
\hline Mixed & $\begin{array}{l}\text { SOURIS RIVER BASIN } \\
\text { COMPLEX }\end{array}$ & $\begin{array}{c}\text { J. CLARK SALYER } \\
\text { NWR }\end{array}$ & Nelson Prairie 4 & 2012 & Graze & Graze & High & 1 & $\{45-60, K B\}$ & Rest \\
\hline Mixed & $\begin{array}{l}\text { SOURIS RIVER BASIN } \\
\text { COMPLEX }\end{array}$ & $\begin{array}{c}\text { MCHENRY COUNTY } \\
\text { WPA }\end{array}$ & Keller Unit 1 & 2009 & Graze & NA & High & 1 & $\{60-100, K B\}$ & NA \\
\hline Mixed & $\begin{array}{l}\text { SOURIS RIVER BASIN } \\
\text { COMPLEX }\end{array}$ & $\begin{array}{c}\text { MCHENRY COUNTY } \\
\text { WPA }\end{array}$ & Keller Unit 1 & 2010 & Graze & Graze & High & 1 & $\{60-100, K B\}$ & Burn \\
\hline Mixed & $\begin{array}{l}\text { SOURIS RIVER BASIN } \\
\text { COMPLEX }\end{array}$ & $\begin{array}{c}\text { MCHENRY COUNTY } \\
\text { WPA }\end{array}$ & Keller Unit 1 & 2011 & Graze & Graze & High & 1 & $\{45-60, K B\}$ & Rest \\
\hline Mixed & $\begin{array}{l}\text { SOURIS RIVER BASIN } \\
\text { COMPLEX }\end{array}$ & $\begin{array}{c}\text { MCHENRY COUNTY } \\
\text { WPA }\end{array}$ & Keller Unit 1 & 2012 & Burn/Graze & Burn/Graze & High & 1 & $\{60-100, K B\}$ & Graze \\
\hline Mixed & $\begin{array}{l}\text { SOURIS RIVER BASIN } \\
\text { COMPLEX }\end{array}$ & $\begin{array}{c}\text { MCHENRY COUNTY } \\
\text { WPA }\end{array}$ & Keller Unit 2 & 2009 & Graze & NA & High & 1 & $\{45-60, K B\}$ & NA \\
\hline Mixed & $\begin{array}{l}\text { SOURIS RIVER BASIN } \\
\text { COMPLEX }\end{array}$ & $\begin{array}{c}\text { MCHENRY COUNTY } \\
\text { WPA }\end{array}$ & Keller Unit 2 & 2010 & Rest & Rest & High & $2-4$ & $\{45-60, K B\}$ & Burn \\
\hline Mixed & $\begin{array}{l}\text { SOURIS RIVER BASIN } \\
\text { COMPLEX }\end{array}$ & $\begin{array}{c}\text { MCHENRY COUNTY } \\
\text { WPA }\end{array}$ & Keller Unit 2 & 2011 & Rest & Rest & Med & $2-4$ & $\{30-45, K B\}$ & Burn/Graze \\
\hline Mixed & $\begin{array}{l}\text { SOURIS RIVER BASIN } \\
\text { COMPLEX }\end{array}$ & $\begin{array}{c}\text { MCHENRY COUNTY } \\
\text { WPA }\end{array}$ & Keller Unit 2 & 2012 & Graze & Graze & High & 1 & $\{30-45, K B\}$ & Burn/Graze \\
\hline Mixed & $\begin{array}{l}\text { SOURIS RIVER BASIN } \\
\text { COMPLEX }\end{array}$ & UPPER SOURIS NWR & $\begin{array}{l}\text { HB-24 Ekert } \\
\text { Ranch South }\end{array}$ & 2009 & Rest & NA & Low & $2-4$ & $\{30-45, K B\}$ & NA \\
\hline Mixed & $\begin{array}{l}\text { SOURIS RIVER BASIN } \\
\text { COMPLEX }\end{array}$ & UPPER SOURIS NWR & $\begin{array}{l}\text { HB-24 Ekert } \\
\text { Ranch South }\end{array}$ & 2010 & Burn & Burn & Med & 1 & $\{60-100, K B\}$ & Burn \\
\hline Mixed & $\begin{array}{l}\text { SOURIS RIVER BASIN } \\
\text { COMPLEX }\end{array}$ & UPPER SOURIS NWR & $\begin{array}{l}\text { HB-24 Ekert } \\
\text { Ranch South }\end{array}$ & 2011 & Burn & Burn & Med & 1 & $\{60-100, K B\}$ & Graze \\
\hline
\end{tabular}




\begin{tabular}{|c|c|c|c|c|c|c|c|c|c|c|}
\hline $\begin{array}{l}\text { Grass } \\
\text { Type }\end{array}$ & Complex & Org & Unit & $\begin{array}{c}\text { Management } \\
\text { Year }\end{array}$ & $\begin{array}{c}\text { Management } \\
\text { Applied }\end{array}$ & $\begin{array}{l}\text { Management } \\
\text { Classified }\end{array}$ & $\begin{array}{c}\text { Defoliation } \\
\text { Level }\end{array}$ & $\begin{array}{l}\text { Years Since } \\
\text { Defoliation }\end{array}$ & $\begin{array}{l}\text { Vegetation } \\
\text { State }\end{array}$ & $\begin{array}{c}\text { Management } \\
\text { Recommendation } \\
\text { Year } t+1\end{array}$ \\
\hline Mixed & $\begin{array}{c}\text { SOURIS RIVER BASIN } \\
\text { COMPLEX }\end{array}$ & UPPER SOURIS NWR & $\begin{array}{l}\text { HB-24 Ekert } \\
\text { Ranch South }\end{array}$ & 2012 & Rest & Rest & Med & $2-4$ & $\{45-60, K B\}$ & Burn \\
\hline
\end{tabular}

\section{Field Descriptions}

Management Year: The management year runs from September 1 of the previous calendar year to August 31 of the current calendar year.

For example:

Management Year 2009: September 1, 2008-August 31, 2009

Management Year 2010: September 1, 2009-August 31, 2010

Management Year 2011: September 1, 2010-August 31, 2011

Management Year 2012: September 1, 2011-August 31, 2012

This can be generically written as follows:

Management Year $t$ : September 1, $t$-1-August 31, $t$

Management Applied: The management action that was applied during the management year. This is taken directly from what the cooperator entered into the database as the combination of all management records for a given unit within the management year.

Management Classified: Classification of the applied management into one of the four recognized alternative Native Prairie Adaptive Management (NPAM) actions for mixed-grass units (rest, graze, burn, burn/graze). These classifications are made according to defined NPAM rules. For most mixed-grass units, the 'Management Classified' will be the same as the 'Management Applied'. For the cases where it is not the same, if you have questions, please contact the NPAM Project Coordinator for an explanation specific to your situation. 'Management Classified' for mixed-grass units in 2009 is listed as NA. We did not need to classify the 2009 management because, lacking monitoring data for 2008, we could not use the 2009 management to update model weights.

Defoliation Level: A seven-year window was used to classify the management history of each unit into one of three defoliation categories: Low, Med, or High.

Seven-year windows for each management year: 
2009: From 2003 to 2009

2010: From 2004 to 2010

2011: From 2005 to 2011

2012: From 2006 to 2012

This can be generically written as follows:

$t$ : From $(t-6)$ to $t$

Year $t$, the most recent management year, is included as the closing year of the seven-year window.

Assignment into categories was based on a combination of the years since the last defoliation actoin (graze, burn, burn/graze, or hay) was applied on the unit, and the total number of defoliations (graze, burn, burn/graze, or hay) that were carried out during the entire seven-year window (maximum of one defoliation per management year; that is, a unit was either defoliated or not defoliated in a given year).

Years Since Defoliation: Classifies each unit into one of three 'Years Since Defoliation' levels: 1, 2-4, or 5+.

These classifications denote the number of years it has been since the unit was last defoliated (graze, burn, burn/graze, or hay). A seven-year window was used to determine this state. A ' 1 ' means the unit was defoliated during the most recent management year.

Vegetation State: Vegetation state is defined by the proportions of the four main vegetative components: NP, SB, KB, and RM. $\mathrm{NP}=$ Proportion of the unit that consists of native grasses and forbs (NP). Based on an average of all transects on the unit with monitoring data for the given management year.

$\mathrm{SB}=$ Proportion of the unit that consists of the cool-season invasive species smooth brome (SB). Based on an average of all transects on the unit with monitoring data for the given management year.

$\mathrm{KB}=$ Proportion of the unit that consists of the cool-season invasive species Kentucky bluegrass (KB). Based on an average of all transects on the unit with monitoring data for the given management year.

$\mathrm{RM}=$ Proportion of the unit that consists of vegetation other than NP, SB, or KB. Based on an average of all transects on the unit with monitoring data for the given management year. $\mathrm{RM}=$ remainder.

The Vegetation state is shown as $\{x, y\}$, where: $x=\mathrm{NP}$ level and $y=$ dominant invader (that is, $\mathrm{SB}, \mathrm{KB}, \mathrm{CO}$, or RM).

NP level: The four NP levels are: 0-30 percent, 30-45 percent, 45-60 percent, $60-100$ percent. A unit is classified into one of these four NP levels based on the NP proportion.

Dominant Invader: The dominant invader was determined as follows:

$\mathrm{RM}$ is dominant if: $\mathrm{RM} /(\mathrm{SB}+\mathrm{KB}+\mathrm{RM})>=0.67$

Otherwise, 
$\mathrm{SB}$ is dominant if: $\mathrm{SB} /(\mathrm{SB}+\mathrm{KB})>=0.67$

$\mathrm{KB}$ is dominant if: $\mathrm{KB} /(\mathrm{SB}+\mathrm{KB})>=0.67$

If neither $\mathrm{SB}$ nor $\mathrm{KB}$ is dominant, it is considered a state of co-dominance $(\mathrm{CO})$ by $\mathrm{SB}$ and $\mathrm{KB}$.

Note: Vegetation proportions are calculated excluding the following plant codes: -9 (no data), 24 and 47 (open water, SD and ND list respectively), 91 (barren), and 92 and 99 (other, SD and ND list respectively).

Management Recommendation Year $\boldsymbol{t}+\mathbf{1}$ : Recommended management action for the upcoming management year. The recommendation is based on unit-specific defoliation level, years since defoliation, and vegetation state for the current management year, as well as on the current knowledge state and decision policy for the management year (see appendix 2 for the 2010 policy and figure 13 in the main text for the 2011 and 2012 policy). 
Appendix 8b. Summary of system state and management of tallgrass units from cooperating stations from project inception (2009) through 2012. [The summary includes the grass type, the name [complex and organization (org)] of cooperating stations and the enrolled units, management year, management applied, classification of the applied management, defoliation level, years since last defoliation, and vegetation state, in addition to the management recommendation for the following management year. $\mathrm{SB}=$ smooth brome, $\mathrm{CO}=$ co-dominant smooth brome and Kentucky bluegrass, $\mathrm{KB}=$ Kentucky bluegrass $\mathrm{RM}=$ remainder, and $t=$ current management year. 2011 was the first management year for which management recommendations were provided to cooperators; thus, recommendations for the 2010 management year are documented as NA. Units that do not have data for all four years (2009-12) were either added to the project after its inception or were removed from the project after participating for a time. Following the summary are descriptions of the summary fields]

\begin{tabular}{|c|c|c|c|c|c|c|c|c|c|c|}
\hline $\begin{array}{l}\text { Grass } \\
\text { Type }\end{array}$ & Complex & Org & Unit & $\begin{array}{c}\text { Management } \\
\text { Year }\end{array}$ & $\begin{array}{c}\text { Management } \\
\text { Applied }\end{array}$ & $\begin{array}{c}\text { Management } \\
\text { Classified }\end{array}$ & $\begin{array}{c}\text { Defoliation } \\
\text { Level }\end{array}$ & $\begin{array}{l}\text { Years Since } \\
\text { Defoliation }\end{array}$ & $\begin{array}{l}\text { Vegetation } \\
\text { State }\end{array}$ & $\begin{array}{c}\text { Management } \\
\text { Recommendation } \\
\text { Year } t+1 \\
\end{array}$ \\
\hline Tall & BIG STONE NWR & BIG STONE NWR & Laskowske & 2009 & Burn & NA & Med & 1 & $\{45-60, S B\}$ & NA \\
\hline Tall & BIG STONE NWR & BIG STONE NWR & Laskowske & 2010 & Graze & NA & Med & 1 & $\{60-100, S B\}$ & Graze \\
\hline Tall & BIG STONE NWR & BIG STONE NWR & Laskowske & 2011 & Rest & Rest & Med & $2-4$ & $\{45-60, S B\}$ & $\begin{array}{l}\text { Burn within } \\
\text { window }\end{array}$ \\
\hline Tall & BIG STONE NWR & BIG STONE NWR & Laskowske & 2012 & Rest & Rest & Low & $2-4$ & $\{60-100, S B\}$ & $\begin{array}{l}\text { Burn within } \\
\text { window }\end{array}$ \\
\hline Tall & DETROIT LAKES WMD & $\begin{array}{l}\text { CLAY COUNTY WPA } \\
\text { OF MINNESOTA }\end{array}$ & $\begin{array}{l}\text { Hoykens WPA } \\
\text { North }\end{array}$ & 2009 & Rest & NA & Low & $2-4$ & $\{60-100, K B\}$ & NA \\
\hline Tall & DETROIT LAKES WMD & $\begin{array}{l}\text { CLAY COUNTY WPA } \\
\text { OF MINNESOTA }\end{array}$ & $\begin{array}{l}\text { Hoykens WPA } \\
\text { North }\end{array}$ & 2010 & Rest & NA & Low & $2-4$ & $\{60-100, \mathrm{RM}\}$ & Burn/Graze \\
\hline Tall & DETROIT LAKES WMD & $\begin{array}{l}\text { CLAY COUNTY WPA } \\
\text { OF MINNESOTA }\end{array}$ & $\begin{array}{c}\text { Hoykens WPA } \\
\text { North }\end{array}$ & 2011 & Burn & Defoliate & Low & 1 & $\{60-100, R M\}$ & Defoliate \\
\hline Tall & DETROIT LAKES WMD & $\begin{array}{l}\text { CLAY COUNTY WPA } \\
\text { OF MINNESOTA }\end{array}$ & $\begin{array}{l}\text { Hoykens WPA } \\
\text { North }\end{array}$ & 2012 & Rest & Rest & Low & $2-4$ & $\{60-100, K B\}$ & $\begin{array}{l}\text { Burn within } \\
\text { window }\end{array}$ \\
\hline Tall & DETROIT LAKES WMD & $\begin{array}{l}\text { CLAY COUNTY WPA } \\
\text { OF MINNESOTA }\end{array}$ & Jarvis WPA & 2009 & Rest & NA & Low & $5+$ & $\{30-45, \mathrm{CO}\}$ & NA \\
\hline Tall & DETROIT LAKES WMD & $\begin{array}{l}\text { CLAY COUNTY WPA } \\
\text { OF MINNESOTA }\end{array}$ & Jarvis WPA & 2010 & Burn & NA & Low & 1 & $\{60-100, \mathrm{RM}\}$ & Burn/Graze \\
\hline Tall & DETROIT LAKES WMD & $\begin{array}{l}\text { CLAY COUNTY WPA } \\
\text { OF MINNESOTA }\end{array}$ & Jarvis WPA & 2011 & Rest & Rest & Low & $2-4$ & $\{45-60, S B\}$ & $\begin{array}{l}\text { Burn within } \\
\text { window }\end{array}$ \\
\hline
\end{tabular}




\begin{tabular}{|c|c|c|c|c|c|c|c|c|c|c|}
\hline $\begin{array}{l}\text { Grass } \\
\text { Type }\end{array}$ & Complex & Org & Unit & $\begin{array}{c}\text { Management } \\
\text { Year }\end{array}$ & $\begin{array}{c}\text { Management } \\
\text { Applied }\end{array}$ & $\begin{array}{c}\text { Management } \\
\text { Classified }\end{array}$ & $\begin{array}{c}\text { Defoliation } \\
\text { Level }\end{array}$ & $\begin{array}{l}\text { Years Since } \\
\text { Defoliation }\end{array}$ & $\begin{array}{l}\text { Vegetation } \\
\text { State }\end{array}$ & $\begin{array}{c}\text { Management } \\
\text { Recommendation } \\
\text { Year } t+1\end{array}$ \\
\hline Tall & DETROIT LAKES WMD & $\begin{array}{l}\text { CLAY COUNTY WPA } \\
\text { OF MINNESOTA }\end{array}$ & Jarvis WPA & 2012 & Rest & Rest & Low & $2-4$ & $\{60-100, S B\}$ & $\begin{array}{l}\text { Burn within } \\
\text { window }\end{array}$ \\
\hline Tall & DETROIT LAKES WMD & $\begin{array}{l}\text { MAHNOMEN } \\
\text { COUNTY WPA }\end{array}$ & $\begin{array}{l}\text { Sandy Lake } \\
\text { Native }\end{array}$ & 2010 & Rest & NA & Low & $2-4$ & $\{60-100, C O\}$ & Burn \\
\hline Tall & DETROIT LAKES WMD & $\begin{array}{l}\text { MAHNOMEN } \\
\text { COUNTY WPA }\end{array}$ & $\begin{array}{l}\text { Sandy Lake } \\
\text { Native }\end{array}$ & 2011 & Rest & Rest & Low & $5+$ & $\{0-30, \mathrm{CO}\}$ & $\begin{array}{l}\text { Burn within } \\
\text { window }\end{array}$ \\
\hline Tall & DETROIT LAKES WMD & $\begin{array}{l}\text { MAHNOMEN } \\
\text { COUNTY WPA }\end{array}$ & $\begin{array}{l}\text { Sandy Lake } \\
\text { Native }\end{array}$ & 2012 & Burn & Defoliate & Low & 1 & $\{60-100, S B\}$ & $\begin{array}{l}\text { Burn within } \\
\text { window }\end{array}$ \\
\hline Tall & DEVILS LAKE WMD & $\begin{array}{l}\text { GRAND FORKS } \\
\text { COUNTY WPA }\end{array}$ & Mekinock & 2009 & Rest & NA & Low & $2-4$ & $\{60-100, S B\}$ & NA \\
\hline Tall & DEVILS LAKE WMD & $\begin{array}{l}\text { GRAND FORKS } \\
\text { COUNTY WPA }\end{array}$ & Mekinock & 2010 & Burn & NA & Low & 1 & $\{60-100, S B\}$ & Burn/Graze \\
\hline Tall & DEVILS LAKE WMD & $\begin{array}{l}\text { GRAND FORKS } \\
\text { COUNTY WPA }\end{array}$ & Mekinock & 2011 & Rest & Rest & Low & $2-4$ & $\{60-100, S B\}$ & $\begin{array}{l}\text { Burn within } \\
\text { window }\end{array}$ \\
\hline Tall & DEVILS LAKE WMD & $\begin{array}{l}\text { GRAND FORKS } \\
\text { COUNTY WPA }\end{array}$ & Mekinock & 2012 & Burn & $\begin{array}{l}\text { Burn w/in } \\
\text { window }\end{array}$ & Med & 1 & $\{60-100, \mathrm{RM}\}$ & Defoliate \\
\hline Tall & MADISON WMD & $\begin{array}{l}\text { DEUEL COUNTY } \\
\text { WPA }\end{array}$ & Miller & 2010 & NA & NA & Med & 1 & NA & NA \\
\hline Tall & MADISON WMD & $\begin{array}{l}\text { DEUEL COUNTY } \\
\text { WPA }\end{array}$ & Miller & 2011 & Rest & Rest & Med & $2-4$ & $\{30-45, S B\}$ & $\begin{array}{l}\text { Burn within } \\
\text { window }\end{array}$ \\
\hline Tall & MADISON WMD & $\begin{array}{c}\text { DEUEL COUNTY } \\
\text { WPA }\end{array}$ & Miller & 2012 & Rest & Rest & Med & $2-4$ & $\{45-60, S B\}$ & $\begin{array}{l}\text { Burn within } \\
\text { window }\end{array}$ \\
\hline Tall & MADISON WMD & $\begin{array}{l}\text { HAMLIN COUNTY } \\
\text { WPA }\end{array}$ & Cox 1 & 2009 & Graze & NA & High & 1 & $\{60-100, K B\}$ & NA \\
\hline Tall & MADISON WMD & $\begin{array}{l}\text { HAMLIN COUNTY } \\
\text { WPA }\end{array}$ & Cox 1 & 2010 & Graze & NA & High & 1 & $\{45-60, K B\}$ & Burn \\
\hline Tall & MADISON WMD & $\begin{array}{l}\text { HAMLIN COUNTY } \\
\text { WPA }\end{array}$ & $\operatorname{Cox} 2$ & 2009 & Graze & NA & Med & 1 & $\{0-30, K B\}$ & NA \\
\hline Tall & MADISON WMD & $\begin{array}{l}\text { HAMLIN COUNTY } \\
\text { WPA }\end{array}$ & $\operatorname{Cox} 2$ & 2010 & Burn/Graze & NA & Med & 1 & $\{0-30, \mathrm{CO}\}$ & Burn/Graze \\
\hline Tall & MADISON WMD & $\begin{array}{c}\text { HAMLIN COUNTY } \\
\text { WPA }\end{array}$ & Cox 3 & 2009 & Burn/Graze & NA & High & 1 & $\{30-45, K B\}$ & NA \\
\hline
\end{tabular}




\begin{tabular}{|c|c|c|c|c|c|c|c|c|c|c|}
\hline $\begin{array}{l}\text { Grass } \\
\text { Type }\end{array}$ & Complex & Org & Unit & $\begin{array}{c}\text { Management } \\
\text { Year }\end{array}$ & $\begin{array}{c}\text { Management } \\
\text { Applied }\end{array}$ & $\begin{array}{c}\text { Management } \\
\text { Classified }\end{array}$ & $\begin{array}{c}\text { Defoliation } \\
\text { Level }\end{array}$ & $\begin{array}{l}\text { Years Since } \\
\text { Defoliation }\end{array}$ & $\begin{array}{l}\text { Vegetation } \\
\text { State }\end{array}$ & $\begin{array}{c}\text { Management } \\
\text { Recommendation } \\
\text { Year } t+1\end{array}$ \\
\hline Tall & MADISON WMD & $\begin{array}{c}\text { HAMLIN COUNTY } \\
\text { WPA }\end{array}$ & $\operatorname{Cox} 3$ & 2010 & Graze & NA & High & 1 & $\{45-60, \mathrm{~KB}\}$ & Burn \\
\hline Tall & MADISON WMD & $\begin{array}{l}\text { MINER COUNTY } \\
\text { WPA }\end{array}$ & Hepner WPA & 2010 & NA & NA & Low & $2-4$ & NA & NA \\
\hline Tall & MADISON WMD & $\begin{array}{l}\text { MINER COUNTY } \\
\text { WPA }\end{array}$ & Hepner WPA & 2011 & Rest & Rest & Low & $2-4$ & $\{0-30, \mathrm{CO}\}$ & $\begin{array}{c}\text { Graze within } \\
\text { window }\end{array}$ \\
\hline Tall & MADISON WMD & $\begin{array}{l}\text { MINER COUNTY } \\
\text { WPA }\end{array}$ & Hepner WPA & 2012 & Graze & $\begin{array}{l}\text { Graze w/in } \\
\text { window }\end{array}$ & Med & 1 & $\{0-30, \mathrm{CO}\}$ & $\begin{array}{l}\text { Burn within } \\
\text { window }\end{array}$ \\
\hline Tall & MADISON WMD & $\begin{array}{l}\text { MINNEHAHA } \\
\text { COUNTY WPA }\end{array}$ & Buffalo Lake 80 & 2010 & NA & NA & Low & $2-4$ & NA & NA \\
\hline Tall & MADISON WMD & $\begin{array}{l}\text { MINNEHAHA } \\
\text { COUNTY WPA }\end{array}$ & Buffalo Lake 80 & 2011 & Graze & Defoliate & Med & 1 & $\{0-30, \mathrm{CO}\}$ & $\begin{array}{l}\text { Burn within } \\
\text { window }\end{array}$ \\
\hline Tall & MADISON WMD & $\begin{array}{l}\text { MINNEHAHA } \\
\text { COUNTY WPA }\end{array}$ & Buffalo Lake 80 & 2012 & Rest & Rest & Med & $2-4$ & $\{0-30, S B\}$ & $\begin{array}{l}\text { Burn within } \\
\text { window }\end{array}$ \\
\hline Tall & MORRIS WMD & $\begin{array}{c}\text { BIG STONE COUNTY } \\
\text { WPA }\end{array}$ & Hillman A & 2009 & Rest & NA & Med & $2-4$ & $\{45-60, K B\}$ & NA \\
\hline Tall & MORRIS WMD & $\begin{array}{c}\text { BIG STONE COUNTY } \\
\text { WPA }\end{array}$ & Hillman A & 2010 & Burn & NA & Med & 1 & $\{60-100, K B\}$ & Burn \\
\hline Tall & MORRIS WMD & $\begin{array}{c}\text { BIG STONE COUNTY } \\
\text { WPA }\end{array}$ & Hillman A & 2011 & Rest & Rest & Med & $2-4$ & $\{45-60, \mathrm{RM}\}$ & $\begin{array}{c}\text { Graze within } \\
\text { window }\end{array}$ \\
\hline Tall & MORRIS WMD & $\begin{array}{c}\text { BIG STONE COUNTY } \\
\text { WPA }\end{array}$ & Hillman A & 2012 & Rest & Rest & Med & $2-4$ & $\{60-100, K B\}$ & $\begin{array}{l}\text { Burn within } \\
\text { window }\end{array}$ \\
\hline Tall & MORRIS WMD & $\begin{array}{c}\text { BIG STONE COUNTY } \\
\text { WPA }\end{array}$ & Hillman B & 2009 & Burn & NA & Low & 1 & $\{30-45, \mathrm{CO}\}$ & NA \\
\hline Tall & MORRIS WMD & $\begin{array}{c}\text { BIG STONE COUNTY } \\
\text { WPA }\end{array}$ & Hillman B & 2010 & Rest & NA & Low & $2-4$ & $\{45-60, \mathrm{CO}\}$ & Burn \\
\hline Tall & MORRIS WMD & $\begin{array}{c}\text { BIG STONE COUNTY } \\
\text { WPA }\end{array}$ & Hillman B & 2011 & Graze & $\begin{array}{c}\text { Graze w/in } \\
\text { window }\end{array}$ & Med & 1 & $\{60-100, \mathrm{CO}\}$ & $\begin{array}{l}\text { Burn within } \\
\text { window }\end{array}$ \\
\hline Tall & MORRIS WMD & $\begin{array}{c}\text { BIG STONE COUNTY } \\
\text { WPA }\end{array}$ & Hillman B & 2012 & Graze & Defoliate & Med & 1 & $\{45-60, \mathrm{CO}\}$ & $\begin{array}{l}\text { Burn within } \\
\text { window }\end{array}$ \\
\hline Tall & MORRIS WMD & $\begin{array}{c}\text { BIG STONE COUNTY } \\
\text { WPA }\end{array}$ & Hillman C & 2009 & Hay & NA & Low & 1 & NA & NA \\
\hline
\end{tabular}




\begin{tabular}{|c|c|c|c|c|c|c|c|c|c|c|}
\hline $\begin{array}{l}\text { Grass } \\
\text { Type }\end{array}$ & Complex & Org & Unit & $\begin{array}{c}\text { Management } \\
\text { Year }\end{array}$ & $\begin{array}{c}\text { Management } \\
\text { Applied }\end{array}$ & $\begin{array}{l}\text { Management } \\
\text { Classified }\end{array}$ & $\begin{array}{c}\text { Defoliation } \\
\text { Level }\end{array}$ & $\begin{array}{l}\text { Years Since } \\
\text { Defoliation }\end{array}$ & $\begin{array}{l}\text { Vegetation } \\
\text { State }\end{array}$ & $\begin{array}{c}\text { Management } \\
\text { Recommendation } \\
\text { Year } t+1\end{array}$ \\
\hline Tall & MORRIS WMD & $\begin{array}{c}\text { BIG STONE COUNTY } \\
\text { WPA }\end{array}$ & Hillman C & 2010 & Rest & NA & Low & $2-4$ & $\{0-30, K B\}$ & Graze \\
\hline Tall & MORRIS WMD & $\begin{array}{c}\text { BIG STONE COUNTY } \\
\text { WPA }\end{array}$ & Hillman C & 2011 & Graze & Defoliate & Med & 1 & $\{0-30, K B\}$ & $\begin{array}{l}\text { Burn within } \\
\text { window }\end{array}$ \\
\hline Tall & MORRIS WMD & $\begin{array}{c}\text { BIG STONE COUNTY } \\
\text { WPA }\end{array}$ & Hillman D & 2009 & Graze & NA & Med & 1 & $\{60-100, K B\}$ & NA \\
\hline Tall & MORRIS WMD & $\begin{array}{c}\text { BIG STONE COUNTY } \\
\text { WPA }\end{array}$ & Hillman D & 2010 & Graze & NA & Med & 1 & $\{60-100, K B\}$ & Burn \\
\hline Tall & MORRIS WMD & $\begin{array}{c}\text { BIG STONE COUNTY } \\
\text { WPA }\end{array}$ & Hillman D & 2011 & Burn & $\begin{array}{l}\text { Burn w/in } \\
\text { window }\end{array}$ & Med & 1 & $\{60-100, K B\}$ & $\begin{array}{l}\text { Burn within } \\
\text { window }\end{array}$ \\
\hline Tall & MORRIS WMD & $\begin{array}{c}\text { BIG STONE COUNTY } \\
\text { WPA }\end{array}$ & Hillman D & 2012 & Rest & Rest & Med & $2-4$ & $\{60-100, K B\}$ & $\begin{array}{l}\text { Burn within } \\
\text { window }\end{array}$ \\
\hline Tall & MORRIS WMD & $\begin{array}{l}\text { LAC QUI PARLE } \\
\text { COUNTY WPA }\end{array}$ & Florida Creek A & 2009 & Rest & NA & Low & $2-4$ & $\{60-100, K B\}$ & NA \\
\hline Tall & MORRIS WMD & $\begin{array}{l}\text { LAC QUI PARLE } \\
\text { COUNTY WPA }\end{array}$ & Florida Creek A & 2010 & Burn & NA & Low & 1 & $\{60-100, K B\}$ & Burn \\
\hline Tall & MORRIS WMD & $\begin{array}{l}\text { LAC QUI PARLE } \\
\text { COUNTY WPA }\end{array}$ & Florida Creek A & 2011 & Rest & Rest & Low & $2-4$ & $\{60-100, R M\}$ & Defoliate \\
\hline Tall & MORRIS WMD & $\begin{array}{l}\text { LAC QUI PARLE } \\
\text { COUNTY WPA }\end{array}$ & Florida Creek A & 2012 & Hay & Defoliate & Med & 1 & $\{60-100, K B\}$ & $\begin{array}{l}\text { Burn within } \\
\text { window }\end{array}$ \\
\hline Tall & MORRIS WMD & $\begin{array}{l}\text { LAC QUI PARLE } \\
\text { COUNTY WPA }\end{array}$ & Florida Creek B & 2009 & Rest & NA & Low & $5+$ & $\{0-30, S B\}$ & NA \\
\hline Tall & MORRIS WMD & $\begin{array}{l}\text { LAC QUI PARLE } \\
\text { COUNTY WPA }\end{array}$ & Florida Creek B & 2010 & Burn & NA & Low & 1 & $\{0-30, S B\}$ & Graze \\
\hline Tall & MORRIS WMD & $\begin{array}{l}\text { LAC QUI PARLE } \\
\text { COUNTY WPA }\end{array}$ & Florida Creek B & 2011 & Rest & Rest & Low & $2-4$ & $\{30-45, \mathrm{SB}\}$ & $\begin{array}{l}\text { Burn within } \\
\text { window }\end{array}$ \\
\hline Tall & MORRIS WMD & $\begin{array}{l}\text { LAC QUI PARLE } \\
\text { COUNTY WPA }\end{array}$ & Florida Creek B & 2012 & Burn & $\begin{array}{l}\text { Burn w/in } \\
\text { window }\end{array}$ & Low & 1 & $\{45-60, S B\}$ & $\begin{array}{c}\text { Graze within } \\
\text { window }\end{array}$ \\
\hline Tall & MORRIS WMD & $\begin{array}{l}\text { LAC QUI PARLE } \\
\text { COUNTY WPA }\end{array}$ & Florida Creek C & 2009 & Rest & NA & Low & $2-4$ & $\{60-100, \mathrm{RM}\}$ & NA \\
\hline Tall & MORRIS WMD & $\begin{array}{l}\text { LAC QUI PARLE } \\
\text { COUNTY WPA }\end{array}$ & Florida Creek C & 2010 & Burn & NA & Med & 1 & $\{60-100, R M\}$ & Burn/Graze \\
\hline
\end{tabular}




\begin{tabular}{|c|c|c|c|c|c|c|c|c|c|c|}
\hline $\begin{array}{l}\text { Grass } \\
\text { Type }\end{array}$ & Complex & Org & Unit & $\begin{array}{c}\text { Management } \\
\text { Year }\end{array}$ & $\begin{array}{c}\text { Management } \\
\text { Applied }\end{array}$ & $\begin{array}{l}\text { Management } \\
\text { Classified }\end{array}$ & $\begin{array}{c}\text { Defoliation } \\
\text { Level }\end{array}$ & $\begin{array}{l}\text { Years Since } \\
\text { Defoliation }\end{array}$ & $\begin{array}{l}\text { Vegetation } \\
\text { State }\end{array}$ & $\begin{array}{c}\text { Management } \\
\text { Recommendation } \\
\text { Year } t+1\end{array}$ \\
\hline Tall & MORRIS WMD & $\begin{array}{l}\text { LAC QUI PARLE } \\
\text { COUNTY WPA }\end{array}$ & Florida Creek C & 2011 & Rest & Rest & Low & $2-4$ & $\{60-100, \mathrm{RM}\}$ & Defoliate \\
\hline Tall & MORRIS WMD & $\begin{array}{l}\text { LAC QUI PARLE } \\
\text { COUNTY WPA }\end{array}$ & Florida Creek C & 2012 & Hay & Defoliate & Med & 1 & $\{60-100, \mathrm{RM}\}$ & Defoliate \\
\hline Tall & MORRIS WMD & $\begin{array}{l}\text { LAC QUI PARLE } \\
\text { COUNTY WPA }\end{array}$ & Freeland A & 2009 & Rest & NA & Low & $2-4$ & $\{0-30, K B\}$ & NA \\
\hline Tall & MORRIS WMD & $\begin{array}{l}\text { LAC QUI PARLE } \\
\text { COUNTY WPA }\end{array}$ & Freeland A & 2010 & Burn & NA & Med & 1 & $\{0-30, K B\}$ & Burn/Graze \\
\hline Tall & MORRIS WMD & $\begin{array}{l}\text { LAC QUI PARLE } \\
\text { COUNTY WPA }\end{array}$ & Freeland A & 2011 & Graze & $\begin{array}{c}\text { Graze w/in } \\
\text { window }\end{array}$ & Med & 1 & $\{0-30, R M\}$ & $\begin{array}{l}\text { Graze within } \\
\text { window }\end{array}$ \\
\hline Tall & MORRIS WMD & $\begin{array}{l}\text { LAC QUI PARLE } \\
\text { COUNTY WPA }\end{array}$ & Freeland A & 2012 & Graze & Unclassifiable & Med & 1 & $\{0-30, \mathrm{RM}\}$ & $\begin{array}{l}\text { Graze within } \\
\text { window }\end{array}$ \\
\hline Tall & MORRIS WMD & $\begin{array}{l}\text { LAC QUI PARLE } \\
\text { COUNTY WPA }\end{array}$ & Freeland B & 2009 & Rest & NA & Med & $2-4$ & $\{30-45, \mathrm{CO}\}$ & NA \\
\hline Tall & MORRIS WMD & $\begin{array}{l}\text { LAC QUI PARLE } \\
\text { COUNTY WPA }\end{array}$ & Freeland B & 2010 & Burn & NA & Med & 1 & $\{0-30, \mathrm{RM}\}$ & Graze \\
\hline Tall & MORRIS WMD & $\begin{array}{l}\text { LAC QUI PARLE } \\
\text { COUNTY WPA }\end{array}$ & Freeland B & 2011 & Graze & $\begin{array}{c}\text { Graze w/in } \\
\text { window }\end{array}$ & High & 1 & $\{45-60, \mathrm{RM}\}$ & $\begin{array}{c}\text { Graze within } \\
\text { window }\end{array}$ \\
\hline Tall & MORRIS WMD & $\begin{array}{l}\text { LAC QUI PARLE } \\
\text { COUNTY WPA }\end{array}$ & Freeland B & 2012 & Graze & Unclassifiable & High & 1 & $\{30-45, \mathrm{RM}\}$ & $\begin{array}{l}\text { Graze within } \\
\text { window }\end{array}$ \\
\hline Tall & SAND LAKE COMPLEX & $\begin{array}{c}\text { BROWN COUNTY } \\
\text { WPA }\end{array}$ & Hayes & 2009 & Rest & NA & Low & $5+$ & $\{30-45, \mathrm{SB}\}$ & NA \\
\hline Tall & SAND LAKE COMPLEX & $\begin{array}{c}\text { BROWN COUNTY } \\
\text { WPA }\end{array}$ & Hayes & 2010 & Rest & NA & Low & $5+$ & $\{30-45, \mathrm{CO}\}$ & Burn \\
\hline Tall & SAND LAKE COMPLEX & $\begin{array}{l}\text { SPINK COUNTY } \\
\text { WPA }\end{array}$ & Sanderson & 2009 & Rest & NA & Low & $5+$ & $\{0-30, S B\}$ & NA \\
\hline Tall & SAND LAKE COMPLEX & $\begin{array}{c}\text { SPINK COUNTY } \\
\text { WPA }\end{array}$ & Sanderson & 2010 & Rest & NA & Low & $5+$ & $\{0-30, \mathrm{CO}\}$ & Burn \\
\hline Tall & SAND LAKE COMPLEX & $\begin{array}{l}\text { SPINK COUNTY } \\
\text { WPA }\end{array}$ & Sanderson & 2011 & Burn & Unclassifiable & Low & 1 & $\{30-45, \mathrm{CO}\}$ & $\begin{array}{l}\text { Burn within } \\
\text { window }\end{array}$ \\
\hline Tall & SAND LAKE COMPLEX & $\begin{array}{c}\text { SPINK COUNTY } \\
\text { WPA }\end{array}$ & Sanderson & 2012 & Burn & Defoliate & Low & 1 & $\{0-30, S B\}$ & $\begin{array}{l}\text { Graze within } \\
\text { window }\end{array}$ \\
\hline
\end{tabular}




\begin{tabular}{|c|c|c|c|c|c|c|c|c|c|c|}
\hline $\begin{array}{l}\text { Grass } \\
\text { Type }\end{array}$ & Complex & Org & Unit & $\begin{array}{c}\text { Management } \\
\text { Year }\end{array}$ & $\begin{array}{c}\text { Management } \\
\text { Applied }\end{array}$ & $\begin{array}{l}\text { Management } \\
\text { Classified }\end{array}$ & $\begin{array}{c}\text { Defoliation } \\
\text { Level }\end{array}$ & $\begin{array}{l}\text { Years Since } \\
\text { Defoliation }\end{array}$ & $\begin{array}{l}\text { Vegetation } \\
\text { State }\end{array}$ & $\begin{array}{c}\text { Management } \\
\text { Recommendation } \\
\text { Year } t+1\end{array}$ \\
\hline Tall & TEWAUKON WMD & $\begin{array}{c}\text { RICHLAND COUNTY } \\
\text { WPA }\end{array}$ & Hartleben Unit A & 2009 & Rest & NA & Low & $5+$ & $\{60-100, \mathrm{RM}\}$ & NA \\
\hline Tall & TEWAUKON WMD & $\begin{array}{c}\text { RICHLAND COUNTY } \\
\text { WPA }\end{array}$ & Hartleben Unit A & 2010 & Rest & NA & Low & $5+$ & $\{60-100, \mathrm{RM}\}$ & Rest \\
\hline Tall & TEWAUKON WMD & $\begin{array}{c}\text { RICHLAND COUNTY } \\
\text { WPA }\end{array}$ & Hartleben Unit A & 2011 & Rest & Rest & Low & $5+$ & $\{60-100, K B\}$ & $\begin{array}{l}\text { Burn within } \\
\text { window }\end{array}$ \\
\hline Tall & TEWAUKON WMD & $\begin{array}{c}\text { RICHLAND COUNTY } \\
\text { WPA }\end{array}$ & Hartleben Unit A & 2012 & Rest & Rest & Low & $5+$ & $\{60-100, R M\}$ & Defoliate \\
\hline Tall & TEWAUKON WMD & $\begin{array}{c}\text { RICHLAND COUNTY } \\
\text { WPA }\end{array}$ & Hartleben Unit B & 2009 & Rest & NA & Low & $5+$ & $\{45-60, S B\}$ & NA \\
\hline Tall & TEWAUKON WMD & $\begin{array}{c}\text { RICHLAND COUNTY } \\
\text { WPA }\end{array}$ & Hartleben Unit B & 2010 & Rest & NA & Low & $5+$ & $\{45-60, S B\}$ & Graze \\
\hline Tall & TEWAUKON WMD & $\begin{array}{c}\text { RICHLAND COUNTY } \\
\text { WPA }\end{array}$ & Hartleben Unit B & 2011 & Rest & Rest & Low & $5+$ & $\{45-60, S B\}$ & $\begin{array}{l}\text { Burn within } \\
\text { window }\end{array}$ \\
\hline Tall & TEWAUKON WMD & $\begin{array}{c}\text { RICHLAND COUNTY } \\
\text { WPA }\end{array}$ & Hartleben Unit B & 2012 & Burn & Unclassifiable & Low & 1 & $\{45-60, S B\}$ & $\begin{array}{c}\text { Graze within } \\
\text { window }\end{array}$ \\
\hline Tall & TEWAUKON WMD & $\begin{array}{c}\text { RICHLAND COUNTY } \\
\text { WPA }\end{array}$ & Hartleben Unit C & 2009 & Rest & NA & Low & $5+$ & $\{60-100, S B\}$ & NA \\
\hline Tall & TEWAUKON WMD & $\begin{array}{c}\text { RICHLAND COUNTY } \\
\text { WPA }\end{array}$ & Hartleben Unit C & 2010 & Rest & NA & Low & $5+$ & $\{60-100, \mathrm{CO}\}$ & Burn \\
\hline Tall & TEWAUKON WMD & $\begin{array}{c}\text { RICHLAND COUNTY } \\
\text { WPA }\end{array}$ & Hartleben Unit C & 2011 & Rest & Rest & Low & $5+$ & $\{60-100, S B\}$ & $\begin{array}{c}\text { Graze within } \\
\text { window }\end{array}$ \\
\hline Tall & TEWAUKON WMD & $\begin{array}{c}\text { RICHLAND COUNTY } \\
\text { WPA }\end{array}$ & Hartleben Unit C & 2012 & Burn & Unclassifiable & Low & 1 & $\{60-100, S B\}$ & $\begin{array}{c}\text { Graze within } \\
\text { window }\end{array}$ \\
\hline Tall & TEWAUKON WMD & $\begin{array}{c}\text { SARGENT COUNTY } \\
\text { WPA }\end{array}$ & Gainor Unit A & 2009 & Rest & NA & High & $2-4$ & $\{0-30, K B\}$ & NA \\
\hline Tall & TEWAUKON WMD & $\begin{array}{c}\text { SARGENT COUNTY } \\
\text { WPA }\end{array}$ & Gainor Unit A & 2010 & Rest & NA & Med & $2-4$ & $\{0-30, K B\}$ & Burn/Graze \\
\hline Tall & TEWAUKON WMD & $\begin{array}{c}\text { SARGENT COUNTY } \\
\text { WPA }\end{array}$ & Gainor Unit A & 2011 & Graze & Defoliate & High & 1 & $\{30-45, \mathrm{~KB}\}$ & $\begin{array}{l}\text { Burn within } \\
\text { window }\end{array}$ \\
\hline Tall & TEWAUKON WMD & $\begin{array}{c}\text { SARGENT COUNTY } \\
\text { WPA }\end{array}$ & Gainor Unit A & 2012 & Rest & Rest & Med & $2-4$ & $\{30-45, K B\}$ & $\begin{array}{l}\text { Burn within } \\
\text { window }\end{array}$ \\
\hline
\end{tabular}




\begin{tabular}{|c|c|c|c|c|c|c|c|c|c|c|}
\hline $\begin{array}{l}\text { Grass } \\
\text { Type }\end{array}$ & Complex & Org & Unit & $\begin{array}{c}\text { Management } \\
\text { Year }\end{array}$ & $\begin{array}{c}\text { Management } \\
\text { Applied }\end{array}$ & $\begin{array}{c}\text { Management } \\
\text { Classified }\end{array}$ & $\begin{array}{c}\text { Defoliation } \\
\text { Level }\end{array}$ & $\begin{array}{l}\text { Years Since } \\
\text { Defoliation }\end{array}$ & $\begin{array}{l}\text { Vegetation } \\
\text { State }\end{array}$ & $\begin{array}{c}\text { Management } \\
\text { Recommendation } \\
\text { Year } t+1\end{array}$ \\
\hline Tall & TEWAUKON WMD & $\begin{array}{c}\text { SARGENT COUNTY } \\
\text { WPA }\end{array}$ & Gainor Unit B & 2009 & Rest & NA & High & $2-4$ & $\{45-60, \mathrm{CO}\}$ & NA \\
\hline Tall & TEWAUKON WMD & $\begin{array}{c}\text { SARGENT COUNTY } \\
\text { WPA }\end{array}$ & Gainor Unit B & 2010 & Graze & NA & High & 1 & $\{45-60, K B\}$ & Graze \\
\hline Tall & TEWAUKON WMD & $\begin{array}{c}\text { SARGENT COUNTY } \\
\text { WPA }\end{array}$ & Gainor Unit B & 2011 & Graze & Defoliate & High & 1 & $\{60-100, R M\}$ & Rest \\
\hline Tall & TEWAUKON WMD & $\begin{array}{c}\text { SARGENT COUNTY } \\
\text { WPA }\end{array}$ & Gainor Unit B & 2012 & Rest & Rest & High & $2-4$ & $\{60-100, R M\}$ & Defoliate \\
\hline Tall & TEWAUKON WMD & $\begin{array}{c}\text { SARGENT COUNTY } \\
\text { WPA }\end{array}$ & Krause & 2009 & Rest & NA & High & $2-4$ & $\{0-30, K B\}$ & NA \\
\hline Tall & TEWAUKON WMD & $\begin{array}{c}\text { SARGENT COUNTY } \\
\text { WPA }\end{array}$ & Krause & 2010 & Burn & NA & High & 1 & $\{0-30, K B\}$ & Burn/Graze \\
\hline Tall & TEWAUKON WMD & $\begin{array}{c}\text { SARGENT COUNTY } \\
\text { WPA }\end{array}$ & Krause & 2011 & Graze & Defoliate & High & 1 & $\{0-30, K B\}$ & $\begin{array}{l}\text { Burn within } \\
\text { window }\end{array}$ \\
\hline Tall & TEWAUKON WMD & $\begin{array}{c}\text { SARGENT COUNTY } \\
\text { WPA }\end{array}$ & Krause & 2012 & Burn & Defoliate & High & 1 & $\{0-30, K B\}$ & $\begin{array}{l}\text { Burn within } \\
\text { window }\end{array}$ \\
\hline Tall & TEWAUKON WMD & TEWAUKON NWR & Tewaukon NWR & 2009 & Rest & NA & Low & $5+$ & $\{0-30, K B\}$ & NA \\
\hline Tall & TEWAUKON WMD & TEWAUKON NWR & Tewaukon NWR & 2010 & Graze & NA & Low & 1 & $\{0-30, K B\}$ & Burn/Graze \\
\hline Tall & TEWAUKON WMD & TEWAUKON NWR & Tewaukon NWR & 2011 & Burn/Graze & Defoliate & Low & 1 & $\{0-30, K B\}$ & $\begin{array}{l}\text { Burn within } \\
\text { window }\end{array}$ \\
\hline Tall & TEWAUKON WMD & TEWAUKON NWR & Tewaukon NWR & 2012 & Rest & Rest & Low & $2-4$ & $\{0-30, K B\}$ & $\begin{array}{l}\text { Burn within } \\
\text { window }\end{array}$ \\
\hline Tall & $\begin{array}{l}\text { WAUBAY NWR } \\
\text { COMPLEX }\end{array}$ & $\begin{array}{c}\text { CLARK COUNTY } \\
\text { WPA }\end{array}$ & $\begin{array}{c}\text { Warner Lake } \\
\text { Paddock } 5\end{array}$ & 2010 & Rest & NA & Low & $2-4$ & $\{0-30, \mathrm{CO}\}$ & Burn/Graze \\
\hline Tall & $\begin{array}{l}\text { WAUBAY NWR } \\
\text { COMPLEX }\end{array}$ & $\begin{array}{c}\text { CLARK COUNTY } \\
\text { WPA }\end{array}$ & $\begin{array}{c}\text { Warner Lake } \\
\text { Paddock } 5\end{array}$ & 2011 & Burn/Graze & Defoliate & Low & 1 & $\{0-30, \mathrm{CO}\}$ & $\begin{array}{l}\text { Graze within } \\
\text { window }\end{array}$ \\
\hline Tall & $\begin{array}{l}\text { WAUBAY NWR } \\
\text { COMPLEX }\end{array}$ & $\begin{array}{c}\text { CLARK COUNTY } \\
\text { WPA }\end{array}$ & $\begin{array}{c}\text { Warner Lake } \\
\text { Paddock } 5\end{array}$ & 2012 & Graze & $\begin{array}{l}\text { Graze w/in } \\
\text { window }\end{array}$ & Med & 1 & $\{0-30, \mathrm{CO}\}$ & $\begin{array}{l}\text { Burn within } \\
\text { window }\end{array}$ \\
\hline Tall & $\begin{array}{l}\text { WAUBAY NWR } \\
\text { COMPLEX }\end{array}$ & $\begin{array}{l}\text { CODINGTON } \\
\text { COUNTY WPA }\end{array}$ & Roe $\mathrm{E}$ & 2009 & Rest & NA & Low & $2-4$ & $\{0-30, S B\}$ & NA \\
\hline
\end{tabular}




\begin{tabular}{|c|c|c|c|c|c|c|c|c|c|c|}
\hline $\begin{array}{l}\text { Grass } \\
\text { Type }\end{array}$ & Complex & Org & Unit & $\begin{array}{c}\text { Management } \\
\text { Year }\end{array}$ & $\begin{array}{c}\text { Management } \\
\text { Applied }\end{array}$ & $\begin{array}{c}\text { Management } \\
\text { Classified }\end{array}$ & $\begin{array}{c}\text { Defoliation } \\
\text { Level }\end{array}$ & $\begin{array}{l}\text { Years Since } \\
\text { Defoliation }\end{array}$ & $\begin{array}{l}\text { Vegetation } \\
\text { State }\end{array}$ & $\begin{array}{c}\text { Management } \\
\text { Recommendation } \\
\text { Year } t+1\end{array}$ \\
\hline Tall & $\begin{array}{l}\text { WAUBAY NWR } \\
\text { COMPLEX }\end{array}$ & $\begin{array}{l}\text { CODINGTON } \\
\text { COUNTY WPA }\end{array}$ & Roe $\mathrm{E}$ & 2010 & Rest & NA & Low & $5+$ & $\{0-30, \mathrm{CO}\}$ & Burn/Graze \\
\hline Tall & $\begin{array}{l}\text { WAUBAY NWR } \\
\text { COMPLEX }\end{array}$ & $\begin{array}{l}\text { CODINGTON } \\
\text { COUNTY WPA }\end{array}$ & Roe $\mathrm{E}$ & 2011 & Burn/Graze & Unclassifiable & Med & 1 & $\{0-30, S B\}$ & $\begin{array}{c}\text { Graze within } \\
\text { window }\end{array}$ \\
\hline Tall & $\begin{array}{l}\text { WAUBAY NWR } \\
\text { COMPLEX }\end{array}$ & $\begin{array}{l}\text { CODINGTON } \\
\text { COUNTY WPA }\end{array}$ & Roe $\mathrm{E}$ & 2012 & Graze & $\begin{array}{c}\text { Graze w/in } \\
\text { window }\end{array}$ & Med & 1 & $\{0-30, \mathrm{CO}\}$ & $\begin{array}{l}\text { Burn within } \\
\text { window }\end{array}$ \\
\hline Tall & $\begin{array}{l}\text { WAUBAY NWR } \\
\text { COMPLEX }\end{array}$ & $\begin{array}{l}\text { CODINGTON } \\
\text { COUNTY WPA }\end{array}$ & Roe $F$ & 2009 & Rest & NA & Low & $2-4$ & $\{0-30, S B\}$ & NA \\
\hline Tall & $\begin{array}{l}\text { WAUBAY NWR } \\
\text { COMPLEX }\end{array}$ & $\begin{array}{l}\text { CODINGTON } \\
\text { COUNTY WPA }\end{array}$ & Roe $F$ & 2010 & Rest & NA & Low & $2-4$ & $\{0-30, \mathrm{CO}\}$ & Burn/Graze \\
\hline Tall & $\begin{array}{l}\text { WAUBAY NWR } \\
\text { COMPLEX }\end{array}$ & $\begin{array}{l}\text { CODINGTON } \\
\text { COUNTY WPA }\end{array}$ & Roe F & 2011 & Burn/Graze & Unclassifiable & Med & 1 & $\{0-30, K B\}$ & $\begin{array}{l}\text { Burn within } \\
\text { window }\end{array}$ \\
\hline Tall & $\begin{array}{l}\text { WAUBAY NWR } \\
\text { COMPLEX }\end{array}$ & $\begin{array}{l}\text { CODINGTON } \\
\text { COUNTY WPA }\end{array}$ & Roe $F$ & 2012 & Burn & $\begin{array}{l}\text { Burn w/in } \\
\text { window }\end{array}$ & Med & 1 & $\{0-30, \mathrm{CO}\}$ & $\begin{array}{l}\text { Burn within } \\
\text { window }\end{array}$ \\
\hline Tall & $\begin{array}{l}\text { WAUBAY NWR } \\
\text { COMPLEX }\end{array}$ & $\begin{array}{c}\text { MARSHALL COUNTY } \\
\text { WPA }\end{array}$ & Buffalo Lake & 2010 & Rest & NA & Med & $2-4$ & $\{30-45, \mathrm{CO}\}$ & Burn \\
\hline Tall & $\begin{array}{l}\text { WAUBAY NWR } \\
\text { COMPLEX }\end{array}$ & $\begin{array}{c}\text { MARSHALL COUNTY } \\
\text { WPA }\end{array}$ & Buffalo Lake & 2011 & Rest & Rest & Low & $2-4$ & $\{0-30, \mathrm{CO}\}$ & $\begin{array}{l}\text { Graze within } \\
\text { window }\end{array}$ \\
\hline Tall & $\begin{array}{l}\text { WAUBAY NWR } \\
\text { COMPLEX }\end{array}$ & $\begin{array}{c}\text { MARSHALL COUNTY } \\
\text { WPA }\end{array}$ & Buffalo Lake & 2012 & Rest & Rest & Low & $2-4$ & $\{30-45, S B\}$ & $\begin{array}{l}\text { Burn within } \\
\text { window }\end{array}$ \\
\hline Tall & $\begin{array}{l}\text { WAUBAY NWR } \\
\text { COMPLEX }\end{array}$ & $\begin{array}{c}\text { MARSHALL COUNTY } \\
\text { WPA }\end{array}$ & Buss Paddock 1 & 2010 & Rest & NA & Low & $5+$ & $\{0-30, \mathrm{CO}\}$ & Burn/Graze \\
\hline Tall & $\begin{array}{l}\text { WAUBAY NWR } \\
\text { COMPLEX }\end{array}$ & $\begin{array}{c}\text { MARSHALL COUNTY } \\
\text { WPA }\end{array}$ & Buss Paddock 1 & 2011 & Graze & Defoliate & Low & 1 & $\{0-30, \mathrm{CO}\}$ & $\begin{array}{l}\text { Graze within } \\
\text { window }\end{array}$ \\
\hline Tall & $\begin{array}{l}\text { WAUBAY NWR } \\
\text { COMPLEX }\end{array}$ & $\begin{array}{c}\text { MARSHALL COUNTY } \\
\text { WPA }\end{array}$ & Buss Paddock 1 & 2012 & Graze & Defoliate & Low & 1 & $\{0-30, S B\}$ & $\begin{array}{l}\text { Graze within } \\
\text { window }\end{array}$ \\
\hline Tall & $\begin{array}{l}\text { WAUBAY NWR } \\
\text { COMPLEX }\end{array}$ & $\begin{array}{c}\text { MARSHALL COUNTY } \\
\text { WPA }\end{array}$ & Buss Paddock 2 & 2010 & Rest & NA & Low & $5+$ & $\{0-30, \mathrm{CO}\}$ & Burn/Graze \\
\hline Tall & $\begin{array}{l}\text { WAUBAY NWR } \\
\text { COMPLEX }\end{array}$ & $\begin{array}{c}\text { MARSHALL COUNTY } \\
\text { WPA }\end{array}$ & Buss Paddock 2 & 2011 & Graze & Defoliate & Low & 1 & $\{0-30, \mathrm{CO}\}$ & $\begin{array}{l}\text { Graze within } \\
\text { window }\end{array}$ \\
\hline Tall & $\begin{array}{l}\text { WAUBAY NWR } \\
\text { COMPLEX }\end{array}$ & $\begin{array}{c}\text { MARSHALL COUNTY } \\
\text { WPA }\end{array}$ & Buss Paddock 2 & 2012 & Graze & Defoliate & Low & 1 & $\{0-30, S B\}$ & $\begin{array}{l}\text { Graze within } \\
\text { window }\end{array}$ \\
\hline
\end{tabular}




\begin{tabular}{|c|c|c|c|c|c|c|c|c|c|c|}
\hline $\begin{array}{l}\text { Grass } \\
\text { Type }\end{array}$ & Complex & Org & Unit & $\begin{array}{c}\text { Management } \\
\text { Year }\end{array}$ & $\begin{array}{c}\text { Management } \\
\text { Applied }\end{array}$ & $\begin{array}{c}\text { Management } \\
\text { Classified }\end{array}$ & $\begin{array}{c}\text { Defoliation } \\
\text { Level }\end{array}$ & $\begin{array}{l}\text { Years Since } \\
\text { Defoliation }\end{array}$ & $\begin{array}{l}\text { Vegetation } \\
\text { State }\end{array}$ & $\begin{array}{c}\text { Management } \\
\text { Recommendation } \\
\text { Year } t+1\end{array}$ \\
\hline Tall & $\begin{array}{l}\text { WAUBAY NWR } \\
\text { COMPLEX }\end{array}$ & $\begin{array}{c}\text { MARSHALL COUNTY } \\
\text { WPA }\end{array}$ & Jensen East & 2009 & Graze & NA & High & 1 & $\{30-45, K B\}$ & NA \\
\hline Tall & $\begin{array}{l}\text { WAUBAY NWR } \\
\text { COMPLEX }\end{array}$ & $\begin{array}{c}\text { MARSHALL COUNTY } \\
\text { WPA }\end{array}$ & Jensen East & 2010 & Rest & NA & High & $2-4$ & $\{0-30, K B\}$ & Graze \\
\hline Tall & $\begin{array}{l}\text { WAUBAY NWR } \\
\text { COMPLEX }\end{array}$ & $\begin{array}{c}\text { MARSHALL COUNTY } \\
\text { WPA }\end{array}$ & Jensen East & 2011 & Graze & Defoliate & High & 1 & $\{30-45, K B\}$ & $\begin{array}{l}\text { Burn within } \\
\text { window }\end{array}$ \\
\hline Tall & $\begin{array}{l}\text { WAUBAY NWR } \\
\text { COMPLEX }\end{array}$ & $\begin{array}{c}\text { ROBERTS COUNTY } \\
\text { WPA }\end{array}$ & $\begin{array}{l}\text { Berward } \\
\text { Paddock } 4\end{array}$ & 2010 & Rest & NA & Low & $2-4$ & $\{30-45, \mathrm{~KB}\}$ & Graze \\
\hline Tall & $\begin{array}{l}\text { WAUBAY NWR } \\
\text { COMPLEX }\end{array}$ & $\begin{array}{c}\text { ROBERTS COUNTY } \\
\text { WPA }\end{array}$ & $\begin{array}{l}\text { Berward } \\
\text { Paddock } 4\end{array}$ & 2011 & Graze & Defoliate & Med & 1 & $\{30-45, K B\}$ & $\begin{array}{l}\text { Burn within } \\
\text { window }\end{array}$ \\
\hline Tall & $\begin{array}{l}\text { WAUBAY NWR } \\
\text { COMPLEX }\end{array}$ & $\begin{array}{c}\text { ROBERTS COUNTY } \\
\text { WPA }\end{array}$ & $\begin{array}{c}\text { Berward } \\
\text { Paddock } 4\end{array}$ & 2012 & Rest & Rest & Med & $2-4$ & $\{30-45, \mathrm{CO}\}$ & $\begin{array}{l}\text { Burn within } \\
\text { window }\end{array}$ \\
\hline Tall & $\begin{array}{l}\text { WAUBAY NWR } \\
\text { COMPLEX }\end{array}$ & $\begin{array}{c}\text { ROBERTS COUNTY } \\
\text { WPA }\end{array}$ & $\begin{array}{l}\text { Berward } \\
\text { Paddock } 5\end{array}$ & 2010 & Rest & NA & Low & $2-4$ & $\{30-45, \mathrm{CO}\}$ & Burn \\
\hline Tall & $\begin{array}{l}\text { WAUBAY NWR } \\
\text { COMPLEX }\end{array}$ & $\begin{array}{c}\text { ROBERTS COUNTY } \\
\text { WPA }\end{array}$ & $\begin{array}{c}\text { Berward } \\
\text { Paddock } 5\end{array}$ & 2011 & Rest & Rest & Low & $2-4$ & $\{30-45, \mathrm{CO}\}$ & $\begin{array}{l}\text { Burn within } \\
\text { window }\end{array}$ \\
\hline Tall & $\begin{array}{l}\text { WAUBAY NWR } \\
\text { COMPLEX }\end{array}$ & $\begin{array}{c}\text { ROBERTS COUNTY } \\
\text { WPA }\end{array}$ & $\begin{array}{c}\text { Berward } \\
\text { Paddock } 5\end{array}$ & 2012 & Rest & Rest & Low & $2-4$ & $\{0-30, \mathrm{CO}\}$ & $\begin{array}{c}\text { Graze within } \\
\text { window }\end{array}$ \\
\hline Tall & $\begin{array}{l}\text { WAUBAY NWR } \\
\text { COMPLEX }\end{array}$ & $\begin{array}{c}\text { ROBERTS COUNTY } \\
\text { WPA }\end{array}$ & Wike Paddock 1 & 2009 & Rest & NA & Low & $5+$ & $\{0-30, K B\}$ & NA \\
\hline Tall & $\begin{array}{l}\text { WAUBAY NWR } \\
\text { COMPLEX }\end{array}$ & $\begin{array}{c}\text { ROBERTS COUNTY } \\
\text { WPA }\end{array}$ & Wike Paddock 1 & 2010 & Rest & NA & Low & $5+$ & $\{0-30, K B\}$ & Burn/Graze \\
\hline Tall & $\begin{array}{l}\text { WAUBAY NWR } \\
\text { COMPLEX }\end{array}$ & $\begin{array}{c}\text { ROBERTS COUNTY } \\
\text { WPA }\end{array}$ & Wike Paddock 1 & 2011 & Burn & $\begin{array}{l}\text { Burn w/in } \\
\text { window }\end{array}$ & Low & 1 & $\{0-30, K B\}$ & $\begin{array}{l}\text { Burn within } \\
\text { window }\end{array}$ \\
\hline Tall & $\begin{array}{l}\text { WAUBAY NWR } \\
\text { COMPLEX }\end{array}$ & $\begin{array}{c}\text { ROBERTS COUNTY } \\
\text { WPA }\end{array}$ & Wike Paddock 1 & 2012 & Burn & Defoliate & Low & 1 & $\{30-45, K B\}$ & $\begin{array}{l}\text { Burn within } \\
\text { window }\end{array}$ \\
\hline Tall & $\begin{array}{l}\text { WAUBAY NWR } \\
\text { COMPLEX }\end{array}$ & $\begin{array}{c}\text { ROBERTS COUNTY } \\
\text { WPA }\end{array}$ & Wike Paddock 2 & 2009 & Rest & NA & Low & $5+$ & $\{0-30, \mathrm{CO}\}$ & NA \\
\hline Tall & $\begin{array}{l}\text { WAUBAY NWR } \\
\text { COMPLEX }\end{array}$ & $\begin{array}{c}\text { ROBERTS COUNTY } \\
\text { WPA }\end{array}$ & Wike Paddock 2 & 2010 & Rest & NA & Low & $5+$ & $\{0-30, \mathrm{CO}\}$ & Burn/Graze \\
\hline Tall & $\begin{array}{l}\text { WAUBAY NWR } \\
\text { COMPLEX }\end{array}$ & $\begin{array}{c}\text { ROBERTS COUNTY } \\
\text { WPA }\end{array}$ & Wike Paddock 2 & 2011 & Burn & $\begin{array}{l}\text { Burn w/in } \\
\text { window }\end{array}$ & Low & 1 & $\{0-30, \mathrm{CO}\}$ & $\begin{array}{l}\text { Graze within } \\
\text { window }\end{array}$ \\
\hline
\end{tabular}




\begin{tabular}{|c|c|c|c|c|c|c|c|c|c|c|}
\hline $\begin{array}{l}\text { Grass } \\
\text { Type }\end{array}$ & Complex & Org & Unit & $\begin{array}{c}\text { Management } \\
\text { Year }\end{array}$ & $\begin{array}{c}\text { Management } \\
\text { Applied }\end{array}$ & $\begin{array}{l}\text { Management } \\
\text { Classified }\end{array}$ & $\begin{array}{c}\text { Defoliation } \\
\text { Level }\end{array}$ & $\begin{array}{l}\text { Years Since } \\
\text { Defoliation }\end{array}$ & $\begin{array}{l}\text { Vegetation } \\
\text { State }\end{array}$ & $\begin{array}{c}\text { Management } \\
\text { Recommendation } \\
\text { Year } t+1\end{array}$ \\
\hline Tall & $\begin{array}{l}\text { WAUBAY NWR } \\
\text { COMPLEX }\end{array}$ & $\begin{array}{c}\text { ROBERTS COUNTY } \\
\text { WPA }\end{array}$ & Wike Paddock 2 & 2012 & Rest & Rest & Low & $2-4$ & $\{0-30, \mathrm{CO}\}$ & $\begin{array}{c}\text { Graze within } \\
\text { window }\end{array}$ \\
\hline Tall & WINDOM WMD & $\begin{array}{l}\text { COTTONWOOD } \\
\text { COUNTY WPA }\end{array}$ & $\begin{array}{c}\text { Des Moines } \\
\text { River WPA } \\
\text { NORTH }\end{array}$ & 2010 & Burn & NA & Med & 1 & $\{60-100, S B\}$ & Graze \\
\hline Tall & WINDOM WMD & $\begin{array}{l}\text { COTTONWOOD } \\
\text { COUNTY WPA }\end{array}$ & $\begin{array}{l}\text { Des Moines } \\
\text { River WPA } \\
\text { NORTH }\end{array}$ & 2011 & Rest & Rest & Low & $2-4$ & $\{60-100, S B\}$ & $\begin{array}{l}\text { Burn within } \\
\text { window }\end{array}$ \\
\hline Tall & WINDOM WMD & $\begin{array}{l}\text { COTTONWOOD } \\
\text { COUNTY WPA }\end{array}$ & $\begin{array}{c}\text { Des Moines } \\
\text { River WPA } \\
\text { NORTH }\end{array}$ & 2012 & Burn & $\begin{array}{l}\text { Burn w/in } \\
\text { window }\end{array}$ & Med & 1 & $\{60-100, S B\}$ & $\begin{array}{l}\text { Burn within } \\
\text { window }\end{array}$ \\
\hline Tall & WINDOM WMD & $\begin{array}{l}\text { COTTONWOOD } \\
\text { COUNTY WPA }\end{array}$ & $\begin{array}{l}\text { Des Moines } \\
\text { River WPA } \\
\text { SOUTH }\end{array}$ & 2010 & Rest & NA & Low & $2-4$ & $\{45-60, \mathrm{CO}\}$ & Burn \\
\hline Tall & WINDOM WMD & $\begin{array}{l}\text { COTTONWOOD } \\
\text { COUNTY WPA }\end{array}$ & $\begin{array}{l}\text { Des Moines } \\
\text { River WPA } \\
\text { SOUTH }\end{array}$ & 2011 & Burn & $\begin{array}{l}\text { Burn w/in } \\
\text { window }\end{array}$ & Low & 1 & $\{60-100, S B\}$ & $\begin{array}{l}\text { Burn within } \\
\text { window }\end{array}$ \\
\hline Tall & WINDOM WMD & $\begin{array}{l}\text { COTTONWOOD } \\
\text { COUNTY WPA }\end{array}$ & $\begin{array}{l}\text { Des Moines } \\
\text { River WPA } \\
\text { SOUTH }\end{array}$ & 2012 & Burn & $\begin{array}{l}\text { Burn w/in } \\
\text { window }\end{array}$ & Med & 1 & $\{60-100, S B\}$ & $\begin{array}{l}\text { Burn within } \\
\text { window }\end{array}$ \\
\hline
\end{tabular}

\section{Field Descriptions}

Management Year: The management year runs from September 1 of the previous calendar year to August 31 of the current calendar year.

For example:

Management Year 2009: September 1, 2008-August 31, 2009

Management Year 2010: September 1, 2009-August 31, 2010

Management Year 2011: September 1, 2010-August 31, 2011

Management Year 2012: September 1, 2011-August 31, 2012

This can be generically written as follows:

Management Year $t$ : September 1, $t$-1-August 31, $t$ 
Management Applied: The management action that was applied during the management year. This is taken directly from what the cooperator entered into the database as the combination of all management records for a given unit within the management year.

Management Classified: Classification of the applied management into one of the four recognized alternative Native Prairie Adaptive Management (NPAM) actions tallgrass units (rest, graze within window, burn within window, defoliate). These classifications are made according to defined NPAM rules. For tallgrass units, with the exception of units that received a rest, 'Management Classified' will be different from 'Management Applied'. This is due to classification of the timing of the action relative to the cool-season window. Some combinations of actions on a single unit within the same management year do not fall within the existing rules and are therefore classified as 'Unclassifiable'. If you have questions about the classification of the management on your unit, please contact the NPAM Project Coordinator for an explanation specific to your situation. 'Management Classified' for tallgrass units in 2009 and 2010 is listed as NA. We did not need to classify the 2009 management because, lacking monitoring data for 2008, we could not use the 2009 management to update model weights. Additionally, modification of the tallgrass framework in 2010 (specifically, the addition of the cool-season window aspect) made it such that we did not have the necessary data (specifically, the phenological data) to classify the applied actions according to their timing relative to the cool-season window.

Defoliation Level: A seven-year window was used to classify the management history of each unit into one of three defoliation categories: Low, Med, or High.

Seven-year windows for each management year:

2009: From 2003 to 2009

2010: From 2004 to 2010

2011: From 2005 to 2011

2012: From 2006 to 2012

This can be generically written as follows:

$t$ : From $(t-6)$ to $t$

Year $t$, the most recent management year, is included as the closing year of the seven-year window.

Assignment into categories was based on a combination of the years since the last defoliation action (graze, burn, burn/graze, or hay) was applied on the unit, and the total number of defoliations (graze, burn, burn/graze, or hay) that were carried out during the entire seven-year window (maximum of one defoliation per management year; that is, a unit was either defoliated or not defoliated in a given year).

Years Since Defoliation: Classifies each unit into one of three 'Years Since Defoliation' levels: 1, 2-4, or 5+.

These classifications denote the number of years it has been since the unit was last defoliated (graze, burn, burn/graze, or hay). A seven-year window was used to determine this state. A ' 1 ' means the unit was defoliated during the most recent management year. 
Vegetation State: Vegetation state is defined by the proportions of the four main vegetative components: NP, SB, KB, and RM. $\mathrm{NP}=$ Proportion of the unit that consists of native grasses and forbs (NP). Based on an average of all transects on the unit with monitoring data for the given management year.

$\mathrm{SB}=$ Proportion of the unit that consists of the cool-season invasive species smooth brome (SB). Based on an average of all transects on the unit with monitoring data for the given management year.

$\mathrm{KB}=$ Proportion of the unit that consists of the cool-season invasive species Kentucky bluegrass (KB). Based on an average of all transects on the unit with monitoring data for the given management year.

$\mathrm{RM}=$ Proportion of the unit that consists of vegetation other than NP, SB, or KB. Based on an average of all transects on the unit with monitoring data for the given management year. $\mathrm{RM}=$ remainder.

The Vegetation state is shown as $\{x, y\}$, where: $x=\mathrm{NP}$ level and $y=$ dominant invader (that is, $\mathrm{SB}, \mathrm{KB}, \mathrm{CO}$, or RM).

NP level: The four NP levels are: 0-30 percent, 30-45 percent, $45-60$ percent, $60-100$ percent. A unit is classified into one of these four NP levels based on the NP proportion.

Dominant Invader: The dominant invader was determined as follows:

$\mathrm{RM}$ is dominant if: $\mathrm{RM} /(\mathrm{SB}+\mathrm{KB}+\mathrm{RM})>=0.67$

Otherwise,

SB is dominant if: $\mathrm{SB} /(\mathrm{SB}+\mathrm{KB})>=0.67$

$\mathrm{KB}$ is dominant if: $\mathrm{KB} /(\mathrm{SB}+\mathrm{KB})>=0.67$

If neither $\mathrm{SB}$ nor $\mathrm{KB}$ is dominant, it is considered a state of co-dominance $(\mathrm{CO})$ by $\mathrm{SB}$ and $\mathrm{KB}$.

Note: Vegetation proportions are calculated excluding the following plant codes: -9 (no data), 24 and 47 (open water, SD and ND list respectively), 91 (barren), and 92 and 99 (other, SD and ND list respectively).

Management Recommendation Year $\boldsymbol{t}+\mathbf{1}$ : Recommended management action for the upcoming management year. The recommendation is based on unit-specific defoliation level, years since defoliation, and vegetation state for the current management year, as well as on the current knowledge state and decision policy for the management year (see appendix 2 for the 2010 policy, and figure 14 and figure 15 in the main text for the 2011 and 2012 policies, respectively). 